ERNEST $\square R L A N D \square L A W R E N C E$

BERKELEY NATIDNAL LABDRATIRY

(Ii) (A) 2$)$

Two-Dimensional NMR Investigations

of the Dynamic Conformations of

Phospholipids and Liquid Crystals

Mei Hong

Materials Sciences Division

RECEIVED

May 1996

Ph.D. Thesis

MAR 181999

OSTI 


\section{DISCLAIMER}

This document was prepared as an account of work sponsored by the United States Government. While this document is believed to contain correct information, neither the United States Government nor any agency thereof, nor The Regents of the University of California, nor any of their employees, makes any warranty, express or implied, or assumes any legal responsibility for the accuracy, completeness, or usefulness of any information, apparatus, product, or process disclosed, or represents that its use would not infringe privately owned rights. Reference herein to any specific commercial product, process, or service by its trade name, trademark, manufacturer, or otherwise, does not necessarily constitute or imply its endorsement, recommendation, or favoring by the United States Government or any agency thereof, or The Regents of the University of California. The views and opinions of authors expressed herein do not necessarily state or reflect those of the United States Government or any agency thereof, or The Regents of the University of California.

Ernest Orlando Lawrence Berkeley National Laboratory is an equal opportunity employer. 


\section{DISCLAIMER}

\section{Portions of this document may be illegible in electronic image products. Images are produced from the best available original document.}




\title{
Two-Dimensional NMR Investigations of the Dynamic Conformations of Phospholipids and Liquid Crystals
}

\author{
Mei Hong \\ Ph.D. Thesis \\ Department of Chemistry \\ University of California, Berkeley \\ and \\ Materials Sciences Division \\ Ernest Orlando Lawrence Berkeley National Laboratory \\ University of California \\ Berkeley, CA 94720
}

May 1996 


\section{Two-Dimensional NMR Investigations of the Dynamic Conformations of Phospholipids and Liquid Crystals \\ by \\ Mei Hong}

B.A. (Mount Holyoke College) 1992

A dissertation submitted in partial satisfaction of the requirements for the degree of

Doctor of Philosophy

in

Chemistry

in the

GRADUATE DIVISION

of the

UNIVERSITY OF CALIFORNIA, BERKELEY

Committee in charge:

Professor Alexander Pines, Chair

Professor Paul Alivisatos

Professor Jeffrey A. Reimer 


\section{Two-Dimensional NMR Investigations of the Dynamic Conformations of Phospholipids and Liquid Crystals}

Copyright ㄷ 1996

by

Mei Hong

The U.S. Department of Energy has the right to use this document for any purpose whatsoever including the right to reproduce all or any part thereof 


\author{
Abstract \\ Two-Dimensional NMR Investigations of \\ the Dynamic Conformations of Phospholipids and Liquid Crystals \\ by \\ Mei Hong \\ Doctor of Philosophy in Chemistry \\ University of California, Berkeley \\ Professor Alexander Pines, Chair
}

Two-dimensional ${ }^{13} \mathrm{C},{ }^{1} \mathrm{H}$ and ${ }^{31} \mathrm{P}$ nuclear magnetic resonance (NMR) techniques are developed and used to study molecular structure and dynamics in liquid-crystalline systems, primarily phospholipids and nematic liquid crystals. NMR spectroscopy characterizes molecular conformation in terms of orientations and distances of molecular segments. In anisotropically mobile systems, this is achieved by measuring motionally-averaged nuclear dipolar couplings and chemical shift anisotropies. The short-range couplings yield useful bond order parameters, while the long-range interactions constrain the overall conformation. In this work, techniques for probing proton dipolar local fields are further developed to obtain highlyresolved dipolar couplings between protons and rare spins. By exploiting variable-angle sample spinning techniques, orientation-sensitive NMR spectra are resolved according to sitespecific isotropic chemical shifts. Moreover, the signs and magnitudes of various short-range dipolar couplings are obtained. They are used in novel theoretical analyses that provide information about segmental orientations and their distributions. Such information is obtained in a model-independent fashion or with physically reasonable assumptions.

The structural investigation of phospholipids is focused on the dynamic conformations of the headgroup and glycerol backbone of liquid-crystalline phosphocholine. Several structural features are identified, including the headgroup bend from the glycerol backbone, the 
relative stiffness of the glycerol and its adjacent segments, the proximity of the headgroup $\alpha$ segment to the $s n-2$ chain carboxyl group, and the estimated relative populations of various headgroup conformations. Constrained by the dipolar couplings and chemical shift anisotropies measured here and NMR data from the literature, a new single-conformation model is proposed for the core of the phosphocholine molecule.

In the nematic liquid crystal 4-pentyl-4'-biphenylcarbonitrile, both short- and longrange dipolar couplings between carbons and protons are obtained. The directly-bonded dipolar couplings allow bond order parameters to be calculated for the aromatic core of the molecule, thus providing information on the conformational order of the molecule. For the first time, long-range dipolar couplings are resolved and assigned, yielding a large number of new constraints for structural modeling of this system. 
To Klaus 


\section{TABLE OF CONTENTS}

CHAPTER 1

INTRODUCTION 1

\section{CHAPTER 2}

THEORY OF SOLID-STATE NMR 3

2.1 Nuclear Spin Interactions............................................ 3

2.2 Semiclassical Description of NMR - Magnetization.......................... 6

2.3 Quantum Mechanical Description of NMR - Density Operator ............ 9

2.4 Two-Dimensional Fourier NMR ............................................. 14

2.5 Powder Lineshapes - Orientation Dependence in Solid-State NMR ....... 21

2.6 Sample Spinning - Averaging in Physical Space........................... 26

2.7 Average Hamiltonian Theory - Averaging in Spin Space ................. 29

2.8 Proton-Detected Local Field Spectroscopy ................................ 36

\section{CHAPTER 3}

PROPERTIES OF LIQUID-CRYSTALLINE PHOSPHOLIPIDS...............43

3.1 Biological Importance............................................ 43

3.2 Chemical Structure ................................................ 45

3.3 Organized Assemblies................................................... 48

3.4 Physical Techniques for Studying Phospholipids .......................51

3.5 Computer Simulations of Phospholipid Conformation and

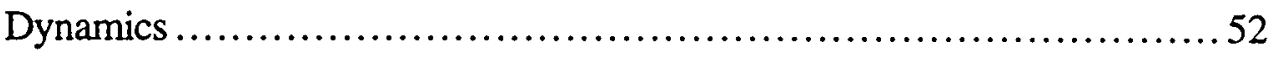

3.6 NMR Techniques for Studying Phospholipids ..........................5 53

${ }^{2} \mathrm{H}$ Quadrupolar Couplings................................................5 53

${ }^{31} \mathrm{P}$ Chemical Shifts ................................................... 54

${ }^{13} \mathrm{C}$ Chemical Shifts.................................................... 55

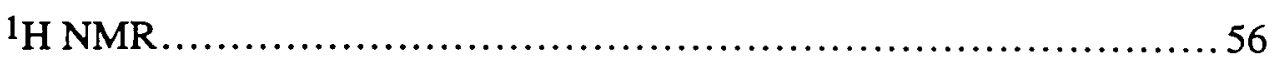

\section{CHAPTER 4}

THEORY OF ORDER TENSOR FOR PHOSPHOLIPIDS AND

LIQUID CRYSTALS ........................................................ 60

4.1 Effects of Liquid Crystallinity on Phospholipid NMR Spectra .............60

4.2 Derivation of Order Tensor................................................ 63 
4.3 Phenomenological Description of Order Tensor ........................68

4.4 Relation Between Order Tensor and Order Parameter............................69

4.5 Strategies for Determining Order Tensors ................................ 72

4.6 Orientation Distributions from Order Tensors.................................... 74

4.7 Probability Limits from Bond Order Parameters................................. 80

Model-Independent Probabilities from Negative Order Parameters ........ 80

Probability Limits under the Assumption of Broadening ................... 84

Probability Limits from Positive Order Parameters.............................. 86

Model-Independent Probabilities from Chemical Shift Anisotropies........89

\section{CHAPTER 5}

NMR MEASUREMENTS OF PHOSPHOCHOLINE LIPIDS............... 91

5.1 General Experimental Conditions................................................91

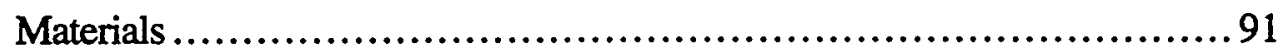

Sample Preparation ................................................. 92

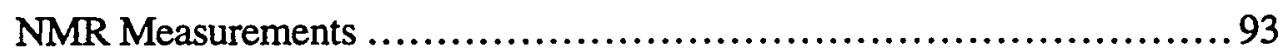

5.2 Short-Range ${ }^{13} \mathrm{C}-1 \mathrm{H}$ Dipolar Couplings ................................ 95

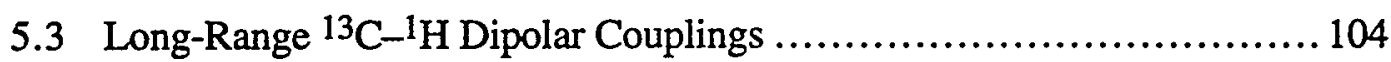

$5.4{ }^{31} \mathrm{P}-1 \mathrm{H}$ Dipolar Couplings ............................................. 111

$5.5{ }^{1} \mathrm{H}-1 \mathrm{H}$ Dipolar Couplings ........................................... 116

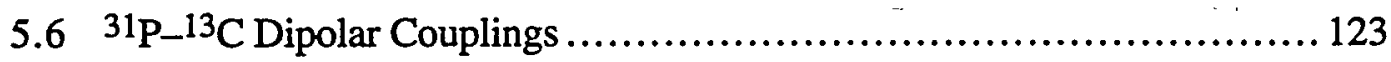

$5.7{ }^{13} \mathrm{C}-{ }^{13} \mathrm{C}$ Dipolar Couplings.......................................... 125

5.8 Determination of Dipolar Coupling Signs by DISTINCT NMR............ 129

DISTINCT with Proton-Detected-Local-Field Evolution.................. 130

Effects of Powder Average........................................... 133

DISTINCT with Carbon-Detected-Local-Field Evolution ................. 140

$5.9{ }^{13} \mathrm{C}$ Chemical Shift Anisotropies ................................... 143

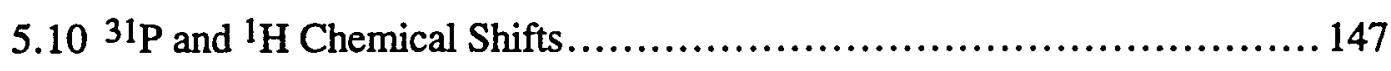

5.11 Summary of NMR Order Parameters in Lecithin ....................... 152

\section{CHAPTER 6}

PHOSPHOCHOLINE STRUCTURE IN THE HEADGROUP AND GLYCEROL BACKBONE.................................................... 160

6.1 Acyl Chain Order and Mobility ..................................... 160

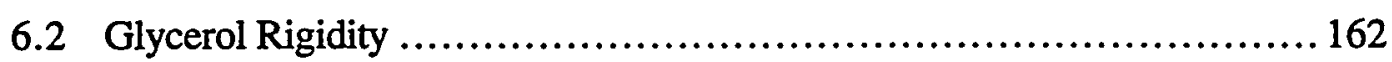

6.3 Rotational Axes Orientations ........................................ 163 
6.4 Headgroup and Acyl Chain Bends ................................... 167

6.5 Enantiomeric Headgroup Conformations............................... 169

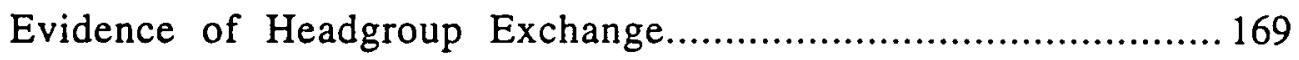

Populations of Headgroup Conformers...................................... 172

General Requirements for $\mathrm{C}-\mathrm{H}$ Degeneracies in Liquid-Crystalline

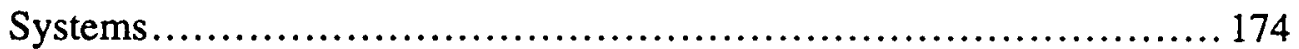

6.6 Single-Conformation Model for Phosphocholine Core .................... 176

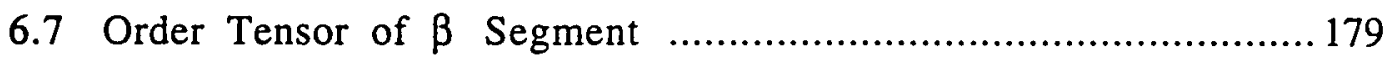

\section{CHAPTER 7}

\section{DETFRMINATION OF C-H DIPOLAR COUPLINGS IN LIQUID}

\section{CRYSTALS}

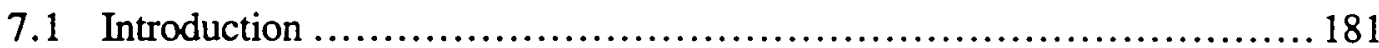

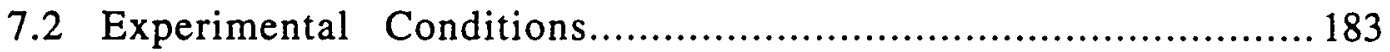

7.3 Measurement of C-H Dipolar Couplings............................. 188

7.4 Qualitative Assignment of Long-Range C-H Couplings ................. 195

7.5 Quantitative Assignment of Long-Range C-H Couplings.................. 198

7.6 Order Parameters from Short-Range C-H Couplings........................... 204

7.7 Experimental Precision and Accuracy .............................. 207

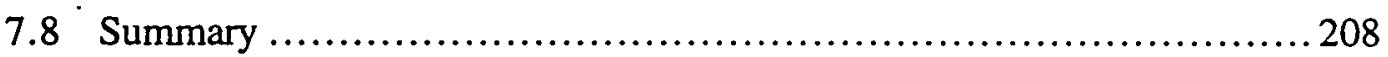

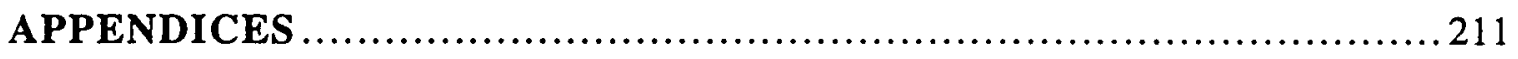

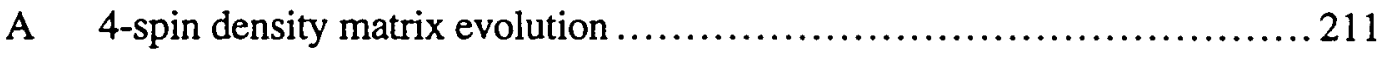

B Equivalence of Motional Averaging in Fast Motional Limit ................2 213

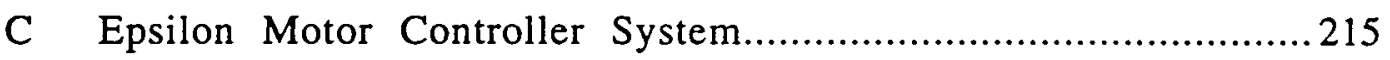

D Computer Programs................................................ 217

Model-Independent Orientation Probabilities from a Negative Order

Parameter ........................................................... 218

Orientation Distributions from Segmental Order Tensors...................... 222 


\section{LIST OF FIGURES}

Figure 2.1 Larmor precession of a magnetic moment in a magnetic field............... 8

Figure 2.2 NMR powder spectra of polycrystalline solids ........................26

Figure 2.3 (a) PDLF and (b) SLF pulse sequences ............................. 38

Figure 2.4 Schematic (a) PDLF and (b) SLF stick spectra .......................... 39

Figure 3.1 Three main types of membrane structural lipids .......................46

Figure 3.2 Organized phospholipid assemblies................................55

Figure 4.1 $\quad \mathrm{L}_{\alpha}$-lecithin ${ }^{31} \mathrm{P}$ single-pulse chemical shift spectrum ..................60

Figure 4.2 Schematic of phospholipid bilayers ..............................6 61

Figure 4.3 $\mathrm{L}_{\alpha}$-lecithin ${ }^{31} \mathrm{P}$ cross-polarized chemical shift spectrum ................62

Figure 4.4 Dipolar spectra of a rigid solid and an anisotropically-mobile lipid ......62

Figure 4.5 Director distributions in a segment-fixed frame.............................. 77

Figure 4.6 Relation of principal order parameters with shape parameters of a biaxial Gaussian distribution......................................... 79

Figure 4.7 Orientation distributions and probabilities constrained by bond order

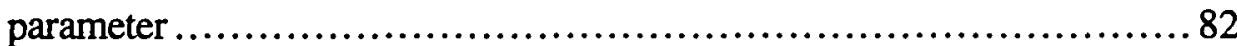

Figure 4.8 Model-independent upper-limit probabilities for a negative order parameter ....................................................... 83

Figure 4.9 Upper-limit probabilities parameterized by bond order parameter........84

Figure 4.10 Upper-limit probabilities for broadened distributions................... 86

Figure 4.11 Upper-limit probabilities parameterized by minimum Gaussian

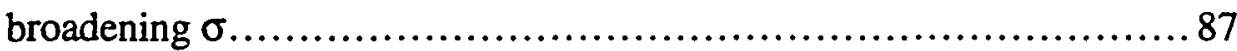

Figure 4.12 Upper-limit probabilities for a positive order parameter ................. 88

Figure 5.1 PDLF-SAS pulse sequence for measuring $\mathrm{C}-\mathrm{H}$ dipolar couplings ...... 95

Figure 5.2 $\mathrm{L}_{\alpha}$-lecithin C-H PDLF spectra obtained under SAS........................ 96

Figure 5.3 Analysis of the signs and magnitudes of dipolar couplings .............. 98

Figure 5.4 Headgroup and glycerol regions of $\mathrm{L}_{\alpha}$-DMPC C-H PDLF spectra obtained under OMAS. ................................................... 101

Figure 5.5 Double bond region of $\mathrm{L}_{\alpha}$-lecithin C-H PDLF spectrum obtained under SAS............................................................ 102

Figure 5.6 Averaged C-H dipolar couplings and the corresponding order

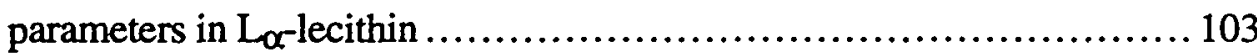

Figure $5.7 \quad{ }^{13} \mathrm{C} /{ }^{1} \mathrm{H}$ HETCOR pulse sequences...................................... 105

Figure $5.8 \quad{ }^{13} \mathrm{CO}$-DMPC HETCOR spectrum obtained without proton $\mathrm{T}_{2}$ filter $\ldots 107$ 
Figure 5.9 ${ }^{13} \mathrm{CO} 2$ cross sections from DMPC HETCOR spectra ................... 108

Figure 5.10 DMPC ${ }^{13} \mathrm{C}$ CPMAS spectra with ${ }^{1} \mathrm{H} \mathrm{T}_{2}$ filter of varying duration $\tau$...... 110

Figure 5.11 $\mathrm{P}-\mathrm{H} /{ }^{\beta 1} \mathrm{P}$ CSA $2 \mathrm{D}$ correlation spectra of three $\mathrm{L}_{\alpha}$-phospholipids ........ 113

Figure 5.12 Pulse sequence correlating $\mathrm{P}-\mathrm{H}$ and $\mathrm{H}-\mathrm{H}$ couplings ................... 114

Figure 5.13 $\mathrm{L}_{\alpha}$-lecithin $\mathrm{P}-\mathrm{H} / \mathrm{H}-\mathrm{H}$ correlation spectrum.......................... 115

Figure 5.14 $\mathrm{L}_{\alpha}$-lecithin $\mathrm{H}-\mathrm{H} /{ }^{1} \mathrm{H}$ CSA correlation spectrum ....................... 117

Figure 5.15 $L_{\alpha}$-lecithin ${ }^{13} \mathrm{C} /{ }^{1} \mathrm{H}$ MAS chemical shift correlation spectrum ............ 118

Figure 5.16 ${ }^{1} \mathrm{H}$ WISE pulse sequence under SAS................................. 119

Figure $5.17 \quad L_{\alpha}$-lecithin ${ }^{1} \mathrm{H}$ WISE spectrum obtained under SAS ................... 121

Figure 5.18 $\mathrm{L}_{\alpha}$-DMPC ${ }^{13} \mathrm{C}$ WISE spectrum obtained under OMAS................. 122

Figure $5.19 \quad L_{\alpha}$-lecithin $\mathrm{P}-\mathrm{C}$ dipolar couplings .................................. 124

Figure $5.20 \quad{ }^{13} \mathrm{C}$ SLF pulse sequence under SAS................................ 125

Figure 5.21 13CO2-DMPC SLF spectrum obtained under SAS with dipolar

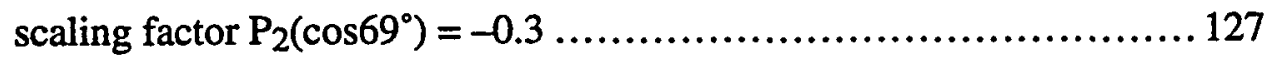

Figure 5.22 ${ }^{13} \mathrm{CO}-\mathrm{DMPC}$ SLF spectrum obtained under SAS with dipolar

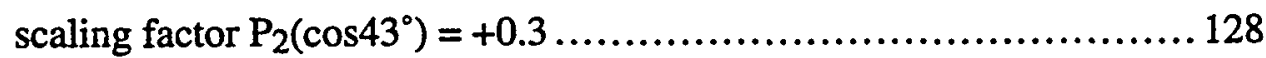

Figure 5.23 DISTINCT pulse sequence................................................. 131

Figure $5.24 \quad$ Simulated dipolar spectra........................................... 134

Figure 5.25 Calculated antiphase dipolar-J spectra as a function of $P_{2}(\cos \theta) \delta_{d}$

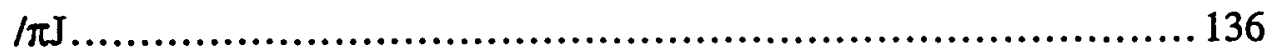

Figure 5.26 $\mathrm{L}_{\alpha}$-lecithin 2D DISTINCT spectrum.........................................137

Figure 5.27 $\mathrm{L}_{\alpha}$-lecithin 1D DISTINCT spectra beginning with ${ }^{1} \mathrm{H}$ magnetization .... 140

Figure 5.28 $\mathrm{L}_{\alpha}$-lecithin 1D DISTINCT spectrum beginning with ${ }^{13} \mathrm{C}$ magnetization ................................................... 142

Figure 5.29 SAS pulse sequence for measuring ${ }^{13} \mathrm{C}$ chemical shift anisotropies ...... 143

Figure $5.30 \quad \mathrm{~L}_{\alpha}$-lecithin $2 \mathrm{D}{ }^{13} \mathrm{C}$ SAS spectrum ............................... 145

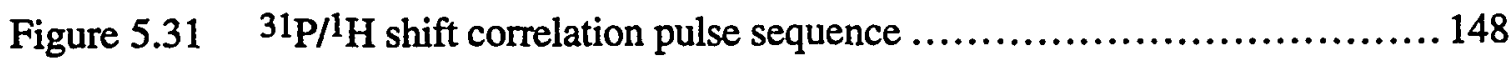

Figure 5.32 Lecithin ${ }^{31} \mathrm{P} / 1 \mathrm{H}$ CSA correlation 2D spectrum ........................ 149

Figure 5.33 ${ }^{1} \mathrm{H}$ chemical shift cross sections correlated with the $90^{\circ}$ edge of the $31 \mathrm{P}$ powder patterns.......................................................... 150

Figure 5.34 Lo-lecithin 2D ${ }^{1} \mathrm{H}$ exchange spectrum................................ 151

Figure 5.35 NMR order parameters in $\mathrm{L}_{\alpha}$-phosphocholine ....................... 156

Figure 6.1 Two DMPC conformations in a crystal unit cell......................... 171

Figure 6.2 A new structure proposed for the core of $L_{\alpha}$-phosphocholine.............. 178

Figure 7.1 (a) HETCOR and (b) reduced-3D pulse sequences .................... 186 
Figure 7.2 4-pentyl-4'-biphenylcarbonitrile (5CB),.................................. 188

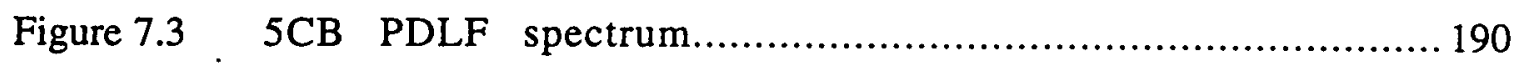

Figure 7.4 Directly-bonded C-H dipolar couplings in 5CB......................... 193

Figure 7.5 $5 \mathrm{CB}{ }^{13} \mathrm{C} / 1 \mathrm{H}$ chemical shift correlation spectrum....................... 197

Figure 7.6 5CB reduced-3D spectrum with ${ }^{1} \mathrm{H}$ PDLF:CS dwell ratio of $4 \ldots \ldots \ldots \ldots . .200$

Figure 7.7 5CB reduced-3D spectrum with ${ }^{1} \mathrm{H}$ PDLF:CS dwell ratio of 2........... 202

Figure B.1 Equivalence of motional averaging of a phenyl ring ..................213 


\section{LIST OF TABLES}

Table 2.1 Fourier transforms of some common functions ...................... 15

Table 3.1 Some naturally occurring fatty acids .............................45

Table 3.2 Headgroups of glycerophospholipids and sphingolipids................47

Table 5.1 NMR ${ }^{13} \mathrm{C}$ chemical shift order parameters in $\mathrm{L}_{\alpha}$-lecithin .............. 154

Table 5.2 Several NMR dipolar order parameters and ${ }^{31} \mathrm{P}$ chemical shift order parameter in phosphocholine........................................... 155

Table 6.1 Torsion angles in the headgroup and glycerol backbone of singlecrystal phosphocholine ........................................... 170

Table 7.1 Phase cycles for the reduced-3D experiment......................... 187

Table 7.2 Directly-bonded C-H dipolar couplings in 5CB........................ 195

Table 7.3 Resolved and assigned long-range $\mathrm{C}-\mathrm{H}$ dipolar couplings in 5CB ......203

Table $7.4 \quad \mathrm{C}-\mathrm{H}$ order parameters of the $5 \mathrm{CB}$ phenylene rings ......................206 


\section{ACKNOWLEDGEMENTS}

In my four years of graduate research in Berkeley, I have benefited greatly from many people whom I had the fortune of working with. First of all, I would like to thank my thesis advisor, Alex Pines, for providing a dynamic and rich environment for research and the pursuit of my scientific interests. I am grateful to many postdocs and graduate students who have been a constant source of knowledge and discussions: Andrew Kolbert (DSM polymers) for his impressive NMR repertoire and for being a fun person; Russell Larsen (lowa State), for his many patient assistance in repairing equipment; Young Lee (Univ. Stockholm) for introducing me to basic experimental NMR; Dani Nanz (Univ. Zürich) for showing me the ins and outs of the ancient Bruker AM400 and for being a wonderful colleague; Susan De Paul for sharing many memorable graduate school experiences with me; Jay Shore (South Dakota), for helps in experiments and for asking many a time the ultimate question about life to keep me from losing the big picture; Shuanhu Wang for his welcome companionship.

I should not neglect to mention my friends and families outside the microcosm of the Pines group at the Berkeley chemistry department. Among them are especially Yirong Hu, Gerd Kocherdoeffer, Nazli Nomanbhoy, the Lichtensteins, and Emily Zedlar. Their companionship, understanding, humor and generosity brightened my life outside the graduate school. I am also grateful to my parents and my sister for their continual trust, encouragement, and spiritual support through my graduate school.

The most important person in my graduate school life is unquestionably Klaus Schmidt-Rohr (UMass). I am indebted to him for teaching me all about two-dimensional NMR, and for his ideas and many stimulating discussions about my research. Last but not the least, it is with great appreciation that I thank him for returning me a husband when I lost a postdoc. 


\section{CHAPTER 1 \\ INTRODUCTION}

This thesis presents the investigation of the molecular conformation of liquidcrystalline phospholipids and nematic liquid crystals by two-dimensional nuclear magnetic resonance (NMR) techniques. Both systems are in the liquid-crystalline state, which is intermediate between the ordered crystalline solid state and the isotropic and highly mobile liquid state. The amphiphilic phospholipids are the major structural component of biological membranes. They provide a fluid environment in which other membrane molecules such as proteins function, and perform mechanical and chemical functions important for various biological processes in living organisms. Structural details of lipid molecules are functionally important, as the contents of various lipid species in specific cell types are tightly controlled and deviations from the norm can lead to serious diseases. As to the synthetic liquid crystals, their importance is reflected in their wide-spread industrial application in displays. In order to understand the functions and to achieve rational control of the properties of these biological and synthetic liquid-crystalline materials, it is important to determine the molecular structure and dynamics in detail.

NMR spectroscopy is a powerful and effective probe of the structure and dynamics of liquid-crystalline materials. It is sensitive to structural features on the atomic length scale and to motions on a wide range of time scales. It is well suited for probing complex structures, especially for correlating different structural elements by means of multidimensional techniques. Most importantly, the combination of modern liquid-state and solid-state NMR methodologies is perhaps the most promising approach for investigating the dynamic conformations of molecules that undergo large-amplitude anisotropic motions. These molecules not only include the liquid-crystalline phospholipids and nematic liquid crystals, but also peptides and proteins in fluid membrane bilayers. 
The liquid-crystalline nature of the systems under investigation and the significant molecular weights of phospholipids $(\sim 700 \mathrm{~g} / \mathrm{mol})$ call for the combined use of various NMR techniques. The combination of ${ }^{13} \mathrm{C},{ }^{1} \mathrm{H}$, and ${ }^{31} \mathrm{P}$ NMR allows many nuclear spin interactions to be studied, in order to characterize various aspects of the molecular conformation. In the experiments performed, sample spinning constitutes an essential ingredient, as it affords the flexibility of scaling the nuclear spin interactions and resolving chemically-inequivalent sites. Magic-angle spinning, off-magic-angle spinning, switchingangle spinning, and the static (non-spinning) conditions are employed as appropriate for the various experiments. Advanced radiofrequency techniques such as homonuclear multiple-pulse decoupling are used, and new possibilities for transferring heteronuclear coherence are explored.

The molecular motions in liquid crystals impart conformational information to NMR spectra which would only yield bond lengths in powder isotropic solids. On the other hand, they also render interpretation of the NMR spectra complex. A special theory, the order tensor formalism, is necessary for extracting conformational information from the experimental NMR spectra in terms of segmental orientation probabilities.

This thesis is organized as follows. First, NMR theory and techniques pertinent to this work are described (chapter 2). The biological importance, chemical structure, and mesophase properties of phospholipids are then summarized. A brief survey of the research on phospholipid structures is also given (chapter 3). This is followed by a detailed description of the order tensor formalism and a novel model-free interpretation of bond order parameters (chapter 4). Subsequently, various one- and two-dimensional NMR measurements on phosphocholine lipids are presented and the data put into perspective (chapter 5). The structural implications of these results are discussed, and a NMR-derived conformation of the core of the phosphocholine molecule is presented (chapter 6). In chapter 7, the measurement of dipolar couplings in a nematic liquid crystal using novel 2D NMR techniques is described. 


\section{CHAPTER 2 \\ THEORY OF SOLID-STATE NMR}

\subsection{Nuclear Spin Interactions}

The predominant interaction experienced by nuclear spins in a static magnetic field is the Zeeman interaction, which is between the magnetic dipole moment $\vec{M}=\xi \hbar \vec{I}$ of the spin $\vec{I}$ and the magnetic field $\vec{B}_{0}$,

$$
\hat{H}_{Z}=-\vec{M} \cdot \vec{B}_{0}=-\gamma \vec{B}_{0} \cdot \hbar \hat{\bar{I}}=-\gamma \hbar B_{0} \hat{I}_{z}
$$

The direction of $\vec{B}_{0}$ defines the $z$-axis of the laboratory frame. Equation (2.1) indicates that the Zeeman interaction is determined by the strength of $\vec{B}_{0}$ and the nuclear gyromagnetic ratio $\gamma$. and does not contain information on the chemical and spatial structures of the molecule. The extraction of structure information is based instead on local spin interactions of much smaller magnitudes than the Zeeman interaction. For spin-1/2 nuclei, one of the local spin interactions is the chemical shift,

$$
\hat{H}_{C S}=\xi \hat{\bar{I}} \cdot \sigma \cdot \vec{B}_{0},
$$

where $\sigma$ denotes the chemical shift tensor. Chemical shift arises from the interaction of the nuclear spins with the surrounding electrons. Induced by the $\vec{B}_{0}$ field, the electrons produce a small local field which partially shields the neighboring nuclei from the large external magnetic field. Therefore, the net magnetic field experienced by the nuclear spins is reduced from the Zeeman field $\vec{B}_{0}$ by an amount determined by the geometry of the electronic distribution around the nuclei. This distribution is usually anisotropic (i.e. orientation dependent) with respect to $\vec{B}_{0}$, so the chemical shift tensor $\sigma$ is a second-rank 
tensor. Consequently, the chemical shift frequency depends on the orientation of the molecular segment with respect to the magnetic field. The magnitude of the chemical shift interaction is small relative to the Zeeman interaction. For instance, the maximum isotropic chemical shifts of ${ }^{1} \mathrm{H}$ and ${ }^{13} \mathrm{C}$ spins are about $20 \mathrm{ppm}$ and $200 \mathrm{ppm}\left(1 \mathrm{ppm}=10^{-6}\right)$ of the respective Zeeman interactions.

In contrast to the electron-mediated (i.e. bond-mediated) chemical shift interaction, the dipolar interaction between two nuclear spins $\vec{I}_{1}$ and $\vec{I}_{2}$,

$$
\hat{H}_{D}=-\frac{\mu_{0}}{4 \pi} \gamma_{1} \gamma_{2} \hbar^{2} \frac{1}{r^{3}}\left\{\hat{\bar{I}}_{1} \cdot \hat{\vec{I}}_{2}-3\left(\hat{\bar{I}}_{1} \cdot \frac{\vec{r}}{r}\right)\left(\hat{\bar{I}}_{2} \cdot \frac{\vec{r}}{r}\right)\right\}
$$

occurs directly through space. It is anisotropic and diminishes quickly with the internuclear distance $r$. For two spins separated by $1 \AA$, whose internuclear vector is parallel to the $\vec{B}_{0}$ direction, the maximum strength of the dipolar coupling is $30.2 \mathrm{kHz}$ for ${ }^{13} \mathrm{C}-{ }^{1} \mathrm{H}$ spin pairs. In a high magnetic field of 7 Tesla, this corresponds to about $0.01 \%$ and $0.04 \%$ of the ${ }^{1} \mathrm{H}$ and ${ }^{13} \mathrm{C}$ Zeeman interactions, respectively.

A second type of nuclear spin-spin interaction is the indirect $(J)$ coupling. It is mediated by the electrons in the bonds between the two nuclei. The anisotropic part of $J$ coupling between ${ }^{13} \mathrm{C}$ and ${ }^{1} \mathrm{H}$ is usually very weak compared to the isotropic part so that it can be neglected. The isotropic part of $J$ coupling (i.e. scalar coupling) ranges from 150 $\mathrm{Hz}$ for one-bond $J_{C H}$ to negligibly small for nuclei with low gyromagnetic ratios and separated by multiple bonds. In isotropic liquids, the fast isotropic tumbling of the molecules averages all dipolar couplings to zero, leaving the scalar couplings to be exploited for structure determination. In rigid solids, however, the isotropic $J$ coupling is usually much weaker than the dipolar coupling and chemical shift, so it is mostly irrelevant for structure determination. 
Another NMR interaction is the quadrupolar interaction between a quadrupolar nucleus $(I>1 / 2)$ and its surrounding electric field gradients. Since all nuclei studied in this thesis $\left({ }^{1} \mathrm{H},{ }^{13} \mathrm{C}\right.$ and $\left.{ }^{31} \mathrm{P}\right)$ are spin-1/2 nuclei, the quadrupolar interaction will not be discussed further.

For spin-1/2 nuclei, the Zeeman interaction is orders of magnitude larger than the local spin interactions, so the latter can be treated as perturbations of the former using firstorder perturbation theory. ${ }^{1}$ According to this theory, only spin operators that commute with the Zeeman Hamiltonian are retained, i.e. the spin operators that do not commute with $\hat{H}_{z}$ or $\hat{I}_{z}$ are truncated. The truncated chemical shift Hamiltonian is

$$
\hat{H}_{C S}=\not \hbar \hat{I}_{z} \cdot \sigma_{z z} \cdot B_{0}=-\hbar \omega_{0} \hat{I}_{z} \cdot \sigma_{z z}
$$

where $\omega_{0}=-\gamma_{0}$, and $\sigma_{z z}$ is the $z$ diagonal component of the chemical shift tensor.

The truncated dipolar interaction $\hat{H}_{D}$ differs for homonuclear and heteronuclear coupling. The heteronuclear dipolar coupling between an $I$ spin and a $S$ spin is

$$
\hat{H}_{D}^{I S}=-\frac{\mu_{0}}{4 \pi} \gamma_{I} \gamma_{S} \hbar^{2} \frac{1}{r^{3}} \frac{1}{2}\left(3 \cos ^{2} \theta_{I S}-1\right) \cdot\left(2 \hat{I}_{z} \hat{S}_{z}\right)
$$

where $\theta_{I S}$ is the angle between the internuclear vector and the magnetic field. If the internuclear vector is along $\vec{B}_{0}$, then the spatial coefficients in (2.5) are grouped to form the dipolar coupling constant $\delta_{d}$,

$$
\begin{aligned}
\delta_{d} & =-\frac{\mu_{0}}{4 \pi} \gamma_{I} \gamma_{S} \hbar^{2} \frac{1}{r^{3} \cdot \frac{1}{2}\left(3 \cos ^{2} 0^{\circ}-1\right)} \\
& =-\frac{\mu_{0}}{4 \pi} \gamma_{I} \gamma_{S} \hbar^{2} \frac{1}{r^{3}}
\end{aligned}
$$


In comparison, the truncated homonuclear dipolar interaction between spins $I_{I}$ and $I_{2}$ is

$$
\hat{H}_{D}^{I I}=-\frac{3}{2} \frac{\mu_{0}}{4 \pi} \gamma_{I}^{2} \hbar^{2} \frac{1}{r^{3}} \frac{1}{2}\left(3 \cos \theta_{I I}^{2}-1\right) \cdot\left(2 \hat{I}_{1 z} \hat{I}_{2 z}-\frac{2}{3} \hat{I}_{1} \hat{I}_{2}\right)
$$

The additional factor of $3 / 2$ in the homonuclear dipolar coupling compared to the heteronuclear coupling results from a perturbation calculation of the eigenstates and eigenvalues of the homonuclear dipolar Hamiltonian, ${ }^{2}$ and will not be elaborated here.

\subsection{Semiclassical Description of NMR - Magnetization}

Nuclear spins in the absence of a magnetic field have the same energies irrespective of the orientation of the spin angular momentum in space. In the presence of a magnetic field $\vec{B}_{0}$, this energy degeneracy is lifted: the nuclear spin magnetic moment $\vec{\mu}=\xi \hbar \vec{I}$ interacts with the magnetic field with energy of

$$
E=-\bar{\mu} \cdot \vec{B}_{0}=-\gamma \hbar m_{I} B_{0}
$$

The dot product indicates that only the component of the magnetic moment along the $\vec{B}_{0}$ direction, $\mu_{z}=\gamma \hbar m_{l}$, affects the energy of the nuclear spin. The moment $\mu_{z}$ is quantized, and for spin-1/2 nuclei, adopts two discrete values designated by $m_{I}= \pm \frac{1}{2}$. The $+1 / 2$ state $(\alpha)$ is usually defined as spin magnetic moments aligned with the field, and the $-1 / 2$ state $(\beta)$ as those against the field. The fact that $E_{+1 / 2}<E_{-1 / 2}$ indicates that spin magnetic moments that align with the field have a lower energy than those against the field.

The energy difference between the two spin states is $\Delta E=E_{\alpha}-E_{\beta}=-\gamma \hbar B_{0}$. In a 1 Tesla magnetic field, this energy difference falls into the radiofrequency region $\left(10^{5}-10^{8}\right.$ $\mathrm{Hz}$ ) of the electromagnetic spectrum, according to the Bohr frequency condition $\Delta E=\hbar \omega$. 
To put this energy difference into perspective, we recall that rotational energy levels are spaced apart by $10^{9}-10^{11} \mathrm{~Hz}$ (microwave), while vibrational transitions occur at $10^{11}-10^{14}$ $\mathrm{Hz}$ (infrared). Despite the relatively small energy separation of the nuclear spin states, the spin population of the ground state $n_{\alpha}$ achieves a surplus compared to the population of the excited state $n_{\beta}$. In thermal equilibrium, these two populations are related by the Boltzmann equation

$$
\frac{n_{\alpha}}{n_{\beta}}=e^{-\Delta E / k T}
$$

Thus the population difference is ${ }^{3}$

$$
\frac{n_{\alpha}-n_{\beta}}{n_{\alpha}+n_{\beta}}=\frac{e^{-\Delta E / k T}-1}{e^{-\Delta E / k T}+1} \stackrel{\Delta E<<k T}{\longrightarrow} \approx \frac{-\Delta E / k T}{2}=\frac{\eta \hbar B_{0}}{2 k T}
$$

Since the magnetic moment of each spin is $\gamma \hbar m_{I}$, the total magnetic moment of an ensemble of spins, i.e. the magnetization $\vec{M}_{0}$, is

$$
M_{0}=n_{\alpha} \gamma \hbar m_{I \alpha}+n_{\beta} \gamma \hbar m_{I \beta}=\frac{1}{2} \gamma \hbar\left(n_{\alpha}-n_{\beta}\right)=N B_{0} \cdot \frac{\gamma^{2} \hbar^{2}}{4 k T}
$$

Here the total spin number is written as $N=n_{\alpha}+n_{\beta}$. The nuclear magnetization of one mole of spin-1/2 nuclei is on the order of $10^{-10}$ Joule/Tesla, which is very small compared to optical polarizations.

The classical motion of a spin magnetic moment in a magnetic field is induced by the magnetic torque and constrained by constant total energy and angular momentum:

$$
\frac{d \vec{\mu}}{d t}=\bar{\tau}_{m a g}=\vec{\mu} \times \vec{B}_{0}
$$


The cross product indicates that the magnetic moment moves in a plane perpendicular to both $\vec{B}_{0}$ and itself at a rate $\omega_{0}=-\gamma B_{0}$. In other words, the trajectory of the magnetic moment is a cone around $\vec{B}_{0}$. This motion is the motion of a gyroscope and is called precession (Figure 2.1). The precession of nuclear spins about the magnetic field is called Larmor precession, with the Larmor frequency $\omega_{0}$.

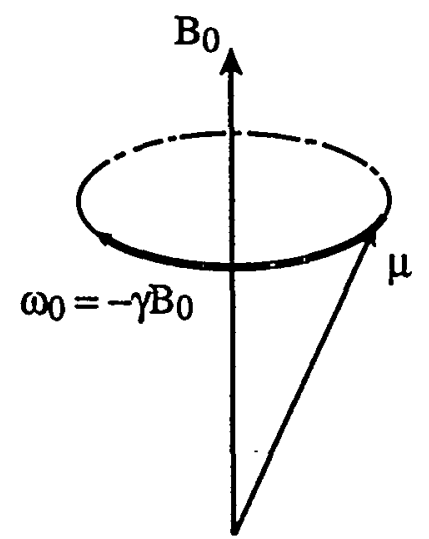

Figure 2.1 Larmor precession of a magnetic moment $\mu$ at rate $\omega_{0}$ in a magnetic field $B_{0}$.

The Larmor precession of the spin magnetic moment is strictly valid only for isolated spins. The total magnetic moments $\vec{M}=\sum \vec{\mu}_{i}$ of a macroscopic sample in equilibrium is along the $\vec{B}_{0}$ direction, so the cross product in equation (2.12) is zero. When the initial magnetization $\vec{M}_{0}$ is not in equilibrium, it moves toward equilibrium according to the precession equation (2.12) with the addition of relaxation,

$$
\frac{d \vec{M}}{d t}=\bar{M} \vec{M} \times \vec{B}-\left(\begin{array}{ccc}
1 / T_{2} & 0 & 0 \\
0 & 1 / T_{2} & 0 \\
0 & 0 & 1 / T_{1}
\end{array}\right)\left(\begin{array}{c}
M_{x} \\
M_{y} \\
M_{z}-M_{0}
\end{array}\right)
$$

Here $\mathrm{T}_{1}$ and $\mathrm{T}_{2}$ are the time constants with which the longitudinal $\left(M_{Z}\right)$ and the transverse ( $M_{x}$ and $M_{y}$ ) components of magnetization relax to their respective equilibrium values, $M_{0}$ 
and 0 . The motion of magnetization without radiofrequency $(\mathrm{rf})$ perturbations is described by these Bloch equations (2.13). When an oscillating rf field is applied perpendicular to the static field $\vec{B}_{0}$, the total magnetic field becomes $\vec{B}=\left(B_{1} \cos \omega t, B_{1} \sin \omega t, B_{0}\right)$, and the solution for the Bloch equations are more complex.

In NMR, the nuclear spin system is probed by an oscillating rf field $\vec{B}_{1}=B_{1} e^{i \omega_{0} t}$, whose oscillation frequency equals the nuclear Larmor frequency. This ensures that the rf field rotates synchronously with the precessing nuclear spins. In a coordinate frame rotating at $\omega_{0}$, the effect of the static field $\vec{B}_{0}$ is thus removed, while the static component of the oscillating rf field $\vec{B}_{1}$ remains. This rotating-frame rf field exerts a torque onto $\vec{M}_{0}$, causing it to precess around $\vec{B}_{1}$ in the rotating frame until the rf field is removed. The precession of $\bar{M}_{0}$ in the rotating frame (i.e. nutation) is manifested as a wobbling of $\vec{M}_{0}$ in its Larmor precession pathway in the laboratory frame. Such a wobbling corresponds to the resonance phenomena in optical spectroscopies.

If the rf field $\vec{B}_{1}$ is removed when $\vec{M}_{0}$ reaches the transverse plane of the rotating frame, then $\vec{M}_{0}$ resumes its Larmor precession about $\bar{B}_{0}$ and induces an oscillating magnetic flux density. A rf coil placed in the plane transverse to $\bar{B}_{0}$ records the voltage induced by this changing flux. Thus experimental NMR measures the change of $\vec{M}_{0}$ rather than $\vec{M}_{0}$ itself. Since this change occurs after the rf irradiation, pulse NMR spectroscopy differs from the absorption or emission processes encountered in electronic and rovibrational spectroscopies.

\subsection{Quantum Mechanical Description of NMR - Density Operator}

The behavior of a statistical ensemble of nuclear spins can be described elegantly by the density operator $\hat{\rho}$, which is an average quantity of spin states $|\psi\rangle$ according to $\hat{\rho}=\mid \overline{\psi\rangle\langle\psi|}$. Represented in the basis $\{|i\rangle\}$ of the state vectors, a density matrix has the elements 


$$
\rho_{i j}=\langle i|\rho| j\rangle=\overline{a_{i}^{*} a_{j}}
$$

where $\left\{a_{i}\right\}$ are the vector coefficients.

The physics of density matrix lies in the difference between its diagonal elements and its off-diagonal elements. ${ }^{1}$ Its diagonal terms $\rho_{i i}$ correspond to the probabilities of finding the spin system in the basis state $|i\rangle$, thus they are called populations. The cross terms $\rho_{i j}$ describe the interference effects between basis states $|i\rangle$ and $|j\rangle$, and are non-zero only when the interference persists through the system. Thus the cross terms are called coherences.

In thermodynamic equilibrium, the density operator is related to the spin Hamiltonians according to

$$
\hat{\rho}=Z^{-1} e^{-\hat{H} / k T},
$$

where $Z$ is the partition function $Z=\operatorname{Tr}\left\{e^{-\hat{H} / k T}\right\}$. In the energy eigenbasis, the density matrix of an equilibrium sppin ensemble has zero coherences. However, the density matrix of a pure spin state, prepared either by an isolated spin or by external perturbations, has non-zero coherences.

In common magnetic field strengths of 1-10 Tesla, even the largest nuclear interaction, the Zeeman interaction, is much smaller than the thermal energy $k T$ for $T \gg$ 1K. As a result, when the exponential in equation (2.15) is expanded into a Taylor series, terms higher than the linear order can be truncated to give

$$
\hat{\rho} \propto\left(\hat{1}+\frac{\not \hbar B_{0}}{k T} \hat{I}_{z}\right)
$$


The unit matrix $\hat{1}$ is unaffected by any spin interactions and can be ignored. For simplicity, the partial density operator $\hat{I}_{z}$ is usually considered without the coefficients in the front. So it should be remembered that the size of the equilibrium density operator is directly proportional to the $\gamma$ of the nuclear spin and the $\vec{B}_{0}$ field strength. The basis for the truncation leading to equation (2.16) is called the high temperature approximation.

The density operator allows the machinery of quantum mechanics to be used for the study of nuclear spin ensembles. For instance, the density operator is related to a quantum mechanical observable $\hat{A}$ according to

$$
\langle\hat{A}\rangle=\operatorname{Tr}\{\hat{\rho} \hat{A}\}
$$

It also describes the time evolution of the spin system as

$$
\frac{d \hat{\rho}}{d t}=-\frac{i}{\hbar}[\hat{H}, \hat{\rho}]
$$

Equation (2.18), called the von Newmann equation, resembles the Heisenberg picture of the Schrödinger equation for the time evolution of state vectors except for the difference in sign. The general solution for the von Newmann equation (2.18) is

$$
\hat{\rho}(t)=\hat{U}(t) \hat{\rho}(0) \hat{U}^{-1}(t)
$$

The unitary operator $\hat{U}$ describes the time propagation of the density operator. For timeindependent Hamiltonians,

$$
\hat{U}(t)=e^{-\frac{i}{\hbar} \hat{H} t}
$$


In NMR, the only observables are the three components of the magnetization vector, $\left.\eta \hbar\left\langle\hat{I}_{x}\right\rangle\left\langle\hat{I}_{y}\right\rangle\left\langle\hat{I}_{z}\right\rangle\right)$. The spin operators for spin-1/2 nuclei $\hat{I}_{\alpha}(\alpha=x, y, z)$ are related to the so-called Pauli matrices $\hat{\sigma}_{\alpha}$,

$$
\hat{\sigma}_{x}=\left(\begin{array}{ll}
0 & 1 \\
1 & 0
\end{array}\right), \hat{\sigma}_{y}=\left(\begin{array}{cc}
0 & -i \\
i & 0
\end{array}\right), \hat{\sigma}_{z}=\left(\begin{array}{cc}
1 & 0 \\
0 & -1
\end{array}\right)
$$

by $\hat{I}_{\alpha}=\frac{1}{2} \hat{\sigma}_{\alpha}$. (From now on the operator symbol ^ will be omitted when the meaning is clear from the context.) The Pauli matrices and the unit matrix $\hat{1}=\left(\begin{array}{ll}1 & 0 \\ 0 & 1\end{array}\right)$ form a basis of the $2 \times 2$ Liouville space, so that any $2 \times 2$ operators can be described by a linear combination of these four matrices.

The nuclear spin operators obey the following commutation rules:

$$
\left[I_{x}, I_{y}\right]=i I_{z}, \quad\left[I_{y}, I_{z}\right]=i I_{x}, \quad\left[I_{z}, I_{x}\right]=i I_{y} .
$$

Consider the evolution of the density matrix under a time-independent Hamiltonian. The propagator equation (2.19) can be expanded to the Baker-Hausdorff series, ${ }^{1}$

$$
\rho(t)=\rho(0)+\sum_{n=1}^{\infty}(-1)^{n} \frac{(i t / \hbar)^{n}}{n !} r_{n}
$$

in which $r_{n}=\left[H, r_{n-1}\right]$ and $r_{0}=\rho(0)$. This infinite commutation series can be simplified to a tractable analytical expression if $r_{2}=c \cdot r_{0}$, since the even and odd terms in the summation can then be grouped separately into a cosine and a sine series. The result is

$$
\rho(t)=\rho(0) \cos (\omega t)-\frac{i / \hbar}{\omega}[H, \rho(0)] \sin (\omega t),
$$


where $(\hbar \omega)^{2}=c$. Equation (2.24) is central for calculating the density matrix evolution under spin Hamiltonians. The oscillatory behavior of the density matrix is reminiscent to the precession of the magnetization vector around the magnetic field. In fact, this oscillation can be derived by considering an initial density matrix $\rho(0)=I_{x}$, prepared, for example, from an equilibrium magnetization by a $90^{\circ}$-y if pulse. The total Hamiltonian, the Zeeman and chemical shift interactions, is linear with $I_{z}$ and recursive according to $r_{2}=\left[-\hbar \gamma B_{0}\left(1-\sigma_{z z}\right)\right]^{2} \cdot r_{0}$. Defining $\omega_{L}=\left(1-\sigma_{z z}\right) \omega_{0}$ as the modified Larmor frequency, we obtain

$$
\left[H_{z}+H_{C S}, \rho(0)\right]=\hbar \omega_{L} \cdot\left[I_{z}, I_{x}\right]=\hbar \omega_{L} \cdot i I_{y}
$$

Substituting this into equation (2.24), we find

$$
\rho(t)=I_{x} \cos \left(\omega_{L} t\right)+I_{y} \sin \left(\omega_{L} t\right)
$$

This is exactly the precession motion of the nuclear magnetization.

The effect of rf pulses on magnetization is considered by combining the propagator equation (2.19) and the commutation relations (2.22). For example, a rf pulse of duration $t_{p}$ along the $-y$ axis affects the density matrix through the propagator

$$
U\left(t_{p}\right)=e^{-i\left(H_{1,-y} / \hbar\right) t_{p}}=e^{-i\left(-\gamma B_{1} I_{-y}\right) t_{p}}
$$

With an initial equilibrium magnetization $I_{z}$, the density matrix after the pulse becomes

$$
\begin{aligned}
\rho\left(t_{p}\right) & =U\left(t_{p}\right) \cdot I_{z} \cdot U^{-1}\left(t_{p}\right) \\
& =e^{i\left(\gamma B_{1} I_{-y}\right) t_{p}} \cdot I_{z} \cdot e^{-i\left(\gamma B_{1} I_{-y}\right) t_{p}} \\
& \ldots(\text { Baker - Hausdorff series })
\end{aligned}
$$




$$
=I_{z} \cos \left(-\gamma B_{1} t_{p}\right)-I_{x} \sin \left(-\gamma B_{1} t_{p}\right)
$$

With a $90^{\circ}$ pulse $\gamma B_{1} t_{p}=90^{\circ}$, thus

$$
\rho\left(t_{p}\right)=I_{x}
$$

The effect of rf pulses on the density matrix is an example of the rotation of angular momentum, which can be summarized by the left-hand rule: if the thumb of the left hand points to the $\vec{B}_{1}$ direction, then the four fingers indicate the sense of the rotation of the angular momentum.

\subsection{Two-Dimensional Fourier NMR}

Modern NMR experiments are almost invariably performed in the pulse mode instead of the continuous wave (CW) mode. In pulse NMR, if pulses on the order of $1 \mu \mathrm{s}$ - $50 \mathrm{~ms}$ are irradiated onto a sample containing the NMR sensitive nuclei. The response of the spin system, which is a complex oscillating signal decaying with time, is then recorded. This Free Induction Decay (FID) is a superposition of the oscillatory responses from magnetically inequivalent nuclei under various spin interactions. The decay of the FID results from spin relaxation to thermal equilibrium, and as such, is an undesirable feature of NMR spectra. To unravel the information content in the complex time signal, we employ the mathematical tool of Fourier transformation, which picks out the frequency components in a time-domain function $f(t)$ to form a frequency spectrum $F(\omega)$,

$$
F(\omega)=\int_{-\infty}^{\infty} f(t) e^{-i \omega x} d t
$$


Fourier transformation is reversible for the Fourier pair $\omega$ and $t$. Thus the time signal $f(t)$ can be obtained from the spectrum $F(\omega)$ by

$$
f(t)=\frac{1}{2 \pi} \int_{-\infty}^{\infty} F(\omega) e^{i \omega t} d \omega .
$$

Pulse Fourier transform (FT) NMR offers many advantages over CW NMR. ${ }^{4}$ First, FT NMR reduces experimental time, since the short rf pulses (e.g. $10 \mu \mathrm{s}$ ) simultaneously excite a range of NMR frequencies (e.g. $100 \mathrm{kHz}$ ) in the molecule of interest. In comparison, in CW NMR, the resonance frequencies are excited incrementally by sweeping the rf field or the magnetic field, thus the $\mathrm{CW}$ experiments are more time consuming. Given the same amount of time, pulse FT NMR provides higher spectral sensitivity than CW NMR. Second, pulse FT NMR allows the implementation of multiplepulse experiments, which are essential for selecting and manipulating various NMR interactions. Multidimensional NMR, which invariably employs multiple rf pulses and which provides rich information on molecular structure and dynamics, is not possible without the methodology of pulse FT NMR.

Table 2.1 lists the Fourier transform of several frequently encountered functions.

\begin{tabular}{|c|c|c|}
\hline$f(t)$ & $\stackrel{F T}{\longrightarrow}$ & $F(\omega)$ \\
\hline delta function: $\delta\left(t-t_{0}\right)$ & & harmonic oscillation: $e^{i \omega_{0}}$ \\
\hline exponential: $e^{-t / \tau}$ & & Lorentzian: $\frac{1 / \tau}{\omega^{2}+1 / \tau^{2}}-i \frac{\omega}{\omega^{2}+1 / \tau^{2}}$ \\
\hline Gaussian: $e^{-t^{2} /\left(2 \sigma^{2}\right)}$ & & Gaussian: $\sqrt{2 \pi} \sigma \cdot e^{-\omega^{2} \sigma^{2} / 2}$ \\
\hline box: $\begin{cases}1, & -t_{0} \leq t \leq t_{0} \\
0, & t<t 0, t>t_{0}\end{cases}$ & & sinc function: $\frac{2 \sin (\omega t 0)}{\omega}$ \\
\hline
\end{tabular}

Table 2.1 Fourier transforms of some common functions. 
Due to the symmetry of Fourier transformation, these relations are retained when the frequency spectrum is converted to the time signal.

Some routine operations in NMR data processing rely on a few basic properties of Fourier transformation. Four such properties are discussed below according to their relevance to the current thesis.

\section{Symmetry Theorem}

A real FID (i.e. with a zero imaginary part) gives a symmetric real spectrum and an antisymmetric imaginary spectrum after Fourier transformation,

$$
f(t)=f^{*}(t) \stackrel{F T}{\longrightarrow} F(\omega)=F^{*}(-\omega) .
$$

Thus a symmetric real spectrum can be achieved by setting the imaginary component of the time signal to zero. For 2D data, the imaginary signal can be zeroed after the first FT with respect to $t_{2}$ to create symmetric lineshapes in $\omega_{1}$. Based on this property, only the cosine component of dipolar coupling frequency needs to be recorded to achieve symmetric dipolar Pake patterns in the $\omega_{1}$ dimension.

\section{Integral Theorem}

Substituting $\omega=0$ in the definition of FT (2.41), we obtain $F(0)=\int_{-\infty}^{\infty} f(t) d t$, indicating that the first point in a spectrum corresponds to the sum FID intensity, and vice versa. Because the first point detected in a FID is often distorted due to instrumental dead times, the Fourier-transformed spectrum can exhibit distorted lineshapes containing negative and dispersive intensities. To avoid the dead time problem, we can delay the detection of the FID by using a spin-echo sequence. 
In 2D NMR, the integral theorem is manifested as the correspondence between the projection and the cross section along the same direction in the time and frequency domains. Specifically, for a projection onto the $\omega_{2}$ axis in the frequency domain,

$$
\begin{gathered}
P\left(\omega_{2}\right) \equiv \int S\left(\omega_{1}, \omega_{2}\right) d \omega_{1} \stackrel{F T}{\longrightarrow} \underset{\text { projection }}{\longrightarrow} f\left(t_{1}=0, t_{2}\right) . \\
\text { cross section }
\end{gathered}
$$

This indicates that the first FID collected in a 2D data set determines the lineshapes of the projection of the $2 \mathrm{D}$ spectrum onto the $\omega_{2}$ axis.

\section{Convolution Theorem}

The convolution of two functions $F(\omega)$ and $G(\omega)$ is defined as

$$
F(\omega) * G(\omega)=\int d \omega^{\prime} F\left(\omega^{\prime}\right) \cdot G\left(\omega-\omega^{\prime}\right)
$$

The Fourier transform of a product of two functions is the convolution of the individual Fourier transforms,

$$
f(t) \cdot g(t) \stackrel{F T}{\longrightarrow} F(\omega) * G(\omega) .
$$

The convolution theorem explains truncation and time apodization in NMR data processing. When a FID is detected for a period shorter than the relaxation time of the signal due to limited storage space, the FID is said to be truncated. The truncated signal can be thought of as the product of the complete FID with a box function of limited width. According to the convolution theorem, the Fourier transform of the truncated FID is the convolution of a Lorentzian line with a sinc function. Thus, sinc wiggles appear at the base of the frequency peak, reducing the spectral resolution. To minimize the truncation effect, 
various window functions can be applied to "apodize" the FID, i.e. to make the signal decay smoothly to zero at the end. When the applied window function is a Gaussian, the Fourier-transformed spectrum also adopts a Gaussian lineshape instead of a Lorentzian one, and the sinc wiggles disappear. While time apodization improves spectral resolution by removing sinc wiggles at the base of the spectral lines, the full-width at half maximum (FWHM) of the lines are increased due to the induced faster decay of the time signal. Therefore line broadening is an inevitable consequence of time apodization, and these two phrases are often used interchangeably.

\section{Shift Theorem}

The shift theorem

$$
f\left(t-t_{0}\right) \stackrel{F T}{\longrightarrow} e^{-i \omega_{0}} \cdot F(\omega)
$$

is the basis for linear phase correction, and is applied, for instance, when a FID has been left-shifted to remove the first data points that are distorted by the instrumental dead times. To a good approximation, the phase of the spectrum after left shift is related to that before by a phase factor linear in frequency, $e^{-i \omega x_{0}}$. However, the loss of information contained in the first few points means that linear phase correction cannot reproduce the original spectrum entirely. It can be shown ${ }^{2}$ that the sinc wiggles appearing at the peak base cannot be removed by linear phase correction. A perfectly-phased spectrum can be achieved instead by detecting the FID with a spin echo sequence. If the detection begins at $t_{0}$ before the echo maximum, and the FID is left-shifted by the same amount before Fourier transformation, then

$$
f^{\prime}(t)=f\left(t+t_{0}\right) \stackrel{F r}{\longrightarrow} F(\omega)=e^{+i \omega_{0}} \cdot F(\omega)
$$


The pure-absorptive spectrum $F^{\prime}(\omega)$ is related to the original phase-distorted spectrum $F(\omega)$ by the linear phase term $e^{+i \omega_{0}}$.

So far, we discussed Fourier transformation mainly in the context of 1D NMR. Two-dimensional Fourier transformation is an extension of the 1D FT,

$$
F\left(\omega_{1}, \omega_{2}\right)=\int d \omega_{2} e^{-i \omega_{2} t_{2}} \int d \omega_{1} e^{-i \omega_{1} t_{1}} f\left(t_{1}, t_{2}\right)
$$

Multidimensional FT NMR allows the correlation and separation of different local spin interactions, which are sensitive to the electronic distribution and consequently the molecular conformation and dynamics. Multidimensional NMR not only can provides a large amount of structural and dynamical information, ${ }^{46}$ but also enhances the resolution of NMR spectra by "spreading out" spectral intensities over a plane, a cube or even higherdimensional geometries.

To analyze 2D NMR spectra correctly, we need to obtain purely absorptive lineshapes in both frequency dimensions. Usually, successive transformations of the 2D time data $f\left(t_{1}, t_{2}\right)=\left\langle e^{i \omega_{1}^{\prime} t_{1}} e^{i \omega^{\prime}{ }_{2} t_{2}}\right\rangle$ cannot generate pure-absorptive spectra, since

$$
F\left(\omega_{1}, \omega_{2}\right)=\left\langle\left(A_{1}+i D_{1}\right)\left(A_{2}+i D_{2}\right)\right\rangle
$$

contains both absorptive (A) and dispersive (D) intensities. This contrasts with 1D spectra, whose real parts can always be phased to give absorptive lineshapes. Therefore, special data acquisition and storage procedures are used in 2D NMR. One procedure is the States method, ${ }^{7}$ which involves separate recording of the cosine- and sine-modulated FIDs,

$$
f_{c}\left(t_{1}, t_{2}\right)=\left\langle\cos \left(\omega_{1}^{\prime} t_{1}\right) e^{i \omega^{\prime}{ }_{2} t_{2}}\right\rangle, f_{s}\left(t_{1}, t_{2}\right)=\left\langle i \sin \left(\omega_{1}^{\prime} t_{1}\right) e^{i \omega^{\prime} t_{2}}\right\rangle
$$


In other words, the FIDs are amplitude-modulated rather than phase-modulated at the end of the $t_{1}$ period. The above cosine and sine signals are Fourier-transformed separately with respect to $t_{2}$. Then the imaginary part of each data set is set to zero, and the mixed frequency-time data are Fourier-transformed with respect to $t_{1}$. The cosine spectrum is

$$
\begin{aligned}
F_{c}\left(\omega_{1}, \omega_{2}\right) & =F T_{1}\left\{\operatorname{Re}\left[F T_{2}\left(f_{c}\left(t_{1}, t_{2}\right)\right)\right]\right\} \\
& =F T_{1}\left\{\operatorname{Re}\left[\cos \left(\omega^{\prime}{ }_{1} t_{1}\right)\left(A_{2}+i D_{2}\right)\right]\right\} \\
& =F T_{1}\left\{\cos \left(\omega^{\prime}{ }_{1} t_{1}\right) A_{2}\right\} \\
& =F T_{1}\left\{\frac{1}{2}\left(e^{i \omega^{\prime}{ }_{1} t_{1}}+e^{-i \omega^{\prime} t_{1}}\right) A_{2}\right\} \\
& =\frac{1}{2}\left(A_{1}\left(\omega-\omega^{\prime}{ }_{1}\right)+A_{1}\left(\omega+\omega^{\prime}\right)\right) A_{2}+\text { imaginary }
\end{aligned} .
$$

Similarly, the sine spectrum is

$$
F_{s}\left(\omega_{1}, \omega_{2}\right)=\frac{1}{2}\left(A_{1}\left(\omega-\omega_{1}^{\prime}\right)-A_{1}\left(\omega+\omega_{1}^{\prime}\right)\right) A_{2}+\text { imaginary }
$$

Combining the two spectra (2.52) and (2.53), we obtain a non-symmetrized and pure-absorptive spectrum $\left\langle A_{1} A_{2}\right\rangle$ with intensities at $\omega_{1}^{\prime}=\omega$. By this means, sign discrimination is achieved in the $\omega_{1}$ frequency dimension analogous to quadrature-detected ID NMR spectra.

It can be seen from (2.52) that the cosine-modulated FIDs alone give rise to pureabsorptive real spectra that are symmetric in $\omega_{1}$. For intrinsically symmetric spectra such as the dipolar Pake patterns, the collection of the cosine data set would then be sufficient. However, most NMR spectra are asymmetric so that off-resonance detection would be necessary if only the cosine-modulated frequences are detected. However, off-resonance detection requires high if powers that are not always available, so on-resonance detection is 
often preferred. To obtain pure-absorptive spectra under on-resonance conditions, the sine data set must be recorded and Fourier-transformed according to equation (2.53).

To optimize the sensitivity and resolution of 2D spectra within a practical time limit, we should be familiar with the factors that determine each. The signal-to-noise ratio (S/N) of a spectrum grows as the square root of the number of scans. This means that the overall $\mathrm{S} / \mathrm{N}$ of a $2 \mathrm{D}$ spectrum is determined by the total acquisition time, not the partition between the number of scans per FID and the number of $t_{1}$ points. The spectral resolution is limited by two inverse relations: the dwell time is the inverse of the spectral width (SW), $t_{d w e l l}=1 / S W$, and the acquisition time is the inverse of the spectral resolution $\left(\Delta \omega_{r s l}\right), t_{a c q}=1 / \Delta \omega_{r s l}$.

\subsection{Powder Lineshapes - Orientation Dependence in Solid-State NMR}

The NMR spectra of polycrystalline solids differ from those of isotropic liquids in two fundamental aspects: the lineshape and the linewidth. The spectra of polycrystalline solids are inhomogeneous patterns tens of kilohertz broad and with characteristic singularities, whereas the liquid spectra consist of sharp Lorentzian lines with widths of a few hertz or less. This difference results from the fact that NMR spin interactions are second-rank tensorial interactions that depend on the orientation of the molecular segment with respect to the magnetic field. 8.9 In isotropic liquids, molecules undergo random rotations at rates fast on the NMR time scales, thereby averaging out the anisotropic (orientation dependent) part of the interactions. ${ }^{10}$ This leaves only the isotropic frequency to be manifested in the spectra. In polycrystalline solids, the relative immobility of the molecules and the random distribution of the crystallite orientations preserve the anisotropic frequencies. The resulting NMR spectrum of each inequivalent site is an inhomogeneously broadened "powder" pattern covering a range of frequencies, each of which corresponds to a different orientation of the molecule. The singularities in the powder pattern correspond 
to specific molecular orientations and can be used to extract structural information about the molecule.

Contrary to polycrystalline solids, the immobile molecules in single crystal solids orient uniformly along a crystal-determined axis. All molecules thus have the same orientation with the magnetic field, and well-resolved lines are manifested in the NMR spectra of single crystals. However, in contrast to isotropic liquids, these sharp lines occur at anisotropic frequencies determined by the orientation of the crystal in the magnet, the orientation of the unit cell with the crystal surface, and the orientation of the molecular segment in the unit cell.

Inhomogeneous powder patterns are also observed in the NMR spectra of $\mathrm{L}_{\alpha^{-}}$ phospholipids, whose bilayers are equivalent to the crystallites in polycrystalline solids. In a hydrated phospholipid sample, bilayer domains are randomly oriented so that a distribution of frequencies is observed in the NMR spectrum. Fast uniaxial motion of the lipid molecules around the bilayer normal narrows the anisotropic width of the spectrum and changes the frequencies of the singularities. However, the powder patterns do not collapse into isotropic lines due to the spatial restriction of the molecular motion.

Just as $\mathrm{L}_{\alpha}$-phospholipids are the motional analog of polycrystalline solids, nematic liquid crystals (LC) can be viewed as the motional analog of single crystals. The nematic LC molecules execute uniaxial motion around a unique sample axis, which is fixed with respect to the magnetic field direction. Thus the NMR spectra of nematic LCs exhibit sharp lines at motionally-averaged anisotropic frequencies.

The second-rank NMR interactions such as chemical shift and dipolar coupling can be transformed between different coordinate systems according to the rules of linear algebra. To obtain the NMR frequency spectrum of a nuclear spin in a static molecule, it is convenient to consider the spin interactions in a molecular frame in which the interaction tensor is fixed. In this segmental frame, the distribution of the crystallite orientations is manifested as the distribution of the $\vec{B}_{0}$ directions. By this coordinate transformation, the 
relative orientation between $\vec{B}_{0}$ and the molecular axes can be described by two polar angles $\theta$ and $\phi$, which are the colatitude and azimuth of $\vec{B}_{0}$ in the molecular frame. Since coordinate transformations do not change the physical interactions, a bilinear identity with respect to the interaction tensor $T$,

$$
\vec{b}^{r} T^{r} \vec{b}^{r, T}=\vec{b}^{s} T^{s} \vec{b}^{s, T}
$$

must hold for any two coordinate systems $r$ and $s$. Here $\vec{b}$ is a unit vector along either $\vec{B}_{0}$ or the main motional axis of the molecules. For example, this bilinear identity is inherent in the truncated chemical shift Hamiltonian,

$$
H_{C S}=-\hbar \omega_{0} \cdot \sigma_{\tilde{z} z}^{L F} \cdot I_{z}=-\hbar \omega_{0} \cdot\left(\vec{b}_{0}^{L F} \sigma^{L F} \vec{b}_{0}^{T, L F}\right) \cdot I_{z}
$$

where $\vec{b}_{0} L F=\left(\begin{array}{lll}0 & 0 & 1\end{array}\right)$ is the unit vector along $\vec{B}_{0}$. The coordinate independence of the bilinear term dictates that the chemical shift Hamiltonian can be transformed from the laboratory frame to the principal axis system (PAS) of the chemical shift tensor,

$$
H_{C S}=-\hbar \omega_{0} \cdot\left(\vec{b}_{0}^{P A S} \sigma^{P A S} \vec{b}_{0}^{T, P A S}\right) \cdot I_{z}
$$

With the polar angles $\theta$ and $\phi$ defining the $\vec{B}_{0}$ orientation in the chemical shift PAS, the chemical shift frequency becomes

$$
\begin{aligned}
\omega_{C S} & =-\omega_{0} \cdot \bar{b}_{0}^{P A S} \sigma^{P A S} \bar{b}_{0}^{T, P A S} \\
& =-\omega_{0} \cdot\left((\cos \phi \sin \theta)^{2} \sigma_{x x}^{P A S}+(\sin \phi \sin \theta)^{2} \sigma_{y y}^{P A S}+(\cos \theta)^{2} \sigma_{z z}^{P A S}\right)^{.}
\end{aligned}
$$

The above relation can be simplified by the following parameters, 


$$
\begin{aligned}
& \sigma_{i s o}=\frac{1}{3} \sum_{\alpha=x, y, z} \sigma_{\alpha \alpha}^{P A S}, \sigma_{\alpha}=\sigma_{\alpha \alpha}^{P A S}-\sigma_{i s o}, \omega_{\alpha}=-\omega_{0} \cdot \sigma_{\alpha} \\
& \text { anisotropy parameter : } \delta=\omega_{z} \\
& \text { asymmetry parameter : } \eta=\frac{\sigma_{y}-\sigma_{x}}{\sigma_{z}}
\end{aligned}
$$

With the geometric parameters $(\theta, \phi)$ and the interacion tensor parameters $\left(\omega_{i s o}, \delta, \eta\right)$, we obtain the frequency spectrum of the chemical shift

$$
\omega(\theta, \phi, \delta, \eta)=\omega_{i s o}+\delta \frac{1}{2}\left(3 \cos ^{2} \theta-1-\eta \sin ^{2} \theta \cos 2 \phi\right)
$$

Three points can be made about this frequency relation. First, the chemical shift spectrum depends explicitly on the orientation distribution of $\vec{B}_{0}$ in the tensor PAS. This distribution is the same for all inequivalent sites in a powder sample. Second, the chemical shift spectrum is affected by the electron density distribution around the nucleus through $\delta$ and $\eta$. Since different chemical sites experience different chemical bonds, i.e. electron densities, their NMR powder spectra have distinct lineshapes. Third, for an axially symmetric $(\eta=0)$ chemical shift tensor, the spectrum only depends on the anisotropy $\delta$ and the polar angle $\theta$.

The two-spin dipolar coupling spectrum can be derived from the same principle. Since the dipolar tensor is uniaxial around the internuclear vector,

$$
D^{P A S}=\left(\begin{array}{ccc}
-\frac{1}{2} D_{z z} & & \\
& -\frac{1}{2} D_{z z} & \\
& & D_{z z}
\end{array}\right)
$$

only the angle $\theta$ between the internuclear vector and $\vec{B}_{0}$ is relevant. In addition, the isotropic component (trace) of the dipolar tensor is zero. As a result, the dipolar coupling of a polycrystalline solid is 


$$
\omega_{d}=\delta_{d} \frac{1}{2}\left(3 \cos ^{2} \theta-1\right)
$$

where $\delta_{d}$ is the coupling constant.

From the frequency dispersions of the chemical shift (2.59) and the dipolar coupling (2.61), we can calculate the intensity distribution (i.e. lineshape) of the spectrum. The problem translates to the calculation of the number of molecules having the same frequency or the same orientation with respect to the magnetic field. Distribution problems of this kind can be solved elegantly by exploiting the equality between the orientation distribution $P(\theta)$ and the frequency distribution $S(\omega),{ }^{2}$

$$
S(\omega(\theta))|d \omega|=P(\theta)|d \theta| .
$$

In powder samples, $P(\theta)=\sin \theta$. With the dispersion relation $|d \theta| /|d \omega|$ obtained from equation (2.59), the lineshape $S(\omega)$ for a uniaxial chemical shift tensor $\eta=0$ is

$$
S(\omega)=\frac{1}{\sqrt{3 \delta \cdot(2 \omega+\delta)}}
$$

The uniaxial powder lineshape has a singularity at $\omega=-\frac{1}{2} \delta$, which corresponds to principal values $\sigma_{y}$ and $\sigma_{x}$ of the chemical shift tensor. It also exhibits a shoulder at the third principal value $\omega_{z}=\delta$. A typical uniaxial chemical shift powder pattern is shown in Figure 2.2a. The degeneracy of $\sigma_{y}$ and $\sigma_{x}$ is removed in a $\eta \neq 0$ powder spectrum (Figure 2.2b), where both $\sigma_{x}$ and $\sigma_{z}$ are manifested as shoulders on each side of the $\sigma_{y}$ singularity.

Since the dipolar interaction is uniaxial and has two symmetric transitions, the dipolar powder pattern (Pake pattern) ${ }^{11}$ is a symmetrized version of the uniaxial chemical 
shift spectrum. The splitting of the two singularities in the Pake pattern (Figure 2.2c) corresponds to the dipolar coupling constant $\delta_{d}$ between the two spins.
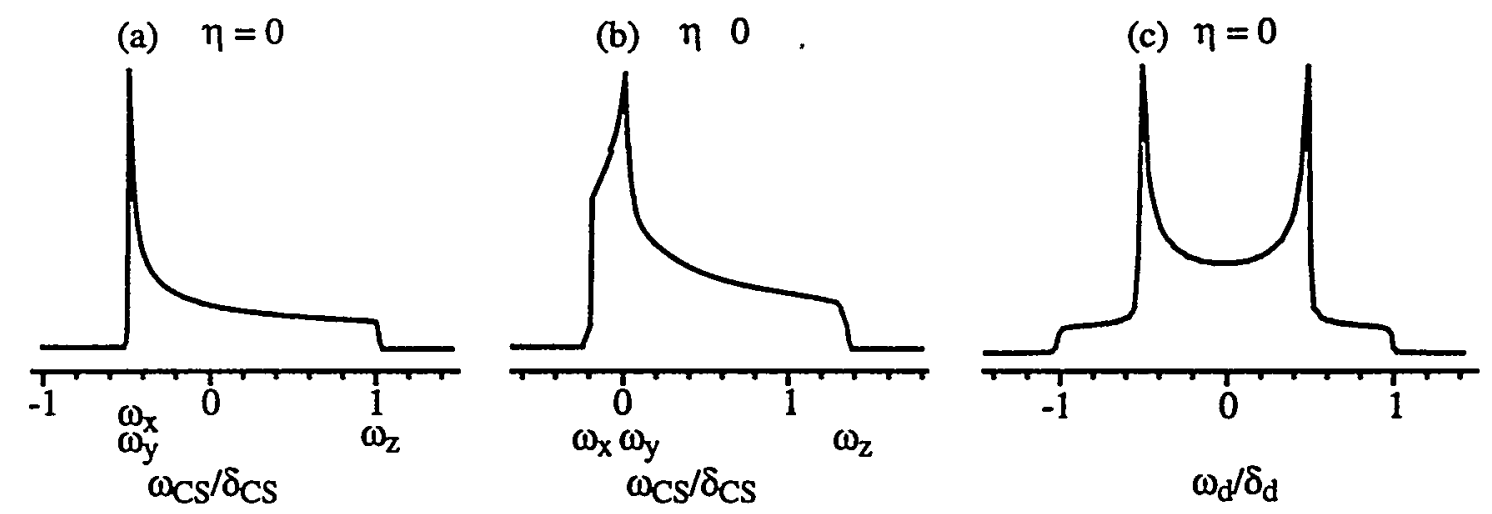

Figure 2.2 NMR powder spectra of polycrystalline solids. (a) Uniaxial chemical shift. (b) Non-uniaxial chemical shift. (c) Heteronuclear dipolar coupling.

\subsection{Sample Spinning - Averaging in Physical Space}

Although NMR powder spectra contain useful information on molecular structure through tensor parameters $\delta$ and $\eta$, the broad resonance lines cause site overlap which obscures such information. Two-dimensional NMR alone may not achieve the necessary site resolution, since even a small organic molecule can have enough inequivalent sites to make the 2D spectra intractable. To obtain high-resolution NMR spectra of solids, the anisotropic frequencies must be either averaged out or scaled to reasonable widths. They can be achieved by spinning the solid sample about an axis inclined at an angle $\psi$ to the magnetic field. ${ }^{12}$ Since variable-angle sample spinning is a common ingredient in the current work, we describe the principles of sample spinning briefly in the following.

Consider the effects of sample rotation on the chemical shift interaction. Under the static condition, the chemical shift spectrum of a polycrystalline solid is a broad powder pattern resulting from the random molecular orientations. In deriving equation (2.59), a 
coordinate transformation from the laboratory frame to the interaction tensor PAS is necessary. When the sample is spun about an axis inclined at an angle $\psi$ with $\vec{B}_{0}$, a new rotor frame (RF) must be considered. Its $z$-axis is defined as the rotor axis and $y$-axis is in the common plane formed by $\vec{B}_{0}$ and the rotor axis. The three coordinate systems are related by Euler rotations

$$
P A S \stackrel{(\alpha, \beta, \gamma)}{\longrightarrow} R F \stackrel{\left(\omega_{r} t, \psi\right)}{\longrightarrow} L F .
$$

Since the rotor frame and the laboratory frame are both uniaxial, only two Euler angles are necessary for the transformation between the two: $\omega_{r} t$ is the azimuth angle and $\psi$ is the colatitude or the spinning angle. Sample rotation imposes a transformation of the segmentfixed chemical shift PAS to the rotor frame common to all molecules, and subsequently a uniform Euler transformation to the laboratory frame. Thus the spinning angle $\psi$ affects all molecules through the second transformation. The successive coordinate transformations of (2.64) give rise to the following time-dependent chemical shift $\omega(t),{ }^{12}$

$$
\begin{aligned}
& \omega(t)=\omega_{i s o}+\frac{1}{2}\left(3 \cos ^{2} \psi-1\right) \delta . \\
& \frac{1}{2}\left(3 \cos ^{2} \beta-1-\eta \sin ^{2} \beta \cos 2 \gamma\right)+\sqrt{\frac{3}{2}} \delta \cdot \xi(t)
\end{aligned}
$$

where the time-dependent function $\xi(t)$ is

$$
\xi(t)=g_{1} \cos \left(\omega_{r} t+\chi_{1}\right)+g_{2} \cos \left(2 \omega_{r} t+\chi_{2}\right) .
$$

Here $g_{1}$ and $g_{2}$ are geometric factors that depend on the parameter set $(\beta, \gamma, \psi, \eta)$, while the phase angles $\chi_{1}$ and $\chi_{2}$ are functions of $(\alpha, \beta, \gamma, \eta)$. As can be seen, the timedependent chemical shift contains three parts. The isotropic part is unaffected by spinning and has the same frequency as in the static sample. The anisotropic component is scaled by 
the product of the second Legendre polynomial, $P_{2}(\cos \psi)=\frac{1}{2}\left(3 \cos ^{2} \psi-1\right)$, with an orientation factor. This factor reflects the distribution of the molecules in the rotor frame analogous to the expression for the static chemical shift (2.59) except for the different Euler angles. The averaged anisotropy $\bar{\delta}$ is defined as

$$
\bar{\delta}=\delta \frac{1}{2}\left(3 \cos ^{2} \beta-1-\eta \sin ^{2} \beta \cos 2 \gamma\right)
$$

The third component in equation (2.65) varies sinusoidally with the rocation period, and gives rise to rotational echoes in the FIDs and spinning sidebands in the spectra. The origin of the rotational echoes lies in the self commutation of the chemical shift Hamiltonian. ${ }^{12}$ As the sample rotates, each crystallite sweeps through a range of orientations (in the laboratory frame) and thus experiences a range of frequencies. Destructive interference of the crystallite phases causes fast decay of the FID during each rotor period. However, the average anisotropic frequency after a complete rotor period is zero, since the chemical shift Hamiltonian commutes with itself at different times. Thus the chemical shift frequencies add up constructively after each rotcr period, and rotational echoes are created. The Fourier transform of a train of echoes is a series of peaks (i.e. sidebands) spaced apart by the rotor frequency. The intensity envelope of the sidebands corresponds to the static powder pattern. Spinning sidebands can be suppressed by rotorsynchronized sampling at the maxima of rotational echoes, or by special if pulse techniques such as TOSS. ${ }^{13}$ The total intensity of the sidebands relative to the centerband decreases also with increasing spinning speeds. In liquid-crystalline phospholipids, the chemical shift anisotropies and dipolar couplings are reduced from the rigid-lattice values, thus even at moderate spinning speeds ( $\sim 2 \mathrm{kHz}$ for ${ }^{13} \mathrm{C} \mathrm{NMR}$ ) the sidebands are removed from the spectra.

The spinning-angle dependence of the NMR spectrum indicates that at the magic angle $\psi=54.7^{\circ}$, where $P_{2}(\cos \theta)$ vanishes, the anisotropic chemical shift is averaged out 
and only the isotropic frequency remains in the spectra. By isotropically narrowing the lines, magic-angle spinning (MAS) achieves site resolution for complex solids with multiple sites. If the sample is spun at an angle away from the magic angle, then the anisotropic width is scaled by a factor $P_{2}(\cos \theta)$. Variable-angle spinning has the advantage of retaining the structural information contained in the anisotropy and asymmetry parameters, in addition to partially enhancing the spectral resolution.

Chemical shift and heteronuclear dipolar coupling are both inhomogeneous in that they commute with themselves at different times of a rotation period. ${ }^{12}$ This explains why they can be averaged out even when the spinning frequency is smaller than the interaction strength. The homonuclear dipolar interaction, however, does not commute with itself, thus it cannot be averaged out by MAS when the spinning speed is smaller than the coupling strength.

\subsection{Average Hamiltonian Theory - Averaging in Spir Space}

Macroscopic sample rotation is one of two ways of averaging the NMR interactions. The spin Hamiltonians can be separated into two parts. A spatial part contains various physical constants (e.g. the gyromagnetic ratio $\gamma$ and the vacuum permeability $\mu_{0}$ ), geometric parameters (e.g. the internuclear distance $r$ and orientation $\theta, \phi$ ) and tensor parameters (e.g. the chemical shift anisotropy $\delta$ ). A spin part contains nuclear spin operators, which are the Pauli matrices for spin-1/2 nuclei. Sample spinning averages the spatial part of the Hamiltonians, whereas if pulses coherently average the spin part of the Hamiltonians.

The average Hamiltonian theory (AHT) describes the effects of rf pulses on the nuclear spin system. ${ }^{8}$ The if pulses can narrow the NMR spectra by averaging the spin part of the Hamiltonians. They can also create new spin dynamics such as cross polarization. Modern NMR experiments commonly employ multiple rf pulses, whose effects on the spin system often cannot be explained by the magnetization trajectory in the 
rotating frame. These aspects of rf irradiation can be understood in the framework of AHT. $^{2,8}$

In the average Hamiltonian theory, local spin interactions that are perturbed by if pulses are viewed in an "interaction space" which removes the immediate effect of the pulses. To a first approximation, the spin Hamiltonian in a period $t_{c}$ equals the average Hamiltonian in the interaction space. This approximation is valid as long as the rf field strength greatly exceeds the strength of the local fields being averaged. If this condition

- does not hold, then higher order averages involving commutations of Hamiltonians must be considered.

A rf pulse affects the local Hamiltonians by rotating the spin operators according to the commutation rules of angular momenta. The rotation can be viewed alternatively as a transformation of the spin operators from the rotating frame, whose unique axis is the static magnetic field and whose frequency is the Larmor frequency, to a frame that "toggles" with the if pulses. In this toggling frame, the effect of the rf pulses becomes invisible, in the same way as the frequency modulation of if pulses is invisible in the rotating frame. ${ }^{2}$ Mathematically, the toggling frame is defined by unitary transformations of the rotatingframe Hamiltonian $H_{R}$ and density operator $\rho_{R}$,

$$
\begin{aligned}
& H_{T}(t)=P^{-1}(t) H_{R} P(t) \\
& \rho_{T}(t)=P^{-1}(t) \rho_{R}(t) P(t)
\end{aligned},
$$

where the subscripts $T$ and $R$ indicate the toggling frame and the rotating frame, respectively. The pulse propagator $P(t)$ is equal to $\prod_{i} e^{-i H_{1 \alpha} t_{i}}(\alpha=x, y, z)$. In this interaction representation (2.68), the time evolution of the density operator is

$$
\dot{\rho}_{T}=-i\left[H_{T}(t), \rho_{T}(t)\right]
$$


Its solution is the familiar unitary transformation

$$
\rho\left(t_{c}\right)=U_{T}\left(t_{c}\right) \rho(0) U_{T}^{-1}\left(t_{c}\right)
$$

in which $U_{T}\left(t_{c}\right)$ is the product of the pulse propagators from $t=0$ to time $t_{c}$ :

$$
U_{T}\left(t_{c}\right)=e^{-i H_{T}\left(t_{c}-t^{\prime}\right) t^{\prime}} e^{-i H_{T}\left(t_{c}-2 t^{\prime}\right) t^{\prime}} \cdots e^{-i H_{T}\left(t^{\prime}\right) t^{\prime}} e^{-i H_{T}(0) t^{\prime}}
$$

The rf Hamiltonian $H_{R}$ does not appear in the propagator as a result of the transformation into the toggling frame. Since the toggling-frame Hamiltonians at different times usually do not commute, the product of the exponentials in (2.71) is not equal to the exponential of the sum of the Hamiltonians $H_{T}\left(t_{c}-n t^{\prime}\right)$. However, this product can be equated with the exponential of a sum of average toggling-frame Hamiltonians,

$$
U_{T}\left(t_{c}\right) \equiv e^{-i H_{T, e f f} t_{c}}=e^{-i\left(\bar{H}^{(0)}+\bar{H}^{(1)}+\bar{H}^{(2)}+\ldots\right) t_{c}}
$$

The various orders of the average Hamiltonians $\bar{H}^{(n)}(n=0,1,2 \ldots)$ in this so-called Magnus expansion are 8

$$
\begin{aligned}
& \bar{H}^{(0)}=\frac{1}{t_{c}} \int_{0}^{t_{c}} H_{T}\left(t^{\prime}\right) d t^{\prime} \\
& \bar{H}^{(1)}=\frac{-i}{2 t_{c}} \int_{0}^{t_{c}} d t_{2} \int_{0}^{t_{2}} d t_{1}^{\prime}\left[H_{T}\left(t_{2}^{\prime}\right), H_{T}\left(t_{1}^{\prime}\right)\right] \\
& \bar{H}^{(2)}=\frac{-1}{6 t_{c}} \int_{0}^{t_{c}} d t_{3^{\prime}} \int_{0}^{t_{3}} d t_{2}^{\prime} \int_{0}^{t_{2}} d t_{1}^{\prime}\left(\left[H_{T}\left(t_{3}^{\prime}\right),\left[H_{T}\left(t_{2}^{\prime}\right), H_{T}\left(t_{1}^{\prime}\right)\right]\right] .\right. \\
& \left.\quad+\left[H_{T}\left(t_{1}^{\prime}\right),\left[H_{T}\left(t_{2}^{\prime}\right), H_{T}\left(t^{\prime}\right)\right]\right]\right)
\end{aligned}
$$

etc. 
In many cases, the zero-order average $\bar{H}^{(0)}$, which is the simple sum of the toggling frame Hamiltonians in the period $t_{c}$, is sufficiently close to the total effective Hamiltonian $H_{T, e f f}$. In other words, $\bar{H}^{(0)}$ dominates all higher order averages combined: $\bar{H}^{(0)} \gg \sum_{n=1}^{\infty} \bar{H}^{(n)}$. To obtain the average Hamiltonian, we calculate the $H_{T}$ 's using equation (2.68), and add up all $H_{T}$ s in the period $t_{c}$. Since a unitary transformation $P^{-1} P$ is equivalent to a left-hand rotation, we can also visualize the toggling-frame Hamiltonians by rotating successive toggling frames about the axes of the rf pulses (in the rotating frame) and recording the axes of the toggling frames that are along the $z$-axis of the rotating frame. The integration limit $t_{c}$ is determined by the specific if pulse sequence. For cyclic multiplepulse sequences, $t_{c}$ is the cycle period, during which the sum of $H_{T}$ 's either vanishes or is considerably simplified. For continuous wave decoupling, $t_{c}$ is either the nutation period $t_{c}=2 \pi / \omega_{1}$ at which $U\left(t_{c}\right) \cong e^{-i \bar{H}^{(0)} t_{c}}=0$, or a long time at which the time propagator approaches zero.

The higher-order terms in the Magnus expansion (2.72) result from the fact that the toggling-frame Hamiltonians at different times do not commute. When $\bar{H}^{(0)} \leq \bar{H}^{(1)}$, or when $\bar{H}^{(0)}=0$ and $\bar{H}^{(n)}(n>0)$ are not sufficiently small, the zero-order Hamiltonian fails to approximate the total effective Hamiltonian, and the higher-order terms must be considered. For example, the first-order average Hamiltonian for heteronuclear decoupling is

$$
\bar{H}^{(1)}=\delta_{d} \frac{\delta_{d}}{2 \omega_{1}} \cdot I_{x}
$$

If the decoupling field $\omega_{1}$ is smaller than the coupling constant $\delta_{d}$, then $\bar{H}^{(1)}$ is nonnegligible and contributes to the total effective Hamiltonian. Therefore, the rf nutation period $t_{c}=2 \pi / \omega_{1}$ must be short compared to the dipolar modulation period $\tau_{d}=2 \pi / \delta_{d}$ in order for the $\bar{H}^{(0)}$ approximation to be valid. 
In the following, we consider three applications of AHT.

\section{Heteronuclear Decoupling}

When constant-phase if pulses with strength $\omega_{1}=-\gamma B_{1}$ are applied continuously on an abundant spin $I$, the dipolar coupling between spin $I$ and the rare spin $S$ can be calculated as

$$
\begin{aligned}
& H_{D}^{I S}=\delta_{d} I_{z} S_{z} \stackrel{I_{1 x}}{\longrightarrow} \\
& \bar{H}_{D}^{I S,(0)}=\delta_{d} \cdot \int_{0}^{2 \pi / \omega_{1}} d t\left(I_{z} \cos \omega_{1} t+I_{y} \sin \omega_{1} t\right) S_{z}=0
\end{aligned}
$$

Because of the sinusoidal time dependence of the spin operators brought about by the continuous rf irradiation, the average $I-S$ dipolar coupling vanishes after a complete nutation period (and at long decoupling times $t \rightarrow \infty$ ). With the dipolar couplings averaged out, the resolution of the $S$-spin spectra is improved.

Similarly, it can be shown that the continuous rf pulses (spin lock) also average out the chemical shift due to the linear dependence of the chemical shift Hamiltonian on the spin operator. In contrast, the nonlinear homonuclear dipolar interaction $H_{D}^{I I} \propto \sum_{j, k}\left(3 I_{z}^{j} I_{z}^{k}-I^{j} I^{k}\right)$ cannot be averaged out. Specifically, with a $+x$ spin lock pulse, $I_{z}$ terms can be integrated to result in a non-zero average Hamiltonian

$$
\bar{H}_{D}^{I I,(0)}=-\frac{1}{2} H_{x}^{I I} \propto \sum_{j, k}\left(3 I_{x}^{j} I_{x}^{k}-I^{j} I^{k}\right)
$$

Since the residual Hamiltonian commutes with $I_{x}$, the magnetization component along the locking field is unaffected by the pulses, i.e. the spin is "locked". Meanwhile, the component perpendicular to the locking field dephases with half the frequency of the homonuclear coupling. 


\section{MREV-8 Homonuclear Decoupling}

The homonuclear decoupling sequence MREV-8 14,15 consists of eight cyclic pulses:

$$
\{\tau,-x, \tau, y, \tau, \tau,-y, \tau, x, \tau, \tau, x, \tau, y, \tau, \tau,-y, \tau,-x, \tau\}
$$

The corresponding toggling-frame homonuclear Hamiltonians are

$$
\left\{H_{z}^{I I}, H_{y}^{I I}, H_{x}^{I I}, H_{x}^{I I}, H_{y}^{I I}, H_{z}^{I I}, H_{z}^{I I}, H_{y}^{I I}, H_{x}^{I I}, H_{x}^{I I}, H_{y}^{I I}, H_{z}^{I I}\right\}
$$

The average homonuclear Hamiltonian after each cycle is

$$
\bar{H}_{D}^{I I,(0)}=4\left(H_{x}^{I I}+H_{y}^{I I}+H_{z}^{I I}\right)=4 \sum_{\alpha=x, y, z}\left(3 I_{\alpha}^{j} I_{\alpha}{ }^{k}-I^{j} I^{k}\right)=0
$$

Therefore, the MREV-8 sequence averages out, in first order, homonuclear dipolar couplings at the end of each cycle. However, the chemical shift interaction is not averaged to zero:

$$
\bar{H}_{c s}^{(0)}=4\left(H_{z}+H_{x}\right) \propto\left(I_{z}+I_{x}\right)
$$

This residual chemical shift causes the magnetization to evolve around an effective field $\vec{B}_{\text {eff }}$ along the diagonal of the $x z$ plane. The magnitude of $\vec{B}_{\text {eff }}$ is scaled by a factor $\sqrt{2} / 3 \approx 0.47$ compared to the chemical shift local field in the absence of MREV-8. To ensure that the magnetization evolves on a circular trajectory around $\vec{B}_{\text {eff }}$, the initial magnetization must be placed perpendicular to $\vec{B}_{\text {eff }}$ by a $45^{\circ}$ rf pulse. Alternatively, a $45^{\circ} x$ 
pulse can be applied before the MREV-8 multiple-pulse sequence so that its effective field is moved to the $z$-axis of the rotating frame.

\section{Hartman-Hahn Cross Polarization}

Cross polarization is a technique to enhance the spectral sensitivity of rare spins $S$ with a low gyromagnetic ratio by transferring polarization from abundant spins $I$ with a high gyromagnetic ratio. ${ }^{16}$ Initially, the $l$-spin coherence is created in the transverse plane by a $90^{\circ}$ pulse while the $S$-spin polarization remains in equilibrium. Next, spin-lock pulses are applied simultaneously on $I$ and $S$, with the strengths of the locking fields fulfilling the Hartman-Hahn condition, ${ }^{17}$

$$
\gamma_{I} B_{1 I}=\gamma_{s} B_{1 s} .
$$

Under the double spin locks, the heteronuclear coupling is averaged to

$$
\begin{aligned}
\bar{H}_{D}^{I S,(0)} & =\omega_{I S} \cdot \int_{0}^{2 \pi / \omega_{1}} d t\left(I_{z} \cos \omega_{1} t+I_{y} \sin \omega_{1} t\right)\left(S_{z} \cos \omega_{1} t+S_{y} \sin \omega_{1} t\right) \\
& =\omega_{I S} \cdot\left(I_{z} S_{z}+I_{y} S_{y}\right)
\end{aligned}
$$

This result requires the $I$ and $S$ spins to have the same nutation frequency $\omega_{1}=\gamma_{I} B_{1 I}=$ $\gamma_{s} B_{1 s}$. Due to the cross term $I_{y} S_{y}$, the $I_{x}$ magnetization evolves into

$$
\begin{aligned}
I_{y} \stackrel{\bar{H}_{D}^{I S,(0)}}{\longrightarrow} & I_{x} \frac{1}{2}\left(1+\cos \omega_{I S} t\right)+S_{x} \frac{1}{2}\left(1-\cos \omega_{I S} t\right) . \\
& +\left(I_{y} S_{z}-I_{z} S_{y}\right) \sin \omega_{I S^{t}}
\end{aligned}
$$

In the final density matrix, observable $S$ spin coherence is present, indicating that the $I$ spin polarization $\gamma_{I} B_{0} / k T$ is transferred to the $S$ spin. The $S$-spin spectrum thus obtained is 
$\gamma_{I} / \gamma_{S}$ times more sensitive than the spectrum from the equilibrium $S$ polarization. For instance, a maximum sensitivity enhancement of 4 is gained on ${ }^{13} \mathrm{C}$ by cross polarization from ${ }^{1} \mathrm{H}$.

We point out that the maximum CP enhancement factor $\gamma_{I} / \gamma_{S}$ is achieved only when the number of $I$ spins is much larger than the number of $S$ spins, ${ }^{16}$ as in the case for natural abundance ${ }^{1} \mathrm{H}(100 \%)$ and ${ }^{13} \mathrm{C}(1 \%)$ nuclei. When the $I-S$ number ratio approaches one as a result of, for example, isotopical enrichment of the $S$ spins, then the enhancement factor is reduced. Take for example a system containing $50 \%$-labeled ${ }^{13} \mathrm{C}$ spins. $\mathrm{CP}$ from ${ }^{1} \mathrm{H}$ to ${ }^{13} \mathrm{C}$ enhances the ${ }^{13} \mathrm{C}$ spectral sensitivity only by a factor of 2.67 . Intuitively, this reduction is due to the fact that the equilibrium transverse polarization per spin after CP must be the same for all spins. With a fixed number of $I$ spins, the more $S$ spins, the smaller the average polarization. In other words, the larger the $I-S$ number ratio, the larger the $S$ spin enhancement factor, until the upper limit of $\gamma_{I} / \gamma_{S}$ is reached.

In practice, cross polarization enhances the sensitivity of the rare spin spectra not only through the favorable $\gamma$ of the abundant spin. The spin-lattice relaxation rates of protons are usually faster than those of carbons due to spin diffusion among the protons. Thus, shorter recycle delays can be used in the CP experiments compared to the singlepulse experiments. This constitutes another pathway of sensitivity enhancement in ${ }^{13} \mathrm{C}$ NMR.

\subsection{Proton-Detected Local Field Spectroscopy}

One of the most important NMR techniques developed and employed in the current work is the proton-detected local field (PDLF) spectroscopy. ${ }^{18-21}$ The technique correlates heteronuclear dipolar couplings involving protons with the chemical shift of the heteronucleus (e.g. ${ }^{13} \mathrm{C}$ and ${ }^{31} \mathrm{P}$ ). In a broad sense, it resembles the separated local field (SLF) spectroscopy first demonstrated by Waugh and coworkers. ${ }^{22-24}$ However, the 
PDLF technique has important differences from and advantages over the traditional SLF technique.

Figure 2.3a shows the PDLF pulse sequence for measuring $S-I$ dipolar coupling, where $S$ represents the spin with a low number density and $I$ is an abundant spin species. The small $S$-spin density can result from low natural abundance of the nucleus or a lack of many inequivalent sites in the system of interest. For example, the $100 \%$ naturally abundant ${ }^{31} \mathrm{P}$ nucleus has only one site per phospholipid molecule and is thus treated as a rare spin. During the evolution period $t_{1}$, the ${ }^{1} \mathrm{H}$ magnetization evolves under the $S-{ }^{1} \mathrm{H}$ dipolar and scalar $(J)$ interactions. The MREV-8 multiple-pulse sequence ${ }^{14,15}$ suppresses the effects of the $\mathrm{H}-\mathrm{H}$ homonuclear coupling, whereas two simultaneous $180^{\circ}$ pulses on ${ }^{1} \mathrm{H}$ and the $S$ spin in the middle of $t_{1}$ refocus the ${ }^{1} \mathrm{H}$ chemical shift while retaining the $S-{ }^{1} \mathrm{H}$ couplings. At the end of the $t_{1}$ period, a ${ }^{1} \mathrm{H}$ spin lock of about $1 \mathrm{~ms}$ selects the cosinemodulated dipolar (and scalar) coherence, which is then transferred to the $S$ spin by Hartmann-Hahn cross polarization. ${ }^{16,17}$ In the $t_{2}$ period, the $S$ magnetization evolves under the chemical shift interaction and is detected in the presence of proton decoupling. From this pulse sequence, we see that "detection" in the term PDLF refers to the detection of dipolar local fields in the indirect dimension. It should not be confused with the final detection of the rare spin NMR signals in the direct dimension of the $2 \mathrm{D}$ experiment.

Figure $2.3 \mathrm{~b}$ shows the pulse sequence of the SLF experiment. Again, the ${ }^{1} \mathrm{H}$ homonuclear couplings and the $S$-spin chemical shift are averaged out during the $t_{1}$ period, and the $S$ chemical shift is detected in the $t_{2}$ period. However, the SLF sequence differs from the PDLF sequence in that the $S{ }^{-1} \mathrm{H}$ dipolar evolution occurs on the rare spin $S$ during $t_{1}$, either by direct excitation of the $S$ magnetization or after polarization transfer from the ${ }^{1} \mathrm{H}$ spins.

Because of the high number density of protons (100\%) and the rare occurrence of the $S$ spins, each proton is practically coupled to only one $S$ spin, whereas each $S$ spin usually couples significantly to several protons. Thus the proton local field consists of 
isolated spin pairs whereas the $S$ local field is that of a multiple-spin system. Since the PDLF technique probes the proton dipolar field, the PDLF spectra should exhibit one dipolar splitting for each $S-{ }^{1} \mathrm{H}$ pair. As a result, the dipolar dimension of a 2D PDLF spectrum for a $S$ spin coupled to $N$ protons exhibits $2 N$ lines (i.e. $N$ splittings), which are correlated with the $S$ chemical shift in the other dimension. These $2 N$ lines are either sharp peaks in the case of oriented samples, or inhomogeneously broadened Pake patterns in the case of unoriented powder samples.
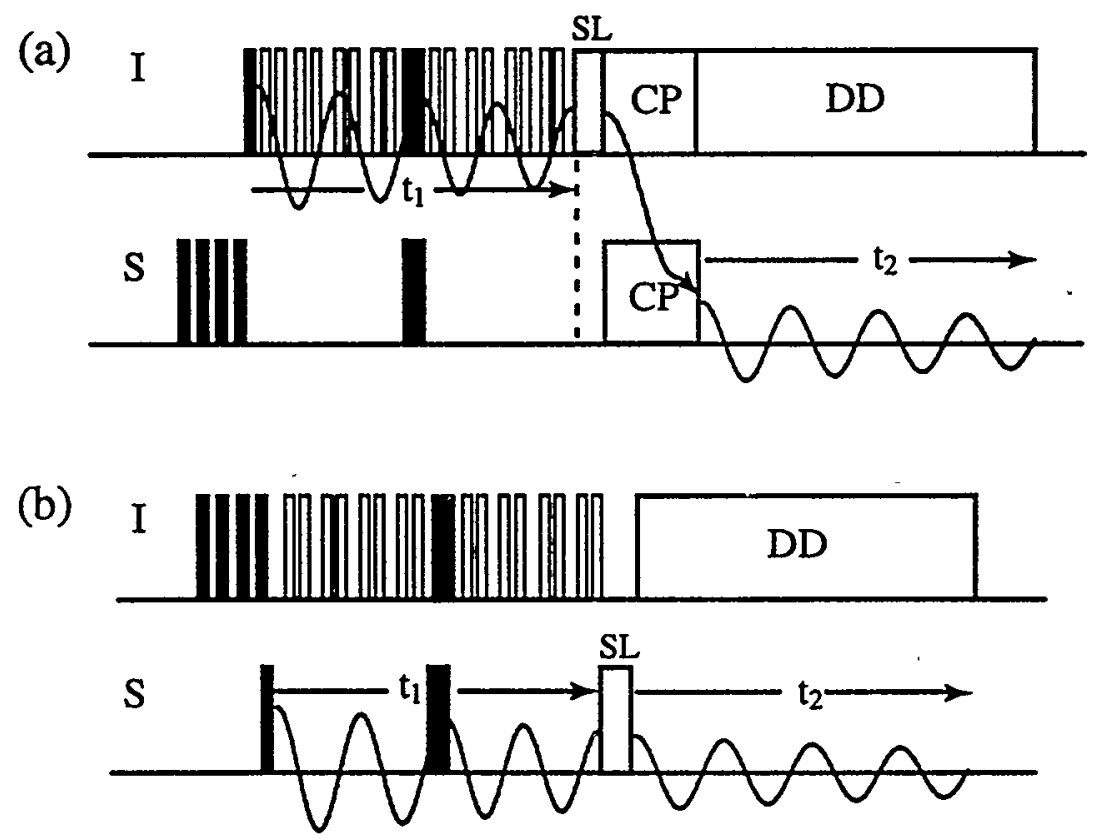

Figure 2.3 (a) PDLF and (b) SLF pulse sequences.

Although the traditional SLF spectroscopy ${ }^{22,23}$ also separates the $S-{ }^{1} \mathrm{H}$ dipolar couplings according to the $S$ chemical shifts, it does so by probing the local field of the $S$ spins, each of which is coupled to $N$ protons that are either directly-bonded or unbonded to it. The successive splittings of a $S$ resonance by the $N$ coupled protons give rise to a dipolar spectrum with a maximum of $2^{N}$ lines. Thus the traditional SLF spectra are more crowded and are broadened by long-range couplings, and it is difficult to extract individual dipolar couplings quantitatively. 
The different complexities between the PDLF and SLF spectra, shown schematically in Figure 2.4, are particularly relevant for extracting long-range dipolar couplings between unbonded nuclei. Long-range dipolar couplings provide constraints on the conformation and orientational order of motionally-averaged molecules. The simplicity of the PDLF spectra enables these small dipolar couplings to be resolved, whereas the large number of lines in the SLF spectra hampers their determination.

(a)
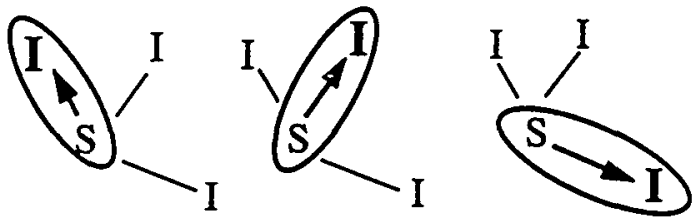

$2 \mathrm{~N}$ lines

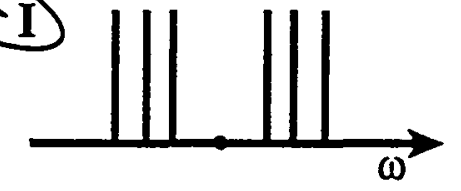

(b)
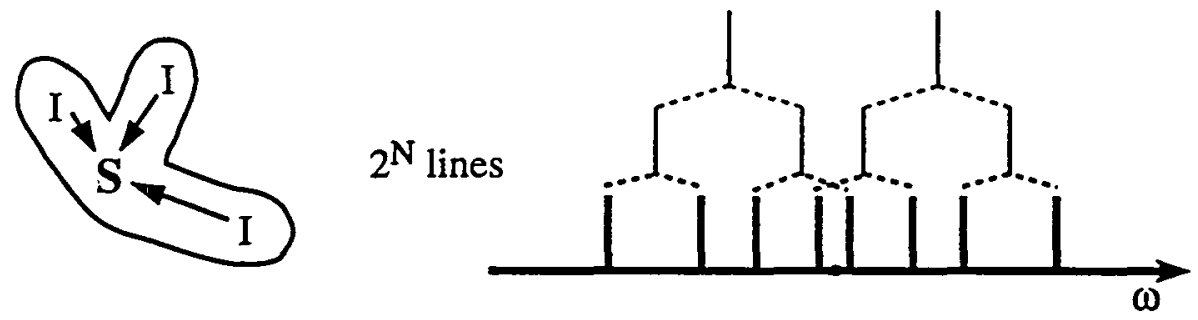

Figure 2.4 Schematic (a) PDLF and (b) SLF stick spectra. Each rare spin $S$ is coupled to $N=3$ abundant spins $I$.

Furthermore, the PDLF dipolar detection scheme (of ${ }^{1} \mathrm{H}$ local fields) and the simplicity of the resulting spectra allow the identification of ${ }^{1} \mathrm{H}$ coupling partners in $S-{ }^{1} \mathrm{H}$ spin pairs. In the SLF experiments, ${ }^{1} \mathrm{H}$ chemical shift frequencies are not measured. The assignment of the ${ }^{1} \mathrm{H}$ spin in a $S-{ }^{1} \mathrm{H}$ coupling is usually achieved on the basis of $S$ chemical shift and geometric arguments. However, for long-range $S{ }^{1} \mathrm{H}$ dipolar couplings in mobile molecules, the ${ }^{1} \mathrm{H}$ identity cannot be determined because the size of the coupling is not predictable a priori. Obviously, the assignment can be made if additional information on ${ }^{1} \mathrm{H}$ chemical shift can be obtained. Indeed, a 3D experiment correlating $S-{ }^{1} \mathrm{H}$ dipolar 
coupling, $S$ chemical shift, and ${ }^{1} \mathrm{H}$ chemical shift can be expected to fulfill this requirement. However, such a correlation involving the ${ }^{1} \mathrm{H}$ chemical shift is not possible by a SLF experiment, because dipolar evolution must occur on the ${ }^{1} \mathrm{H}$ spins in order to relate the ${ }^{1} \mathrm{H}$ chemical shift with the observed $S-{ }^{1} \mathrm{H}$ dipolar couplings. Only the PDLF technique can provide the ${ }^{1} \mathrm{H}$ chemical shift information necessary for the assignment of coupling partners in long-range S-1H couplings. The application of the PDLF technique in both the measurement and assignment of long-range dipolar couplings is demonstrated on a nematic liquid crystal in chapter 7.

The PDLF spectra can be made symmetric in the dipolar dimension by detecting only the cosine component of the dipolar coherence. Normally, both the cosine- and sinemodulated frequencies are required to discriminate the sign of the anisotropic NMR spectra. However, due to the intrinsic symmetry of the dipolar Pake pattern, the sine component of the dipolar coherence contains nearly vanishing signals. Therefore, we only detect cosinemodulated dipolar coherence, and the resulting PDLF spectra are symmetrized in the $\omega_{1}$ dimension.

Note that in the indirect dimension of the PDLF experiment, the isotropic scalar couplings are retained as well as the anisotropic dipolar couplings. For directly-bonded $S$ and ${ }^{1} \mathrm{H}$ nuclei with relatively small dipolar couplings, the scalar couplings could be comparable in size to the dipolar coupling and should not be neglected. The scalar couplings can be exploited to determine the signs of the dipolar couplings, as shown in chapter 5 .

\section{References}

(1) Sakurai, J. J. Modern Quantum Mechanics; Addison-Wesley Publishing Company, Inc., 1985. 
(2) Schmidt-Rohr, K.; Spiess, H. W. Multidimensional Solid-State NMR and Polymers; Academic Press Inc., San Diego, 1994.

(3) Harris, R. K. Nuclear Magnetic Resonance Spectroscopy; Longman Scientic \& Technical, Harlow, England, 1986.

(4) Ernst, R. R.; Bodenhausen, G.; Wokaun, A. Principles of Nuclear Magnetic Resonance in One and Two Dimensions; Clarendon Press, Oxford, 1987.

(5) Jeener, J.; Meier, B. H.; Bachmann, P.; Ernst, R. R. J. Chem. Phys. 1979, 71, 4546.

(6) Hagemeyer, A.; Schmidt-Rohr, K.; Spiess, H. W. Adv. Magn. Reson. 1989, 13, 85.

(7) States, D. J.; Haberkorn, R. A.; Ruben, D. J. J. Magn. Reson. 1982, 48, 286.

(8) Haeberlen, U. High Resolution NMR in Solids Selective Averaging; Academic Press, 1976.

(9) Mehring, M. High Resolution NMR in Solids; Springer-Verlag, New York, 1983.

(10) Abragam, A. Principles of Nuclear Magnetism; Clarendon Press, Oxford, 1961.

(11) Pake, G. E. J. Chem. Phys. 1948, 16, 327.

(12) Maricq, M. M.; Waugh, J. S. J. Chem. Phys. 1979, 70, 3300.

(13) Dixon, W. T. J. Chem. Phys. 1982, 77, 1800.

(14) Mansfield, P. J. Phys. Chem. 1971, 4, 1444.

(15) Rhim, W.-K.; Elleman, D. D.; Vaughan, R. W. J. Chem. Phys. 1973, 59, 1740.

(16) Pines, A.; Gibby, M. G.; Waugh, J. S. J. Chem. Phys. 1973, 59, 569.

(17) Hartmann, S. R.; Hahn, E. L. Phys. Rev. 1962, 128, 2042.

(18) Weitekamp, D. P.; Garbow, J. R.; Pines, A. J. Chem. Phys. 1982, 77, 2870.

(19) Caravatti, P.; Bodenhausen, G.; Ernst, R. R. Chem. Phys. Lett. 1982, 89, 363.

(20) Nakai, T.; Terao, T. Magn. Reson. Chem. 1992, 30, 42.

(21) Schmidt-Rohr, K.; Nanz, D.; Emsley, L.; Pines, A. J. Phys. Chem 1994, 98, 6668 . 
(22) Waugh, J. S. Proc. Natl. Acad. Sci. USA 1976, 73, 1394.

(23) Hester, R. K.; Ackermann, J. L.; Neff, B. L.; Waugh, J. S. Phys. Rev. Lett. 1976, 36, 1081.

(24) Opella, S. J.; Waugh, J. S. J. Chem. Phys. 1977, 66, 4919. 


\section{CHAPTER 3}

\section{PROPERTIES OF LIQUID-CRYSTALLINE PHOSPHOLIPIDS}

\subsection{Biological Importance}

Lipids are the basic structural component of biological membranes, which compartmentalize the fluid biological phases of most organisms. In prokaryotic and eukaryotic cells, a plasma membrane made up of a diacyl lipid bilayer separates the intracellular space from the extracellular environments. Subcellular organelles in eukaryotic cells such as the nucleus are also bound by lipid membranes. Lipid membranes protect the interior of the cells and organelles by providing a chemically selective permeability barrier. They also participate in energy and signal transductions of the organism. ${ }^{1-3}$

Phospholipids are the predominant lipid species in eukaryotic membranes. As such, the microscopic molecular structure and dynamics, and the "mesoscopic" distribution and organization of phospholipids strongly influence membrane functions. This can be understood on three levels. First, the oriented liquid-crystalline phospholipids provide a fluid environment in which other membrane cosurfactants such as proteins and polysaccharides function. For example, while the lipid bilayer itself is impermeable to polar substances, specific proteins imbedded in the bilayer can transport ions and metabolites across the membrane by forming channels or pumps through the lipid matrix. Some membrane proteins participate in energy transduction processes by undergoing lightor chemical-induced conformational changes in the phospholipid bilayer. The proper functioning of these proteins requires the surrounding lipid molecules to be highly mobile. This mobility can be provided by the acyl chains of the phospholipids. In addition to interacting with macromolecules, phospholipids also interact with small organic molecules such as sterols and alcohols, which are either intrinsic or transported to the membranes. Depending on their locations in the bilayer and their polarities, these small molecules can 
alter the membrane fluidity and permeability, thus adapting the mechanical properties of the membranes to various functions. ${ }^{4}$ A common example is the lipid-ethanol interaction, which induces visible physiological changes in the human body. ${ }^{5}$

Phospholipids not only provide a chemically inert and passive structure to the membranes, but also perform mechanical and chemical functions. For instance, the membrane-cytoskeleton complex generates forces that propel motile phagocytic organisms such as macrophages. The sphingomyelin lipids, present at high concentrations in the myelin sheath surrounding neural axons, not only protect the axons but also facilitate the transmission of neural impulses within the central nervous system.

The phospholipid compositions and lipid-protein ratios vary widely among membranes of different cells and of different species. But at the same time they are highly constant within each type of membranes. One example of this composition stability can be seen in the human neural cells, which contain a large amount and variety of glycosphingolipids such as cerebrosides, gangliosides and sphingomyelin. Although the functional roles of these glycosphingolipids are still largely unknown, the amounts of these sphingolipids are under tight genetic controls, and their deviations cause fatal diseases such as the Tay-Sachs disease and the Niemann-Pick disease. Another example is the human erythrocyte membrane, which contains several hundred lipids and proteins that are highlypreserved. Based on such empirical evidence, it can be concluded that the chemical and conformational specificity of phospholipids are strongly related to the functional diversity of the biological membranes. In fact, considering the protein-lipid coexistence in the membranes, one may even postulate that nature has selected different phospholipids in order to bring specific proteins together to form distinct functional units in the membranes. To understand these significant biochemical observations about phospholipids, it is important to determine the dynamic structure of the lipid molecules beyond the simple headgroup/acyl chain picture. 


\subsection{Chemical Structure}

All lipids are derivatives of fatty acids, which are carboxylic acids with a hydrocarbon chain of varying lengths. The lipid hydrocarbon chains are highly reduced and usually have even numbers ( 4 to 36 ) of carbon atoms. Fatty acids are named according to the lengths and degrees of unsaturation of the hydrocarbon chains (Table 3.1). For instance, a saturated fatty acid with 14 carbons (14:0) is called myristic acid, while a 16carbon chain with one double bond (16:1) is called palmitoleic acid.

\begin{tabular}{cc}
\hline fatty acid chains & common names \\
\hline $12: 0$ & lauric acid \\
$14: 0$ & myristic acid \\
$16: 0$ & palmitic acid \\
$18: 0$ & stearic acid \\
$20: 0$ & arachidic acid \\
$16: 1\left(\Delta^{9}\right)$ & palmitoleic acid \\
$18: 1\left(\Delta^{9}\right)$ & oleic acid \\
$18: 2\left(\Delta^{9,12}\right)$ & $\alpha$-linoleic acid
\end{tabular}

Table 3.1 Some naturally occurring fatty acids.

The simplest lipids are triacylglycerides, which consist of three fatty acids joined to a glycerol molecule through ester linkages. Triacylglycerides store energies in the eukaryotic cells and usually exist in a oil phase separate from the aqueous cytosol. ${ }^{3}$

Membrane phospholipids are composed of two fatty acid chains joined to a polar headgroup by a glycerol moiety. The headgroup is attached to the glycerol backbone through a phosphodiester bond while the two acyl chains are connected to it via ester linkages. In addition to these glycerophospholipids, two other types of structural lipids are found in biological membranes. Sphingolipids have a sphingosine (rather than a glycerol) 


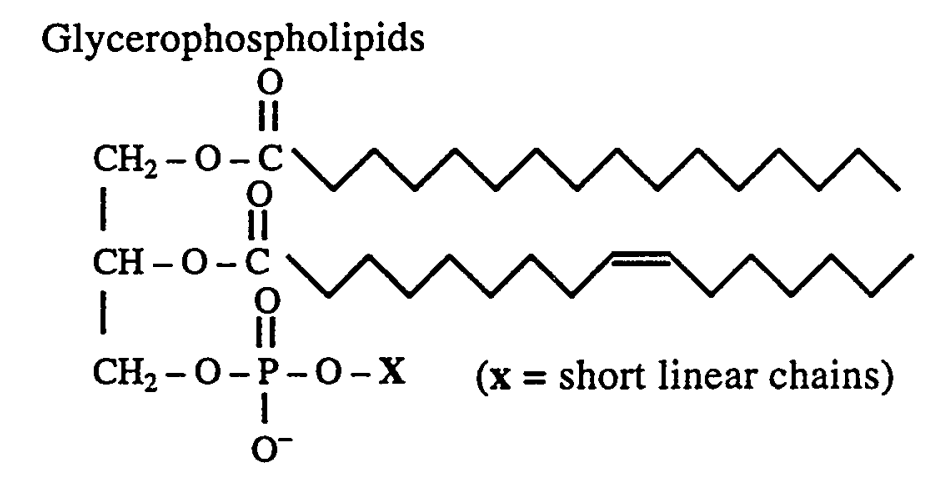

Sphingolipids

(including glycolipids)
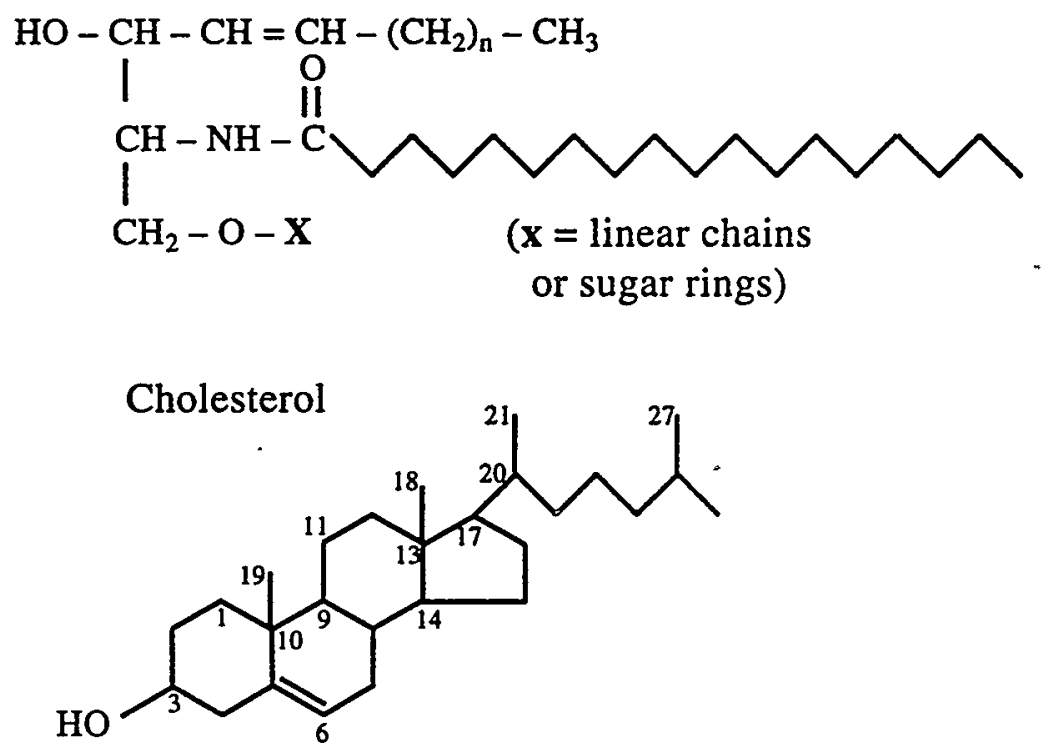

Figure 3.1 Three main types of membrane structural lipids.

backbone and one free acyl chain (instead of two), as the sphingosine moiety already possesses a long hydrocarbon chain. Sphingolipids with sugar residues in their headgroups are sometimes called glycolipids. They play important roles in the biological recognition at the cell surface. Sphingolipids such as the sphingomyelins and cerebrosides are of particular medical interest, due to their large concentrations in neural tissues and the human brain. The third type of membrane structural lipids is the sterols, of which cholesterol is a well-known example. Sterols differ from the glycerophospholipids and 
sphingolipids in that they have a rigid hydrophobic moiety made up of four fused hydrocarbon rings. The structures of these three types of membrane lipids are represented in Figure 3.1.

Because membrane lipids contain both a hydrophobic and a hydrophilic moiety, they are amphipathic (or amphiphilic) molecules. This amphipathicity has important consequences in the organization of the phospholipid molecules in the membranes.

Phospholipids are named according to the chemical constitutions of their headgroups and acyl chains (Table 3.2). Two common phospholipids are phosphatidylcholine ( $\mathrm{PC}$, also called lecithin), which has a choline headgroup $-\mathrm{CH}_{2}-\mathrm{CH}_{2}-\mathrm{N}^{+}\left(\mathrm{CH}_{3}\right)_{3}$, and phosphatidylethanolamine (PE), which has a $-\mathrm{CH}_{2}-\mathrm{CH}_{2}-\mathrm{NH}_{3}{ }^{+}$headgroup. Both headgroups are zwitterionic due to the +1 charge on the nitrogen atom and the -1 charge on the phosphate group. Other phospholipids such as phosphatidylserine have negatively charged headgroups. The electric charge of the headgroup influences the conformation and thus the functions of the specific phospholipid in the membranes.

\begin{tabular}{|c|c|c|}
\hline headgroup $(\mathrm{X})$ & name & charge \\
\hline \multicolumn{3}{|l|}{ glycerophospholipids } \\
\hline $\mathrm{CH}_{2}-\mathrm{CH}_{2}-\mathrm{N}^{+}\left(\mathrm{CH}_{3}\right)_{3}$ & phosphatidylcholine & 0 \\
\hline $\mathrm{CH}_{2}-\mathrm{CH}_{2}-\mathrm{NH}_{3}{ }^{+}$ & phosphatidylethanolamine & 0 \\
\hline $\mathrm{CH}_{2}-\mathrm{CH}\left(\mathrm{COO}^{-}\right)-\mathrm{NH}_{3}{ }^{+}$ & phosphatidylserine & -1 \\
\hline $\mathrm{CH}_{2}-\mathrm{CH}(\mathrm{OH})-\mathrm{CH}_{2} \mathrm{OH}$ & phosphatidylglycerol & -1 \\
\hline $\mathrm{CH}-(\mathrm{CHOH})_{5}$ & phosphatidylinositol & -1 \\
\hline \multicolumn{3}{|l|}{ sphingolipids } \\
\hline$-\mathrm{PO}_{3}{ }^{-}-\mathrm{CH}_{2}-\mathrm{CH}_{2}-\mathrm{N}^{+}\left(\mathrm{CH}_{3}\right)_{3}$ & sphingomyelin & 0 \\
\hline$-\mathrm{H}$ & ceramide & 0 \\
\hline$-\mathrm{CH}-(\mathrm{CHOH})_{3}-\mathrm{CH}\left(\mathrm{CH}_{2} \mathrm{OH}\right)-\mathrm{O}$ & glucosylcerebroside & 0 \\
\hline
\end{tabular}

Table 3.2 Headgroups of glycerophospholipids and sphingolipids. 


\subsection{Organized Assemblies}

Due to their amphipathic nature, phospholipids spontaneously aggregate into a separate phase when exposed to the aqueous environment. The hydrophobic chains are sequestered from the water molecules while the hydrophilic headgroups reside at the lipidwater interface. This self-assembly process is driven thermodynamically by the hydrophobic interactions, which tend to minimize the contact between the hydrophobic acyl chains and the polar water molecules. Once formed, the phospholipid aggregates are reinforced by noncovalent interactions such as the van der Waals interaction between the acyl chains.

Phospholipids can assemble into different aggregate structures, depending on the molecular shapes and the method of sample preparation. The primary organizational motif of phospholipids is the lamellar bilayer. The acyl chains in one monolayer face those in the other toward the center of the bilayer, whereas the headgroups point toward the bilayer surfaces in contact with water (Figure 3.2a). The bilayer is stabilized when the number of hydrophobic chains exposed to the aqueous environment is minimized. In actual biological systems, the lipid bilayers are almost invariably closed into spherical structures called vesicles (Figure 3.2b). Vesicles contain the cytosol and subcellular organelles in the case of plasma membranes and water molecules in the case of synthetic phospholipids. Multilamellar bilayer vesicles are often called liposomes (Figure 3.2c). The concentric bilayer shells in the liposomes are separated by aqueous layers. The liposomes and unilamellar vesicles have diameters of $30 \mathrm{~nm}-30 \mu \mathrm{m}$. In contrast to these large bilayer structures, micelles are small globules ( $<200 \AA$ in diameter) of a monolayer of lipids (Figure 3.2d). Depending on the polarity of the solvents surrounding them, micelles can have normal or inverted structures, corresponding to the water molecules and the polar headgroups being located outside or in the center of the globules. The small sizes of the micelles enable them to undergo isotropic rotational diffusions at rates faster than the 
spectroscopic NMR time scales, so that isotropically-averaged NMR spectra are obtained for this aggregate. In comparison, the larger lipid vesicles and liposomes tumble slowly on the NMR time scales, thus their NMR spectra are sensitive to local director fluctuations, and inhomogeneously broadened powder spectra are observed.

In addition to these common aggregate structures, phospholipids also form other supramolecular structures such as the hexagonal structure, the cubic and the rhombic phases. In the hexagonal phase, phospholipid tubes with diameters of $20-30 \mathrm{~nm}$ are distributed in a hexagonal array. A monolayer of phospholipids is oriented radially in the planes perpendicular to the cylindrical axis of the tube (Figure 3.2e).

Hydrated phospholipids can be prepared by mixing equal weight amounts of water and dry phospholipid powders, then subjecting the mixture to several freeze-thawing cycles to ensure uniform mixing. At this lipid-water molar ratio, the hydrated samples mostly form multibilayer liposomes. ${ }^{2} \mathrm{H}$ NMR has established convincingly that the synthetic phospholipid multibilayers have the same structural and dynamical properties as natural membranes. ${ }^{6}$ Therefore they can be used as model systems for elucidating the conformation and dynamics of phospholipids in biomembranes.

A large amount of evidence has established that phospholipids in the membranes are in constant motion. ${ }^{7}$ The predominant motional mode of phospholipids is uniaxial rotational diffusion - also called axial rotation or cylindrical symmetry - around the normal to the bilayer plane, with correlation times less than $10^{-7}$ seconds. The motional axis is called the director of the bilayer. However, the overall motion of the phospholipids around the director is not purely uniaxial rotation: at any instant in time a phospholipid molecule is unlikely to be parallel to the director, nor is it rotating at a constant rate. Rather, it is fluctuating or "wobbling" statistically with a time-averaged orientation along the director. Since the rotational correlation times $\left(\tau_{c}<10^{-7} \mathrm{~s}\right)$ are much shorter than the modulation periods of most NMR interactions $\left(\sim 10^{-5} \mathrm{~s}\right)$, the NMR spectra of these fluid-like phospholipids are anisotropically averaged. At lower temperatures, the molecules become 
more rigid and gel-like: their rotational diffusion rates are slower $\left(\tau_{c}>10^{-5} \mathrm{~s}\right)$, and transgauche isomerizations occur less frequently in the chain segments.

a

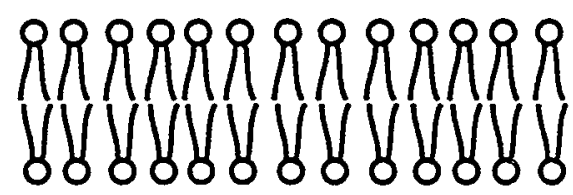

c

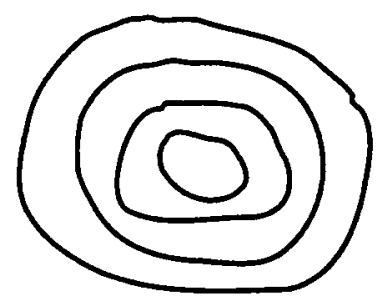

$\mathrm{b}$

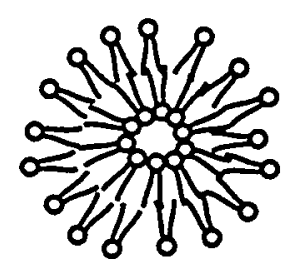

d

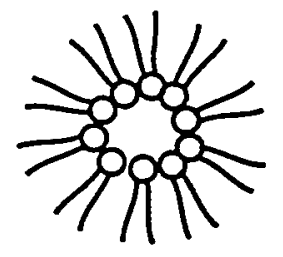

e

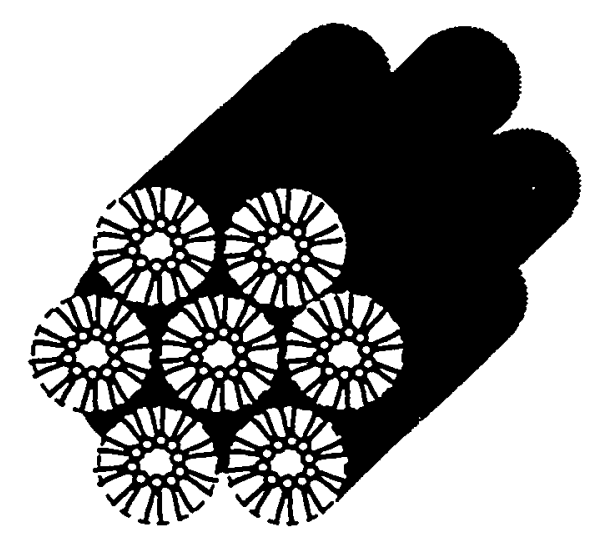

Figure 3.2 Organized phospholipid assemblies. (a) Bilayer. (b) Vesicle. (c) Liposome. (d) Micelle. (e) Hexagonal phase.

Another type of phospholipid motion is lateral diffusion across the bilayer plane. The rates of lateral diffusion differ between the low-temperature gel phases, which have hindered molecular translations, and the high-temperature fluid phase, in which the diffusion constants can be as large as $10^{-12} \mathrm{~m}^{2} / \mathrm{s}^{8,9}$ In other words, a phospholipid molecule in the fluid-phase diffuses an average of $2 \mu \mathrm{m}$ per second, which is a sufficient distance to cover the entire length of a bacterial cell membrane. This rapid lateral diffusion 
of the phospholipid molecules averages out intermolecular interactions, thus simplifying the NMR spectra.

Due to the uniaxial rotation and the lateral diffusion, membrane phospholipids exhibit partial orientational order (along the director) and positional order (random in the bilayer plane and ordered perpendicular to the plane), which are characteristic of liquid crystals. Specifically, bilayer phospholipids at physiological temperatures are characterized as in the liquid-crystalline $\mathrm{L}_{\alpha}$ phase. Similar to most liquid crystals, the phospholipids undergo a phase transition from the more-ordered gel phase to the $\mathrm{L}_{\alpha}$ phase at a characteristic temperature $T_{m}$.

At much lower rates, phospholipids also undergo transbilayer diffusion, or flipflops. Clearly, this motion requires the existence of non-bilayer lipid aggregates in the membranes, which is made possible by lipid polymorphism. ${ }^{10}$

In summary, phospholipids in biological membranes are oriented and mobile. Thus they are ideal solvents for membrane proteins and carbohydrates. The view of the biological membrane as an ever-changing entity, with proteins solvated in a sea of partially ordered lipids, is called the fluid mosaic model. ${ }^{7}$

\subsection{Physical Techniques for Studying Phospholipids}

A wide range of topics exists in the membrane lipid research. On the biological side, much effort has been made to understand the thermodynamic behavior of phospholipids in terms of temperature-hydration phase diagrams. ${ }^{11}$ Differential scanning calorimetry ${ }^{12,13}$ and electron microscopies are often employed in these studies.

On the physical side, scattering techniques have proved to be highly informative of the molecular structures of phospholipids. One of the clearest structural results was obtained by neutron scattering experiments conducted on phosphatidylcholine. By selective deuteration of the protons in various $\mathrm{CH}_{2}$ groups, high-sensitivity scattering density profiles were obtained. From these it was found that the headgroup of lecithin 
bends at the phosphate junction from the glycerol backbone and acyl chains. ${ }^{14}$ Since the glycerol backbone and the acyl chains are approximately parallel to the bilayer normal, as required by the bilayer asymmetry, such a bend causes the headgroup $\mathrm{O}-\mathrm{C} \alpha-\mathrm{C} \beta-\mathrm{N}$ backbone to be nearly parallel to the bilayer plane. The headgroup bend has since been found to be a ubiquitous feature of many phospholipids. According to molecular dynamics simulations, the orientation of the headgroup $\mathrm{P}^{-}-\mathrm{N}^{+}$dipole is titled by $10^{\circ}-30^{\circ}$ from the bilayer plane, with the positive end of the dipole reaching into the bilayer interior. ${ }^{15,16}$ The tilt angle is also influenced by the chemical constitution of the headgroup.

The phospholipid structure derived by neutron scattering has inherently low resolution due to the one-dimensional nature of the technique. In comparison, singlecrystal X-ray scattering of phospholipids at low hydration levels provides an atomic-scale electron density map of the molecules, but at the expense of observing the lipids in a biologically irrelevant state. The X-ray crystal structure of dimyristoylphosphatidylcholine $^{17}$ with $5 \%$ w/w hydration exhibits two conformers with different headgroup orientations. In conformation A, the headgroup points away from the glycerol moiety and the acyl chains, while in conformation $B$ the headgroup is folded back, making the molecular structure more compact.

The applicability of the crystal structure to the $L_{\alpha}$-phase phospholipids must be tested experimentally, since the conformational similarity between the crystalline and the liquid-crystalline phases is limited by crystal packing effects. For instance, the phospholipid acyl chains are known to be mostly trans in the gel and crystalline phases but have a higher gauche occurrence in the liquid-crystalline phase.

\subsection{Computer Simulations of Phospholipid Conformation and Dynamics}

In addition to the experimental studies, molecular dynamics simulations and $a b$ initio calculations have been carried out to study the dynamic conformations of phospholipids, especially lecithin. ${ }^{18-20}$ Generally, molecular dynamics simulations 
suggest very flexible structures, with a large conformational range for various torsion angles, including those in the headgroup and glycerol regions. ${ }^{15,16,21}$ This is not entirely consistent with the ${ }^{2} \mathrm{H}$ NMR results, which indicated that exchange of the headgroup between a pair of conformations would be sufficient to account for the ${ }^{2} \mathrm{H}$ quadrupolar splittings in these segments. However, the structural flexibility claimed by molecular dynamics simulations is possible, at least in a single-conformation model, if one considers that most bonds in lecithin are saturated and that no rings are present to make the structure more rigid. It seems that no clear consensus has been reached so far about the degrees of freedom of the phosphocholine headgroup. Given the theoretically predicted structural flexibility, one might question whether it is meaningful to approximate the liquid-crystalline phosphocholine by a single conformational model. The NMR experiments performed here (chapters 5 and 6) shed more light on this question.

\subsection{NMR Techniques for Studying Phospholipids}

Although non-NMR spectroscopic techniques such as infrared spectroscopy have been exploited to study the phase behavior of phospholipids, most structural information on phospholipids has been obtained by NMR spectroscopy. In the following, a brief overview of the results from various NMR techniques is given.

\section{${ }^{2} H$ Quadrupolar Couplings}

As the most widely-used NMR probe of phospholipid structure, the motionallyaveraged ${ }^{2} \mathrm{H}$ quadrupolar coupling provides information on the average orientation of a $\mathrm{C}-$ ${ }^{2} \mathrm{H}$ bond with respect to the director. ${ }^{21-25}$ By selective deuteration along the lipid chains, an order parameter profile was obtained as a function of the molecular segments. The profile shows a generally decreasing trend of the order parameter values toward the chain end, suggesting increasing disorder of the chain toward the bilayer center. This order profile resembles the mobility gradient determined from ${ }^{2} \mathrm{H}$ and ${ }^{13} \mathrm{C}$ relaxation studies. ${ }^{6}$ 
In the headgroup and the glycerol regions, the two ${ }^{2} \mathrm{H}$ quadrupolar couplings in each methylene group were found to be nearly degenerate. The degeneracy persists even when the coupling strengths are drastically changed by the incorporation of electric charges into the bilayers. ${ }^{26}$ To explain this degeneracy, it has been proposed that the phospholipid headgroup undergoes fast exchange between two mirror-symmetric conformations such that the geminal protons effectively interchange. ${ }^{23,27}$ The enantiomeric conformations were obtained by inverting the signs of all torsion angles in the glycerol and the headgroup regions. This exchange model is apparently consistent with the $\mathrm{X}$-ray crystal structure, which shows two molecules with distinct headgroup orientations in a unit cell.

${ }^{2} \mathrm{H}$ NMR has the drawback that it can only measure the absolute values, and not the signs, of the quadrupolar couplings. If the magnitude of the reduction factor between the averaged and the rigid-lattice ${ }^{2} \mathrm{H}$ quadrupolar splittings is smaller than $1 / 2$, which is the case for all sites in phosphocholine, then due to $-1 / 2 \leq P_{2}\left(\cos \theta_{C H}\right) \leq 1$, two orientations of the $\mathrm{C}-2 \mathrm{H}$ bonds are possible, and a distinction between them can not be made. This ambiguity can be eliminated by exploiting ${ }^{13} \mathrm{C}-1 \mathrm{H}$ dipolar couplings, as presented in chapter 5 .

\section{${ }^{31}$ P Chemical Shifts}

The $100 \%$ natural abundance of the ${ }^{31} \mathrm{P}$ spin and the large anisotropies of $31 \mathrm{P}$ chemical shift make ${ }^{31} \mathrm{P}$ NMR a convenient and sensitive probe of the electronic environment and orientation of the phosphate group. The lineshapes and linewidths of ${ }^{31} \mathrm{P}$ chemical shift spectra depend on several factors, including the average orientation of the phosphate with the bilayer director, the geometry of the phospholipid assembly, and the mechanisms and rates of motions of the phosphate moiety. The extraction of orientational information from averaged ${ }^{31} \mathrm{P}$ CSAs has benefited from the determination of the ${ }^{31} \mathrm{P}$ chemical shift tensor orientations in model phosphate compounds. ${ }^{28,29}$ Since the ${ }^{31} \mathrm{P}$ chemical shift tensor is not axially symmetric, two motional averaging parameters, the 
segmental order parameters, are necessary for obtaining quantitative orientational information. Thus, compared to the uniaxial ${ }^{2} \mathrm{H}$ quadrupolar coupling, the ${ }^{31} \mathrm{P}$ chemical shift is more difficult to interpret. On the other hand, the $31 \mathrm{P}$ chemical shift is uniquely sensitive to lipid polymorphism. ${ }^{10}$ For example, the ${ }^{31} \mathrm{P}$ spectrum of $\mathrm{PE}$ in the invertedhexagonal phase $\left(\mathrm{H}_{\mathrm{II}}\right)$ exhibits half the anisotropy as that of the bilayer $\mathrm{PE}$, and an opposite sign of anisotropy. ${ }^{30}$ Interestingly, the chemical structure of the lipid headgroup does not affect the ${ }^{31} \mathrm{P}$ spectra to any significant degree: different phospholipids with the same aggregate structure exhibit similar ${ }^{31} \mathrm{P}$ lineshapes and CSAs.

${ }^{31} \mathrm{P}$ NMR provides information on the rates of phosphate motion in the intermediate and slow regimes. The faster the motion, the smaller the anisotropy. In the fast motional limit, the ${ }^{31} \mathrm{P}$ chemical shift spectra of bilayer phospholipids are uniaxial with anisotropies of 40-50 ppm. The motional mechanism also influences the ${ }^{31} \mathrm{P}$ NMR lineshapes. ${ }^{9,32}$ Different powder lineshapes have been calculated for Brownian motion, two-site jump, and uniaxial rotation around the bilayer. ${ }^{31}$

\section{${ }^{13}$ C Chemical Shifts}

The isotropic ${ }^{13} \mathrm{C}$ chemical shifts are important for achieving site resolution in $2 \mathrm{D}$ NMR spectra. ${ }^{33}$ The anisotropic ${ }^{13} \mathrm{C}$ chemical shifts and their variations indicate the conformations of various phases. ${ }^{34,35}$ More quantitative structural information from the chemical shift can be extracted if the chemical shift tensor orientation in the molecular segment is known. For the headgroup and glycerol segments, the ${ }^{13} \mathrm{C}$ tensor orientations are still to be determined, whereas for the acyl chain segments the ${ }^{13} \mathrm{C}$ shift tensors can be assumed to be reasonably similar to those found in olefinic polymers. Two-dimensional NMR correlation techniques have been applied previously to unoriented phospholipids to obtain the site-resolved ${ }^{13} \mathrm{C} \mathrm{CSAs} .{ }^{36}$ These results were corroborated by experiments on oriented samples prepared in magnetically orientable detergent molecules. ${ }^{37}$ 
${ }^{1} H$ NMR

Protons in rigid solids form a multiple-spin system, thus the ${ }^{1} \mathrm{H}$ NMR spectra of normal solids are homogeneously broadened. This makes quantitative spectral analysis all but impossible. In contrast, $\mathrm{L}_{\alpha}$-phospholipids give rise to inhomogeneous ${ }^{1} \mathrm{H}$ spectra as a result of the fast anisotropic motions of the molecules. Specifically, intermolecular ${ }^{1} \mathrm{H}-1 \mathrm{H}$ dipolar couplings are averaged out by the lateral diffusion while intramolecular ${ }^{1} \mathrm{H}-{ }^{1} \mathrm{H}$ dipolar couplings are partially averaged by the uniaxial rotational diffusion. Furthermore, under magic-angle spinning, the multiple ${ }^{1} \mathrm{H}$ spin system is inhomogeneous, since the uniform orientation dependence of the motionally-averaged spin interactions makes the ${ }^{1} \mathrm{H}$ homonuclear Hamiltonian commute at different times. As a result, the ${ }^{1} \mathrm{H}$ MAS spectrum of $\mathrm{L}_{\alpha}$-phospholipids exhibits a centerband and sidebands for each ${ }^{1} \mathrm{H}$ site, analogous to the rare-spin spectrum of a rotating solid. ${ }^{38}$ Comparisons between the static and the MAS ${ }^{1} \mathrm{H}$ spectra help understand ${ }^{1} \mathrm{H}$ spin diffusion in the lipid bilayer and the effects of sonication on the bilayer structure. Furthermore, the high-resolution ${ }^{1} \mathrm{H}$ spectra enable the assignment of inequivalent ${ }^{1} \mathrm{H}$ sites in the phospholipids. ${ }^{39}$

In principle, NMR can provide detailed information on the segmental orientation and dynamics of phospholipids in the $\mathrm{L}_{\alpha}$ phase. In practice, the amount of NMR data available so far does not permit rigorous quantification of such information. Thus more experimental NMR couplings need to be measured. There is a more fundamental problem with applying NMR techniques to anisotropic systems, namely, that the motionallyaveraged NMR spectra cannot distinguish effects due to segmental orientations from effects due to motional rates and amplitudes. Due to the liquid-crystalline nature of the phospholipid bilayers, the molecular conformations of the phospholipid molecules cannot be characterized simply by distances and orientational parameters. The only available theory able to convert the motionally-averaged NMR couplings to structural information is the segmental order tensor formalism. It yields information on segmental orientations and 
thus conformational probabilities of the molecules. Before we present the results of our NMR measurements in phospholipids and liquid crystals, we first introduce this order tensor formalism in the following chapter.

\section{References}

(1) Stryer, L. Biochemistry; 4th ed. ed.; W.H. Freeman and Company, New York, 1995, pp 1064.

(2) Petty, H. R. Molecular biology of membranes: structure and function; Plenum, New York, 1993.

(3) Lehninger, A. L.; Nelson, D. L.; Cox, M. M. Principles of Biochemistry; Worth Publishers, New York, 1993.

(4) Bloom, M.; Evans, E.; Mouritsen, O. G. Q. Rev. Biophys. 1991, 24, 293.

(5) Barry, J. A.; Gawrisch, K. Biochemistry 1994, 33, 8082.

(6) Seelig, J.; Seelig, A. Q. Rev. of Biophys. 1980, 13, 19.

(7) Singer, S. J.; Nicolson, G. L. Science 1972, 175, 720.

(8) Seelig, J. Biochim. Biophys. Acta 1978, 515, 105.

(9) Hemminga, M. A.; Cullis, P. R. J. Magn. Reson. 1982, 47, 307.

(10) Cullis, P. R.; Kruijff, B. D. Biochim. Biophys. Acta 1979, 559, 399.

(11) Silver, B. L. The physical chemistry of membranes: an introduction to the structure and dynamics of biological membranes; Solomon Press, Jamaica, N.Y., 1985.

(12) Hui, S. W.; Stewart, T. P.; Yeagle, P. L. Biochim. Biophys. Acta 1980, 601, 271.

(13) Maulik, P. R.; Sripada, P. K.; Shipley, G. G. Biochim. Biophys. Acta 1991, 1062, 211.

(14) Büldt, G.; Gally, H. U.; Seelig, J.; Zaccai, G. Nature 1978, 271, 182.

(15) Konstant, P. H.; Pearce, L. L.; Harvey, S. C. Biophys. J. 1994, 67, 713. 
(16) Stouch, T. R. Mol. Simul. 1993, 10, 335.

(17) Pearson, R. H.; Pascher, I. Nature 1979, 281, 499.

(18) Strenk, L. M.; Westerman, P. W.; Doane, J. W. Biophys. J. 1985, 48, 765.

(19) Pastor, R. W.; Venable, R. M.; Karplus, M. Proc. Natl. Acad. Sci. USA 1991, 88,892 .

(20) Wiener, M. C.; White, S. H. Biophys. J. 1992, 61, 434.

(21) Skarjune, R.; Oldfield, E. Biochemistry 1979, 18, 5903.

(22) Seelig, A.; Seelig, J. Biochim. Biophys. Acta 1975, 406, 1.

(23) Seelig, J.; Gally, H.-U.; Wohlgemuth, R. Biochim. Biophys. Acta 1977, 467, 109.

(24) Seelig, J.; Macdonald, P. M.; Scherer, P. G. Biochemistry 1987, 26, 7535.

(25) Blume, A.; Rice, D. M.; Wittebort, R. J.; Griffin, R. G. Biochemistry 1982, 21, 6220.

(26) Scherer, P. G.; Seelig, J. Biochemistry 1989, 28, 7723.

(27) Gally, H.; Niederberger, W.; Seelig, J. Biochemistry 1975, 14, 3647.

(28) Kohler, S. J.; Klein, M. P. Biochemistry 1976, 15, 968.

(29) Hauser, H.; Radloff, C.; Ernst, R. R.; Sundell, S.; Pascher, I. J. Am. Chem. Soc. 1988, 110, 1054.

(30) Thayer, A. M.; Kohler, S. J. Biochemistry 1981, $20,6831$.

(31) Smith, I. C. P.; Ekiel, I. H. In Phosphorous-31 NMR: Principles and Applications;

I. C. Gorenstein, Ed.; 1984.

(32) Niederberger, W.; Seelig, J. J. Am. Chem. Soc. 1976, 98, 3704.

(33) Husted, C.; Montez, B.; Le, C.; Moscarello, M. A.; Oldfield, E. Magn. Res. Med 1993, 29, 168.

(34) Wittebort, R. J.; Blume, A.; Huang, T.; Gupta, S. K. D.; Griffin, R. G. Biochemistry 1982, 21, 3487. 
(35) Huang, T. H.; Lee, C. W. B.; Gupta, S. K. D.; Blume, A.; Griffin, R. G. Biochemistry 1993, 32, 13277.

(36) Braach-Maksvytis, V. L. B.; Cornell, B. A. Biophys. J. 1988, 53, 839.

(37) Sanders, C. R. Biophys. J. 1993, 64, 171.

(38) Forbes, J.; Husted, C.; Oldfield, E. J. Am. Chem. Soc. 1988, 110, 1059.

(39) Li, K.; Tihal, C. A.; Guo, M.; Stark, R. E. Biochemistry 1993, 32, 9926. 


\section{CHAPTER 4 \\ THEORY OF ORDER TENSOR FOR PHOSPHOLIPIDS AND LIQUID CRYSTALS}

\subsection{Effects of Liquid Crystallinity on Phospholipid NMR Spectra}

Under biological conditions, lipid membranes are liquid crystalline, with phospholipid molecules undergoing fast uniaxial motions around the normal of the bilayer. Such uniaxiality is "macroscopic", in the sense that the motional axis (the director) is a property of the mesophase structural unit (the bilayer) external to the molecules. If the lipid molecules also undergo rotation about a molecule-fixed rotational axis, then they are called "microscopically" uniaxial. Microscopically uniaxial molecules are usually rigid: the intramolecular segments have fixed orientations with respect to each other. Macroscopic uniaxiality is directly reflected in and proved by NMR lineshapes, while microscopic uniaxiality is more difficult to determine (since it requires uniaxial order tensors).

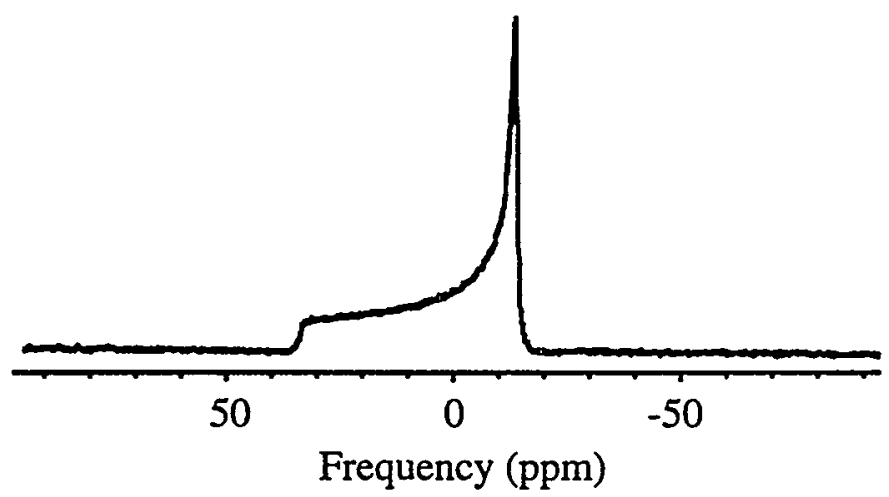

Figure 4.1 $\mathrm{L}_{\alpha}$-lecithin ${ }^{31} \mathrm{P}$ single-pulse chemical shift spectrum.

Hydrated phospholipids exhibit an isotropic distribution of bilayer orientations with respect to the magnetic field, provided no mechanical or chemical methods are used to induce additional orientations in the sample. Because of the random nature of the director 
orientations, the local NMR spin interactions give rise to inhomogeneously-broadened NMR spectra. For example, the ${ }^{31} \mathrm{P}$ chemical shift spectrum (Figure 4.1) of lecithin exhibits a typical $\eta=0$ lineshape and an anisotropy of $-47 \mathrm{ppm}$.

The isotropic distribution of the lipid bilayers with respect to the magnetic field must be distinguished from the anisotropy of the molecular motions with respect to the director. The former is a static property referenced to the laboratory frame, whose $z$-axis is defined as the Zeeman field direction (Figure 4.2). The latter is a dynamical property best described in a molecular frame, in which the director orientations are distributed in a restricted region on a unit sphere. The width of this distribution is reflected in the magnitudes of motionally-averaged NMR couplings.

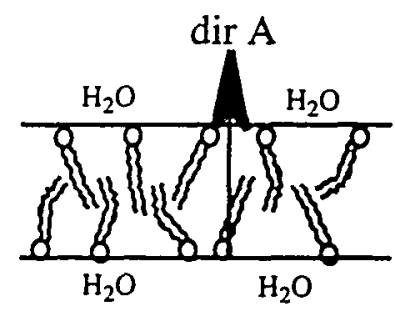

bilayer A

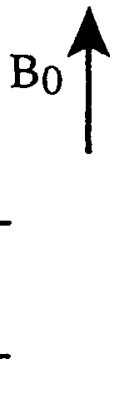

bilayer B

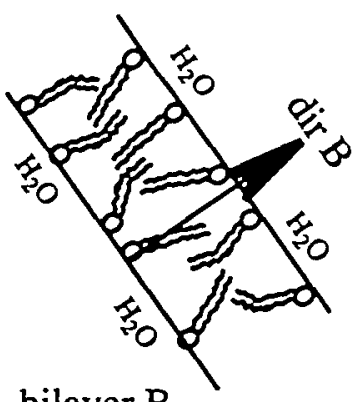

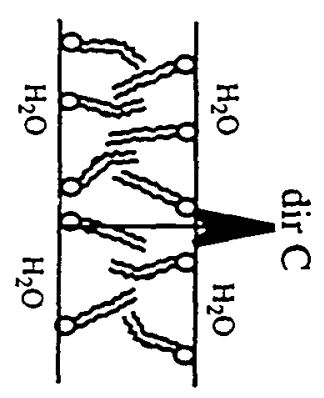

bilayer $\mathrm{C}$

Figure 4.2 Schematic of phospholipid bilayers. The random orientations of the bilayer directors with the $B_{0}$ field give rise to NMR "powder" spectra. The phospholipid molecules undergo uniaxial rotations with respect to the directors.

The fast uniaxial rotation of the phospholipid molecules has two consequences on the NMR spectra. First, it averages the NMR interaction tensors to be axially symmetric, with the unique axes parallel to the director. Thus all interaction tensors have the same orientation dependence with the magnetic field: they are scaled by $\frac{1}{2}\left(3 \cos ^{2} \theta_{d}-1\right)$, where $\theta_{d}$ is the angle between the director and $\vec{B}_{0}$. A consequence of this "parallelity" is the "magic-angle hole" in the ${ }^{1} \mathrm{H}$-cross-polarized ${ }^{31} \mathrm{P}$ spectrum (Figure 4.3). The vanishing intensity at the isotropic chemical shift results from the vanishing $\mathrm{P}-\mathrm{H}$ dipolar couplings in 
bilayers whose directors are inclined at the magic angle $\theta_{d}=54.7^{\circ}$ with respect to the magnetic field.

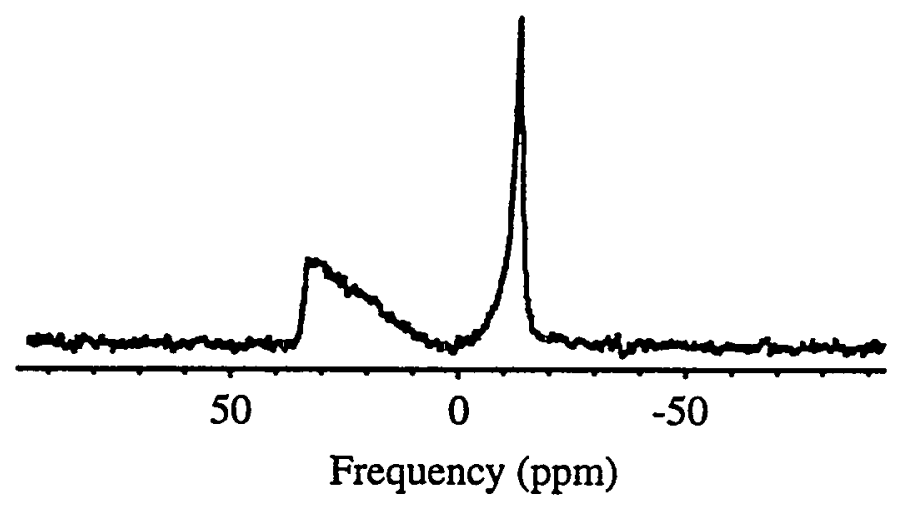

Figure 4.3 $\mathrm{L}_{\alpha}$-lecithin ${ }^{31} \mathrm{P}$ cross-polarized chemical shift spectrum.
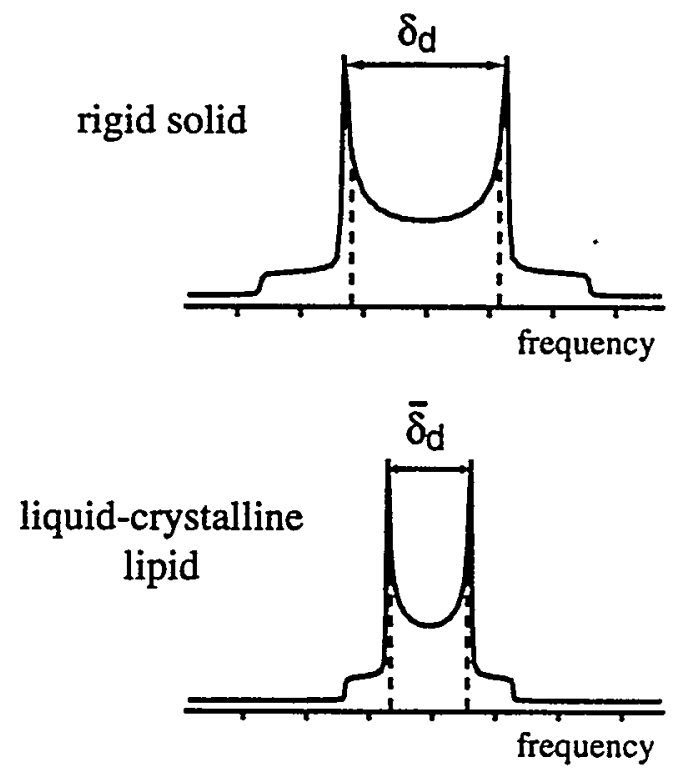

Figure 4.4 Dipolar spectra of a rigid solid and an anisotropically-mobile lipid. Dipolar couplings in the lipid are reduced by fast anisotropic motions.

The fast anisotrcpic motions of phospholipids also reduce the NMR couplings from their rigid-lattice values. For the heteronuclear dipolar interaction, the splitting between the two maxima of the Pake pattern is narrowed from the rigid-limit value $\delta_{d}$ to an averaged value $\bar{\delta}_{d}$ (Figure 4.4). For the chemical shift interaction, not only is the spectral width 
reduced, but also the lineshape changes from $\eta \neq 0$ to $\eta=0$. The degree of narrowing, $\bar{\delta} / \delta$, contains information on segmental conformation and dynamics, which can be summarized elegantly by the segmental order tensor.

\subsection{Derivation of Order Tensor}

Consider the effects of uniaxial molecular motion on the chemical shift interaction $\sigma .^{1}$ The fast motion averages the rigid-lattice shift tensor, defined in its PAS as

$$
\sigma^{\mathrm{PAS}}=\left(\begin{array}{lll}
\sigma_{x x} & & \\
& \sigma_{y y} & \\
& & \sigma_{z z}
\end{array}\right),
$$

to an axially symmetric tensor in the director frame,

$$
<\sigma^{D}>=\left(\begin{array}{lll}
\sigma_{\perp} & & \\
& \sigma_{\perp} & \\
& & \sigma_{\| \prime}
\end{array}\right),
$$

where the component $\sigma_{/ /}$is parallel to the director and $\sigma_{\perp}$ is perpendicular to it. To arrive at this averaged chemical shift tensor, the first step is to transform the original $\sigma$ from its PAS $\left(x_{P}, y_{P}, z_{P}\right)$ to the director frame $\mathrm{D}\left(x_{D}, y_{D}, z_{D}\right)$,

$$
\sigma^{\mathrm{D}}=R\left(\alpha_{p}, \beta_{p}, 0\right) \sigma^{\mathrm{PAS}} R^{-1}\left(\alpha_{p}, \beta_{p}, 0\right)
$$

The rotation matrix $R\left(\alpha_{p}, \beta_{p}, 0\right)$ is defined as

$$
R\left(\alpha_{p}, \beta_{p}, 0\right)=\left(\begin{array}{ccc}
\cos \alpha_{p} \cos \beta_{p} & \sin \alpha_{p} \cos \beta_{p} & -\sin \beta_{p} \\
-\sin \alpha_{p} & \cos \alpha_{p} & 0 \\
\cos \alpha_{p} \sin \beta_{p} & \sin \alpha_{p} \sin \beta_{p} & \cos \beta_{p}
\end{array}\right)
$$


The three Euler angles of rotation are defined as follows. ${ }^{2}$ A rotation of an angle $\alpha_{p}$ around the $z P$ axis brings the $\sigma^{\mathrm{PAS}}$ to an intermediate position, whose $y$-axis is the common axis of the $x_{P} y_{P}$ and the $x_{D} y_{D}$ plane. A second rotation of an angle $\beta_{p}$ about this intermediate $y$-axis aligns the $z P$ axis with $z_{D}$. Since the director frame is uniaxial with respect to the $z_{D}$ axis, the third angle $\gamma_{p}$ is zero. Note that the Euler angles $\alpha_{p}$ and $\beta_{p}$ defined as such coincide with the spherical polar angles $\phi$ and $\theta$, respectively.

The transformed chemical shift tensor must be averaged to account for the fast uniaxial rotation. This eliminates the off-diagonal terms in $\sigma^{D}$ and gives

$$
<\sigma^{D}>=\left(\begin{array}{lll}
\frac{3}{2} \sigma_{i s o}-\frac{1}{2} \sigma_{\| /} & & \\
& \frac{3}{2} \sigma_{i s o}-\frac{1}{2} \sigma_{\| l} & \\
& & \sigma_{/ /}
\end{array}\right) \text {, }
$$

where the parallel component $\sigma_{/ /}$is related to the principal values $\sigma_{i i}(i=x, y, z)$ according to

$$
\sigma_{/ /}=\left\langle\sin ^{2} \beta_{p} \cos ^{2} \alpha_{p}\right\rangle \cdot \sigma_{x x}+\left\langle\sin ^{2} \beta_{p} \sin ^{2} \alpha_{p}\right\rangle \cdot \sigma_{y y}+\left\langle\cos ^{2} \beta_{p}\right\rangle \cdot \sigma_{z z} .
$$

The isotropic chemical shift $\sigma_{i s o}$ is the trace of the principal values,

$$
\sigma_{i s o}=\frac{1}{3} \sum_{i} \sigma_{i i}
$$

The time-averaged trigonometric terms in equation (4.6) can be expressed concisely by a set of order parameters, defined as

$$
S_{i i}=\frac{1}{2}\left\langle 3 \cos ^{2} \Theta_{i}-1\right\rangle,
$$


where $\cos \Theta_{i}$ is the directional cosine of the angle $\Theta_{i}$ between the director and the $i$-axis of the chemical shift PAS. The directional cosines are related to the Euler angles $\left(\alpha_{p}, \beta_{p}, \gamma_{p}\right)$ by

$$
\cos \Theta_{x}=\sin \beta_{p} \cos \alpha_{p}, \quad \cos \Theta_{y}=\sin \beta_{p} \sin \alpha_{p}, \quad \cos \Theta_{z}=\cos \beta_{p}
$$

With these newly-introduced order parameters, the parallel component $\sigma_{/ /}$of $\left\langle\sigma^{D}>\right.$ and the averaged chemical shift anisotropy $\Delta \bar{\sigma}$ can be written as

$$
\sigma_{/ /}-\sigma_{i s o} \equiv \frac{2}{3} \Delta \bar{\sigma}=\frac{2}{3} \sum_{i} S_{i i} \sigma_{i i}
$$

Therefore, the averaged chemical shift anisotropy is directly related to the order parameters, which is shown later to summarize the motional averaging of the molecules. Generally, all averaged NMR anisotropies, such as the dipolar and the quadrupolar coupling, are related to the order parameters $S_{i i}$ by the same expression (4.10).

Although the motionally-averaged NMR couplings can be calculated from the interaction tensor PAS, it is often advantageous to start with a coordinate system that preserves the symmetry of the molecular segment. For uniaxial systems, an NMR interaction $\mathbf{T}$ can be transformed from any segment-fixed frame $\mathrm{M}\left(x_{M}, y_{M}, z_{M}\right)$ to the director frame $\mathrm{D}\left(x_{D}, y_{D}, z_{D}\right)$. This transformation is simplified with the help of a generalized order matrix $S$, called the Saupe matrix, 3,4

$$
S_{i j}=\frac{1}{2}\left\langle 3 \cos \Theta_{i} \cos \Theta_{j}-\delta_{i j}\right\rangle,
$$

where $\cos \Theta_{i}(i=x, y, z)$ is the directional cosine between the $i$-axis of the segment-fixed frame $M$ and the director. Since the measured NMR frequency is the $z$-component of the 
interaction tensor $\mathbf{T}$ in the laboratory frame $L\left(x_{L}, y_{L}, z_{L}\right)$, where $z_{L}$ is along the $\vec{B}_{0}$ direction, it can be obtained from two successive coordinate transformations,

$$
T^{M} \stackrel{\Theta_{i j}}{\longrightarrow} T^{D} \stackrel{\Phi_{i j}}{\longrightarrow} T^{L} .
$$

Here $\Theta_{i j}$ and $\Phi_{i j}$ specify the directional angles between the $i$-axis of the first coordinate and the $j$-axis of the second. The directional cosines are used instead of Euler angles for tensor rotations because they indicate the role of the order matrix explicitly.

Since $\mathrm{T}^{\mathrm{D}}$ is axially symmetric, the only relevant frequency in the transformation $\mathbf{T}^{\mathrm{M}} \rightarrow \mathbf{T}^{\mathrm{D}}$ is the frequency along the $z_{D}$ (director) axis,

$$
T_{z z}^{D}=\sum_{i, j} \cos \Theta_{i z} \cdot T_{i j}^{M} \cdot \cos \Theta_{j z}
$$

This frequency is averaged by the uniaxial rotational diffusion

to give

$$
\begin{aligned}
\left\langle T_{z z}^{D}\right\rangle & =\sum_{i, j}\left\langle\cos \Theta_{i z} \cos \Theta_{j z}\right\rangle T_{i, j}^{M} \\
& =\frac{2}{3} \sum_{i, j}\left\langle\frac{3}{2} \cos \Theta_{i z} \cos \Theta_{j z}-\frac{1}{2} \delta_{i, j}\right\rangle T_{i, j}^{M}+\frac{1}{3} \sum_{i, j} \delta_{i, j} T_{i, j}^{M}
\end{aligned}
$$

$$
\Rightarrow \bar{\delta} \equiv\left\langle T_{z z}^{D}\right\rangle-T_{i s o}=\frac{2}{3} \sum_{i, j} S_{i j}^{M} T_{i j}^{M}
$$

The above calculation is repeated to transform $T^{D}$ to the laboratory frame in order to obtain the observed NMR frequency $T_{z z}^{L}$,

$$
\begin{aligned}
T_{z z}^{L}-T_{i s o} & =\sum_{i} \cos \Phi_{i z} \cdot\left\langle T_{i i}^{D}-T_{i s o}\right\rangle \cdot \cos \Phi_{i z} \\
& =-\frac{1}{2} \bar{\delta}\left(\cos ^{2} \Phi_{x z}+\cos ^{2} \Phi_{y z}\right)+\bar{\delta} \cos ^{2} \Phi_{z z} \\
& =-\frac{1}{2} \bar{\delta}\left(1-\cos ^{2} \Phi_{z z}\right)+\bar{\delta} \cos ^{2} \Phi_{z z}
\end{aligned}
$$




$$
=\bar{\delta} \cdot \frac{1}{2}\left(3 \cos ^{2} \Phi_{z z}-1\right)
$$

Equation (4.14) expresses the NMR frequency dependence on the bilayer orientation, as the directional angle $\Phi_{2 z}$ coincides with the polar angle of the director in the laboratory frame. This equation is analogous to expression (2.60), which describes the powder averaging in polycrystalline solids, except that tensor $\mathrm{T}^{\mathrm{D}}$ is always axially symmetric $(\eta=0)$ due to the uniaxial motion. Therefore, the NMR spectra of phospholipids are fully determined if the averaged anisotropy parameters $\bar{\delta}$ are known. According to equation (4.13), $\bar{\delta}$ can be obtained from the scalar product of $\mathbf{T}$ and $\mathbf{S}$ expressed in a common segment-fixed frame. The calculation for $\bar{\delta}$ can be simplified if the segmental frame $M$ is the PAS of the NMR interaction $T$ or the PAS of $S$, or if $M$ incorporates the symmetry of the molecular segment. If both the orientation and the magnitude of the interaction tensor in a segment are known, as for the dipolar coupling between a pair of spins separated by a fixed distance, or for a chemical shift tensor whose PAS is determined experimentally, then the NMR frequencies are determined only by the $\mathbf{S}$ tensor. Therefore, the $\mathbf{S}$ tensor contains maximum information attainable by NMR about the motionally-averaged orientation of the segment with respect to the director. Once the $\mathbf{S}$ tensor is solved, any segment-fixed averaged NMR coupling can be predicted.

The order matrix is traceless and symmetric. Its trace is zero because

$$
\sum_{i} \cos \Theta_{i}=\sin ^{2} \theta \cos ^{2} \phi+\sin ^{2} \theta \sin ^{2} \phi+\cos ^{2} \theta=1
$$

which leads to

$$
\sum_{i} S_{i i}=\frac{3}{2} \sum_{i} \cos ^{2} \Theta_{i}-\frac{3}{2}=0
$$

Here $\theta$ and $\phi$ denote the polar angles of the director in a segment-fixed frame. The order matrix is also symmetric, i.e. $S_{i j}=S_{j i}$, because $\cos \Theta_{i} \cos \Theta_{j}=\cos \Theta_{j} \cos \Theta_{i}$. Therefore, a $\mathbf{S}$ 
matrix has five independent elements, and can always be diagonalized to produce its own principal axis system.

\subsection{Phenomenological Description of Order Tensor}

Further insight into the order tensor may be gained by introducing it alternatively in the context of liquid crystals. Liquid crystals are materials with partial positional and orientational orders, which make them an intermediate phase (mesophase) of matter between rigid crystalline solids and isotropic and mobile liquids. Depending on the types and degrees of order, liquid crystals have various phases such as the nematic, smectic and lamellar phases. The nematic phase, for example, exhibits orientational order along a spatial axis but no positional order, and often occurs in rod-shaped organic molecules. Due to this orientational order, NMR spectra of nematic liquid crystals exhibit sharp lines at anisotropic frequencies. These frequencies depend not only on the rigid-lattice NMR tensor parameters but also on the degree of orientational order.

Consider the orientation distribution of a nematic liquid crystal made up of rodshaped molecules. ${ }^{5}$ The orientation of the molecular cylindrical axis $\vec{a}$ can be defined by the spherical angles $\left(\theta_{l}, \phi_{l}\right)$ in the laboratory frame. The distribution of $\vec{a}$ around the nematic axis $\vec{n}$ can be described by a distribution function $f\left(\theta_{l}, \phi_{l}\right)$. Due to the phase uniaxiality around $\vec{n}, f\left(\theta_{l}, \phi_{l}\right)$ is independent of the azimuth angle $\phi_{l}$. Moreover, because the "up" and "down" directions along $\bar{n}$ are indistinguishable, the distribution is symmetric with respect to the plane normal to $\vec{n}$, i.e. $f\left(\theta_{l}\right)=f\left(\pi-\theta_{l}\right)$. Under these conditions, we seek a function whose numerical average by the distribution function $f\left(\theta_{l}\right)$ reflects the orientational order of the molecules around the nematic axis. One obvious choice is the lowest-order anisotropic spherical harmonic, $Y_{1,0} \propto \cos \theta$. However, its ensemble average,

$$
\left\langle\cos \theta_{l}\right\rangle=\int_{0}^{2 \pi} f\left(\theta_{l}\right) \cos \theta_{l} d\left(\cos \theta_{l}\right) \equiv 0,
$$


vanishes identically to zero regardless of the distribution $f\left(\theta_{l}\right)$. So $Y_{1,0}$ is unsuitable for representing the orientational order. Next, we try the second-order spherical harmonic $Y_{2,0} \propto \frac{1}{2}\left(3 \cos ^{2} \theta-1\right)$. It is averaged by $f\left(\theta_{l}\right)$ as

$$
\frac{1}{2}\left\langle 3 \cos ^{2} \theta_{l}-1\right\rangle=\int_{-1}^{0} d\left(\cos \theta_{l}\right) f\left(\theta_{l}\right) \frac{1}{2}\left(3 \cos ^{2} \theta_{l}-1\right)=\left\{\begin{array}{cc}
1 & \theta_{l}=0^{\circ} \\
-\frac{1}{2} & \theta_{l}=90^{\circ} \\
0 & \theta_{l}: \text { random }
\end{array}\right.
$$

The ensemble-averaged function $\left\langle Y_{2,0}\right\rangle$ takes on values between $-1 / 2$ and +1 . The value +1 corresponds to perfect alignment of the molecules with the nematic axis, whereas 0 indicates an isotropic distribution. Therefore, the representation of the orientational order by $\left\langle Y_{2,0}\right\rangle$ is consistent with common intuitions. We define this average parameter as the molecular order parameter $S_{\text {mol }}$

$$
S_{m o l}=\frac{1}{2}\left\langle 3 \cos ^{2} \theta_{l}-1\right\rangle
$$

The molecular order parameter $S_{m o l}$ is a simplification of the second-rank tensor $\mathbf{S}$ defined in equation (4.11). In fact, the $\mathbf{S}$ tensor could be derived analogously by considering the orientation distribution of arbitrarily-shaped molecules in a uniaxial phase.

\subsection{Relation Between Order Tensor and Order Parameter}

The importance and relevance of the order tensor $\mathbf{S}$ are expressed in relation (4.13) between $S$ and the motionally-averaged NMR anisotropy $\bar{\delta}$ :

$$
\bar{\delta}=\frac{2}{3} \sum_{i, j} S_{i j} T_{i j}
$$


where the interaction tensor $T_{i j}$ is expressed in the same segment-fixed frame as $S_{i j}$. The superscript $M$ indicating the frame is removed in the above equation, since any segmentfixed frame, including the NMR tensor PAS and the S PAS, can be used.

For axially symmetric NMR interactions such as two-spin dipolar coupling, equation (4.20) takes on various guises in the literature. In the following, two equivalent forms of the above relation are given. First, in terms of the polar coordinates $\left(\theta_{S}, \phi_{S}\right)$ of the unique axis of the interaction tensor $\mathbf{T}$ in the $\mathbf{S}$ tensor PAS,

$$
\bar{\delta}=\frac{\delta}{2}\left[S_{z z}^{P A S}\left(3 \cos ^{2} \theta_{s}-1\right)+\left(S_{x x}^{P A S}-S_{y y}^{P A S}\right) \sin ^{2} \theta_{s} \cos 2 \phi_{s}\right]
$$

Second, expressed in the $\mathbf{T}$ tensor PAS,

$$
\bar{\delta}=\delta \cdot S_{z z}^{T, P A S} .
$$

The first relation (4.21) is derived as follows. Consider the representation of the interaction $\mathbf{T}(\eta=0)$ in an arbitrary reference frame $R$. If $X_{\text {in }}$ indicates the directional angle between the $i$-axis of TPAS and the $n$-axis of the reference frame, then the diagonal elements of $\mathbf{T}^{\mathrm{R}}$ can be calculated in an analogous manner to equation (4.14) as

$$
\begin{aligned}
T_{n n}^{R} & =\sum_{i} \cos \mathrm{X}_{i n} T_{i i}^{P A S} \cos \mathrm{X}_{i n} \\
& =\dot{\delta} \cdot \frac{1}{2}\left(3 \cos ^{2} \mathrm{X}_{z n}-1\right)
\end{aligned}
$$

Again, due to the axial symmetry of $\mathbf{T}$, only the directional $\operatorname{cosines} \cos \mathrm{X}_{z n} \equiv \cos \gamma_{n}$ $(n=x, y, z)$ between the unique axis $z$ (e.g. the internuclear vector) and the $n$-axis of the reference frame are required for calculating $T_{n n}^{R}$. Therefore, $T_{n n}^{R}=\delta \cdot \frac{1}{2}\left(3 \cos ^{2} \gamma_{n}-1\right)$.

Specifically, the averaged NMR anisotropy is the scalar product of $\mathbf{S}$ and $\mathbf{T}$, which can be both expressed in the $\mathbf{S}$ tensor PAS. Applying equation (4.23), we obtain 


$$
\begin{aligned}
& \bar{\delta}=\frac{2}{3} \sum_{j, k} S_{j k} T_{j k}=\frac{2}{3} \sum_{j} S_{j j}^{P A S} T_{j j} \\
& =\frac{2}{3} \frac{\delta}{2} \sum_{j} S_{j j}^{P A S}\left(3 \cos ^{2} \gamma_{j}-1\right)=\delta \sum_{j} S_{j j}^{P A S} \cos ^{2} \gamma_{j} .
\end{aligned}
$$

Note that the directional angle $\gamma_{j}$ here specifies the angle between the unique axis of TPAS and the $j$-axis of the $S^{\text {PAS }}$. Equation (4.24) indicates that the order parameter of a vector, $\bar{\delta} / \delta$, is equal to the projection of the $\mathbf{S}$ matrix onto that vector. (Such a relation is generally true when transforming a second-rank tensor to a uniaxial coordinate system.) Regrouping the summation in equation (4.24) and invoking the traceless property of the $\mathbf{S}$ matrix, we obtain

$$
\begin{aligned}
\bar{\delta} & =\delta \sum_{i} \frac{1}{3}\left(3 \cos ^{2} \gamma_{i}-1\right) S_{i i}^{P A S} \\
& =\delta\left[\begin{array}{l}
\frac{1}{2}\left(3 \cos ^{2} \gamma_{z}-1\right) S_{z z}^{P A S}-\frac{1}{6}\left(3 \cos ^{2} \gamma_{z}-1\right)\left(-S_{x x}^{P A S}-S_{y y}^{P A S}\right) \\
+\frac{1}{3}\left(3 \cos ^{2} \gamma_{x}-1\right) S_{x x}^{P A S}+\frac{1}{3}\left(3 \cos ^{2} \gamma_{y}-1\right) S_{y y}^{P A S}
\end{array}\right] \\
& =\delta\left[\begin{array}{l}
\frac{1}{2}\left(3 \cos ^{2} \gamma_{z}-1\right) S_{z z}^{P A S}+\left(\frac{1}{2} \cos ^{2} \gamma_{z}+\cos ^{2} \gamma_{x}-\frac{1}{2}\right) S_{x x}^{P A S} \\
+\left(\frac{1}{2} \cos ^{2} \gamma_{z}+\cos ^{2} \gamma_{y}-\frac{1}{2}\right) S_{y y}^{P A S}
\end{array}\right] . \\
& =\delta \frac{1}{2}\left[S_{z z}^{P A S}\left(3 \cos ^{2} \gamma_{z}-1\right)+\left(S_{x x}^{P A S}-S_{y y}^{P A S}\right)\left(\cos ^{2} \gamma_{x}-\cos ^{2} \gamma_{y}\right)\right]
\end{aligned}
$$

This result is equivalent to equation (4.21) after the directional cosines are converted to polar angles $\theta_{s}$ and $\phi_{s}$.

The expression for the motionally-averaged axially-symmetric interaction $\mathbf{T}$ in the $\mathbf{S}$ tensor PAS resembles the expression for the chemical shift of a randomly oriented sample in the laboratory frame (equation 2.60). The similarity is obvious if we define, for the $S$ matrix, an anisotropy parameter $\delta_{S}=S_{z z}^{P A S}$ and an asymmetry parameter $\eta_{s}=\left(S_{y y}^{P A S}-S_{x x}^{P A S}\right) / s_{z z}^{P A S}$, so that 


$$
\begin{aligned}
\bar{\delta} & =\frac{1}{2} \delta \cdot \delta_{s}\left[3 \cos ^{2} \gamma_{z}-1-\eta_{s}\left(\cos ^{2} \gamma_{x}-\cos ^{2} \gamma_{y}\right)\right] \\
& =\frac{1}{2} \delta \cdot \delta_{s}\left(3 \cos ^{2} \theta_{s}-1-\eta_{s} \sin ^{2} \theta_{s} \cos 2 \phi_{s}\right)
\end{aligned}
$$

The second form (4.22) of the relation between the averaged NMR anisotropy $\bar{\delta}$ and the $\mathbf{S}$ tensor is derived in the interaction tensor PAS,

$$
\begin{aligned}
\bar{\delta} & =\frac{2}{3} \sum_{i} S_{i i}^{T, P A S} T_{i i}^{P A S}=\frac{2}{3}\left[\delta \cdot S_{z z}^{T, P A S}-\frac{\delta}{2}\left(S_{x x}^{T, P A S}+S_{y y}^{T, P A S}\right)\right] \\
& =\delta \cdot S_{z z}^{T, P A S} \equiv \delta \cdot\left\langle\frac{1}{2}\left(3 \cos ^{2} \theta_{p d}-1\right)\right\rangle
\end{aligned}
$$

where $\theta_{p d}$ is the angle between the $T_{z z}^{P A S}$ axis and the director. This relation indicates that the order parameter $S_{z z}^{T, P A S}$ along the unique axis of $\mathbf{T}^{\mathrm{PAS}}$ is directly measurable as the ratio between the motionally-averaged NMR coupling $\bar{\delta}$ and the rigid-limit anisotropy $\delta$.

\subsection{Strategies for Determining Order Tensors}

A traceless and symmetric order matrix $\mathbf{S}$ contains five independent elements. By measuring at least five independent anisotropies of segment-fixed NMR interactions, a system of linear equations can be established to allow a complete solution of the $\mathbf{S}$ tensor. However, the number of unknowns can be reduced by assuming the presence of molecular motions and by taking into account the segmental symmetry.

If the molecules are rigid and rotate about a molecular axis, then two principal values of $\mathbf{S}$ for each segment are degenerate, i.e. $S_{y y}-S_{x x}=0$. The third principal value $S_{z z}=S_{m o l}$ is the same for all segments. In other words, there is a single uniaxial $\mathbf{S}$ tensor for the whole molecule. Using equation (4.21), we can relate the order parameter of a bond $S_{b o n d}$ to the order parameter of the molecular axis $S_{m o l}$ through the angle $\theta_{p m}$ between the bond and the molecular axis, 


$$
S_{b o n d, \theta_{p d}} \equiv \bar{\delta} / \delta=P_{2}\left(\cos \theta_{p m}\right) \cdot S_{m o l, \theta_{m d}}
$$

As indicated by the subscripts, $\theta_{p d}$ refers to the angle between the bond and the director, whereas $\theta_{m d}$ specifies the angle between the molecular axis and the director. If $S_{m o l}$ is known and the averaged coupling $\bar{\delta}$ is obtained experimentally, then one can derive the angle $\theta_{p m}$ between the bond and the molecular rotation axis.

In the past, relation (4.28) between $S_{b o n d}$ and $S_{m o l}$ has been used conversely in interpreting the ${ }^{2} \mathrm{H} \mathrm{NMR}$ results. It was claimed that $S_{C H}=-\frac{1}{2} S_{m o l}$, so that the experimental ${ }^{2} \mathrm{H}$ quadrupolar splittings could be converted to a $S_{m o l}$ profile for the acyl chain. These calculations require the inherent assumptions that the orientation of the molecular rotation axis is known and that the $\mathrm{C}-2 \mathrm{H}$ bonds are perpendicular to this axis everywhere. The validity of these assumptions have not been proven. Moreover, even with these simplifying assumptions, it has been shown that the headgroup structure of phospholipids cannot be determined from the ${ }^{2} \mathrm{H}$ and ${ }^{31} \mathrm{P}$ NMR data alone. ${ }^{6}$

Among the five independent non-zero elements of a $S$ tensor, three elements determine the orientation of the PAS and the other two define the principal values. For small rigid molecules, the orientation of the $\mathbf{S}$ PAS can be partially or completely determined based on molecular symmetries. ${ }^{7}$ If the molecule possesses $C_{n}(n>2)$ symmetry, then the $z$-axis of the S PAS is along the $C_{n}$ axis, whereas the $x$ - and $y$-axes are equivalent in the transverse plane. Thus only one independent element needs to be determined. If two mutually perpendicular mirror planes are present in the molecule, then the $z$-axis of the S PAS is the intersection of the two planes while the other two axes lie in the two planes. With all orientational parameters thus determined, two independent unknowns are left to describe the principal values. If the molecule possesses one symmetry plane, then the $z$-axis is perpendicular to that plane and three non-zero elements remain, one fixing the orientation and the other two the magnitudes. Finally, for a perfect tetrahedron, all elements of the $\mathbf{S}$ matrix are zero. However, for methylene groups in a 
complex molecule, only the tetrahedral angle but not the tetrahedral bond length is preserved, therefore the only useful simplifying feature is that the sum of the order parameters along the four bonds of a tetrahedron vanishes. Additionally, it is generally true that the sum of order parameters along three orthogonal directions also vanishes.

Previous ${ }^{2} \mathrm{H}$ NMR studies indicated that the-two ${ }^{2} \mathrm{H}$ quadrupolar couplings of the methylene groups in the phospholipid headgroup and glycerol segments are nearly degenerate, even when their magnitudes are drastically changed due to electric charges incorporated into the bilayers. ${ }^{8}$ The model to explain this degeneracy involves a fast conformational exchange of the molecule between one or several pairs of mirror-symmetric headgroup structures so that the geminal protons effectively interchange. ${ }^{9,10}$ According to this exchange model, the plane perpendicular to the $\mathrm{H}-\mathrm{H}$ vector is a symmetry plane of the segment, so one of the principal axes of the $\mathrm{S}$ tensor must be parallel to the $\mathrm{H}-\mathrm{H}$ vector. This reduces the number of unknowns in the order tensor to three. Two specify the principal values and the third the angle that defines the principal axis in the symmetry plane. This headgroup exchange model is discussed in more detail in section 6.5.

\subsection{Orientation Distributions from Order Tensors}

In section 4.3, we introduced order parameter in terms of the orientation distribution of rod-shaped molecules about a nematic axis. We also mentioned that the orientational order of any arbitrarily-shaped molecules in a uniaxial LC phase could be analyzed using the full order tensor $\mathbf{S}$. We now explore this second statement for the case of phospholipids. The macroscopic uniaxiality of bilayer phospholipids induced by their motions ensures that segmental orientations can be described equivalently but conversely by the orientation distribution $P(\theta, \phi)$ of the director with respect to a segment-fixed frame. To derive $P(\theta, \phi)$ from the $\mathbf{S}$ tensor, we again employ the second-order spherical harmonics $Y_{2, M}(\theta, \phi)$, where the polar angles $\theta$ and $\phi$ specify the director axis in the $\mathbf{S}$ tensor.PAS. The principal value $z$ of the order tensor is 


$$
\begin{aligned}
S_{z z} & =\frac{1}{2}\left\langle 3 \cos ^{2} \theta-1\right\rangle=\sqrt{\frac{4 \pi}{5}}\left\langle Y_{2,0}(\theta, \phi)\right\rangle \\
& =\sqrt{\frac{4 \pi}{5}} \int_{0}^{2 \pi} d \phi \int_{0}^{\pi} d \theta \sin \theta P(\theta, \phi) \times Y_{2,0}(\theta, \phi)
\end{aligned} .
$$

The transverse principal values of the $\mathbf{S}$ tensor follow the relation

$$
\begin{aligned}
S_{y y}-S_{x x} & =\frac{1}{2}\left\langle 3 \cos ^{2} \gamma_{y}-1\right\rangle-\frac{1}{2}\left\langle 3 \cos ^{2} \gamma_{x}-1\right\rangle \\
& =\frac{1}{2}\left\langle\sin ^{2} \theta \cos (2 \phi)\right\rangle \\
& =\sqrt{\frac{2 \pi}{15}}\left(\left\langle Y_{2,2}(\theta, \phi)\right\rangle+\left\langle Y_{2,-2}(\theta, \phi)\right\rangle\right)
\end{aligned} .
$$

The averages $\left\langle Y_{L, M}(\theta, \phi)\right\rangle$ appear as the coefficients in the expansion of the distribution function $P(\theta, \phi)$ in the basis of the spherical harmonics, ${ }^{11,12}$

$$
P(\theta, \phi)=\sum_{L=0}^{\infty} \sum_{M=-L}^{L}\left\langle Y_{L, M}(\theta, \phi)\right\rangle Y_{L, M}(\theta, \phi)
$$

The first term in the series $Y_{0,0}=1 / \sqrt{4 \pi}$ is isotropic. All terms for odd $L$ vanish due to the symmetry of the uniaxial system under inversion of the director, as shown for $L$ $=1$ (4.17). Thus only even order terms in the expansion remain. Truncating the series at $L=2$, we obtain a low-resolution approximation $P^{(2)}(\theta, \phi)$ of $P(\theta, \phi)$, which can be expressed by the elements of the $\mathbf{S}$ matrix in its PAS

$$
\begin{aligned}
& P^{(2)}(\theta, \phi)=\left\langle Y_{0,0}\right) Y_{0,0}+\left\langle Y_{2,0}(\theta)\right\rangle Y_{2,0}(\theta)+ \\
& \left\langle Y_{2,2}(\theta, \phi)\right\rangle Y_{2,2}(\theta, \phi)+\left\langle Y_{2,-2}(\theta, \phi)\right\rangle Y_{2,-2}(\theta, \phi) \\
= & \sqrt{\frac{1}{4 \pi}} Y_{0,0}+\sqrt{\frac{5}{4 \pi}} S_{z z} Y_{2,0}+\frac{1}{2} \sqrt{\frac{15}{2 \pi}}\left(S_{y y}-S_{x x}\right) \cdot\left(Y_{2,2}(\theta, \phi)+Y_{2,-2}(\theta, \phi)\right)
\end{aligned}
$$

Here a term $\frac{1}{2} S_{x y}\left(Y_{2,2}(\theta, \phi)-Y_{2,-2}(\theta, \phi)\right)$ vanishes because $S_{x y^{\prime}}=0$ in the $S$ tensor PAS. 
Figure 4.5a shows an example of a distribution $P(\theta, \phi)$ with four maxima, represented by contour lines on a unit sphere. The corresponding $P^{(2)}(\theta, \phi)$ (Figure 4.5b) is only a low-resolution approximation to the original distribution. In addition, for order tensors with large principal values, the $P^{(2)}(\theta, \phi)$ approximation contains negative values, which are unphysical. Better approximations which are positive everywhere can be made using model distributions described below.

Since three out of five independent parameters needed for a complete determination of the order tensor parameterize the orientation of the distribution, the two remaining parameters adjust for the shape of the distribution. These two parameters are directly related to $S_{z z}$ and $\left|S_{y y}-S_{x x}\right|$. In the following, we describe two model distributions based on different combinations of these shape parameters.

\section{Double Gaussian Distribution}

A physically reasonable distribution with two shape parameters is the sum of two axially symmetric Gaussians of identical width $\sigma$, with their centers separated by an angle $\beta$, as shown in Figure 4.5c. The splitting $\beta$ and the width $\sigma$ of the Gaussians are the two shape parameters of the distribution. Defining the orientational second moment of the individual Gaussian as $S_{m o l}$, the principal order parameters of the total distribution fulfill the relations

$$
\begin{gathered}
S_{z z}=S_{m o l} \cdot \frac{1}{2}\left(3 \cos ^{2} \beta-1\right) \\
\left|S_{y y}-S_{x x}\right|=S_{m o l} \cdot \frac{3}{2} \sin ^{2} \beta .
\end{gathered}
$$

The relation between the Gaussian width $\sigma$ and the order parameter $S_{m o l}$ has been calculated and displayed elsewhere. ${ }^{12}$. It is also contained in Figure 4.6, where $S_{m o l}$ can be read from the $S_{z z}$ axis for $\sigma_{x}=\sigma_{y}=\sigma$. For a uniaxial order tensor, $\left|S_{y y}-S_{x x}\right|=0$ and 


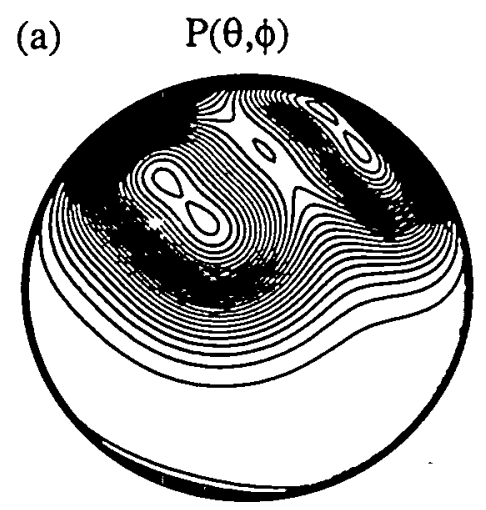

(c) double Gaussian

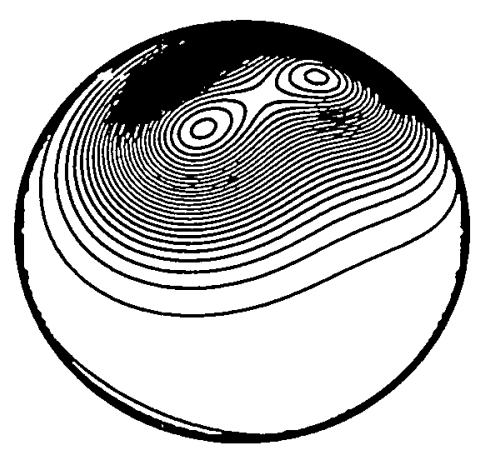

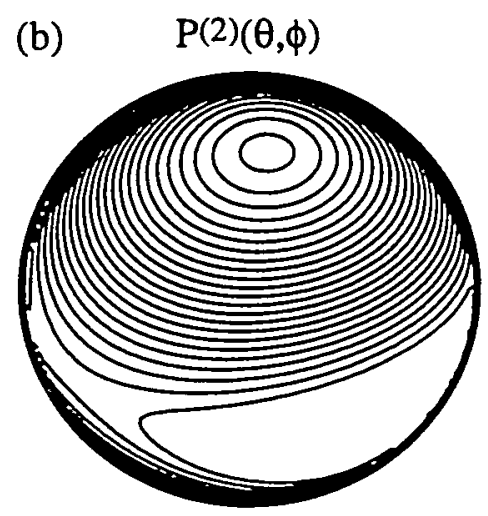

(d) biaxial Gaussian

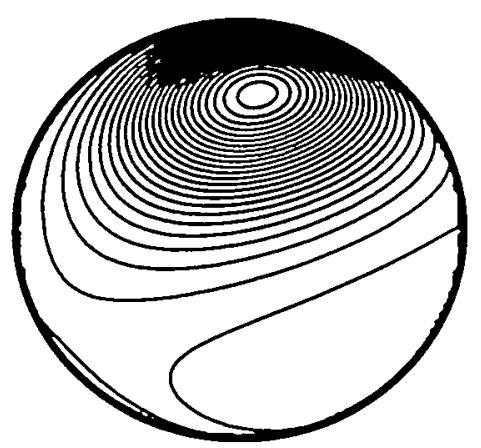

Figure 4.5 Director distributions in a segment-fixed frame. The distributions correspond to order parameters $S_{z z}=0.4776$ and $S_{y y}-S_{x x}=0.1206$. (a) Original distribution $P(\theta, \phi)$. (b) $P^{(2)}(\theta, \phi)$ approximation, obtained from the principal values of the order tensor. (c) and (d) Five-parameter distributions which reproduce the same order tensor as the original distribution. (c) Double Gaussian approximation. (d) Single biaxial Gaussian approximation. 
$\beta=0$. Therefore the two Gaussians merge into one to create a uniaxial distribution as required.

The double Gaussian distribution is closely related to the model of exchange between two mirror-symmetric conformations, where $S_{m o l}$ corresponds to the molecular order parameter. This is discussed later for the case of headgroup exchange in phospholipids.

\section{Single Biaxial Gaussian Distribution}

A uniaxial Gaussian on a sphere with its center at the north pole is described by

$$
P(\theta, \phi)=\exp \left(-\frac{\sin ^{2} \theta}{2 \sigma^{2}}\right)=\exp \left(\frac{-x^{2}-y^{2}}{2 \sigma^{2}}\right)
$$

where $x=\sin \theta \cos \phi$ and $y=\sin \theta \sin \phi$ are the components of a unit vector. This equation can be generalized to produce a biaxial Gaussian,

$$
P(\theta, \phi)=\exp \left(-\frac{x^{2}}{2 \sigma_{x}^{2}}-\frac{y^{2}}{2 \sigma_{y}^{2}}\right)
$$

The broadenings $\sigma_{x}$ and $\sigma_{y}$ are the two shape parameters of this distribution. Figure $4.5 \mathrm{~d}$ plots the biaxial Gaussian approximation to the orientation distribution $P(\theta, \phi)$. Compared to the $P^{(2)}(\theta, \phi)$ approximation, it represents the features of the original distribution with higher resolution. Figure 4.6 shows the relation of the shape parameters $\left(\sigma_{x}, \sigma_{y}\right)$ of the biaxial Gaussian with the principal order parameters $S_{z z}$ and $S_{y y}-S_{x x}$, obtaine by numerical integration. The limiting values of $S_{y y}-S_{x x}$ are found to be \pm 0.75 and occur when $\left(\sigma_{x} \rightarrow \infty, \sigma_{y} \rightarrow 0\right)$ and $\left(\sigma_{x} \rightarrow 0, \sigma_{y} \rightarrow \infty\right)$. This can be understood as follows: on the unit sphere, the biaxial Gaussian with widths of $\sigma_{x} \rightarrow \infty$ and $\sigma_{y} \rightarrow 0$ has the shape of a narrow ring in the $x z$ plane, perpendicular to the $S_{y y}$ axis. With this extreme 
distribution, all directors are oriented at $90^{\circ}$ from the $y$-axis, so the principal value $S_{y y}$ is $S_{y y}=P_{2}\left(\cos 90^{\circ}\right)=-0.5$. Since the distribution is uniaxial around the $S_{y y}$ axis, $S_{z z}=S_{x x}$ $=-0.5$, and $S_{y y}=+0.25$. Thus $S_{y y}-S_{x x}=-0.75$. The case for $\sigma_{y} \rightarrow \infty$ and $\sigma_{x} \rightarrow 0$ is completely analogous and yields $S_{y y}-S_{x x}=+0.75$.
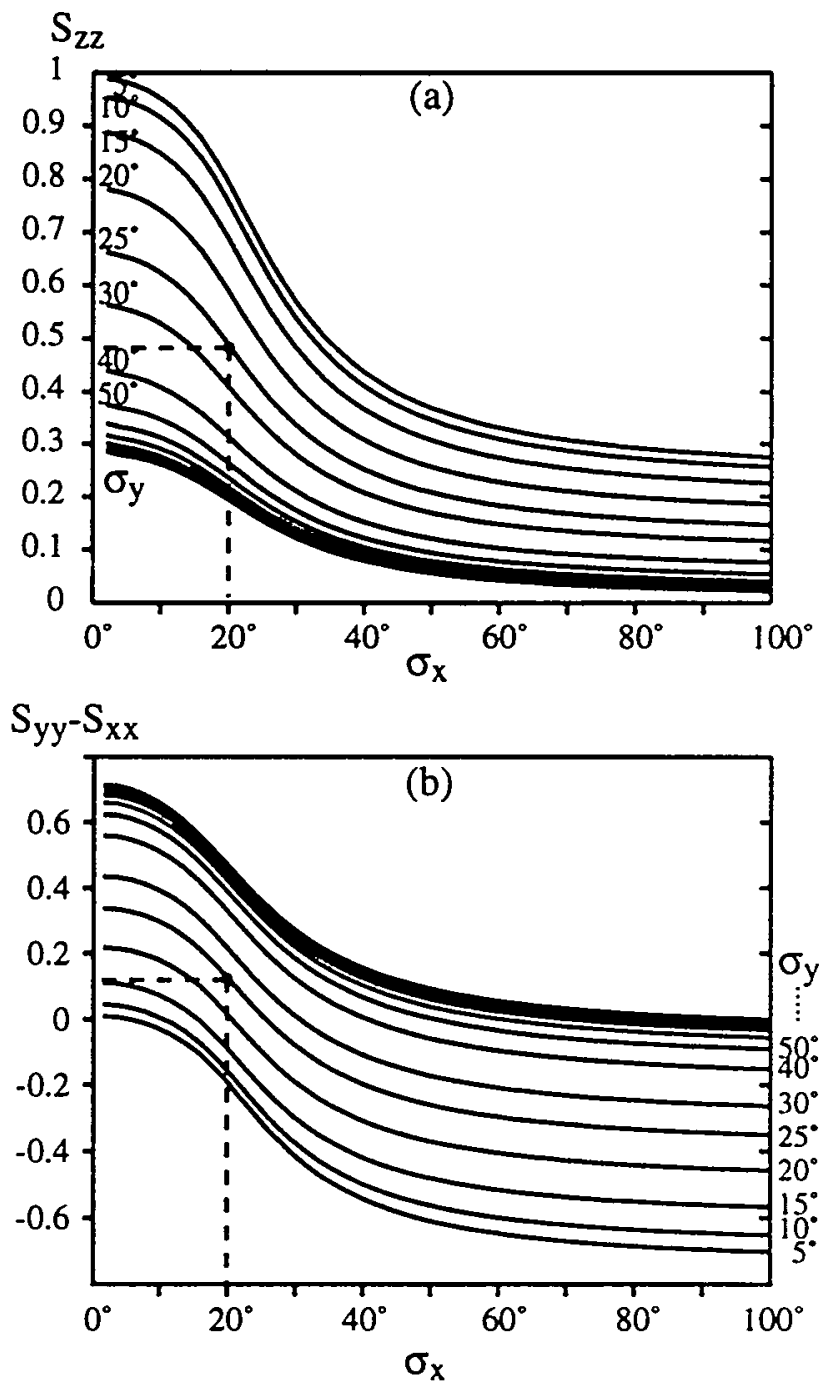

Figure 4.6 Relation of principal order parameters with shape parameters of a biaxial Gaussian distribution. (a) $S_{z z}$ and (b) $S_{y y}-S_{x x}$. The shape parameters $\sigma_{x}$ and $\sigma_{y}$ can be determined from the order parameters by finding a pair of $\sigma_{x}$ and $\sigma_{y}$ in both plots that is consistent with the order parameters. Dashed lines: example for the order parameters in Figure 4.5. 
The width parameters of the two model distributions described here are related by $\min \left(\sigma_{x}, \sigma_{y}\right) \equiv \sigma$, and $\max \left(\sigma_{x}, \sigma_{y}\right) \approx \sqrt{(\beta / 2)^{2}+\sigma^{2}}$. This is borne out by the parameters of the distributions shown in Figure 4.5.

\subsection{Probability Limits from Bond Order Parameters}

So far, we demonstrated how model distributions can be parameterized by the segmental order tensor. Next we describe an approach for utilizing bond order parameters, or the projections of the order tensor along the bonds, to obtain model-independent probabilities of bond orientations.

\section{Model-Independent Probabilities from Negative Order Parameters}

Consider the order parameter of the $\mathrm{C}-\mathrm{H}$ bond, obtained from the motionallyaveraged $\mathrm{C}-\mathrm{H}$ dipolar couplings or the $2 \mathrm{H}$ quadrupolar splittings ${ }^{13-16}$ according to equation (4.22). These order parameters contain information about the probability distribution of the angle between the $\mathrm{C}-\mathrm{H}$ bond and the bilayer director. Particularly, $S_{\mathrm{CH}}$ close to the limiting values of 1 and $-1 / 2$ contains much more information than the $L \leq 2$ approximation in the $Y_{L, M}$ expansion suggests. For instance, when $S_{C H}=-0.5$, the $\theta$ dependence of the orientation distribution is fully known: it is a single narrow peak at $\theta=$ $90^{\circ}$. By inference, when $S_{C H}$ is close to -0.5 , the orientation distribution will be high near $\theta=90^{\circ}$ and low near $\theta=0^{\circ}$. Based on this insight, we ask the following question: given a negative order parameter, what is the upper limit to the probability of finding the bond oriented at small angles with respect to the director?

In the following, we consider only the $\theta$ dependence of the orientation distribution. It is obtained from the two-parameter distribution $P(\theta, \phi)$ by integrating over the angle $\phi$,

$$
p(\theta)=\frac{1}{2 \pi} \int_{0^{\circ}}^{2 \pi} P(\theta, \phi) \sin \theta d \phi
$$


The bond order parameter $S_{C H}$ constrains the integral of $p(\theta)$ according to

$$
S_{C H}=\int_{0^{\circ}}^{90^{\circ}} p\left(\theta^{\prime}\right) P_{2}\left(\cos \theta^{\prime}\right) d \theta^{\prime}
$$

Equation (4.37) suggests that the order parameter may yield information on the integral probability $I(\theta)$ of finding the bonds oriented at small angles from the director,

$$
I(\theta)=\int_{0^{\circ}}^{\theta} p\left(\theta^{\prime}\right) d \theta .
$$

Several examples of probability distributions $p(\theta)$ and their integral probabilities $I(\theta)$, which all give rise to the same bond order parameter $S_{C H}=-0.2$, are shown in Figure 4.7. As seen, it is impossible to find a model-independent limit for $p(\theta)$ on the basis of equation (4.37): $p\left(\theta^{\prime}\right)$ can in principle exhibit high and narrow spikes of small area that contribute little to the integral for $S_{C H}$. In comparison, $I(\theta)$ is a steady function confined to values between 0 and 1 . Nevertheless, there are still multiple $I(\theta)$ functions for a given $S_{C H}$.

Our objective, then, is to find an upper-limit function, $I_{\lim }(\theta) \geq I(\theta)$, that provides as stringent a limit as possible to any $I(\theta)$ for a given negative order parameter. This is equivalent to searching for an extreme distribution $p_{\text {lim }}(\theta)$ that

(a) produces the maximum possible $I(\theta)$, according to equation (4.38),

(b) yields the observed negative $S_{C H}$ value, according to equation (4.37), and

(c) is normalized to unity.

Since $S_{C H}$ is negative, the distribution $p(\theta)$ must contain a major peak at angles with negative $P_{2}(\cos \theta)$. On the other hand, the bond orientation may very well fluctuate, adopting smaller angles $\theta^{\prime}$ with positive $P_{2}(\cos \theta)$. However, to produce the negative $S_{C H}$, the probability for the smaller angles must have an upper limit. The probability $I(\theta)$ 


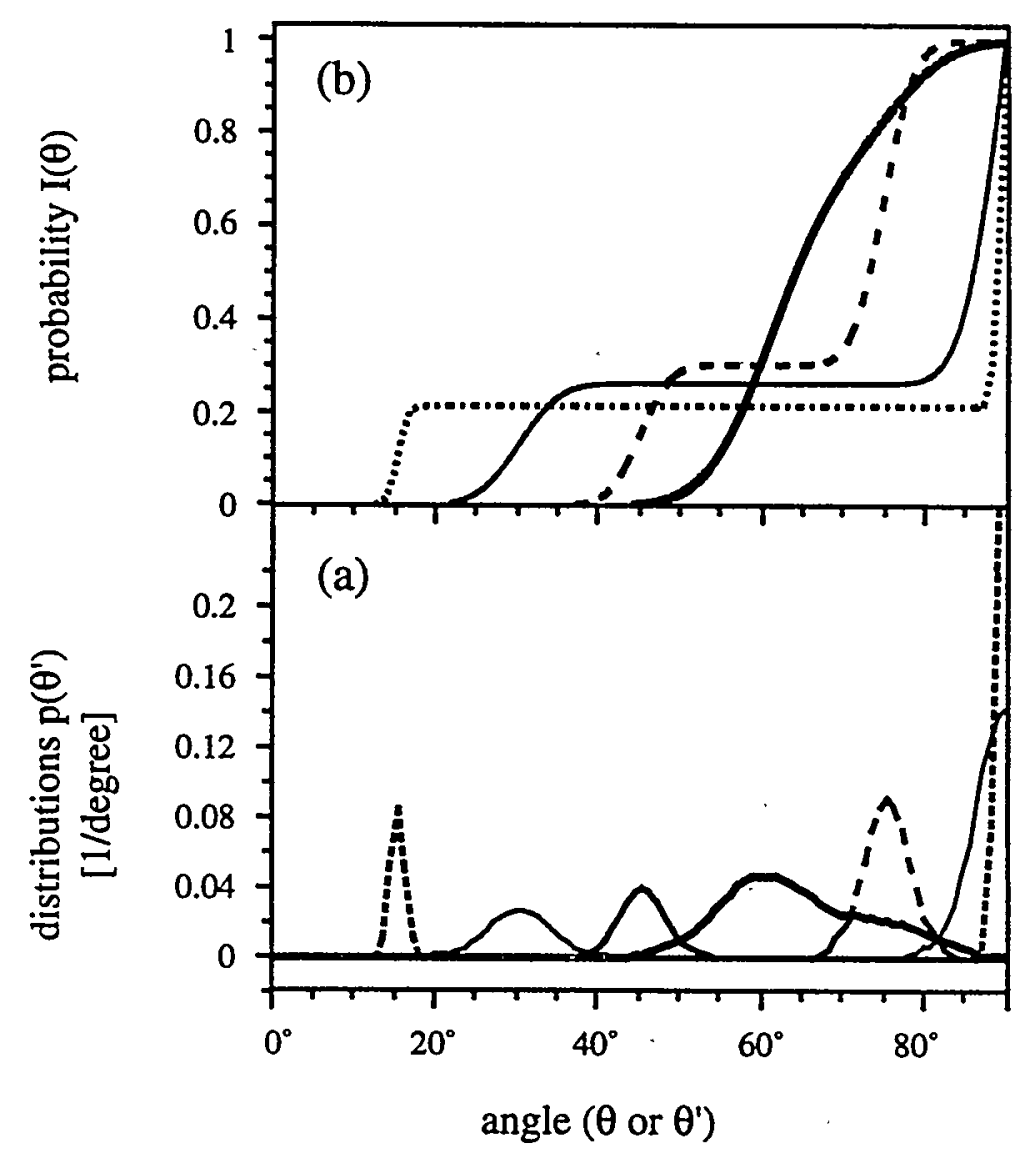

Figure 4.7 Orientation distributions and probabilities constrained by bond order parameter. (a) Distributions $p\left(\theta^{\prime}\right)$ with $S_{C H}=-0.2$. (b) Corresponding integral probabilities $I(\theta)$.

at small angles or large positive $P_{2}(\cos \theta)$ values is the largest if its contribution to $S_{C H}$ is compensated for by a large $p(\theta)$ density in the regions where $P_{2}(\cos \theta)$ is very negative. In the limiting case that we are looking for, $p_{\lim }(\theta)$ for $\theta^{\prime}>\theta$ is a single sharp peak at $\theta^{\prime}=$ $90^{\circ}$. On the other hand, in the region $\theta \leq \theta$, to maximize $I(\theta)$ while keeping $\theta$ $\int_{0^{\circ}}^{\theta} p\left(\theta^{\prime}\right) P_{2}\left(\cos \theta^{\prime}\right) d \theta^{\prime}$ constant, $p_{\lim }(\theta)$ must be concentrated at the angle of the smallest allowed $P_{2}(\cos \theta)$. In other words, $p_{\lim }(\theta)$ must contain a narrow peak at $\theta$. The resulting extreme distribution is 


$$
p_{\lim }(\theta)=I_{\lim }(\theta) \delta(\theta-\theta)+\left(1-I_{\lim }(\theta)\right) \delta\left(\theta-90^{\circ}\right),
$$

and is shown in Figure 4.8a for $\theta=45^{\circ}$. Thus, equation (4.37) becomes

$$
S_{C H}=I_{\lim }(\theta) P_{2}(\cos \theta)+\left(1-I_{\lim }(\theta)\right) P_{2}\left(\cos 90^{\circ}\right) .
$$

This yields the limit for the integral probability:

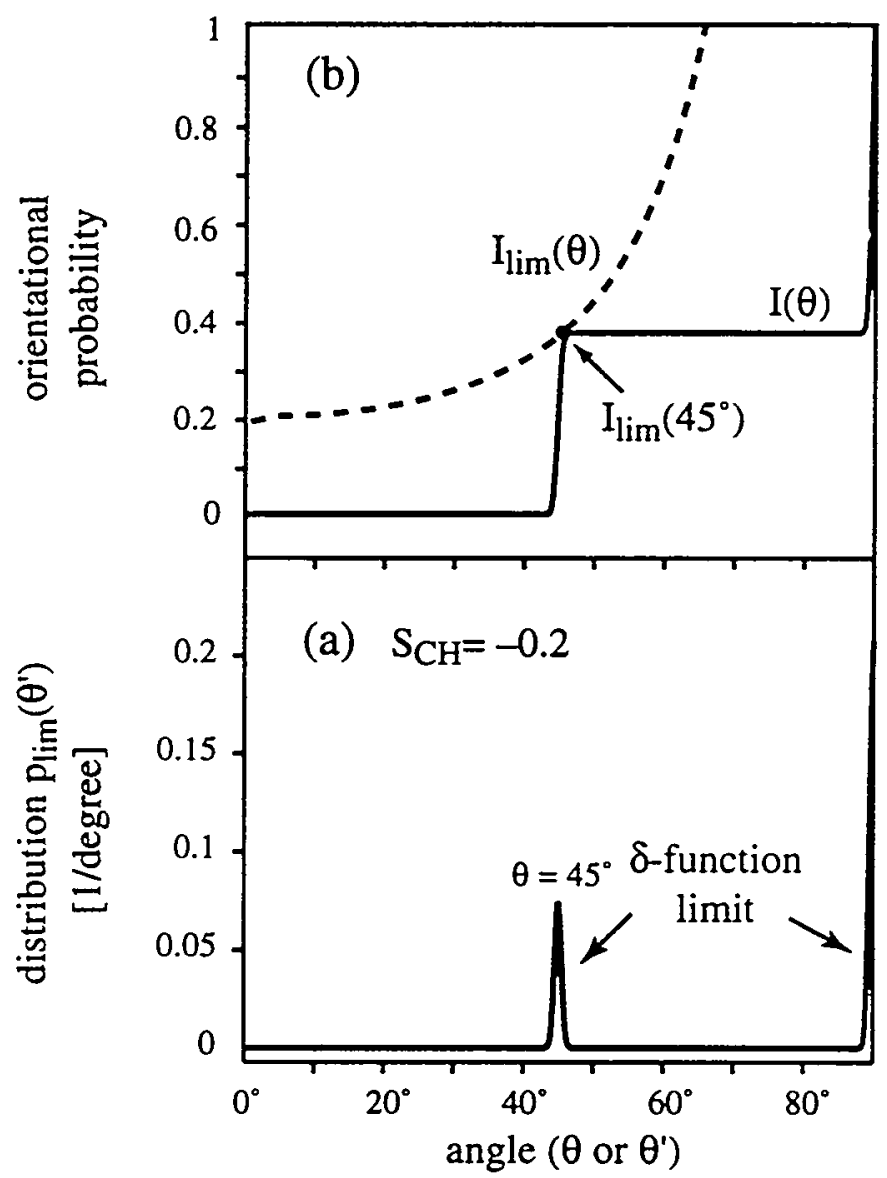

Figure 4.8 Model-independent upper-limit probabilities for a negative order parameter. (a) Example limiting distribution $p_{\text {lim }}\left(\theta^{\prime}\right)$ with $S_{C H}=-0.2$. (b) Corresponding integral probabilities $I(\theta)$ (solid line) and $I_{\lim }(\theta)$ (dashed line). $I(\theta)$ provides a point on the $I_{\text {lim }}(\theta)$ curve, at the $\theta$ value of the left peak in $p_{\lim }(\theta)$. 


$$
I(\theta) \leq I_{\lim }(\theta)=\frac{S_{C H}+0.5}{P_{2}(\cos \theta)+0.5}
$$

The model-independent upper-limit probability function, corresponding to an order parameter of $S_{C H}=-0.2$, is shown as the dashed line in Figure 4.8b. The solid line in the figure is the integral $I(\theta)$ of the representative distribution $\eta_{\lim }(\theta)$ in Figure 4.8a. These plots indicate how the step of $I(\theta)$ determines the value of the $I_{\lim }(\theta)$ curve at $45^{\circ}$.

The model-independent upper-limit functions for a series of $S_{C H}$ values between 0.5 and -0.5 are calculated and plotted in Figure 4.9.

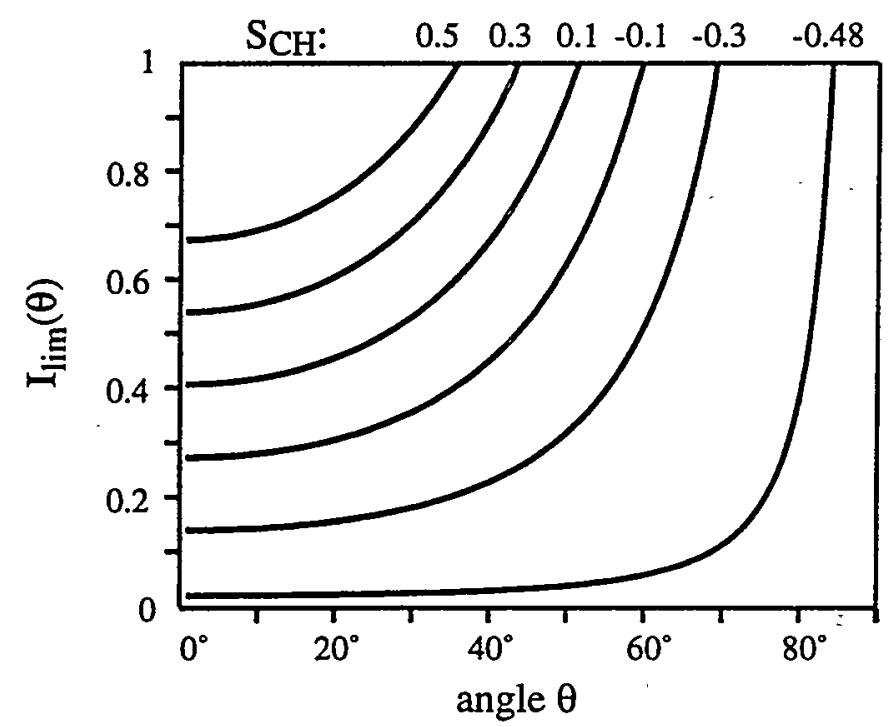

Figure 4.9 Upper-limit probabilities parameterized by bond order parameter. The maximum probability $I_{\lim }(\theta)$ of finding the bond at small angles with respect to the director can be read off directly from one of the order parameter $\left(S_{\mathrm{CH}}\right)$ curves in the plot.

Probability Limits under the Assumption of Broadening

So far, the upper-limit function is found for a very unphysical distribution consisting of two extremely narrow peaks. By assuming a reasonable broadening of the distribution maxima, we can obtain an even more stringent upper limit to the integral 
probability. A standard deviation $\sigma$ of approximately $10^{\circ}$, corresponding to a molecular order parameter of $0.9,{ }^{12}$ appears as a safe minimum for the broadening in liquid crystals. In our calculations, we use Gaussian peaks:

$$
\begin{aligned}
& P(\theta, \phi)=2 \pi \cdot c \cdot \sum_{j} e^{-\left(\theta-\theta_{j}\right)^{2} / 2 \sigma_{j}^{2}} \\
& \Rightarrow p(\theta)=c \cdot \sin \theta \sum_{j} e^{-\left(\theta-\theta_{j}\right)^{2} / 2 \sigma_{j}{ }^{2}}
\end{aligned}
$$

with $p(\theta)$ normalized to unity by the constant $c$. The results do not depend strongly on the exact functional form of $p(\theta)$, as long as the standard deviations of the individual peaks are equal to $\sigma$.

It is difficult to find an analytical expression for the most stringent upper-limit probability function $I_{\lim }^{\sigma}(\theta)$ under the assumption of broadening. So instead, we perform a numerical calculation of $I_{\lim }^{\sigma}(\theta)$. Again, the limiting distribution $p_{\lim }^{10^{\circ}}\left(\theta^{*}\right)$ consists of only two peaks (Figure 4.10a), one of which is centered at $\theta^{\prime}=90^{\circ}$. The other peak has its maximum at an angle somewhat smaller than $\theta$. A peak centered at $\theta$ will not produce the largest possible $I(\theta)=\int_{0^{\circ}}^{\theta} p\left(\theta^{\prime}\right) d \theta^{\prime}$, since only about $50 \%$ of that peak contributes to the integral. Thus, the integral is larger if the peak is centered at an angle $\theta_{c}<\theta$, even though the amplitude of the peak is smaller by approximately $P_{2}\left(\cos \theta_{c}\right) / P_{2}(\cos \theta)$. Although the limiting distribution contains only two peaks, the resulting $I_{\lim }^{\sigma}(\theta)$ applies for any $p\left(\theta^{\prime}\right)$ consisting of any number of Gaussian peaks with widths larger than $\sigma$. Shown in Figure $4.10 \mathrm{~b}$ as a dashed line is the $I_{\lim }^{\sigma}(\theta)$ curve with $\sigma=10^{\circ}$. It exhibits lower values than the $I_{\lim }(\theta)$ curve at all angles, providing a more stringent upper limit to the probability of finding the bond below a certain angle with respect to the director. The quadratic $\theta$ dependence of $I_{\lim }^{\sigma}(\theta)$ at $\theta<<\sigma$ is due to the factor $\sin \theta$ in the $p(\theta)$ function, and to the fact that for $\theta \ll \sigma$ the integration covers only a part of the peak. 


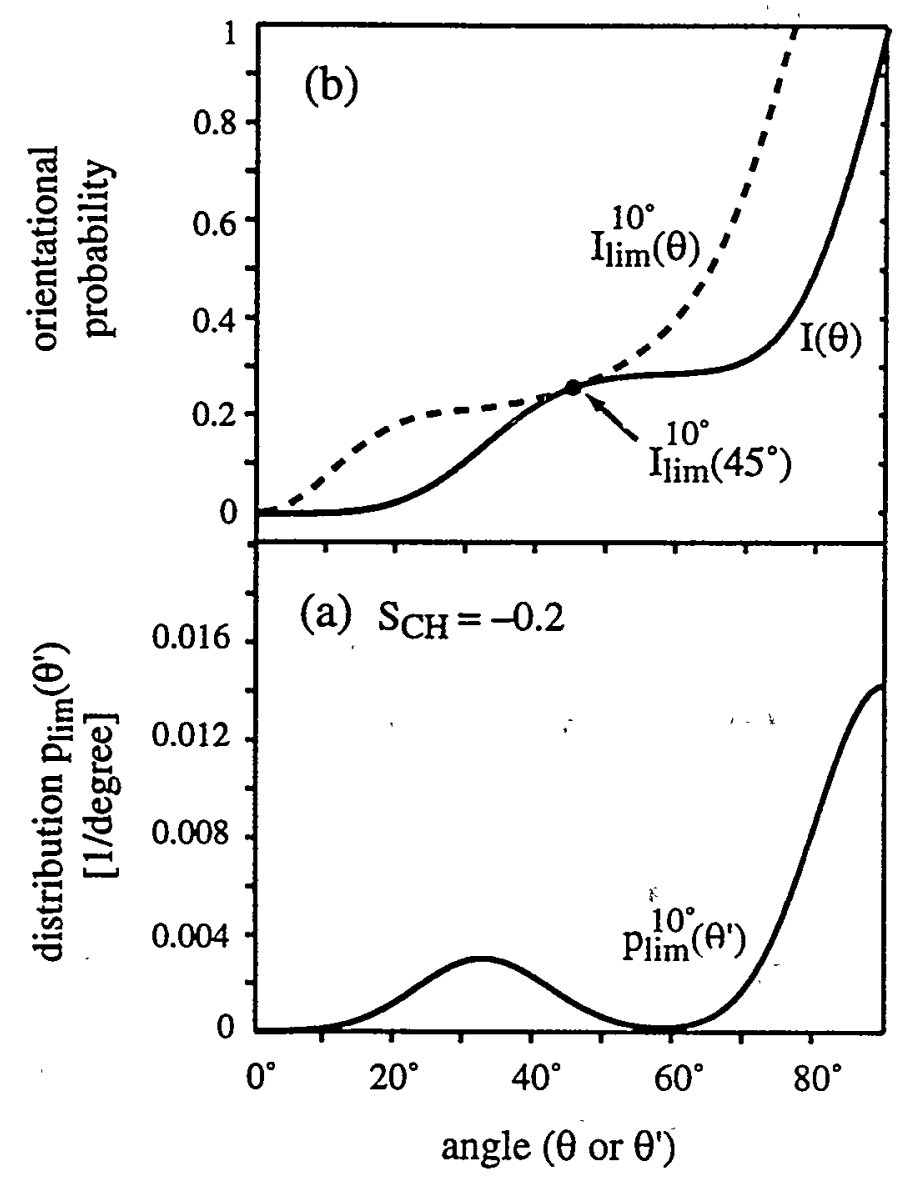

Figure 4.10 Upper-limit probabilities for broadened distributions. (a) Example limiting distribution $p_{\lim }^{10^{\circ}}\left(\theta^{\prime}\right)$ with $10^{\circ}$ minimal broadening and $S_{C H}=-0.2$. (b) The corresponding integral probability $I(\theta)$ (solid line) and upper-limit probability $I_{\lim }^{10^{\circ}}(\theta)$ (dashed line).

A set of $I_{\lim }^{\sigma}(\theta)$ curves for different broadenings of the distribution, all corresponding to a bond order parameter of -0.22 , is shown in Figure 4.11 .

\section{Probability Limits from Positive Order Parameters}

For positive order parameters, similar considerations can be made to determine the integral probability between $90^{\circ}$ and a minimum angle $\theta$, 


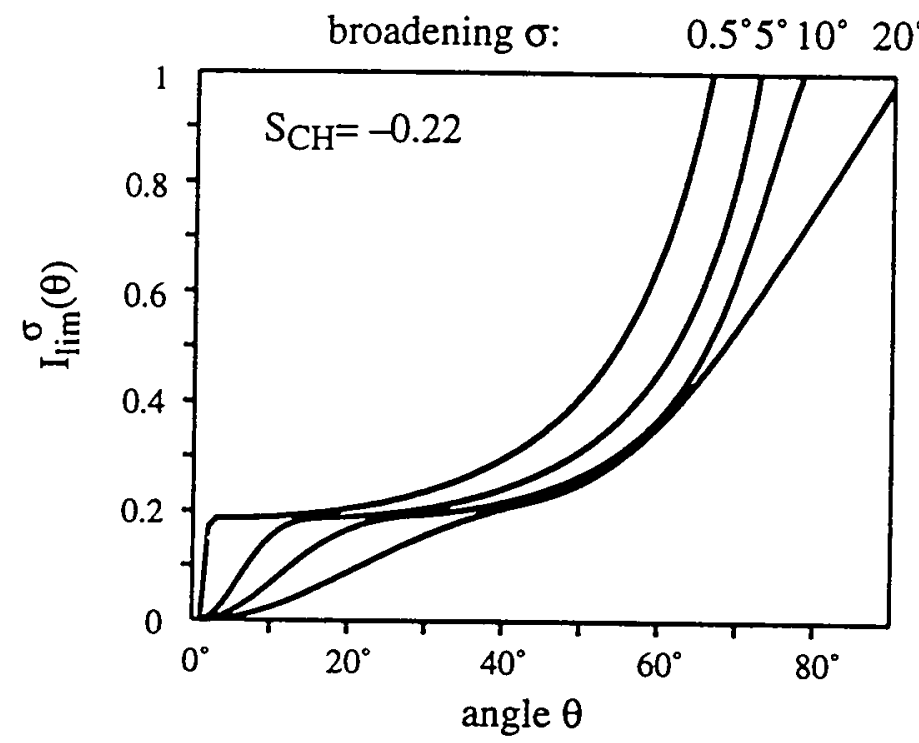

Figure 4.11 Upper-limit probabilities parameterized by minimum Gaussian broadening $\sigma$. The order parameter $S_{C H}=-0.22$.

$$
I_{90}(\theta)=\int_{\theta}^{90^{\circ}} p(\theta) d \theta
$$

In this case, $p\left(0^{\circ}\right) P_{2}\left(\cos 0^{\circ}\right)$ compensates for the contributions of negative $P_{2}(\cos \theta)$ to $S_{C H}$. Analogous to equation (4.40), the upper limit to the probability of finding the C-H bonds above an angle $\theta$ with respect to the director is determined by

$$
S_{P C}=I_{90, \lim }(\theta) P_{2}(\cos \theta)+\left(1-I_{90, \lim }(\theta)\right) P_{2}\left(\cos 0^{\circ}\right)
$$

The subscript " $\mathrm{PC}$ " is used because large positive order parameters are observed for $\mathrm{P}-\mathrm{C}$ dipolar couplings in phosphocholine. ${ }^{17}$ Equation (4.44) yields

$$
I_{90}(\theta) \leq I_{90, \lim }(\theta)=\frac{1-S_{P C}}{1-P_{2}(\cos \theta)}
$$


The integral-limit probability curve for $S_{P C}=+0.4$, which is found for the vector connecting the ${ }^{31} \mathrm{P}$ atom and the G3 carbon in phosphocholine, is shown in Figure 4.12a (solid line). Although not as stringent as that for $S_{C H}=-0.2$, it still shows that the angle between the $\mathrm{P}-\mathrm{G} 3$ vector and the director is preferentially smaller than $63^{\circ}$.

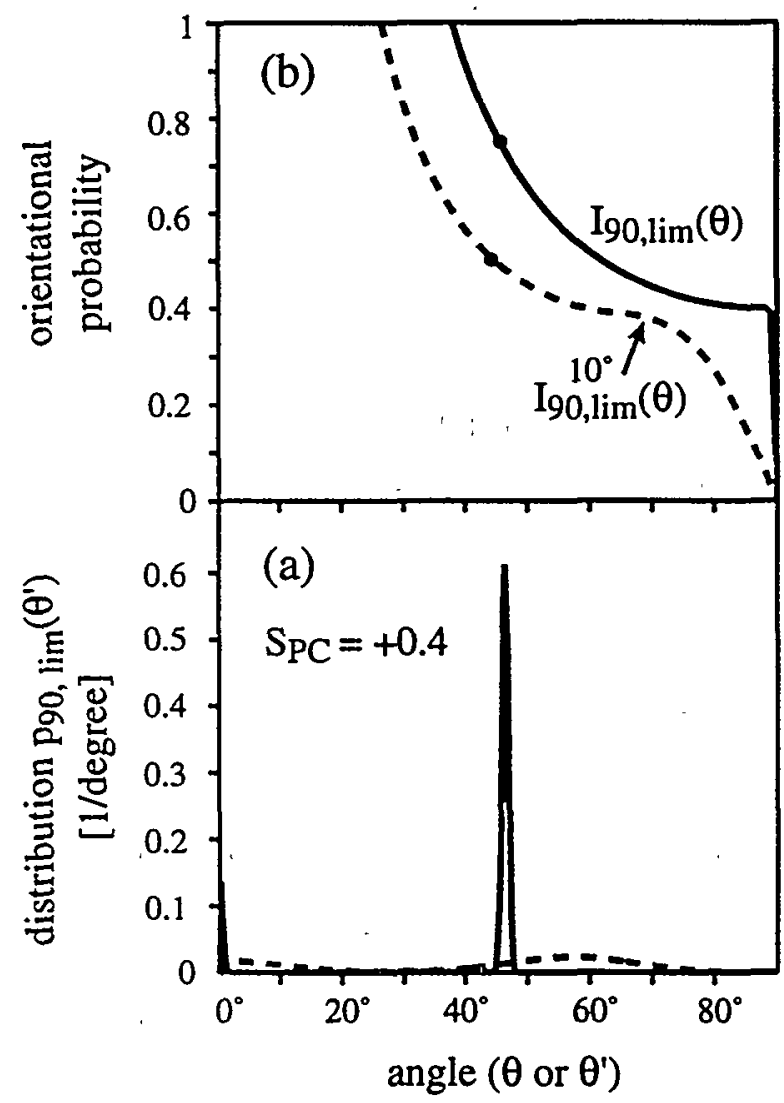

Figure 4.12 Upper-limit probabilities for a positive order parameter. (a) Example limiting distributions $p 90, \mathrm{lim}\left(\theta^{\prime}\right)$ and (b) corresponding upper-limit probabilities $I_{90, \lim }(\theta)$ for $S_{P C}=+0.4$. The distribution integrals yield the points at $\theta=45^{\circ}$ on the $I_{90, \lim }(\theta)$ curves. Solid lines: model-independent case. Dashed lines: with $10^{\circ}$ minimum Gaussian broadening.

When a minimal broadening is assumed for the distribution, the integral probability curve $I_{90, \mathrm{lim}}^{\sigma}(\theta)$ is shifted to smaller angles, as shown in Figure 4.12a by the dashed line. Thus, the upper limit of the integral probability of finding the bond orientation between an 
angle $\theta$ and $90^{\circ}$ is more stringent. Examples of the probability density functions $p_{90, \text { lim }}^{\sigma}(\theta)$ determining the limiting values at $\theta=45^{\circ}$ are shown in Figure $4.12 \mathrm{~b}$. According to their integrals, maximum probabilities of the bond being oriented at angles larger than $45^{\circ}$ are $76 \%$ in the case of the delta function limit and $50 \%$ in the case of $10^{\circ}$ broadening.

\section{Model-Independent Probabilities from Chemical Shift Anisotropies}

The model-independent analysis can also be extended to non-uniaxial NMR interactions such as the chemical shift interaction. This is of interest, for instance, for the analysis of the ${ }^{31} \mathrm{P} \mathrm{CSA}$ of the phosphate group, where the rigid-lattice ${ }^{31} \mathrm{P}$ tensor orientation is well established. ${ }^{18}$ Since the chemical shift tensor is not axially symmetric ( $\eta$ $\neq 0$ ), the chemical shift frequency is expressed in the PAS as

$$
\bar{\delta}=\delta \frac{1}{2}\left\langle 3 \cos ^{2} \theta-1-\eta \sin ^{2} \theta \cos 2 \phi\right\rangle,
$$

where $\delta=\sigma_{z z}$ is the principal value of the largest magnitude, $\eta=\left|\sigma_{x x}-\sigma_{y y}\right| / \sigma_{z z}$, and $\theta$ and $\phi$ are the polar coordinates of the director in the chemical shift PAS. Consider the case where $\bar{\delta}$ and $\delta$ have opposite signs. The extreme distribution with the largest $I(\theta)$ for a given $\bar{\delta} / \delta$ consists of two sharp peaks, one at $\theta=\theta$ (in the plane of $\phi=0^{\circ}$ ) and the other at the chemical shift value furthest away from $\delta=\sigma_{z z}$, which is $\sigma_{x x}=-\frac{\delta}{2}(1+\eta)$. Then an equivalent form of equation (4.40) is found,

$$
\bar{\delta} / \delta=I_{\lim }(\theta) \cdot \frac{1}{2}\left(3 \cos ^{2} \theta-1-\eta \sin ^{2} \theta\right)+\left(1-I_{\lim }(\theta)\right) \cdot\left(-\frac{1}{2}(1+\eta)\right) .
$$

It yields the limit to the integral probability 


$$
I(\theta) \leq I_{\lim }(\theta)=\frac{\bar{\delta} / \delta+\frac{1}{2}(1+\eta)}{\frac{1}{2}\left(3 \cos ^{2} \theta+\eta-\eta \sin ^{2} \theta\right)}
$$

which reduces to equation (4.41) for $\eta=0$.

\section{References}

(1) Seelig, J. Biochim. Biophys. Acta 1978, 515, 105.

(2) Rose, M. E. Elementary Theory of Angular Momentum; Wiley, New York, 1957.

(3) Saupe, A.; Englert, G. Phys. Rev. Lett. 1963, 11, 462.

(4) Saupe, A. Molec. Cryst. 1966, 1, 527.

(5) Gennes, P. G. d. The Physics of Liquid Crystals; Clarendon Press, Oxford, 1974.

(6) Skarjune, R.; Oldfield, E. Biochemistry 1979, 18, 5903.

(7) Emsley, J. W.; Lindon, J. C. NMR Spectroscopy using Liquid Crystal Solvents; Pergamon Press, Oxford, 1975.

(8) Scherer, P. G.; Seelig, J. Biochemistry 1989, 28, 7723.

(9) Seelig, J.; Gally, H.-U.; Wohlgemuth, R. Biochim. Biophys. Acta 1977, 467, 109.

(10) Gally, H.; Niederberger, W.; Seelig, J. Biochemistry 1975, 14, 3647.

(11) Snyder, L. C. J. Chem. Phys. 1965, 43, 4041.

(12) Hentschel, R.; Sillescu, H.; Spiess, H. W. Polymer 1981, 22, 1516.

(13) Hong, M.; Schmidt-Rohr, K.; Pines, A. J. Am. Chem. Soc. 1995b, 117, 3310.

(14) Sanders, C. R. Biophys. J. 1993, 64, 171.

(15) Seelig, J.; Seelig, A. Q. Rev. Biophys. 1980, 13, 19.

(16) Fung, B. M.; Afzal, J.; Foss, T. L.; Chau, M. J. Chem. Phys. 1986, 85, 4808.

(17) Hong, M.; Schmidt-Rohr, K.; Nanz, D. Biophys. J. 1995a, 69, 1939.

(18) Kohler, S. J.; Klein, M. P. Biochemistry 1976, 15, 968. 


\section{CHAPTER 5}

\section{NMR MEASUREMENTS OF PHOSPHOCHOLINE LIPIDS}

In this chapter, we present our experimental measurements of motionally-averaged NMR dipolar couplings and chemical shifts in $\mathrm{L}_{\alpha}$-phosphocholine. These NMR anisotropies are related to the orientation distributions of various molecular segments. Therefore they provide information on the dynamic conformation of the phosphocholine molecule in the liquid-cr;stalline phase.

\subsection{General Experimental Conditions}

\section{Materials}

Phosphatidylcholine was chosen for our studies for two reasons. First, it is one of the most abundant phospholipids in biological membranes. Second, ${ }^{2} \mathrm{H}$ quadrupolar couplings have been measured for more than 20 sites $^{1,2}$ in this molecule and can be used to compare with our dipolar coupling and chemical shift results.

Most experiments were conducted on two types of phosphatidylcholine lipids with different acyl chain compositions. Natural egg-yolk $L_{\alpha}$-lecithin with $99 \%$ purity was used in many dipolar coupling measurements, including directly-bonded $\mathrm{C}-\mathrm{H}$ couplings, $\mathrm{P}-\mathrm{H}$, $\mathrm{P}-\mathrm{C}$ and $\mathrm{H}-\mathrm{H}$ dipolar couplings. The acyl chains of egg-yolk lecithin are a mixture of 16:0 (34\%), 18:1 (31\%), 18:2 (18\%) and 18:0 (11\%) fatty acids. For the P-H/31 P CSA correlation experiment, egg phosphatidylethanolamine and sphingomyelin with $99 \%$ purity were also used. These samples were purchased as dry powders from Avanti Polar Lipids (Alabaster, AL).

The second phosphocholine sample used was synthetic dimyristoylphosphatidylcholine (DMPC). Unlabeled powder DMPC was purchased from Sigma (St. Louis, $\mathrm{MO}$ ) and used in ${ }^{13} \mathrm{C}$ WISE and C-H PDLF experiments. A synthetic ${ }^{13} \mathrm{C}$-labeled DMPC 
sample, 1-myristoyl-2-(1-13C-)myristoyl-sn-glycero-3-phosphatidylcholine, was prepared by $\mathrm{H}$. Zimmermann and used in the measurement of long-range $\mathrm{C}-\mathrm{H}$ dipolar couplings and segmental C-C dipolar couplings. The synthesis of this ${ }^{13} \mathrm{C}$-labeled sample is described as follows.

${ }^{1-13} \mathrm{C}$-myristic acid $\left({ }^{13} \mathrm{C}=99 \%\right)$ was prepared by low temperature carboxylation ($30^{\circ} \mathrm{C}$ ) of the tridecylbromide-Grignard in ether with ${ }^{13} \mathrm{CO}_{2}$. The corresponding labeled anhydride was prepared via the $1-13 \mathrm{C}$-myristic acid by using dicyclohexylcarbodiimide. ${ }^{3}$ Next, 1-myristoyl-2-(1-13C-)myristoyl-sn-glycero-3-phosphatidylcholine was synthesized by esterification 4,5 of 1-myristoyl-2-hydroxy-sn-glycero-3-phosphocholine (Avanti Polar Lipids) with the labeled myristic anhydride and dimethylaminopyridine in $\mathrm{CHCl}_{3}$. The reaction was monitored with TLC until the 2-lysophosphatidylcholine had disappeared. The product was purified by chromatography on Sephadex $\mathrm{LH}-20$ with $\mathrm{CHCl}_{3}$ as eluent. Most of the solvent evaporated at room temperature, and the labeled dimyristoylphosphatidylcholine was precipitated with ether. Repeated recrystallizations from $\mathrm{CHCl}_{3}$ / ether resulted in a white crystalline product (one spot on $\mathrm{TLC}=\mathrm{CHCl}_{3} / \mathrm{CH}_{3} \mathrm{OH} / \mathrm{H}_{2} \mathrm{O}=$ 65:25:10; iodine).

\section{Sample Preparation}

The dry phosphocholine powders were hydrated with $\mathrm{D}_{2} \mathrm{O}$ at lipid-water weight ratios from 70:30 to 50:50. Even at low levels of hydration, the samples were tested by ${ }^{31} \mathrm{P}$ and ${ }^{1} \mathrm{H}$ chemical shift spectra to be in the liquid-crystalline $\mathrm{L}_{\alpha}$ phase. A dry phospholipid powder was mixed with water mechanically, then the mixture was subjected to several freeze-thawing cycles with liquid nitrogen as the freezing agent. The procedure was repeated until a uniform aqueous dispersion, opaque in color, was obtained. The hydrated sample was inserted into a rotor or a NMR tube immediately after the last round of freezing in the liquid nitrogen, so that it could be packed easily and tightly in the container. The NMR tubes were sealed with Teflon tapes to avoid sample drying over time. For the 
rotors, however, special precaution was taken not only to avoid loss of water, but also to prevent the "mushy" sample from spilling out during spinning due to strong centrifugal forces. This was achieved by gluing the rotor cap to the rotor body with superglue. Other sealing media such as Teflon tapes or caps with an outgassing hole were found to be inadequate for preventing spilling, even when only moderate spinning speeds $(\sim 2 \mathrm{kHz})$ were used.

\section{NMR Measurements}

Two spectrometers were used for the experiments: a home-built $300 \mathrm{MHz}(7.07 \mathrm{~T})$ spectrometer (" $\varepsilon ")$ and a Bruker AM400 (9.4 T) spectrometer. The experiments with sample spinnning were carried out on the former, which is a solid-state spectrometer equipped with a Tecmag pulse programmer and data acquisition system. The sample was spun using a Doty Scientific $7 \mathrm{~mm}$ spinner in a home-built switching-angle spinning probehead. ${ }^{6}$ The spinning speed was approximately $2-3 \mathrm{kHz}$. The orientation of the rotor axis was controlled by a stepping motor attached to the bottom of the probe and a computerized motor controller with an angular precision of $0.1^{\circ}$. The rotor axis was hopped between two angles within less than $60 \mathrm{~ms}$.

Radiofrequency field strengths were chosen to be low to avoid overheating the sample. The ${ }^{1} \mathrm{H}$ rf fields varied between approximately $30 \mathrm{kHz}$ and $50 \mathrm{kHz}$, corresponding to $90^{\circ}$ pulse lengths of $5-8 \mu \mathrm{s}$. The ${ }^{13} \mathrm{C}$ rf fields were slightly higher, between $35 \mathrm{kHz}$ and $50 \mathrm{kHz}$. These rf field strengths were measured when the rotor axis was at the magic angle orientation. Since the $\mathrm{rf}$ coil in the probehead is wrapped around the stator body, the rf field strengths change with the rotor orientations.

The static $2 \mathrm{D}$ correlation experiments were conducted on the Bruker spectrometer with an inverse solution probe. The ${ }^{1} \mathrm{H}$ line of $\mathrm{H}_{2} \mathrm{O}$ was shimmed to about $6 \mathrm{~Hz}$. Proton $90^{\circ}$ pulse lengths of $13 \pm 2 \mu \mathrm{s}$, corresponding to rf fields of about $20 \mathrm{kHz}$, were used. MREV -8 cycle times varied from $180 \mu$ s to $200 \mu \mathrm{s}$. The MREV-8 scaling factor for these 
static experiments was tested experimentally to be $0.47 \pm 0.04$. CP Hartman-Hahn match was achieved at $B_{1}$ field strengths of about $(2 \pi \cdot 17) / \gamma \mathrm{kHz}$. The phospholipid samples packed in the $5 \mathrm{~mm}$ NMR tubes were found to be partially oriented with the directors preferentially perpendicular to the magnetic field axis. This probably resulted from the procedure of rolling the samples before inserting them into the NMR tubes, since rolling effectively orients the bilayer planes to be normal to the cylindrical axis. The PE and SPM samples were heated to $305 \mathrm{~K}$ and $320 \mathrm{~K}$ in order to reach the $\mathrm{L}_{\alpha}$ phase.

We found that the applied rf power was a sensitive experimental parameter, as the phosphocholine lipids undergo $\mathrm{L}_{\alpha}$-isotropic transitions near the room temperature. Moreover, If heating could cause slow deterioration of the samples by cleaving the ester linkages between the acyl chains and the glycerol, thereby producing free fatty acids over time. A spectroscopic indication of such sample deterioration is an extra carboxyl signal near $174 \mathrm{ppm}$, which can be distinguished from the normal carboxyl peak, in the ${ }^{13} \mathrm{C}$ MAS spectrum. Due to these considerations, moderate if powers and the shortest necessary irradiation times were used in the experiments.

An opposite problem was encountered sometimes: the sample temperature could be below the gel- $\mathrm{L}_{\alpha}$ phase transition temperature $\left(T_{m}\right)$. For example, $T_{m}$ is $23^{\circ} \mathrm{C}$ for DMPC. With the Bruker probe, the problem was avoided by turning on the heater attached to the probe. With the home-built SAS probe, which has no in-built heater and thermocouple in the probehead, sufficient heat could be produced by rf pulses to induce the phase transition. In addition, a heater was occasionally connected to the bearing air line to warm up the bearing air, which keeps the rotor afloat for spinning. The phase of the phospholipids was monitored by ${ }^{1} \mathrm{H},{ }^{31} \mathrm{P}$ and ${ }^{13} \mathrm{C}$ chemical shift spectra. The ${ }^{1} \mathrm{H}$ MAS linewidths and the ${ }^{31} \mathrm{P}$ static chemical shift anisotropies are sensitive indicators of the phase of phospholipids, whereas the ${ }^{13} \mathrm{C} \mathrm{CP}$ intensities indicate whether any transition to the isotropic phase has occurred. 


\subsection{Short-Range ${ }^{13} \mathrm{C}-1 \mathrm{H}$ Dipolar Couplings}

Directly-bonded ${ }^{13} \mathrm{C}-{ }^{1} \mathrm{H}$ dipolar couplings, including both their magnitudes and their signs, are obtained for all sites in lecithin. These directly-bonded (i.e. short-range) $\mathrm{C}-\mathrm{H}$ dipolar couplings provide the order parameters of the $\mathrm{C}-\mathrm{H}$ bonds, and thus the bond orientations with respect to the director. Proton-detected local field spectroscopy is used in combination with switching-angle spinning (SAS) to obtain these couplings. The SASPDLF pulse sequence is shown in Figure 5.1. During the evolution period, the sample is spun about an axis inclined at an angle away from the magic angle $\left(\theta_{1} \neq 54.74^{\circ}\right)$. Offmagic-angle spinning (OMAS) is necessary for preserving the anisotropic dipolar interaction. The polarization is then transferred from ${ }^{1} \mathrm{H}$ to ${ }^{13} \mathrm{C}$ by cross polarization. A mixing period of about $50 \mathrm{~ms}$ ensues, during which the sample is hopped to the magic angle, $\theta_{2}=54.74^{\circ}$. Isotropic ${ }^{13} \mathrm{C}$ chemical shifts are then observed during the detection period.

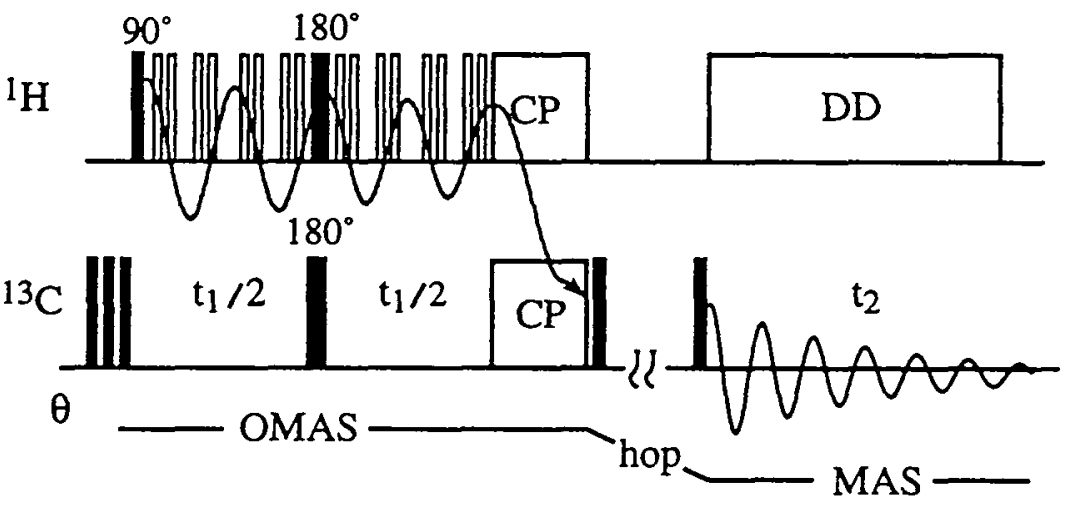

Figure 5.1 PDLF-SAS pulse sequence for measuring C-H dipolar couplings.

The resulting PDLF spectra of lecithin are shown in Figures 5.2. For clarity, the glycerol and headgroup spectral regions are shown separately from the acyl chain region. The spinning angles $\theta_{1}$ of these spectra are $63^{\circ}$ and $47^{\circ}$, corresponding to scaling factors $P_{2}\left(\cos \theta_{1}\right)$ of -0.2 and +0.2 . The CP contact time used is $0.5 \mathrm{~ms}$. 
(a)

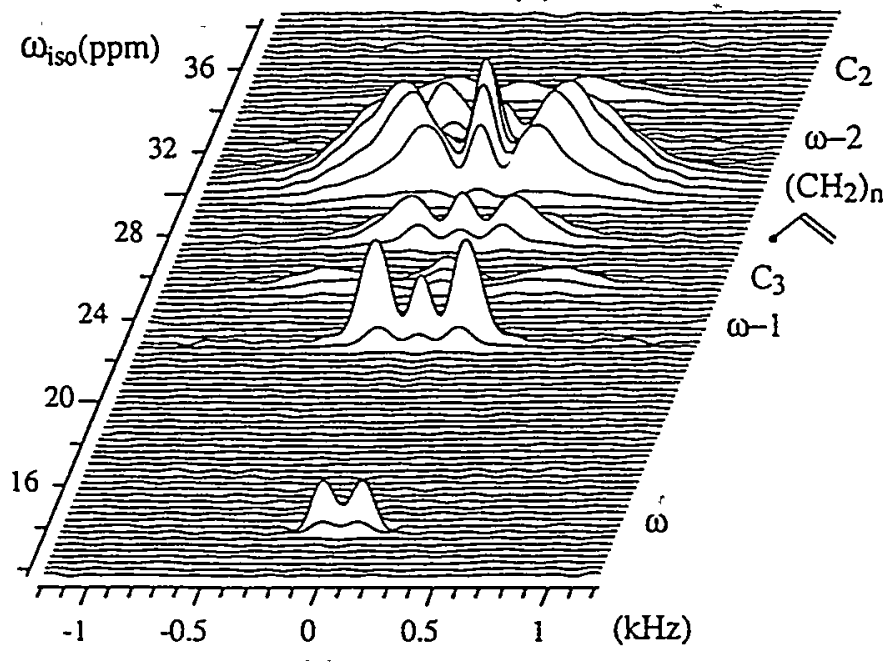

(b)

(c)

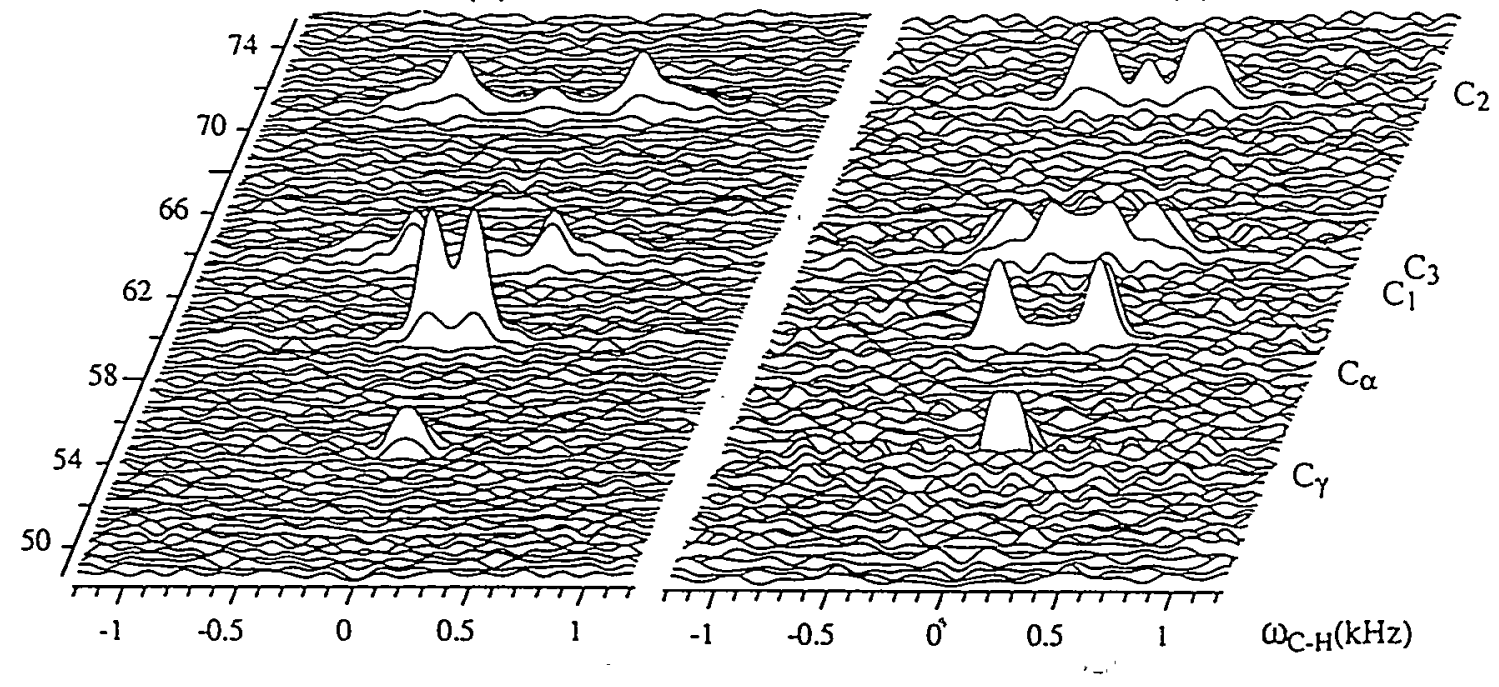

Figure 5.2 $\mathrm{L}_{\alpha}$-lecithin $\mathrm{C}-\mathrm{H}$ PDLF spectra obtained under SAS. (a) Acyl chain region. (b) Headgroup and glycerol regions. Spinning scaling factor in $\omega_{1}$ is $P_{2}\left(\cos 63^{\circ}\right.$ ) $=-0.2$. (c) Headgroup and glycerol regions, with $P_{2}\left(\cos 47^{\circ}\right)=+0.2$ in $\omega_{1}$. 
In the 2D spectra, $\mathrm{C}-\mathrm{H}$ dipolar Pake patterns with splittings of $200-1300 \mathrm{~Hz}$ are observed in the $\omega_{1}$ dimension. They are separated along the $\omega_{2}$ dimension according to the isotropic ${ }^{13} \mathrm{C}$ chemical shifts of the various sites. To a first approximation, these splittings correspond to the motionally-averaged $\mathrm{C}-\mathrm{H}$ dipolar couplings $\bar{\delta}_{\mathrm{CH}}$. Inversion of the spinning scaling factor from -0.2 to +0.2 reduces the $\mathrm{C}-\mathrm{H}$ splittings of most segments except for the headgroup $\mathrm{C} \alpha$ site, which shows a pronounced increase in the $\mathrm{C}-\mathrm{H}$ splitting.

The change in the magnitudes of the $\mathrm{C}-\mathrm{H}$ splittings with the spinning scaling factor results from the fact that isotropic $\mathrm{C}-\mathrm{H} J$ couplings also contribute to the observed spectral splittings. However, unlike the anisotropic dipolar coupling, the scalar coupling is unaffected by sample spinning. Since the dipolar coupling $\bar{\delta}_{C H}$ of the segments can be either positive or negative, whereas the one-bond C-H scalar coupling $2 \pi^{1} J_{C H}$ is always positive, the observed spectral splittings $\omega_{\text {split }}$ are

$$
\left|\bar{\delta}_{C H} \cdot P_{2}\left(\cos \theta_{1}\right) \pm 2 \pi^{1} J_{C H}\right|=\omega_{\text {split }}
$$

where the absolute bar results from the symmetry of the dipolar spectra. A detailed analysis of various combinations of the dipolar and scalar couplings, taking into account their relative signs and magnitudes, is illustrated in Figure 5.3. It shows that when the two $\mathrm{C}-\mathrm{H}$ splittings of a site both exceed twice the value of $2 \pi^{1} J_{C H}$, then the average of the two splittings corresponds to the magnitude of the dipolar coupling $\left|\bar{\delta}_{C H}\right|$, and the difference gives the $J$ coupling. Most methylene groups in lecithin, except for those at the ends of the acyl chains and headgroup, have sufficiently large dipolar couplings so that the above condition applies. If $\omega_{\text {split }} \leq 2 \cdot 2 \pi^{1} J_{C H}$, then the difference of the two splittings gives $\left|\bar{\delta}_{C H}\right|$, while the average is the $J$ coupling. Figure 5.3 also illustrates how the sign of dipolar coupling relative to scalar coupling can be determined from two spectra with 

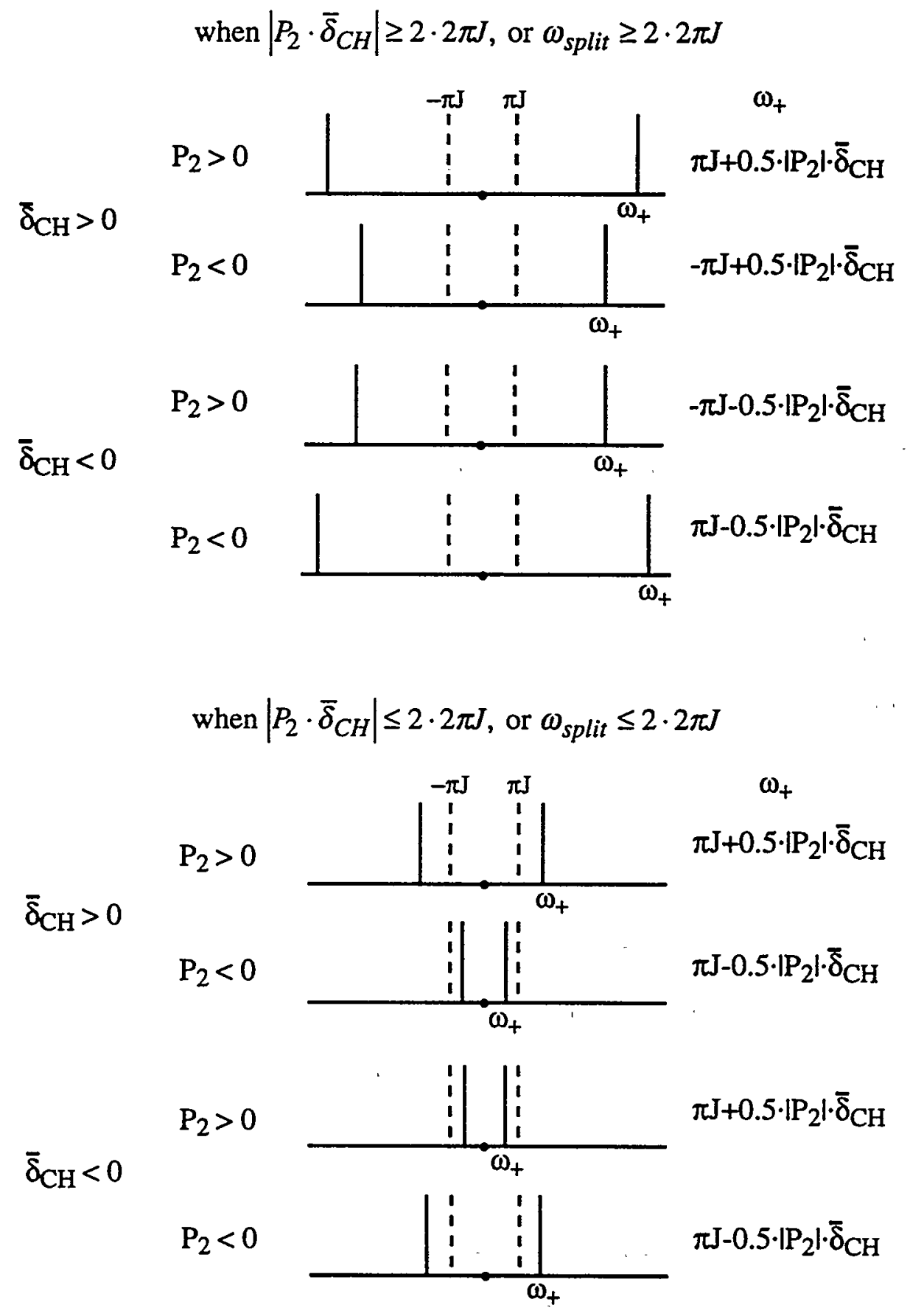

Figure 5.3 Analysis of the signs and magnitudes of dipolar couplings. Depending on the relative sign of the dipolar coupling with the $J$ coupling, the spectral splitting increases or decreases when the OMAS spinning angle is changed. 
opposite $P_{2}$ values. The dipolar coupling is positive if the spectral splitting increases from the spectrum with a negative $P_{2}$ to that with a positive $P_{2}$, and vice versa.

From the averaged $\mathrm{C}-\mathrm{H}$ dipolar coupling $\bar{\delta}_{\mathrm{CH}}$, we can calculate the $\mathrm{C}-\mathrm{H}$ bond order parameter $S_{C H}$ by dividing $\bar{\delta}_{C H}$ with the corresponding rigid-lattice coupling $\delta_{C H}$.

In the spectra of Figure 5.2(b-c), the $\mathrm{C}-\mathrm{H}$ splittings of the headgroup $\beta$ and $\gamma$ sites have relatively low sensitivities. This can be attributed to inefficient cross polarization in these segments, as they are more mobile than the glycerol and the $\mathrm{C} \alpha$ sites. The sensitivities of these weakly-coupled $\mathrm{C}-\mathrm{H}$ segments can be enhanced by increasing the $\mathrm{CP}$ contact time. Thus two additional PDLF experiments are performed, in which the CP contact times are extended to $2 \mathrm{~ms}$ from $0.5 \mathrm{~ms}$. Synthetic DMPC is used instead of eggyolk lecithin. The sample is spun off the magic angle with scaling factors of $P_{2}= \pm 0.2$ during both $t_{1}$ and $t_{2}$ periods, in order to avoid the mechanical strain of hopping. The resolution of the ${ }^{13} \mathrm{C}$ chemical shift dimension is still sufficient at these spinning scaling factors.

The resulting OMAS-PDLF spectra of DMPC in the headgroup and glycerol regions are shown in Figure 5.4. Strong $C \beta$ and $C \gamma$ dipolar spectra with high resolution can be observed in the dipolar dimension. These dipolar patterns exhibit higher resolution than in the SAS-PDLF spectra (Figure 5.2) because powder broadening is partially removed by "spreading-out" the chemical shift frequencies in the ${ }^{13} \mathrm{C}$ dimension as a result of off-magic-angle spinning. When the spinning scaling factor changes from -0.2 (Figure 5.4a) to +0.2 (Figure 5.4b), the $\mathrm{C} \gamma-\mathrm{H} \gamma$ and $\mathrm{C} \alpha-\mathrm{H} \alpha$ splittings increase whereas the $\mathrm{C} \beta$ $\mathrm{H} \beta$ splitting decreases. According to the combinations of dipolar and scalar couplings shown in Figure 5.3, these indicate that the $\gamma$ methyl groups have a positive $\mathrm{C}-\mathrm{H}$ order parameter while the $\beta$ group has a negative one. In addition, the magnitude of the dipolar coupling of each site is equal to the difference between the two splittings, which are smaller than $2 \cdot 2 \pi J \approx 250 \mathrm{~Hz}$ due to the large mobilities of each segment. The resulting $\mathrm{C} \beta-\mathrm{H} \beta$ coupling is calculated to be $+175 \mathrm{~Hz}$ and the $\mathrm{C} \gamma-\mathrm{H} \gamma$ coupling about $-110 \mathrm{~Hz}$. These 
values deviate somewhat from those obtained in lecithin using the SAS-PDLF technique. This is most likely due to the relatively low hydration levels of the lecithin sample compared to the DMPC sample. A previous ${ }^{2} \mathrm{H}$ NMR study has found hydration dependence in the headgroup $\mathrm{C}-\mathrm{H}$ order parameters. ${ }^{7}$

Now we present the $\mathrm{C}-\mathrm{H}$ dipolar couplings of the double-bond segments in the acyl chains. In the SAS-PDLF spectrum of lecithin taken at tine spinning angle of $63^{\circ}\left(P_{2}=\right.$ -0.2 ), three $\mathrm{C}-\mathrm{H}$ couplings can be resolved in the double-bond region (Figure 5.5). The two splittings at 130.2 ppm are assigned to the $\mathrm{C} 9$ and $\mathrm{C} 13$ segments, ${ }^{8}$ where $\mathrm{C} 13$ belongs to the second double bond in the 9,12-linoleic acid chains, and the small splitting at 128.2 ppm ${ }^{13} \mathrm{C}$ chemical shift corresponds to the $\mathrm{C} 10$ site. From two PDLF spectra obtained with the dipolar scaling factors of $-0.2\left(\theta_{1}=63^{\circ}\right)$ and $+0.2\left(\theta_{1}=47^{\circ}\right)$, the average $\mathrm{C}-\mathrm{H}$ couplings are found to be $+2.1 \mathrm{kHz}$ for the $\mathrm{C} 9$ site and $+0.83 \mathrm{kHz}$ for the $\mathrm{C} 10$ site, which correspond to $\mathrm{C}-\mathrm{H}$ bond order parameters of -0.09 and -0.036 , respectively. The $\mathrm{C}-\mathrm{H}$ coupling for the C13 site could not be obtained due to its low resolution in the $P_{2}=+0.2$ spectrum (not shown). These dipolar couplings can be compared with the ${ }^{2} \mathrm{H}$ NMR results, ${ }^{9}$ where $\mathrm{C}-\mathrm{H}$ bond order parameters of -0.102 and +0.019 were found for the $\mathrm{C} 9$ and $\mathrm{C} 10$ sites in the cis-9-oleic acid chain. The discrepancy in the $\mathrm{C} 10$ coupling sign is discussed in the next chapter. 


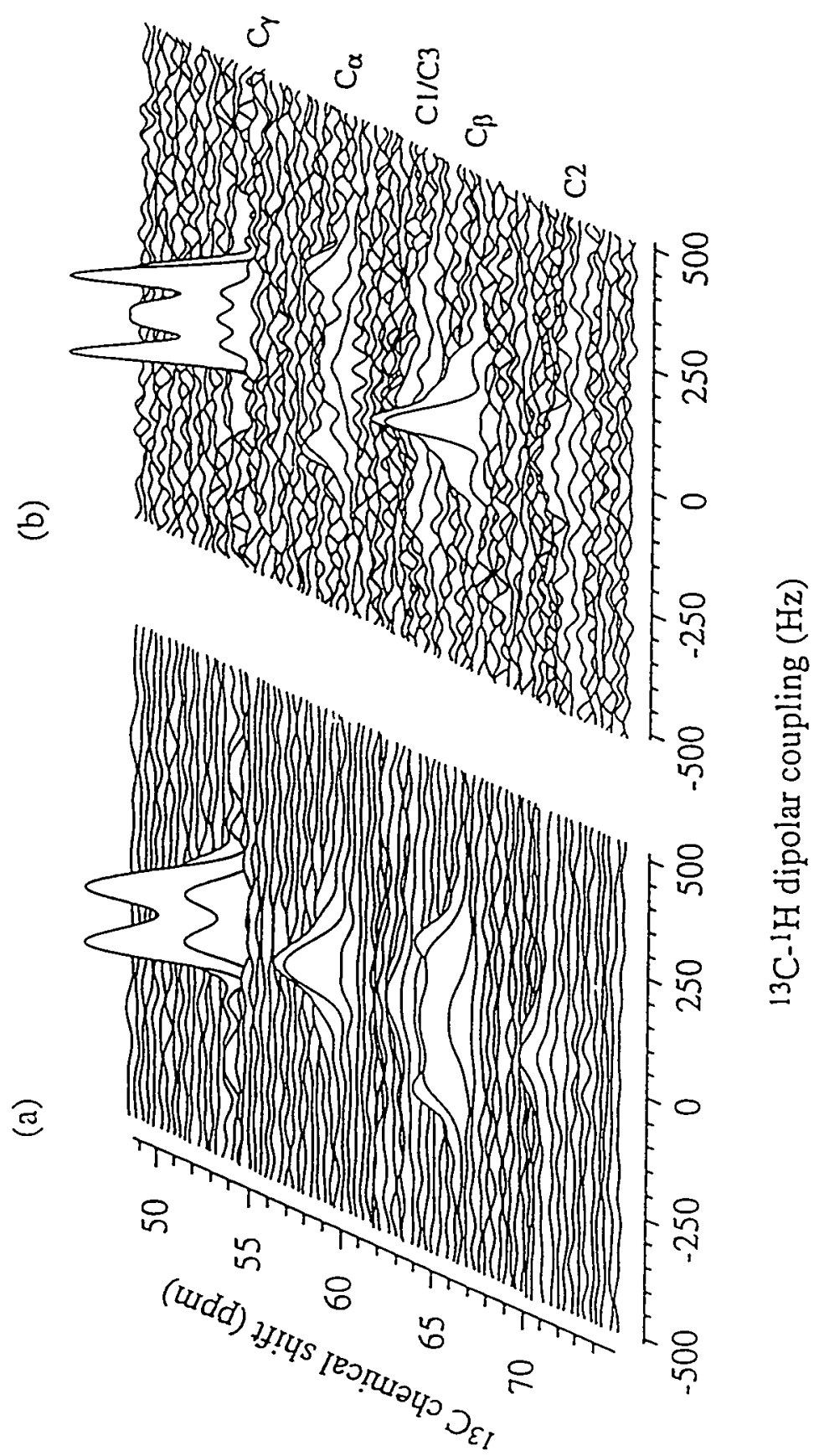

Figure 5.4 Headgroup and glycerol regions of $\mathrm{L}_{\alpha}$-DMPC C-H PDLF spectra obtained under OMAS. The dipolar couplings in $\omega_{1}$ are scaled by (a) $P_{2}\left(\cos 63^{\circ}\right)=-0.2$ and (b) $P_{2}\left(\cos 47^{\circ}\right)=+0.2$. CP contact times: $2 \mathrm{~ms}$. Measuring times: 8 and 10 hours, respectively. 
(a)

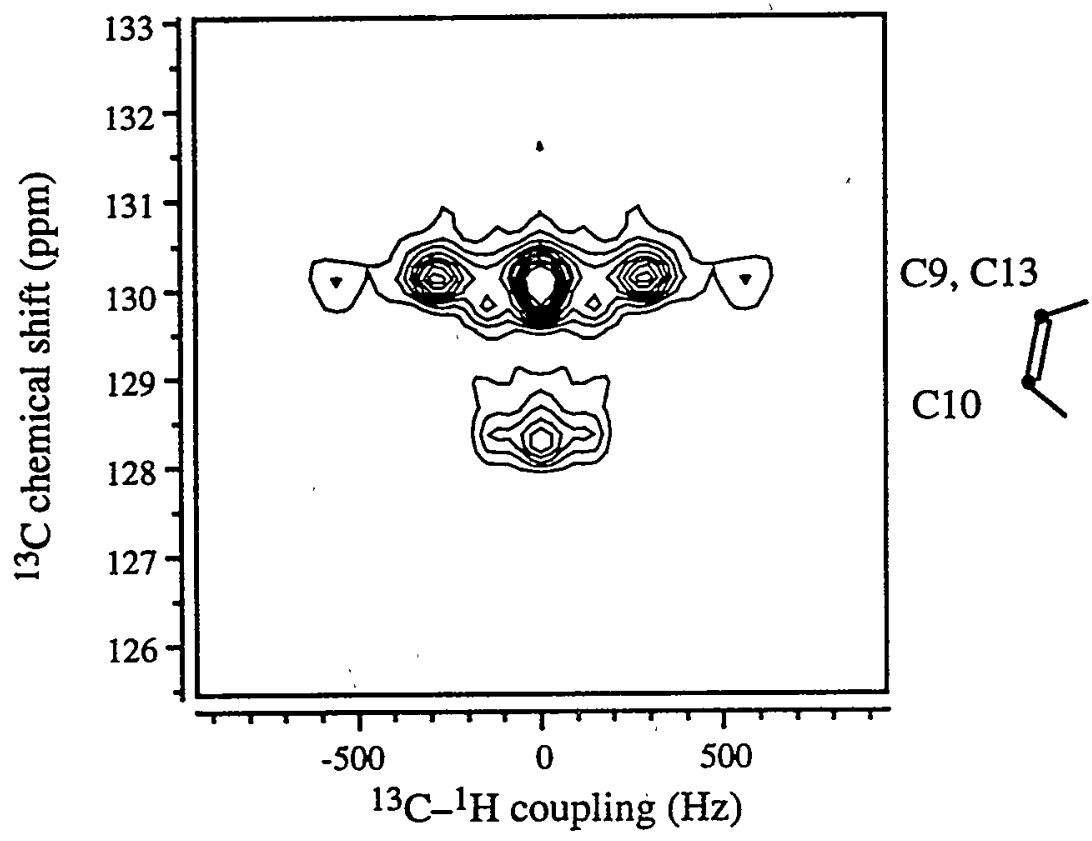

(b)

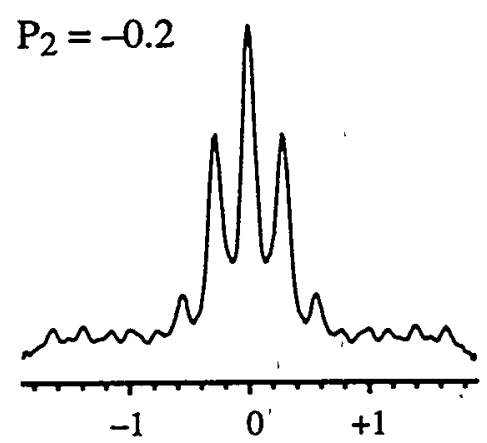

${ }^{13} \mathrm{C}-{ }^{1} \mathrm{H}$ coupling $(\mathrm{kHz})$ (c)

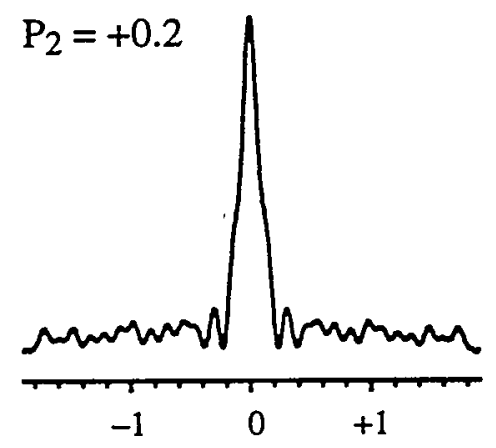




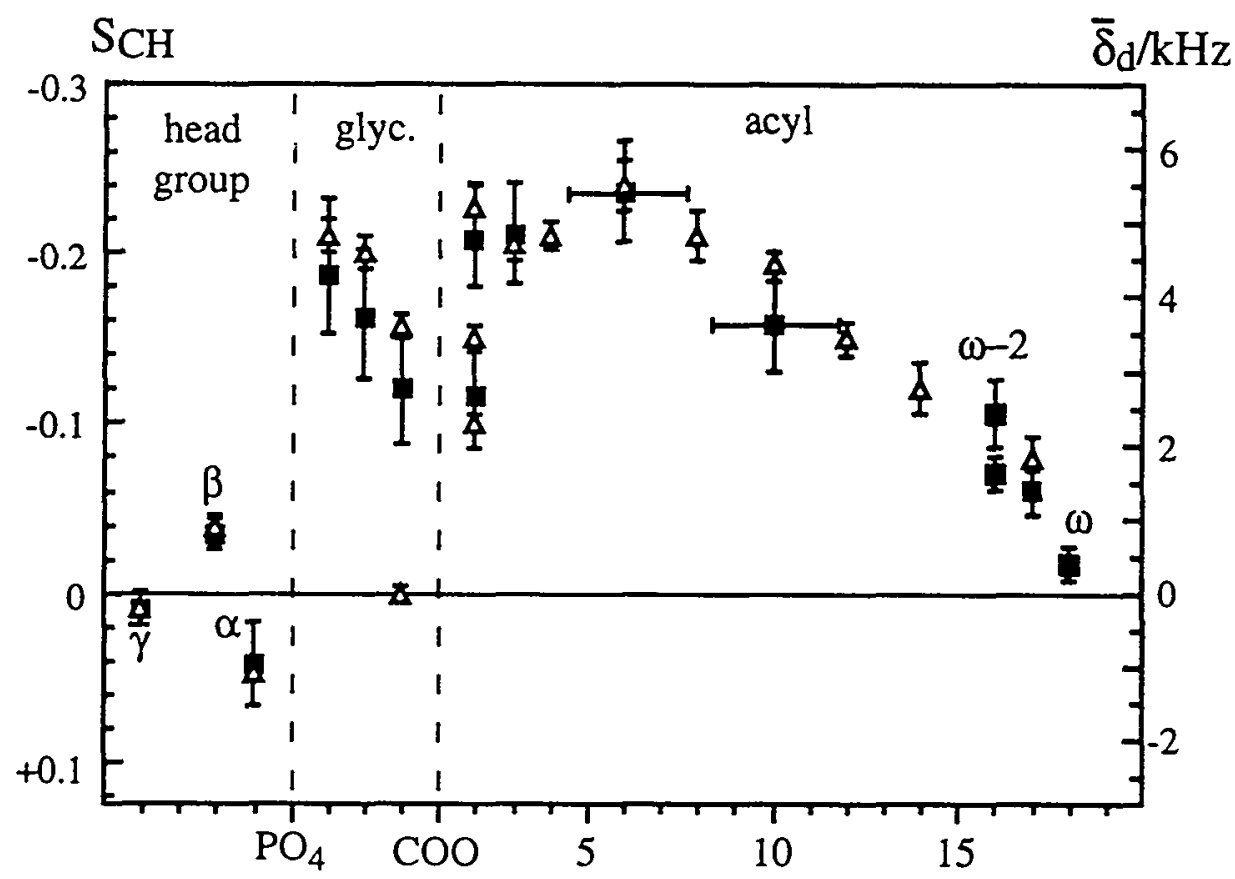

Figure 5.6 Averaged $\mathrm{C}-\mathrm{H}$ dipolar couplings and the corresponding order parameters in $\mathrm{L}_{\alpha}$-lecithin. The dipolar order parameters $(\boldsymbol{m})$ are compared with ${ }^{2} \mathrm{H}$ order parameters $(\Delta)$ from the literature.

In Figure 5.6, the directly-bonded $\mathrm{C}-\mathrm{H}$ dipolar couplings in phosphocholine lipids are summarized and compared with ${ }^{2} \mathrm{H}$ order parameters from the literature. ${ }^{1}$ The right ordinate indicates the motionally-averaged dipolar couplings, and the left ordinate the corresponding bond order parameters. The abscissa denotes the molecular segments, starting with the headgroup $\mathrm{C} \gamma$ site and ending at the acyl chain $\mathrm{C} \omega$ site. For the first time, the $\mathrm{C}-\mathrm{H}$ bond order parameters are determined completely, including their signs. These fully-determined $S_{C H}$ are important for testing structural models of the lipids ${ }^{10}$ and for solving segmental order tensors (chapter 6). ${ }^{9.11}$ In comparison, the $\mathrm{C}-\mathrm{H}$ bond order parameters from ${ }^{2} \mathrm{H}$ NMR are incomplete since only the absolute values of the ${ }^{2} \mathrm{H}$ quadrupolar couplings can be measured. In Figure 5.6, the ${ }^{2} \mathrm{H} S_{C H}$ are shown with the same signs as the dipolar couplings for clarity. Due to sample spinning and multiple-pulse 
homonuclear decoupling, the experimental $\mathrm{C}-\mathrm{H}$ dipolar couplings are scaled to approximately $10 \%(0.2 \times 0.5)$ of the full motionally-averaged couplings. Therefore, the dipole bond order parameters are less precise than the ${ }^{2} \mathrm{H}$ order parameters.

The reproducibility of the acyl chain $S_{C H}$ between lecithin and DMPC indicates that the acyl chain composition does not affect the $\mathrm{C}-\mathrm{H}$ dipolar couplings significantly, as concluded also by the ${ }^{2} \mathrm{H}$ NMR studies. ${ }^{1,9,12-15}$ The exceptions to this may be the doublebond segments and the acyl chain ends.

\subsection{Long-Range ${ }^{13} \mathrm{C}-{ }^{1} \mathrm{H}$ Dipolar Couplings}

In order to obtain constraints on the headgroup and $s n-2$ chain orientations, longrange ${ }^{13} \mathrm{C}-{ }^{1} \mathrm{H}$ dipolar couplings are measured in ${ }^{13} \mathrm{CO}$-labeled DMPC by ${ }^{13} \mathrm{C} /{ }^{1} \mathrm{H}$ heteronuclear correlation (HETCOR) experiments. ${ }^{16-18}$ As shown by the pulse sequence in Figure $5.7 \mathrm{a}, \mathrm{a}^{1} \mathrm{H} 90^{\circ}$ pulse excites the ${ }^{1} \mathrm{H}$ magnetization into the $x y$ plane of the rotating frame, where it evolves under the chemical shift interaction and the $\mathrm{C}-\mathrm{H}$ scalar coupling during $t_{1}$. At the end of the evolution period, the ${ }^{1} \mathrm{H}$ magnetization is transferred to the ${ }^{13} \mathrm{C}$ spins by cross polarization, and ${ }^{13} \mathrm{C}$ chemical shifts are detected during $t_{2}$. A CP contact time of $2 \mathrm{~ms}$ is used. The sample is spun at the magic angle with respect to the magnetic field throughout the experiment in order to remove all anisotropic interactions. This leaves the ${ }^{1} \mathrm{H}$ and ${ }^{13} \mathrm{C}$ isotropic chemical shifts to be correlated in the $2 \mathrm{D}$ spectrum, with additional $\mathrm{C}-\mathrm{H}$ scalar couplings in the ${ }^{1} \mathrm{H}$ dimension. The signal intensities reflect the efficiencies of polarization transfer from ${ }^{1} \mathrm{H}$ to ${ }^{13} \mathrm{C}$, which is a qualitative measure of the strengths of the $\mathrm{C}-\mathrm{H}$ dipolar couplings. The $99 \%$ enrichment of the ${ }^{13} \mathrm{CO} 2$ carbon facilitates the observation of resonances from protons that couple significantly to the ${ }^{13} \mathrm{CO} 2$ carbon. 
(a)

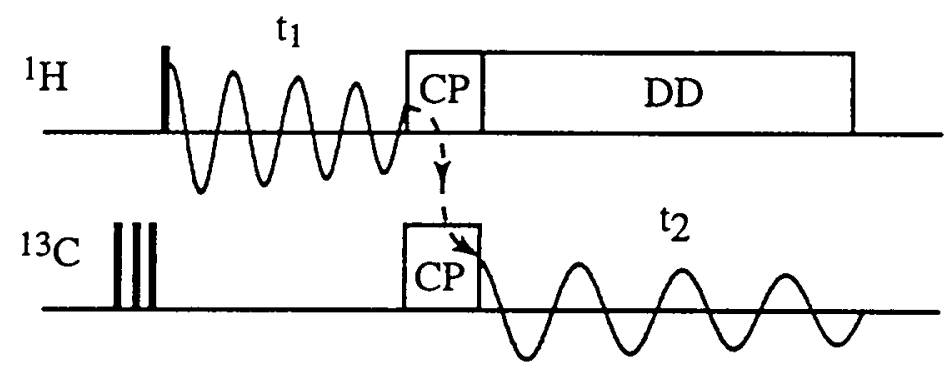

(b)

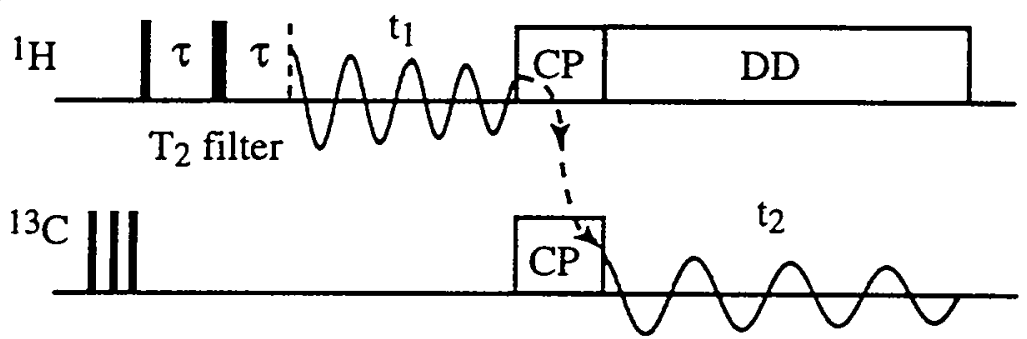

Figure $5.7{ }^{13} \mathrm{C} /{ }^{1} \mathrm{H}$ HETCOR pulse sequences. (a) Without a ${ }^{1} \mathrm{H} \mathrm{T}_{2}$ filter. (b) With ${ }^{1}{ }^{1} \mathrm{H}_{2}$ filter.

Figure 5.8 shows the ${ }^{13} \mathrm{C} /{ }^{1} \mathrm{H}$ HETCOR spectrum of the CO2-labeled DMPC. The acyl chain and headgroup segments, whose isotropic ${ }^{13} \mathrm{C}$ chemical shifts occur below 75 ppm, exhibit correlations between the directly-bonded protons and carbons. Due to C-H $J$ couplings, the ${ }^{1} \mathrm{H}$ resonances are split into doublets (or triplets), whose centers correspond to the ${ }^{1} \mathrm{H}$ isotropic shifts. Comparison with a $1 \mathrm{D}^{1} \mathrm{H}$ MAS spectrum of the same sample (Figure 5.9d) demonstrates that higher ${ }^{1} \mathrm{H}$ resolution is achieved in the 2D spectrum due to the additional separation in the ${ }^{13} \mathrm{C}$ dimension. In particular, the headgroup and glycerol ${ }^{1} \mathrm{H}$ signals, which overlap significantly in the $1 \mathrm{D}$ spectrum, are distinguishable in the $2 \mathrm{D}$ spectrum.

In the ${ }^{13} \mathrm{CO} 2$ cross section at $174.2 \mathrm{ppm}$, strong signals can be observed at several ${ }^{1} \mathrm{H}$ chemical shifts (Figure $5.9 \mathrm{a}$ ). These ${ }^{1} \mathrm{H}$ resonances are not split by $J$ couplings due to the lack of directly-bonded protons to the ${ }^{13} \mathrm{CO} 2$ carbon. The strongest ${ }^{1} \mathrm{H}$ signal occurs at $2.2 \mathrm{ppm}$ and results from the acyl $\mathrm{H} 2$ 's, which are the nearest protons to the ${ }^{13} \mathrm{CO} 2$ 
carbon. More downfield, ${ }^{1} \mathrm{H}$ signals are observed at the chemical shifts of $\mathrm{H} \gamma$ 's (3.3 ppm), the glycerol $\mathrm{H}_{\mathrm{G} 3}$ or the headgroup $\mathrm{H} \beta$ protons (3.9 ppm), and the $\mathrm{H}_{\mathrm{G} 1}$ or the $\mathrm{H} \alpha$ protons $(4.4 \mathrm{ppm})$. These multiple ${ }^{1} \mathrm{H}$ resonances indicate that ${ }^{13} \mathrm{CO} 2$ not only couples significantly to its nearest-neighbor protons, but also to protons as far as eleven bonds away, in the headgroup and glycerol segments.

The pattern of the strong $\mathrm{H} 2$ and the weak $\mathrm{H} 3$ signals in the ${ }^{13} \mathrm{CO} 2$ cross section shows that the acyl chain is not in its all-trans conformation at the $\mathrm{C} 2$ segment. Otherwise the coupling of the ${ }^{13} \mathrm{CO} 2$ carbon to the $\mathrm{H} 3$ protons would have been stronger than to the $\mathrm{H} 2$ protons due to both distance and orientation factors. The ${ }^{1} \mathrm{H}$ spectrum expected for an acyl chain with mostly trans conformations is provided experimentally by the ${ }^{13} \mathrm{C}$ cross section at $176.7 \mathrm{ppm}$ (Figure 5.9c). It corresponds to the carboxyl carbon in free myristic acid, which is present at a small concentration in an early batch of sample. The cross section shows that the $\mathrm{H} 3$ signal in the $\left(\mathrm{CH}_{2}\right)_{n}$ region is indeed more intense than the $\mathrm{H} 2$ signal.

The ${ }^{1} \mathrm{H}$ resonance assignment in the ${ }^{13} \mathrm{CO} 2$ cross section of the $2 \mathrm{D}$ HETCOR spectrum is complicated by the partial overlap of the headgroup and glycerol proton signals. To separate these two types of resonances, we exploit the ${ }^{1} \mathrm{H}$ relaxation times. In the $1 \mathrm{D}{ }^{1} \mathrm{H}$ MAS spectrum, the $\mathrm{H} \alpha$ and $\mathrm{H} \beta$ resonances are narrower than the glycerol $\mathrm{H}_{\mathrm{Gl}}$ and $\mathrm{H}_{\mathrm{G} 3}$ signals, suggesting longer spin-spin $\left(\mathrm{T}_{2}\right)$ relaxation times for the headgroup protons. This hypothesis is verified by a ${ }^{13} \mathrm{C}$ CPMAS experiment, in which a proton $\mathrm{T}_{2}$ relaxation filter of varying durations $(2 \tau)$ is inserted before the cross polarization step. The $\mathrm{T}_{2}$ filter consists of a delay $\tau$ before and after a $180^{\circ}$ pulse that refocuses the ${ }^{1} \mathrm{H}$ chemical shift. The resulting ${ }^{13} \mathrm{C}$ spectra (Figure 5.10) indicate that at pre-echo delays of $\tau \geq 100 \mu s$, the glycerol ${ }^{13} \mathrm{C}$ signals are mostly suppressed. Thus a proton $\mathrm{T}_{2}$ filter of 240 $\mu s$ is inserted before the ${ }^{1} \mathrm{H}$ chemical shift evolution in the HETCOR pulse sequence to filter out the glycerol ${ }^{1} \mathrm{H}$ resonances. 
(a)

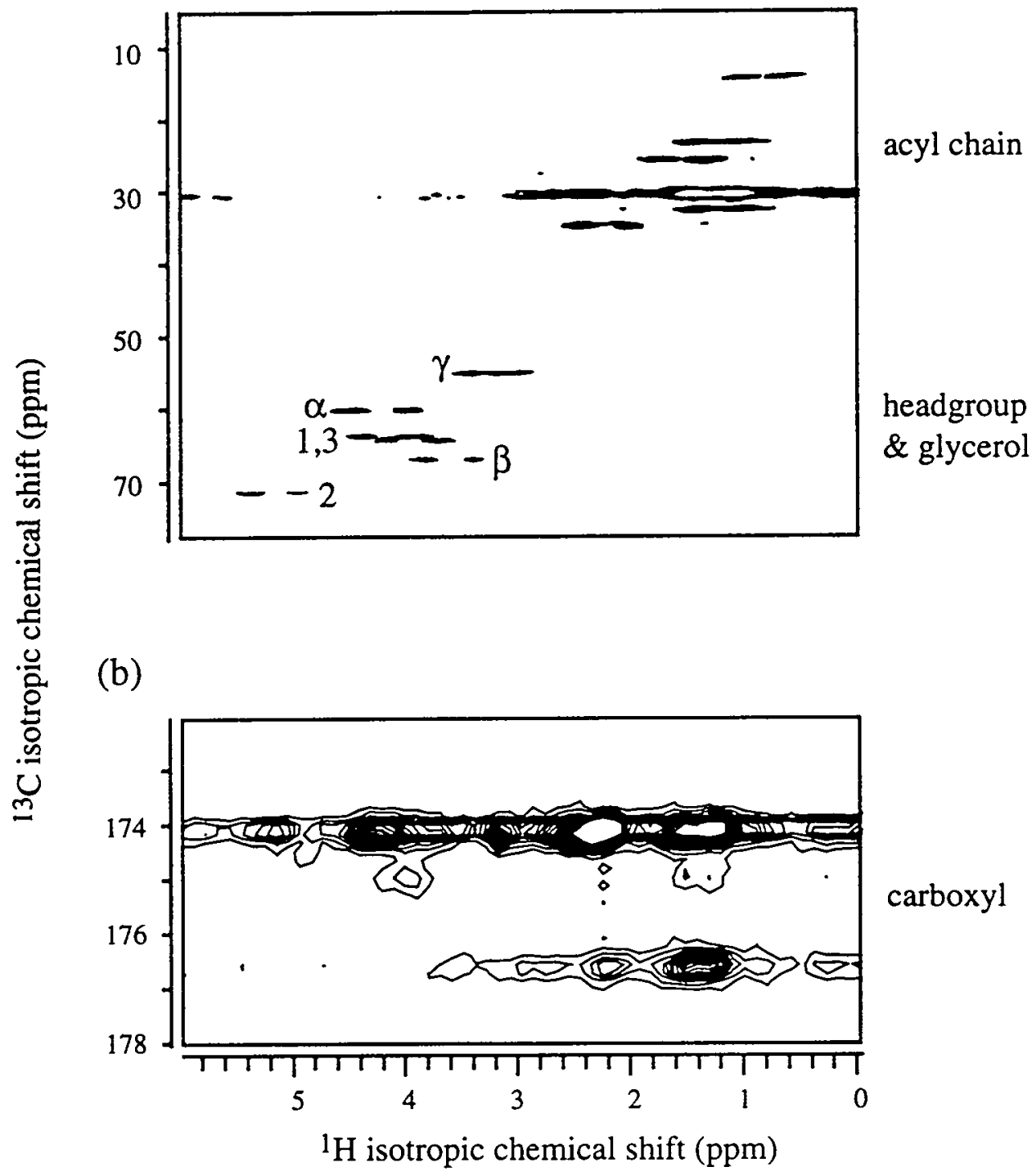

Figure 5.8 ${ }^{13} \mathrm{CO} 2-\mathrm{DMPC}$ HETCOR spectrum obtained without proton $\mathrm{T}_{2}$ filter. (a) Acyl chain, headgroup and glycerol regions. (b) Carboxyl region. CP contact time: 2 ms. Measuring time: 7 hours. 
(a)

(b)

(c)

(d)

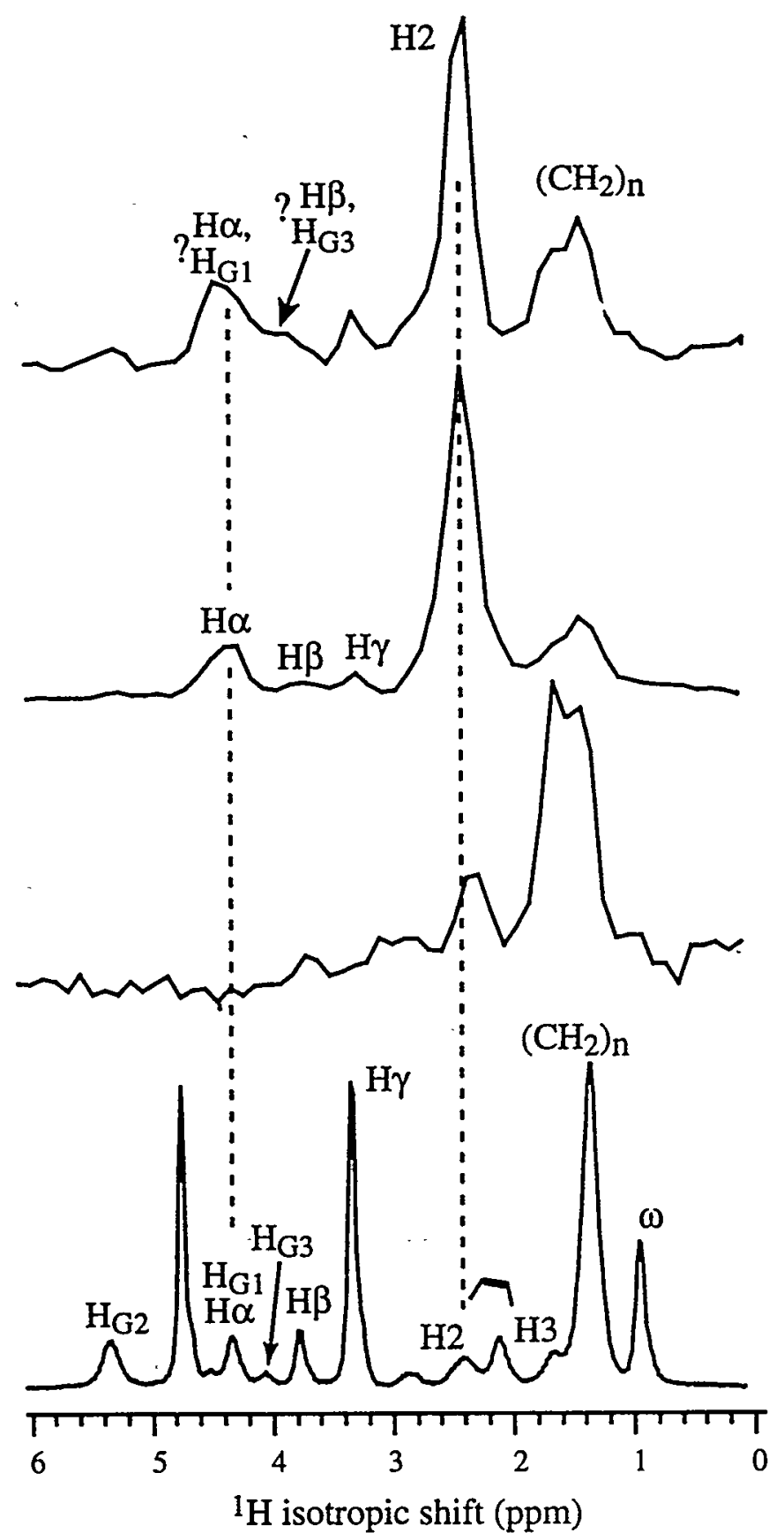

Figure 5.9 ${ }^{13} \mathrm{CO} 2$ cross sections from DMPC HETCOR spectra. (a) Without a ${ }^{1} \mathrm{H}$ $\mathrm{T}_{2}$ filter. (b) With a ${ }^{1} \mathrm{H} \mathrm{T}_{2}$ filter of $2 \tau=240 \mu \mathrm{s}$. (c) ${ }^{13} \mathrm{CO} 2$ cross section of free myristic acid, without a $\mathrm{T}_{2}$ filter. (d) DMPC ${ }^{1} \mathrm{H}$ MAS spectrum for peak assignment. 
The ${ }^{13} \mathrm{CO} 2$ cross section from the ${ }^{1} \mathrm{H}$-filtered HETCOR spectrum is displayed in Figure 5.9b. Clearly, the number and the intensities of the ${ }^{1} \mathrm{H}$ resonances between 3.5 $\mathrm{ppm}$ and $5 \mathrm{ppm}$ are substantially reduced. Only two peaks are largely retained, and they can be assigned to the $\mathrm{H} \alpha(4.4 \mathrm{ppm})$ and $\mathrm{H} \beta(3.8 \mathrm{ppm})$ protons unambiguously. The $\mathrm{H} \alpha$ signal is particularly strong, indicating appreciable $\mathrm{CO} 2-\mathrm{H} \alpha$ couplings. In contrast, the $\mathrm{H} \beta$ and $\mathrm{H} \gamma$ signals are much weaker. These features suggest that the $\mathrm{CO} 2$ carbon in phosphocholine couples to the headgroup protons, and mostly significantly to the $\mathrm{H} \alpha$ protons.

The different ${ }^{1} \mathrm{H}$ resonance intensities between the $\mathrm{CO} 2$ cross section of the $\mathrm{T}_{2}$ filtered HETCOR spectrum and the 1D MAS spectrum provide qualitative information on the relative strengths of the various ${ }^{13} \mathrm{CO} 2-{ }^{1} \mathrm{H}$ dipolar couplings. However, two conditions must be satisfied for correlating the $\mathrm{CP}$ intensities with the dipolar coupling strengths. First, ${ }^{1} \mathrm{H}$ spin diffusion must be negligible during the $\mathrm{CP}$ contact time. The absence of significant spin diffusion between the headgroup and the glycerol protons during the $2 \mathrm{~ms}$ contact time in our experiments is proved by the ${ }^{13} \mathrm{C}$ CPMAS spectrum with $2 \tau=240 \mu \mathrm{s}$ (Figure 5.10). If ${ }^{1} \mathrm{H}$ spin diffusion from the headgroup to the glycerol backbone occurred, the glycerol ${ }^{13} \mathrm{C}$ peaks would be observed, which is not the case. In addition, our ${ }^{1} \mathrm{H}$ WISE experiments with sample hopping (Figure 5.17) indicate that little spin diffusion occurs within $30 \mathrm{~ms}$. The weak spin diffusion in $\mathrm{L}_{\alpha}$-phosphocholine bilayers is not unexpected, since intramolecular $\mathrm{H}-\mathrm{H}$ dipolar couplings are significantly averaged by the fast uniaxial motion of the molecules, while intermolecular $\mathrm{H}-\mathrm{H}$ and $\mathrm{C}-\mathrm{H}$ couplings are averaged out by the rapid lateral diffusion of the molecules in the bilayer plane. ${ }^{19,20}$

The second requirement for correlating the $\mathrm{CP}$ intensities with the dipolar coupling strengths is that the CP contact time must be chosen within the initial increase of the polarization transfer curve. This ensures that the relative intensities of the $\mathrm{CP}$ signal to the direct polarization signal corresponds to the dipolar coupling strength. For expected 


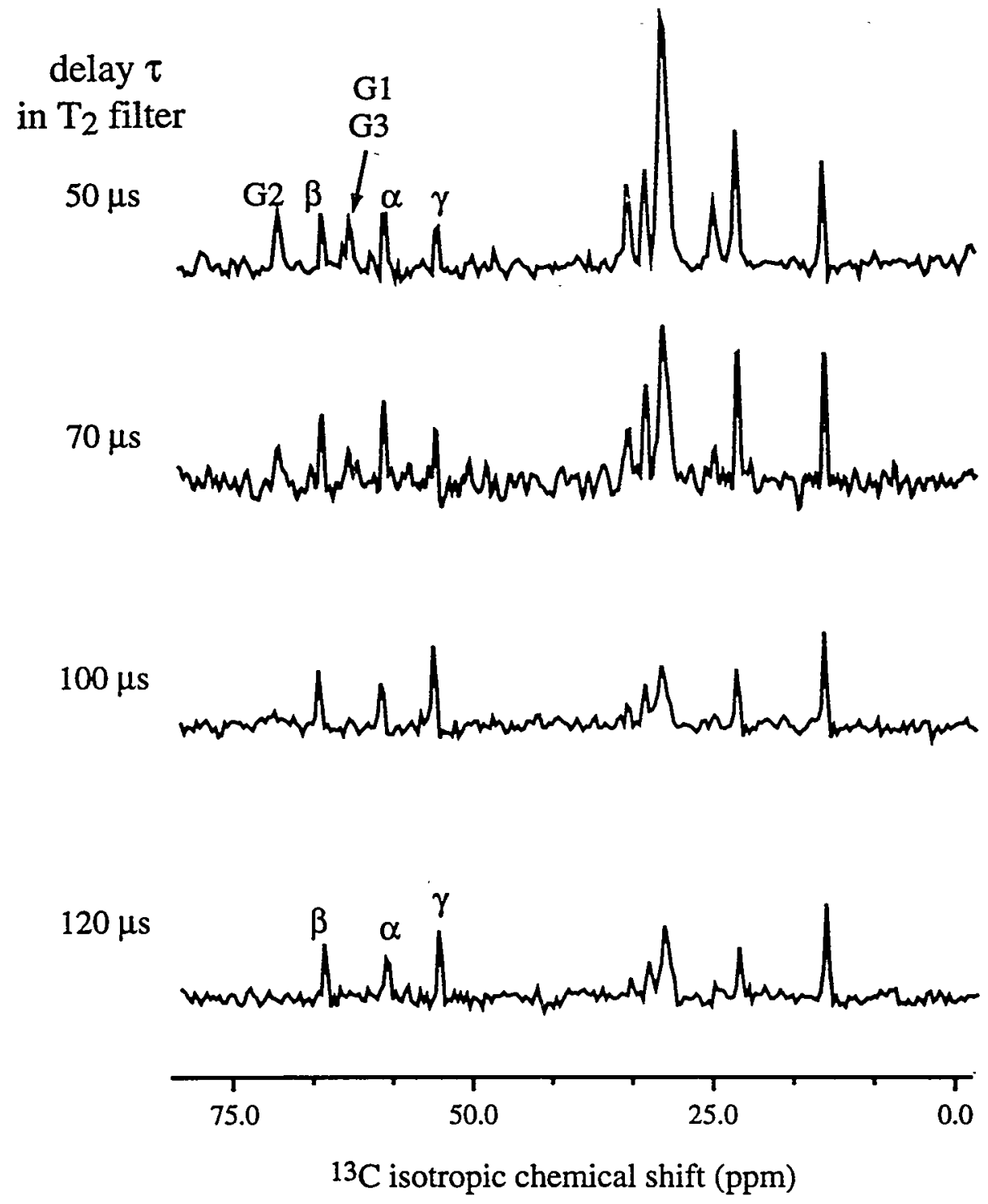

Figure 5.10 DMPC ${ }^{13} \mathrm{C}$ CPMAS spectra with ${ }^{1} \mathrm{H} \mathrm{T}_{2}$ filter of varying duration $\tau$. The glycerol signals are suppressed at $\tau>100 \mu \mathrm{s}$. CP contact time: $2 \mathrm{~ms}$. 
dipolar couplings of $120 \mathrm{~Hz}$ or less, the CP transfer curve is still within the initial rise after $2 \mathrm{~ms}$. It is also verified experimentally that this $2 \mathrm{~ms}$ contact time falls within the initial munotonic rise of the $\mathrm{CP}$ efficiencies for all the $\mathrm{CO} 2-\mathrm{H}$ dipolar pairs.

\section{$5.4{ }^{31} \mathrm{P}-1 \mathrm{H}$ Dipolar Couplings}

In addition to the segmental $\mathrm{C}-\mathrm{H}$ couplings, dipolar couplings between the ${ }^{31} \mathrm{P}$ and the protons in the headgrolip and glycerol regions of $L_{\alpha}$-phosphocholine can also be measured by the PDLF technique. The ${ }^{31} \mathrm{P}-{ }^{1} \mathrm{H}$ PDLF experiment employs the same pulse sequence as the one used for measuring the short-range $\mathrm{C}-\mathrm{H}$ couplings, except without sample rotation. Thus the $\mathrm{P}-\mathrm{H}$ couplings are correlated with the anisotropic ${ }^{31} \mathrm{P}$ chemical shift. Sample spinning is unnecessary because only one ${ }^{31} \mathrm{P}$ spin is present in the molecule and site resolution is $100 \%$. The inhomogeneous ${ }^{31} \mathrm{P}$ powder pattern enhances the $2 \mathrm{D}$ spectral resolution by removing orientational broadening in the dipolar coupling cross sections. Since all motionally-averaged NMR interactions in phospholipid bilayers are uniaxially parallel to the director, the correlation between the dipolar couplings and chemical shift anisotropies results in a 2D spectrum whose cross sections in either dimension are free of orientational broadening.

Figure 5.11a displays a P-H 2D PDLF spectrum of lecithin. The $\mathrm{P}-\mathrm{H}$ doublets in the dipolar dimension are symmetric with respect to $\omega_{1}=0$ due to the exclusive detection of the cosine-modulated dipolar coherence at the end of $t_{1}$. The $\omega_{2}$ dimension shows the ${ }^{31} \mathrm{P}$ chemical shift powder pattern after cross polarization from ${ }^{1} \mathrm{H}$, with a distinct "magic-angle hole" at the ${ }^{31} \mathrm{P}$ isotropic chemical shift. The values of the $\mathrm{P}-\mathrm{H}$ dipolar couplings can be extracted most accurately from the cross section at the maximum (i.e. the $90^{\circ}$ edge) of the ${ }^{31} \mathrm{P}$ powder pattern, which corresponds to those directors perpendicular to the magnetic field. The $\mathrm{P}-\mathrm{H}$ doublet in this slice has a splitting of $\sim 280 \mathrm{~Hz}$ (Figure $5.11 \mathrm{~b}$ ), and is the superposition of several $\mathrm{P}-\mathrm{H}$ couplings between ${ }^{31} \mathrm{P}$ and the protons in the headgroup and glycerol segments. 
The P-H 2D PDLF experiment is also applied to PE (305 K) and SPM $(320 \mathrm{~K})$ to probe the conformational similarity of the phosphate group among these molecules. The $\mathrm{PE}$ and SPM dipolar spectra, extracted at the maxima of the ${ }^{31} \mathrm{P}$ powder patterns, are shown in Figure 5.11(c-d). The SPM spectrum exhibits a splitting of about $210 \mathrm{~Hz}$, compared to $280 \mathrm{~Hz}$ for lecithin. Its relative intensity of the zero-frequency peak and the general lineshape differ markedly from those of the lecithin spectrum. This suggests that the headgroups of lecithin and SPM have quite different conformations, despite their similar chemical structures and similar ${ }^{31} \mathrm{P}$ chemical shift spectra. The P-H splitting in PE is about $230 \mathrm{~Hz}$, which is intermediate between that of lecithin and SPM. The PE spectrum exhibits no zero-frequency peak, indicating that the central frequency peaks in the lecithin and SPM spectra arise from weak unresolved $\mathrm{P}-\mathrm{H}$ couplings.

P-H couplings are long-range interactions that depend on internuclear distances, orientations of the internuclear vectors with respect to the director, and internal molecular motions. Specifically, all protons are at least three bonds away from the ${ }^{31} \mathrm{P}$ nucleus so that the P-H distances depend on one or several torsion angles. Therefore, the P-H dipolar couplings provide long-range constraints on the structures of the phospholipid headgroup and glycerol backbone, but cannot be used for characterizing the order tensor of a segment.

The individual $\mathrm{P}-\mathrm{H}$ dipolar couplings within the superposed $\mathrm{P}-\mathrm{H}$ doublets in the cross sections of Figure 5.11 can be separated experimentally by correlating the $\mathrm{P}-\mathrm{H}$ couplings with additional NMR interactions, namely the $\mathrm{H}-\mathrm{H}$ homonuclear couplings and ${ }^{1} \mathrm{H}$ chemical shifts. To avoid a 3D experiment involving, for example, $\mathrm{P}-\mathrm{H}$ couplings, ${ }^{31} \mathrm{P}$ chemical shift and $\mathrm{H}-\mathrm{H}$ couplings, we can remove the ${ }^{31} \mathrm{P}$ chemical shift dimension by spectroscopically selecting a specific orientation of bilayers. 
(a)

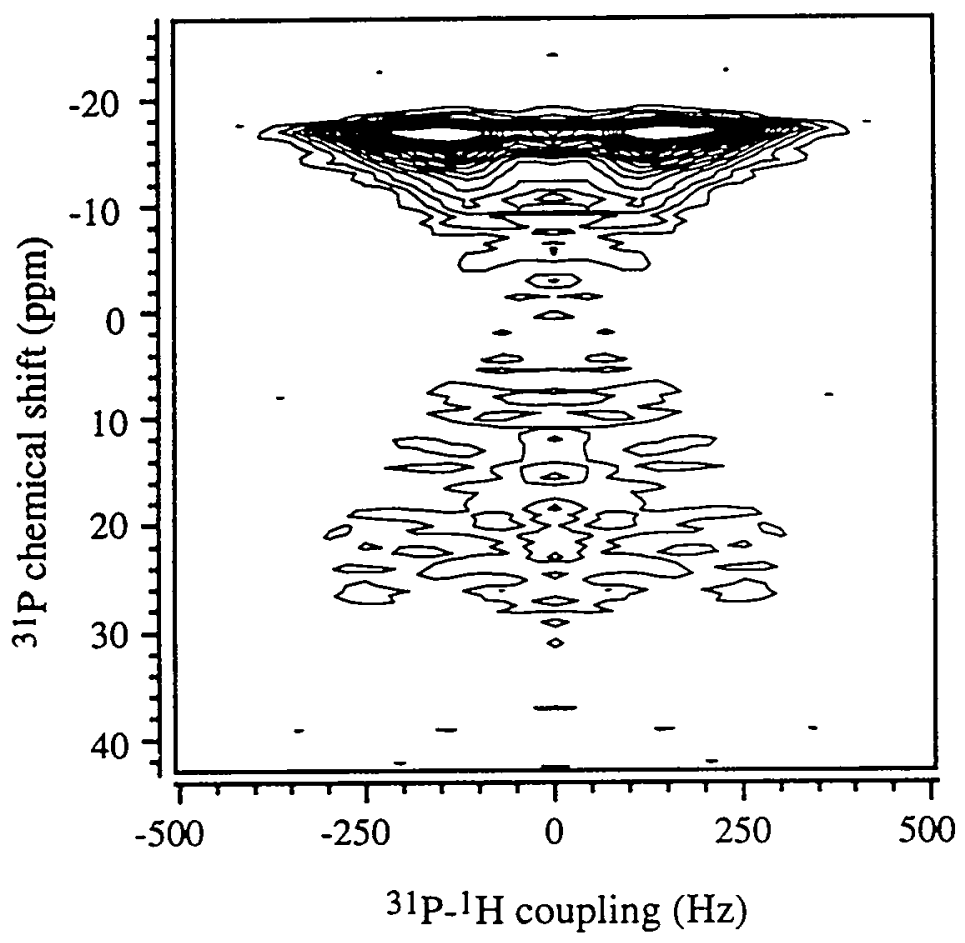

(b) $\mathrm{PC}: 280 \mathrm{~Hz}$

(c) $\mathrm{PE}: 230 \mathrm{~Hz}$

(d) $\mathrm{SPM}: 210 \mathrm{~Hz}$

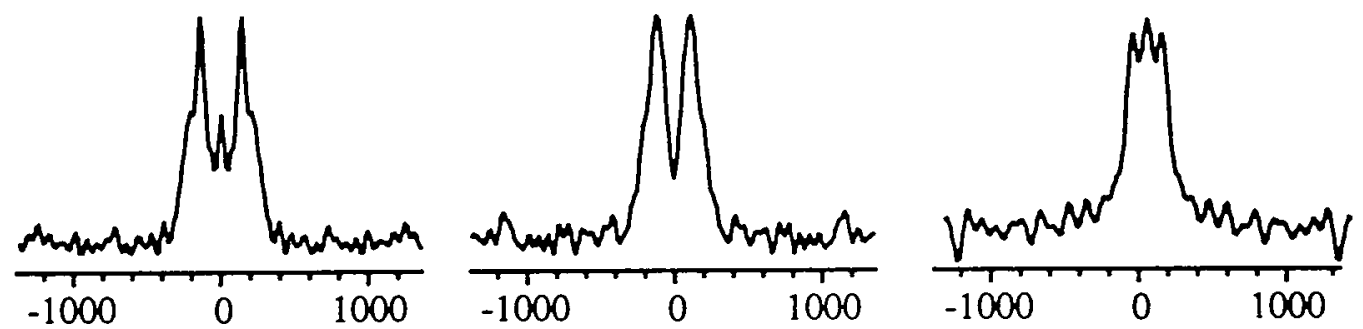

Figure 5.11 P-H/31 P CSA 2D correlation spectra of three $\mathrm{L}_{\alpha}$-phospholipids. (a) $2 \mathrm{D}$ spectrum of lecithin obtained under the static condition. P-H cross sections for (b) PC, (c) SPM, and (d) PE lipids are taken from the $90^{\circ}$ edge of the ${ }^{31} \mathrm{P}$ chemical shift powder patterns. 
The pulse sequence for correlating the $\mathrm{P}-\mathrm{H}$ and $\mathrm{H}-\mathrm{H}$ dipolar couplings is shown in Figure 5.12. A ${ }^{31} \mathrm{P} 90^{\circ}$ pulse excites the ${ }^{31} \mathrm{P}$ magnetization, which is transferred to ${ }^{1} \mathrm{H}$ by cross polarization. The ${ }^{1} \mathrm{H}$ magnetization evolves during the $t_{1}$ period under $\mathrm{P}-\mathrm{H}$ dipolar couplings. The $\mathrm{H}-\mathrm{H}$ couplings and ${ }^{1} \mathrm{H}$ chemical shifts are refocused respectively by a MREV-8 multiple-pulse sequence and a ${ }^{1} \mathrm{H} 180^{\circ}$ pulse in the middle of $t_{1}$. The ${ }^{1} \mathrm{H}$ magnetization is then detected in $t_{2}$-without further spin manipulation. Since the homonuclear coupling is by far the strongest interaction experienced by the protons, the $\omega_{2}$ dimension is dominated by the $\mathrm{H}-\mathrm{H}$ couplings.

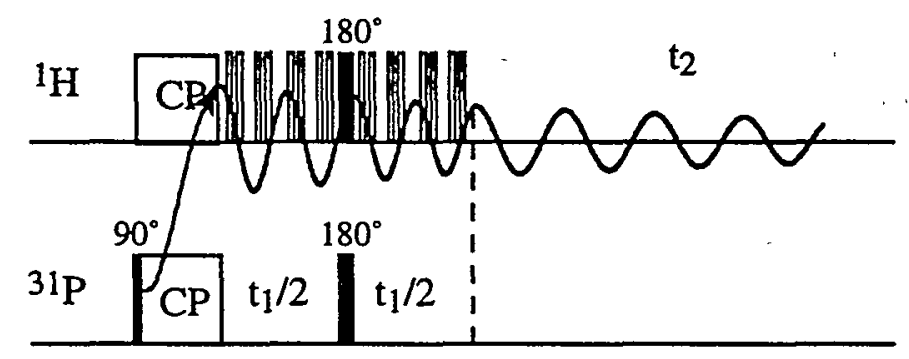

Figure 5.12 Pulse sequence correlating $\mathrm{P}-\mathrm{H}$ and $\mathrm{H}-\mathrm{H}$ couplings. All proton interactions are retained in $t_{2}$, but the strongest interaction is the $\mathrm{H}-\mathrm{H}$ homonuclear coupling.

Normally, broad ${ }^{1} \mathrm{H}$ lines would be expected in the $\omega_{2}$ dimension due to the ${ }^{1} \mathrm{H}$ multiple-spin interaction and the isotropic distribution of bilayer orientations. However, in the current experiment, orientational broadening is alleviated by the spectroscopic selection of a narrow range of bilayer orientations, namely the directors that are normal to the magnetic field. This is achieved by placing the ${ }^{31} \mathrm{P}$ resonance frequency at the $90^{\circ}$ edge of the ${ }^{31} \mathrm{P}$ powder pattern. In addition, the ${ }^{31} \mathrm{P}$ spin-lock field during $\mathrm{P} \rightarrow \mathrm{H} \mathrm{CP}$ is smaller than the ${ }^{31} \mathrm{P}$ chemical shift spectral width, so the excitation range is limited to the region around the ${ }^{31} \mathrm{P}$ powder maximum. Finally, the "magic-angle hole" at the ${ }^{31} \mathrm{P}$ isotropic chemical shift reinforces the orientation selection. 
(a)

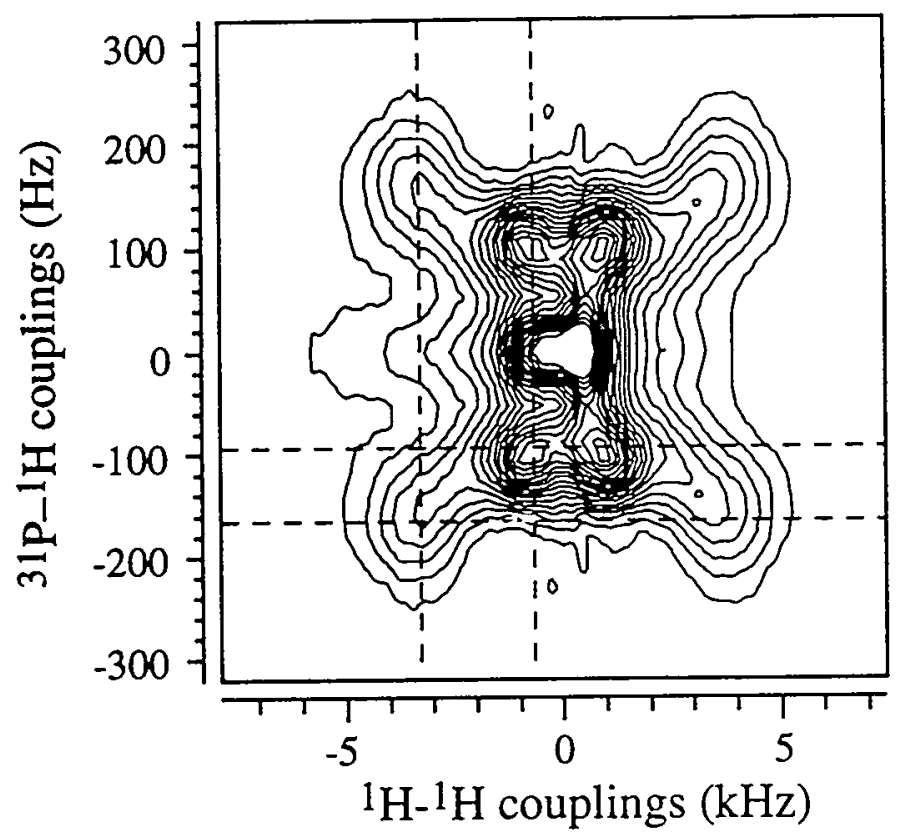

(b)

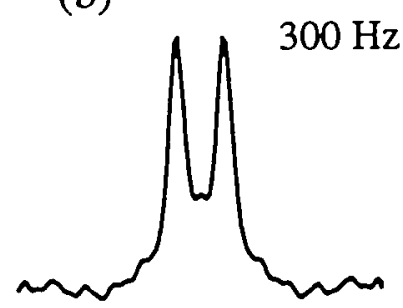

(c)
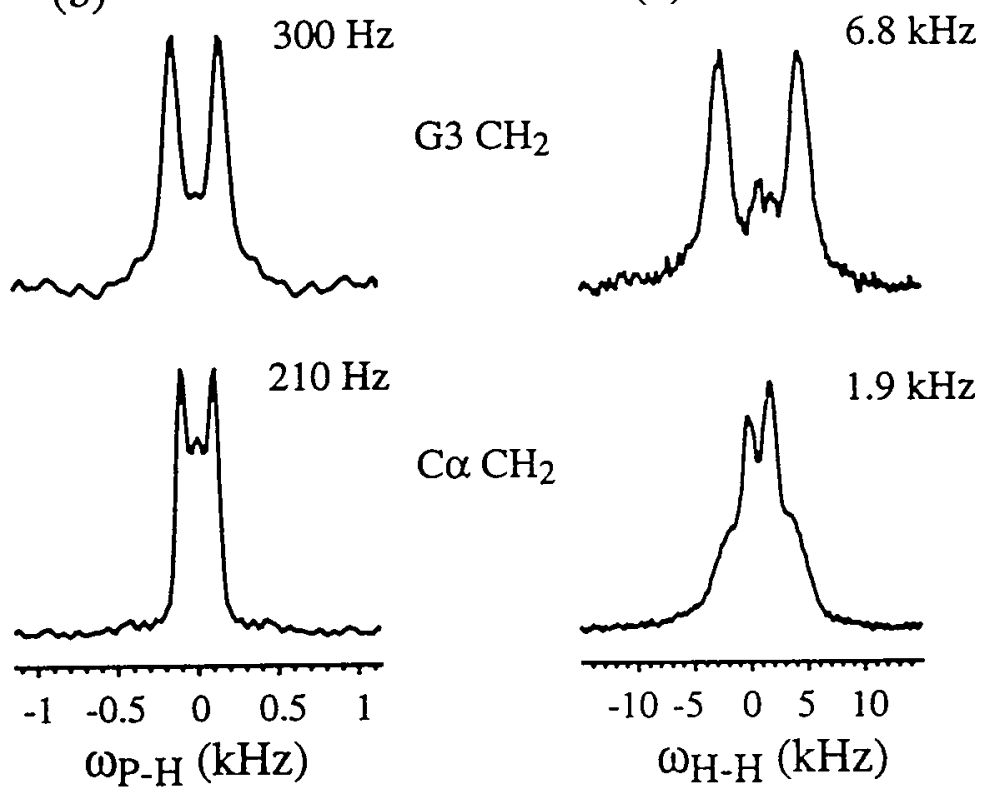

Figure 5.13 $\mathrm{L}_{\alpha}$-lecithin $\mathrm{P}-\mathrm{H} / \mathrm{H}-\mathrm{H}$ correlation spectrum. (a) 2D spectrum. (b) Two $\mathrm{P}-\mathrm{H}$ cross sections. (c) The corresponding $\mathrm{H}-\mathrm{H}$ cross sections. 
The $\mathrm{P}-\mathrm{H} / \mathrm{H}-\mathrm{H}$ correlation spectrum of lecithin is shown in Figure 5.13a. Two major $\mathrm{P}-\mathrm{H}$ doublets are resolved, with the larger $\mathrm{P}-\mathrm{H}$ splitting correlated with the larger $\mathrm{H}-\mathrm{H}$ splitting in the other dimension. The magnitudes of the $\mathrm{P}-\mathrm{H}$ couplings are $300 \pm 30$ $\mathrm{Hz}$ and $210 \pm 20 \mathrm{~Hz}$, as shown in the two $\mathrm{P}-\mathrm{H}$ cross sections (Figure 5.13b). These couplings can be tentatively assigned to the glycerol $\mathrm{H}_{\mathrm{G} 3}$ protons and the headgroup $\mathrm{H} \alpha$ protons since they are closest to the phosphate unit. This assignment can be corroborated by $\mathrm{H}-\mathrm{H}$ couplings as described below.

\section{$5.5{ }^{1} \mathrm{H}-{ }^{1} \mathrm{H}$ Dipolar Couplings}

Geminal ${ }^{1} \mathrm{H}-{ }^{1} \mathrm{H}$ couplings in the headgroup and glycerol segments provide important information on the order tensors of these segments, because the $\mathrm{H}-\mathrm{H}$ vector is parallel to one of the three principal axes of the order tensor. This colinearity results from the degeneracy of the $\mathrm{C}-\mathrm{H}$ bond order parameters in each methylene group, as shown by ${ }^{2} \mathrm{H}$ quadrupolar splittings and $\mathrm{C}-\mathrm{H}$ dipolar couplings.

One way of obtaining geminal $\mathrm{H}-\mathrm{H}$ couplings is to correlate them with $\mathrm{P}-\mathrm{H}$ couplings using the pulse sequence of Figure 5.12. In the resulting $\mathrm{P}-\mathrm{H} / \mathrm{H}-\mathrm{H} 2 \mathrm{D}$ correlation spectrum, two $\mathrm{H}-\mathrm{H}$ doublets with splittings of $6.8 \pm 0.4 \mathrm{kHz}$ and $1.9 \pm 0.4 \mathrm{kHz}$ are observed, as shown in Figure 5.13c. The remarkable resolution of these $\mathrm{H}-\mathrm{H}$ couplings can be attributed to the orientation selection technique applied and the inhomogeneity of the ${ }^{1} \mathrm{H}$ spin system ${ }^{19}$ in the liquid-crystalline phosphocholine. Considerations of $\mathrm{P}-\mathrm{H}$ distances suggest that these two $\mathrm{H}-\mathrm{H}$ couplings may be assigned to the glycerol $\mathrm{H}_{\mathrm{G} 3}$ and the headgroup $\mathrm{H} \alpha$ protons. This assignment can be confirmed by correlating the $\mathrm{H}-\mathrm{H}$ couplings with ${ }^{1} \mathrm{H}$ chemical shifts in a modified version of the last experiment. By removing the ${ }^{1} \mathrm{H} 180^{\circ}$ pulse in the $t_{1}$ period, the ${ }^{1} \mathrm{H}$ chemical shift frequencies are recorded while the $\mathrm{P}-\mathrm{H}$ dipolar couplings are refocused.

Two $\mathrm{H}-\mathrm{H}$ cross sections from a ${ }^{1} \mathrm{H}$ dipolar-shift correlation spectrum are shown in Figure 5.14. In the cross section at $4.2 \mathrm{ppm}$ (Figure 5.14a), two H-H splittings of 
$6.2 \pm 0.7 \mathrm{kHz}$ and $1.8 \pm 0.4 \mathrm{kHz}$ can be resolved. To determine which sites this chemical shift correspond to, we need to examine the ${ }^{1} \mathrm{H}$ chemical shift more closely. The isotropic ${ }^{1} \mathrm{H}$ chemical shifts of various segments in lecithin can be determined precisely by a ${ }^{13} \mathrm{C} /{ }^{1} \mathrm{H}$ MAS HETCOR experiment.

(a) $4.2 \mathrm{ppm}$

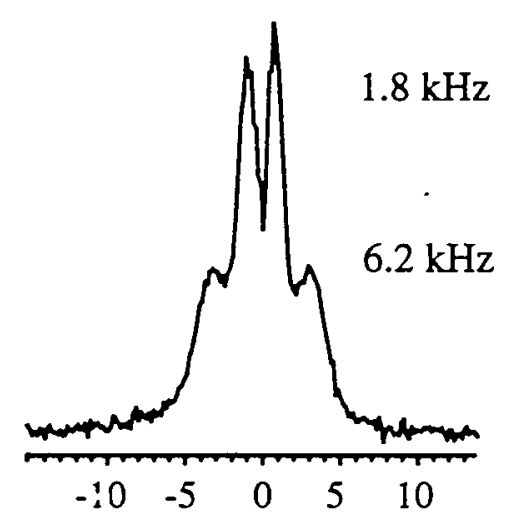

(b) $5.3 \mathrm{ppm}$

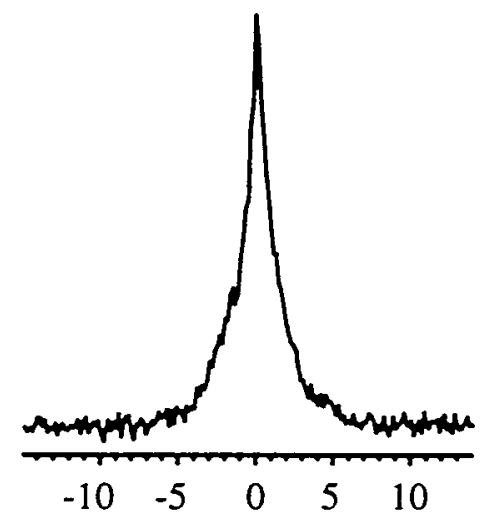

1 $\mathrm{H}-1 \mathrm{H}$ dipolar coupling $(\mathrm{kHz})$

Figure 5.14 $\mathrm{L}_{\alpha}$-lecithin $\mathrm{H}-\mathrm{H} /{ }^{1} \mathrm{H}$ CSA correlation spectrum. (a) $\mathrm{H} \alpha$ and $\mathrm{H}_{\mathrm{G} 3}$ slices at ${ }^{1} \mathrm{H}$ chemical shift of $4.2 \mathrm{ppm}$. (b) $\mathrm{H}_{\mathrm{G} 2}$ slice at ${ }^{1} \mathrm{H}$ chemical shift of $5.3 \mathrm{ppm}$.

A $2 \mathrm{D}{ }^{13 / 1} \mathrm{H}$ MAS HETCOR spectrum is displayed in Figure 5.15. The $\mathrm{H}_{\mathrm{G} 2}$ proton resonates at $5.3 \mathrm{ppm}$ while the $\mathrm{H} \beta$ protons resonate at $3.8 \mathrm{ppm}$. These are well separated from the $\mathrm{H}_{\mathrm{G} 3}$ and $\mathrm{H} \alpha$ signals at $4.2 \mathrm{ppm}$. On the other hand, the anisotropic ${ }^{1} \mathrm{H}$ chemical shifts are expected to be motionally narrowed from a full width of about $6 \mathrm{ppm}$ to about 1.5 ppm (for glycerol protons) or less ( $0.4 \mathrm{ppm}$ for headgroup protons). From this information, we can assign the signals at $4.2 \mathrm{ppm}$ (Figure 5.14a) to the $\mathrm{H}_{\mathrm{G} 3}$ and $\mathrm{H} \alpha$ protons. This confirms our assignment of the $\mathrm{H}-\mathrm{H}$ splittings $(6.5 \mathrm{kHz}$ and $1.8 \mathrm{kHz})$ to the $\mathrm{H}_{\mathrm{G} 3}$ and $\mathrm{H} \alpha$ protons in the $\mathrm{P}-\mathrm{H} / \mathrm{H}-\mathrm{H}$ spectrum.

Figure $5.14 \mathrm{~b}$ shows the $\mathrm{H}-\mathrm{H}$ coupling cross section at $5.3 \mathrm{ppm}$, corresponding to the chemical shift of the glycerol $\mathrm{H}_{\mathrm{G} 2}$ proton. It exhibits a hump without a resolved splitting, which is consistent with the presence of only one proton in this segment. 


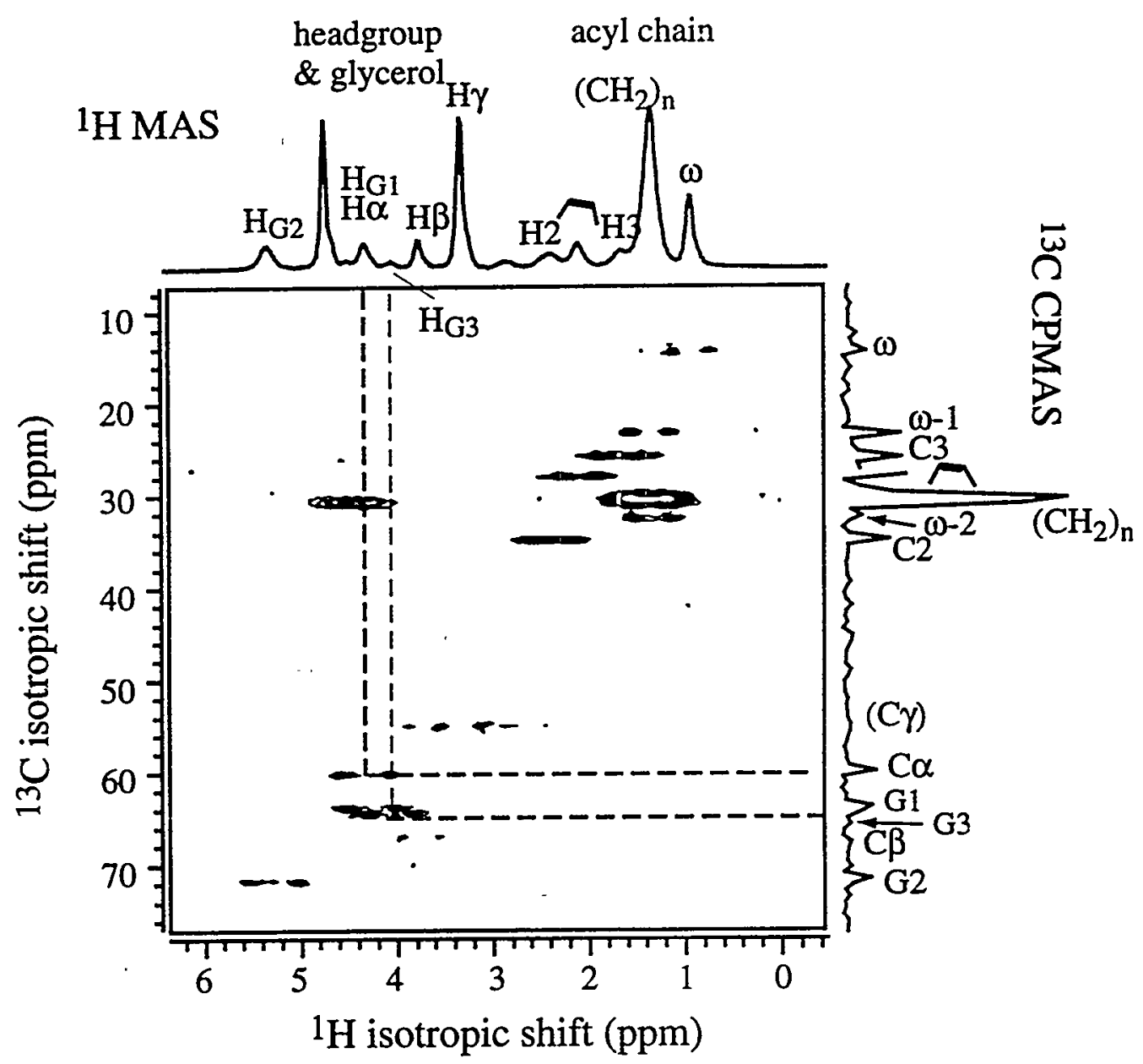

Figure 5.15 $\mathrm{L}_{\alpha}$-lecithin ${ }^{13} \mathrm{C} /{ }^{1} \mathrm{H}$ MAS chemical shift correlation spectrum. 1D MAS spectra are shown along the two dimensions for comparison. The sample was spun at 2 kHz. 
So far, we have not determined which $\mathrm{H}-\mathrm{H}$ dipolar coupling results from the $\mathrm{H}_{\mathrm{G} 3}$

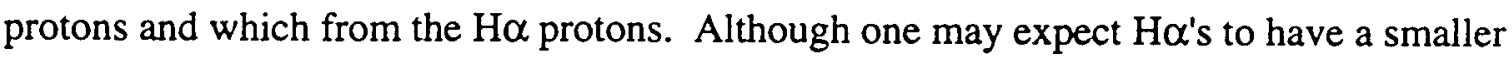
$\mathrm{H}-\mathrm{H}$ splitting since the headgroup is more mobile than the glycerol backbone, this hypothesis needs to be tested experimentally. A related problem is that, due to the static condition for the ${ }^{1} \mathrm{H}$ dipolar-shift experiment, the chemical shifi dimension has low site resolution. Better -resolved ${ }^{1} \mathrm{H}$ chemical shift spectra can be obtained under switchingangle spinning, by correlating isotropic ${ }^{1} \mathrm{H}$ chemical shifts with anisotropic $\mathrm{H}-\mathrm{H}$ homonuclear couplings, analogous to the Wideline Separation (WISE) technique. ${ }^{21}$ The WISE pulse sequence in Figure 5.16 shows that homonuclear $\mathrm{H}-\mathrm{H}$ dipolar couplings are measured in $t_{1}$ and are correlated with the isotropic ${ }^{1} \mathrm{H}$ chemical shifts detected in $t_{2}$. In the resulting spectrum, the ${ }^{1} \mathrm{H}$ MAS dimension should exhibit spinning sidebands and a centerband consisting of well-resolved ${ }^{1} \mathrm{H}$ isotropic peaks. In the other dimension, the ${ }^{1} \mathrm{H}$ spectral widths provide semiquantitative values of the $\mathrm{H}-\mathrm{H}$ homonuclear couplings.

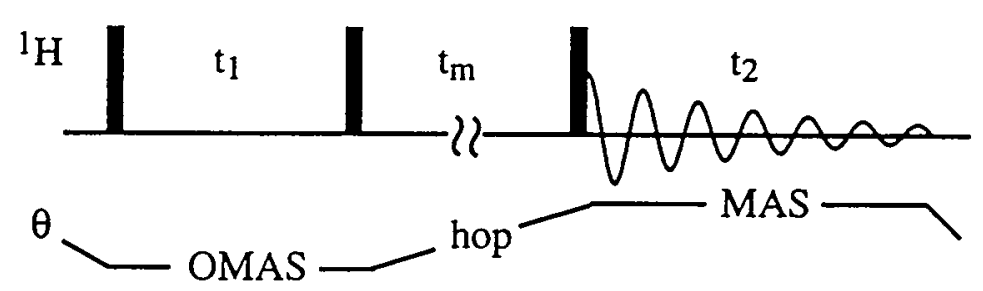

Figure $5.16{ }^{1} \mathrm{H}$ WISE pulse sequence under SAS. $\mathrm{H}-\mathrm{H}$ couplings are separated according to ${ }^{1} \mathrm{H}$ isotropic chemical shifts.

The ${ }^{1} \mathrm{H}$ WISE spectrum of lecithin taken with an OMAS scaling factor of -0.3 is shown in Figure 5.17. The $\mathrm{H} \alpha$ slice, which is $0.3 \mathrm{ppm}$ from $\mathrm{H}_{\mathrm{G} 3}$ and closer to the water peak, shows a dipolar linewidth of $2.1 \mathrm{kHz}$. In comparison, the dipolar signals of the $\mathrm{H}_{\mathrm{G} 3}$ protons are too broad to be seen and show fast decays in the $t_{1}$ time domain. Therefore, the larger $\mathrm{H}-\mathrm{H}$ splitting in Figures 5.13 and 5.14 is assigned to $\mathrm{H}_{\mathrm{G} 3}$ protons, and the smaller coupling to the $H \alpha$ protons. 
An alternative WISE experiment involves cross polarization from ${ }^{1} \mathrm{H}$ to ${ }^{13} \mathrm{C}$ after the evolution period, so that the $\mathrm{H}-\mathrm{H}$ couplings can be correlated with well-resolved isotropic ${ }^{13} \mathrm{C}$ chemical shifts. Figure 5.18 shows a stack plot of the ${ }^{13} \mathrm{C}$-WISE spectrum of DMPC. Again, the $\mathrm{C} \alpha$ and $\mathrm{C} \beta$ slices exhibit smaller $\mathrm{H}-\mathrm{H}$ couplings $(\sim 1.6 \mathrm{kHz})$ than all the glycerol sites, whose ${ }^{1} \mathrm{H}$ spectra are too broad to be observed.

The reason that the WISE technique produces broad ${ }^{1} \mathrm{H}$ lines instead of resolved $\mathrm{H}-\mathrm{H}$ doublets is that no specific bilayer orientation is selected. The pulse sequence for the ${ }^{1} \mathrm{H}$ WISE experiment employs single pulse excitation instead of $\mathrm{P} \rightarrow \mathrm{H}$ cross polarization, as orientation selection by a selective ${ }^{31} \mathrm{P}$ pulse is not possible under sample spinning. Thus the homonuclear coupling patterns in the 2D WISE spectra are orientationally broadened. On the other hand, the presence of relatively well-resolved $\mathrm{H}-\mathrm{H}$ doublets in Figures 5.13 and 5.14 not only results from the orientation selection. These resolved doublets indicate an upper limit of $1 \mathrm{kHz}$ to the non-geminal $\mathrm{H}-\mathrm{H}$ couplings. This rather small value is consistent with the conclusions drawn from ${ }^{2} \mathrm{H}$ NMR. Namely, the fast exchange between mirror-symmetric headgroup conformations reduces the couplings between protons in different segments, while the couplings between geminal protons are invariant to the exchange. Therefore, in the headgroup and glycerol regions, the geminal $\mathrm{H}-\mathrm{H}$ couplings are much stronger than the non-geminal ones, and a simple doublet splitting results for each segment.

The lineshape of ${ }^{1} \mathrm{H}$ dipolar spectra of molecules undergoing uniaxial motions has been simulated previously. ${ }^{22}$ There, the Hamiltonian was taken to consist of a geminal H$\mathrm{H}$ coupling which produces a dipolar doublet, and lumped non-geminal $\mathrm{H}-\mathrm{H}$ couplings that produce Gaussian broadening. Both types of couplings scale as $P_{2}(\cos \theta)$, where $\theta$ is the angle between the director and the $B_{0}$ field. For very small non-geminal $\mathrm{H}-\mathrm{H}$ couplings, a Pake pattern is obtained. It was found that the larger the size of the broadening function relative to the geminal $\mathrm{H}-\mathrm{H}$ splitting, the more the spectral lineshape becomes logarithmic (or super-Lorentzian). Such a logarithmic lineshape exhibits a sharp 


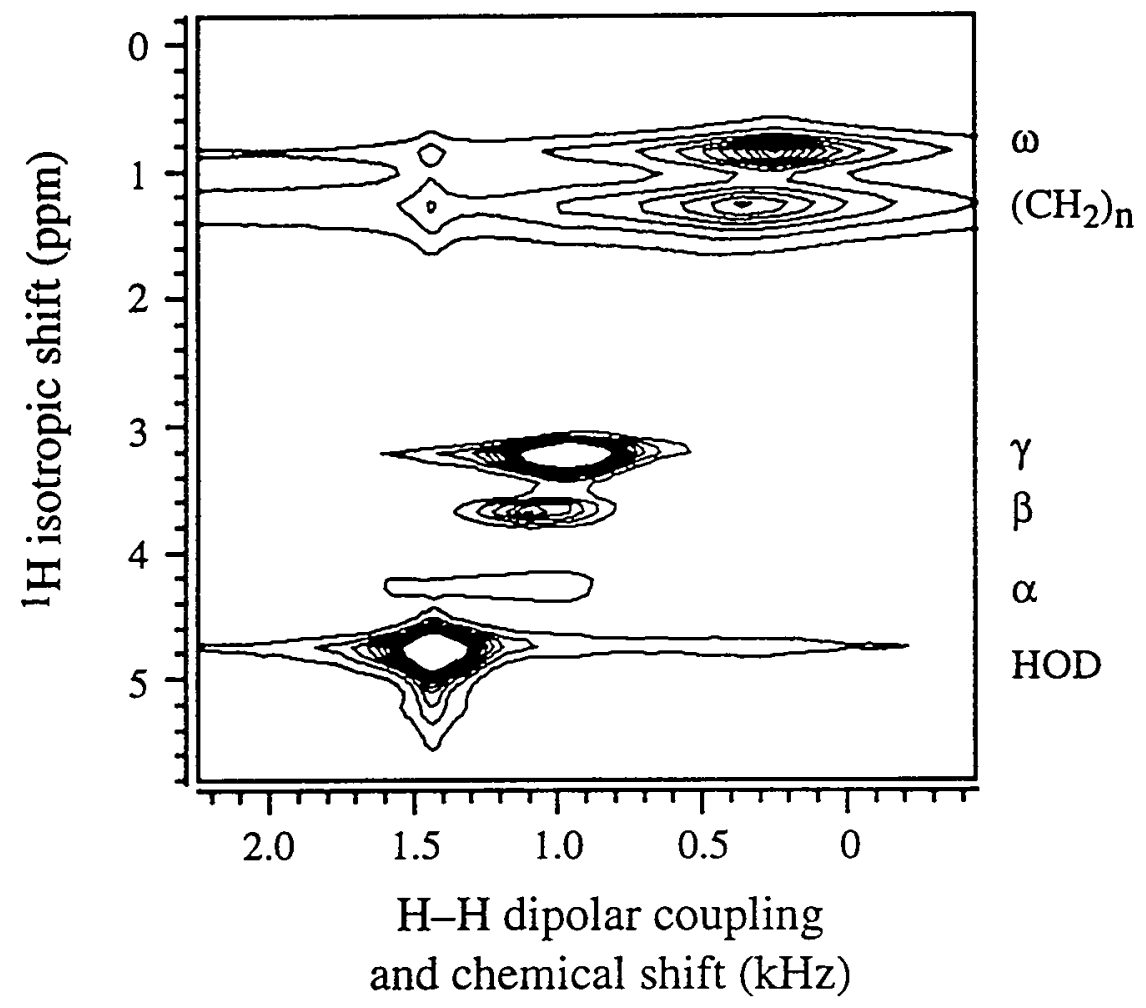

Figure 5.17 $\mathrm{L}_{\alpha}$-lecithin ${ }^{1} \mathrm{H}$ WISE spectrum obtained under SAS. Dipolar scaling factor: $P_{2}\left(\cos 69^{\circ}\right)=-0.3$. The sample was spun at $2 \mathrm{kHz}$. 


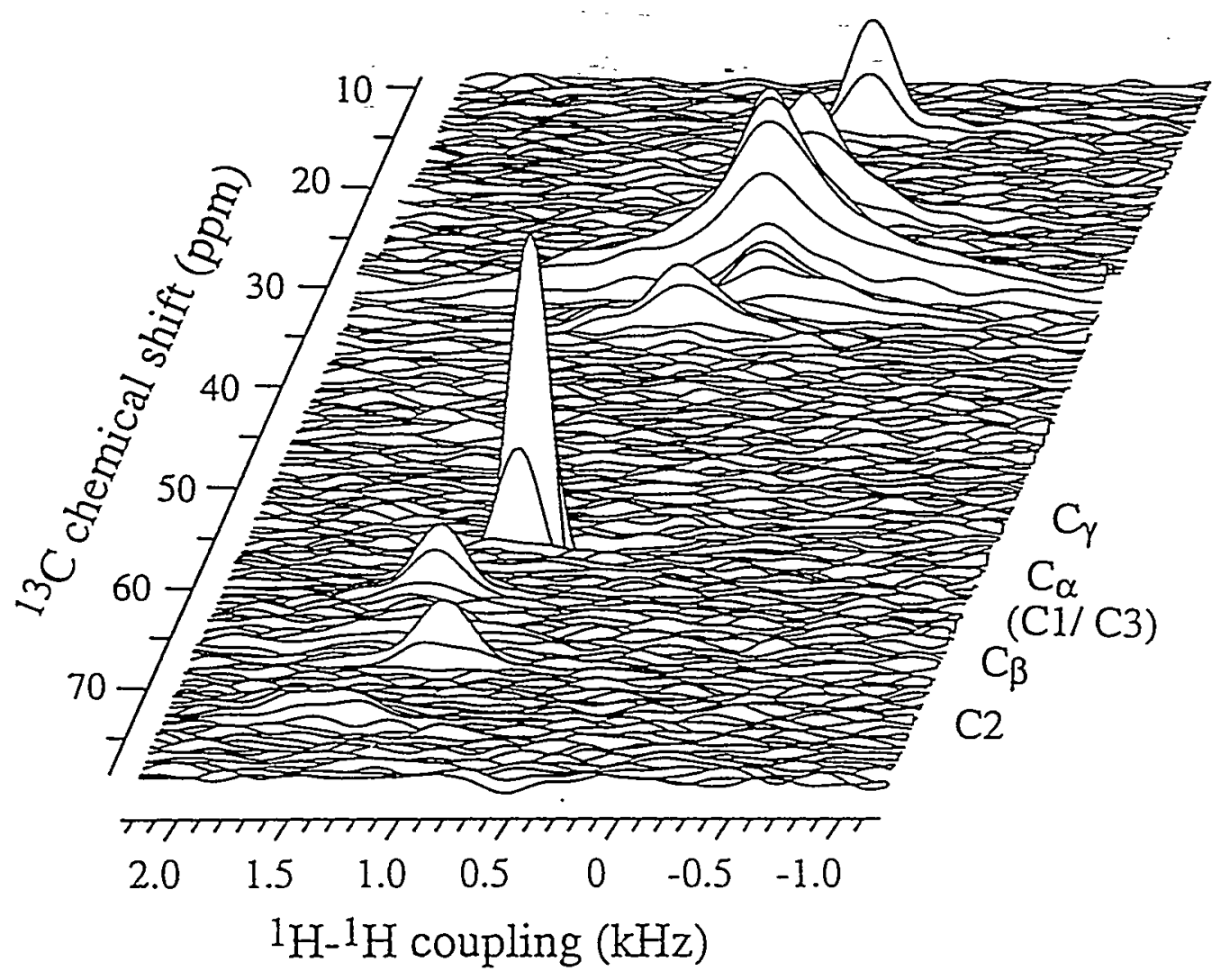

Figure 5.18 $\mathrm{L}_{\alpha}$-DMPC ${ }^{13} \mathrm{C}$ WISE spectrum obtained under OMAS. Dipolar scaling factor: $P_{2}\left(\cos 47^{\circ}\right)=+0.2$. The sample was spun at $2 \mathrm{kHz}$. 
cusp at the center, which corresponds to molecules oriented at the magic angle with respect to the $B_{0}$ field. Therefore, the half-width of this super-Lorentzian line has little meaning, since it strongly depends on the ratio of the geminal and non-geminal $\mathrm{H}-\mathrm{H}$ couplings as well as on the intrinsic width of the signal from the molecules subtending the magic angle. In the case of our ${ }^{1} \mathrm{H}$ WISE spectra, however, only the relative widths of the lines need to be considered, since we only need a qualitative assignment of the geminal $\mathrm{H}-\mathrm{H}$ splittings in the WISE spectra.

\section{$5.6{ }^{31} \mathrm{P}-{ }^{13} \mathrm{C}$ Dipolar Couplings}

Similar to the ${ }^{31} \mathrm{P}-1 \mathrm{H}$ couplings, ${ }^{31} \mathrm{P}-{ }^{13} \mathrm{C}$ dipolar couplings provide another type of long-range constraints on the conformation of the phospholipid headgroup and glycerol backbone. These couplings have been reported ${ }^{23,24}$ on DMPC oriented in detergent matrices, however extrapolation was necessary to obtain the $\mathrm{P}-\mathrm{C}$ couplings for the pure DMPC.

We measured the $\mathrm{P}-\mathrm{C}$ dipolar couplings in pure and unoriented lecithin by a simple ID OMAS experiment. Due to the parallelism of the NMR interactions in the $\mathrm{L}_{\alpha}$ phase, the ${ }^{13} \mathrm{C}$ chemical shift powder patterns should exhibit splittings due to the $\mathrm{P}-\mathrm{C}$ dipolar couplings. Indeed, in a ${ }^{13} \mathrm{C}$ spectrum with an OMAS scaling factor of $P_{2}\left(\cos 43^{\circ}\right)=+0.3$ (Figure 5.19a), the maxima of the chemical shift powder patterns of $\mathrm{C} \alpha$ and G2 are split by their couplings to ${ }^{31} \mathrm{P}$. The sizes of the splittings correspond to the $P_{2}$-scaled dipolar couplings. Comparison with a ${ }^{13} \mathrm{C}$ MAS spectrum (Figure $5.19 \mathrm{~b}$ ) indicates that the $P_{2}$ scaled ${ }^{13} \mathrm{C}$ chemical shift anisotropies range from $-5 \mathrm{ppm}(\mathrm{Gl})$ to $+3 \mathrm{ppm}(\mathrm{C} \alpha)$ for the headgroup and glycerol segments, and the full coupling strengths of $\mathrm{G} 2$ and $\mathrm{C} \alpha$ to ${ }^{31} \mathrm{P}$ are $155 \pm 15 \mathrm{~Hz}$.

Since the G3 carbon is closer to $31 \mathrm{P}$ than G2, a P-G3 splitting is expected. However, the G3 chemical shift powder pattern overlaps with those of $C \beta$ and $G 1$, so the $\mathrm{P}-\mathrm{G} 3$ splitting cannot be resolved in the spectrum. To resolve the $\mathrm{P}-\mathrm{C}$ dipolar couplings 
(a)

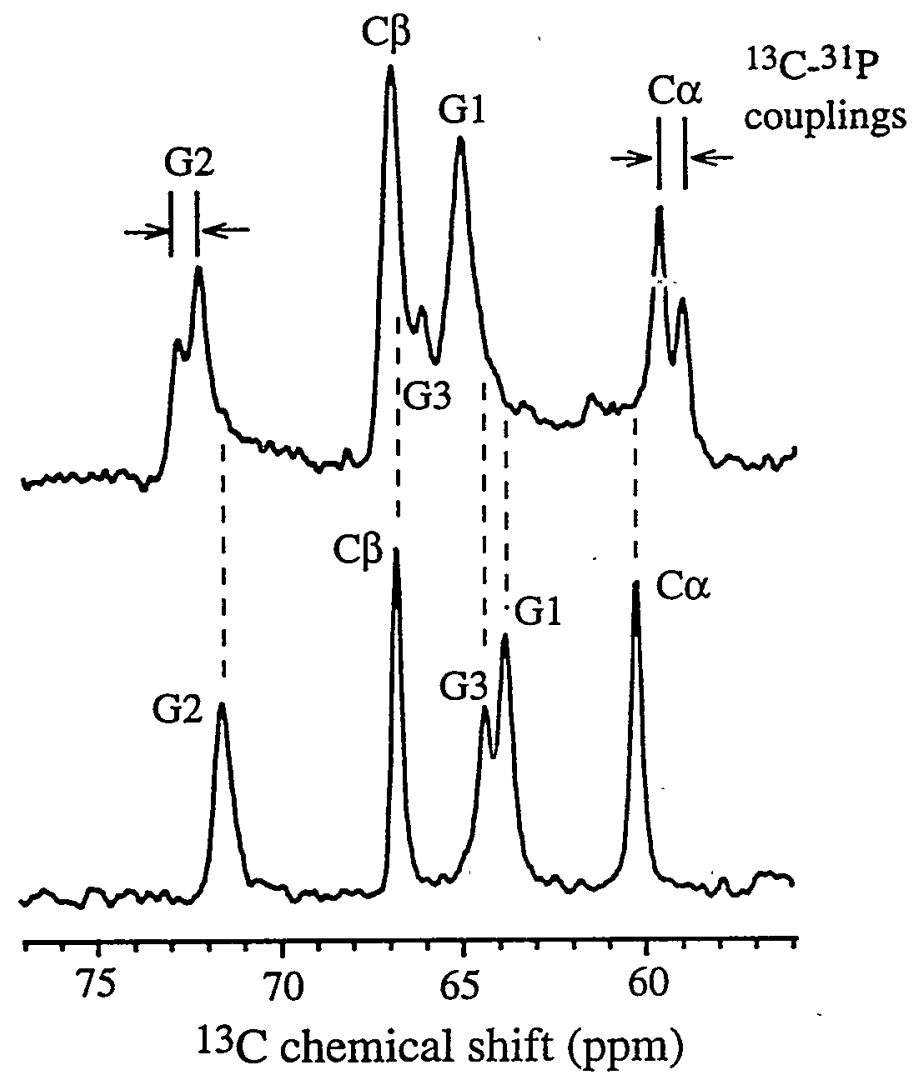

(c)

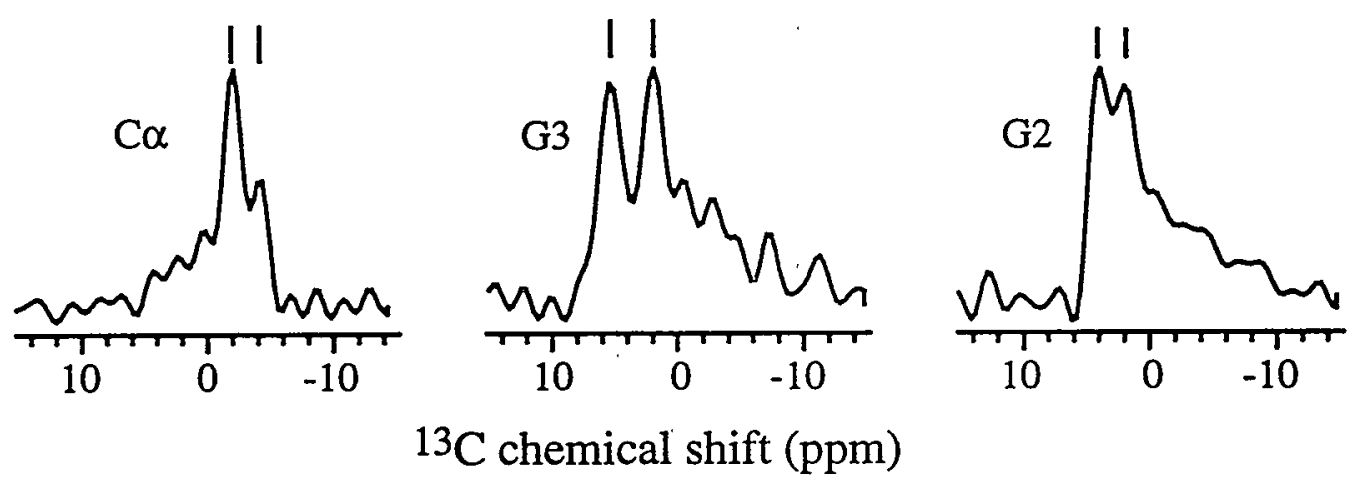

Figure 5.19 $\mathrm{L}_{\alpha}$-lecithin $\mathrm{P}-\mathrm{C}$ dipolar couplings. (a) ${ }^{13} \mathrm{C}$ OMAS spectrum in the headgroup and glycerol regions, scaled by $P_{2}\left(\cos 43^{\circ}\right)=+0.3$. (b) The corresponding ${ }^{13} \mathrm{C}$ MAS spectrum. (c) ${ }^{13} \mathrm{C}$ SAS cross sections of the $\mathrm{C} \alpha, \mathrm{G} 3$ and $\mathrm{G} 2$ sites. 
further, we carried out a 2D SAS experiment to separate the ${ }^{13} \mathrm{C} C S A$ s by the isotropic ${ }^{13} \mathrm{C}$ chemical shifts. Figure 5.19c displays the $\mathrm{C} \alpha, \mathrm{G} 3$ and $\mathrm{G} 2$ cross sections in a 2D SAS spectrum of lecithin. The $\mathrm{C} \alpha$ and $\mathrm{G} 2$ cross sections exhibit $\mathrm{P}-\mathrm{C}$ splittings of $168 \mathrm{~Hz}$ and $144 \mathrm{~Hz}$, respectively, whereas the G3 slice has the largest dipolar splitting of $264 \mathrm{~Hz}$. These P-C dipolar couplings are consistent with the results of the 1D OMAS spectrum within experimental error margins.

\section{$5.7{ }^{13} \mathrm{C}-{ }^{13} \mathrm{C}$ Dipolar Couplings}

As shown in section 5.3, long-range $\mathrm{C}-\mathrm{H}$ dipolar couplings involving the $\mathrm{CO} 2$ carbon are sensitive probes of the conformation of the DMPC molecule in the headgroup and the upper acyl chain regions. We can exploit the same labeled carbon to characterize the segmental orientation in the vicinity of $\mathrm{CO} 2$. For example, the motionally-averaged $\mathrm{CO} 2-\mathrm{C} 2$ dipolar coupling can be measured to give the order parameter of the $\mathrm{CO} 2-\mathrm{C} 2$ bond. Interference from dipolar couplings between $\mathrm{C} 2$ and carbons other than $\mathrm{CO} 2$ is negligible, since the low natural abundance of ${ }^{13} \mathrm{C}$ dictates that the probability of having a third ${ }^{13} \mathrm{C}$ spin near $\mathrm{C} 2$ is only $1.1 \%$.

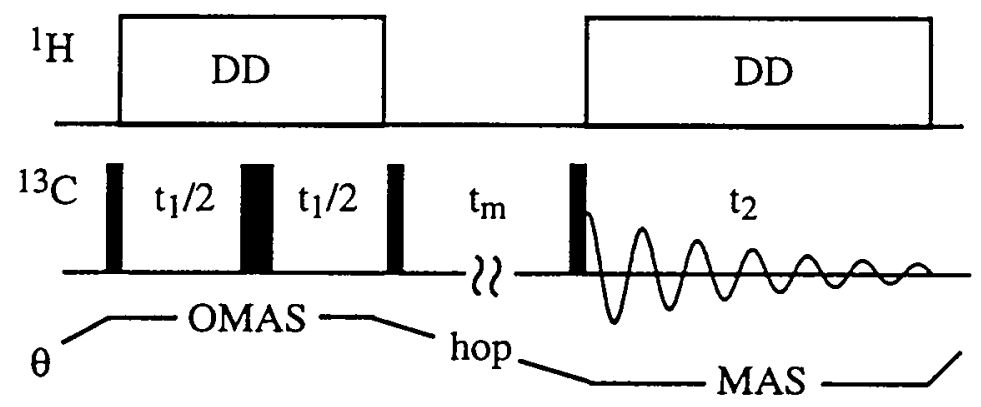

Figure 5.20 ${ }^{13} \mathrm{C}$ SLF pulse sequence under SAS.

The measurement of the $\mathrm{C}-\mathrm{C}$ dipolar coupling between the $\mathrm{CO} 2$ and the $\mathrm{C} 2$ carbon is achieved by separated-local-field spectroscopy ${ }^{25}$ under SAS (Figure 5.20). ${ }^{26,27}$ Following direct excitation of the ${ }^{13} \mathrm{C}$ spins, ${ }^{13} \mathrm{C}$ magnetization evolves under $\mathrm{C}-\mathrm{C}$ dipolar 
and scalar couplings. $\mathrm{A}^{13} \mathrm{C} 180^{\circ}$ pulse in the middle of $t_{1}$ refocuses the ${ }^{13} \mathrm{C}$ chemical shift while leaving the $\mathrm{C}-\mathrm{C}$ dipole interaction unaffected. The $\mathrm{C}-\mathrm{H}$ couplings are removed by continuous irradiation on ${ }^{1} \mathrm{H}$. The sample is spun off the magic angle during the $t_{1}$ period, and hopped to the magic angle in the mixing period $t_{m}$. During the $t_{2}$ period, the ${ }^{13} \mathrm{C}$ isotropic chemical shift is detected.

Figure 5.21 displays the contour plot of a SLF spectrum of DMPC, between 32 ppm and $36.5 \mathrm{ppm}$ of ${ }^{13} \mathrm{C}$ isotropic chemical shifts. The spinning angle during $t_{1}$ is $69^{\circ}$, so the $\mathrm{C}-\mathrm{C}$ dipole couplings are scaled by $P_{2}\left(\cos 69^{\circ}\right)=-0.3$. Most carbon sites exhibit only an unresolved peak at the zero frequency of the dipolar dimension, which reflects the lack of significant $\mathrm{C}-\mathrm{C}$ dipolar couplings to the $\mathrm{CO} 2$ carbon. However, in the $\mathrm{C} 2$ chemical shift region, a "butterfly" pattern is observed. It is the product of two splittings, generated by the $\mathrm{C}-\mathrm{C}$ scalar coupling in the isotropic dimension and the sum of dipolar and scalar couplings in the indirectly-detected, anisotropic dimension. The splitting in the isotropic dimension is about $55 \mathrm{~Hz}$, which agrees with the literature value of $52 \mathrm{~Hz}$ for ${ }^{1} J_{C C} .^{28}$ Extracting two cross sections along the indirect dimension at the two ends of this "butterfly", we find doublets with identical splittings of $47 \mathrm{~Hz}$ (Figure 5.21b). The dipolar contribution to the splitting is the motionally-averaged coupling constant $\bar{\delta}_{C C}$ scaled by the spinning factor -0.3 .

To obtain the sign and magnitude of the $\mathrm{CO} 2-\mathrm{C} 2$ dipolar coupling, we carried out another SLF experiment at a spinning angle of $43^{\circ}$, which corresponds to $P_{2}=+0.3$. For each site, if one of the splittings in the two spectra is smaller than ${ }^{1} J_{C C}$, then the average of the two splittings is the $J$ coupling, and the difference corresponds to twice the magnitude of the effective dipolar coupling constant, $\left|P_{2} \cdot \bar{\delta}_{C C}\right|:$ Moreover, if the splitting for the negative $P_{2}$ scaling factor is smaller than for the positive one, then $\bar{\delta}_{C C}$ is negative (see Figure 5.3). The SLF spectrum taken at $43^{\circ}$ is shown in Figure 5.22a. Again, a butterfly pattern is observed at the $\mathrm{C} 2$ chemical shift, and the doublet splittings in the two cross sections are found to be $70 \mathrm{~Hz}$ (Figure $5.22 \mathrm{~b}$ ). 
(a)
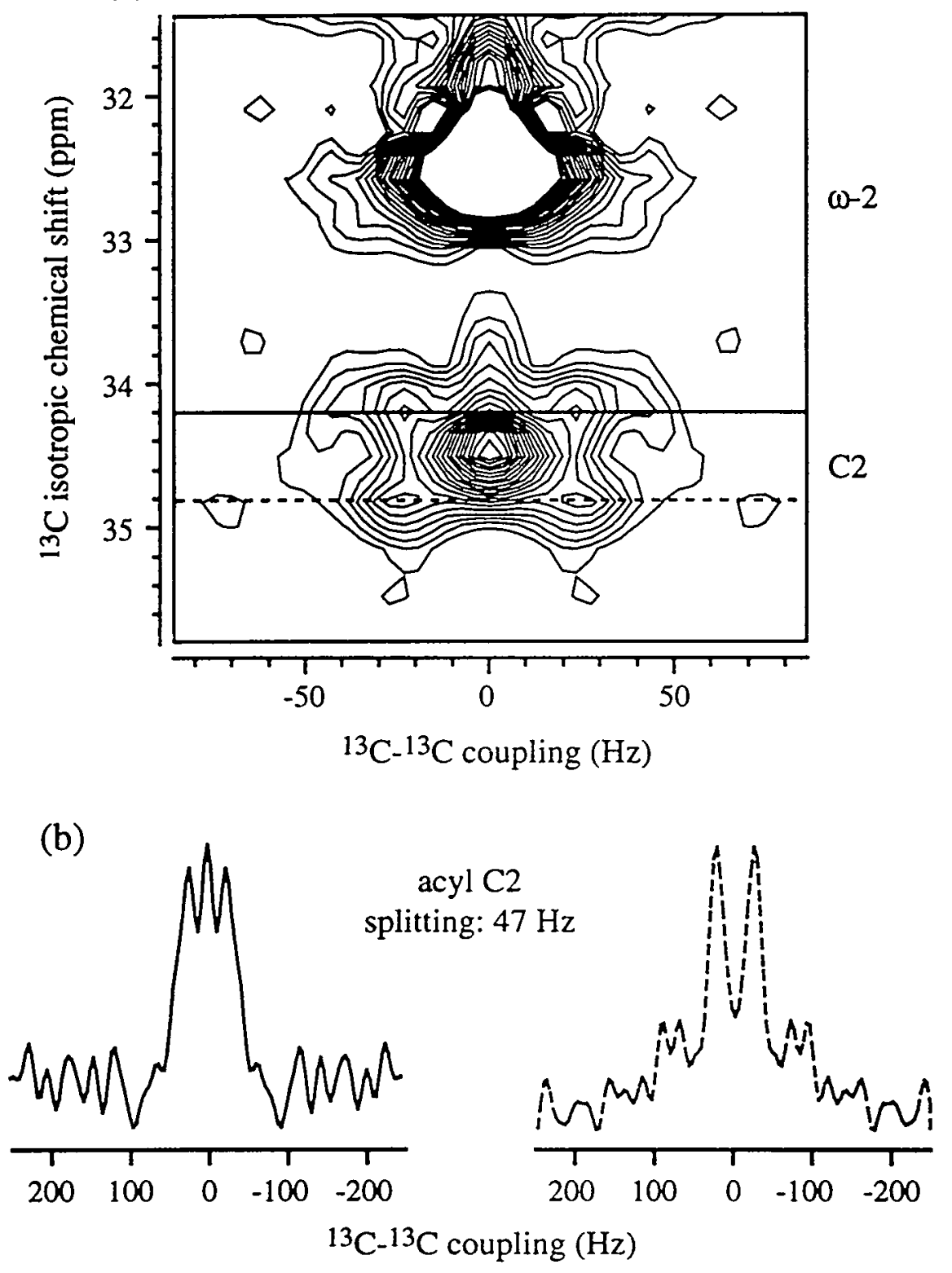

Figure 5.21 ${ }^{13} \mathrm{CO}$-DMPC SLF spectrum obtained under SAS with dipolar scaling factor $P_{2}\left(\cos 69^{\circ}\right)=-0.3$. (a) $2 \mathrm{D}$ spectrum in the $\mathrm{C} 2$ region. (b) Dipolar coupling cross sections from the $\mathrm{C} 2$ butterfly pattern. 

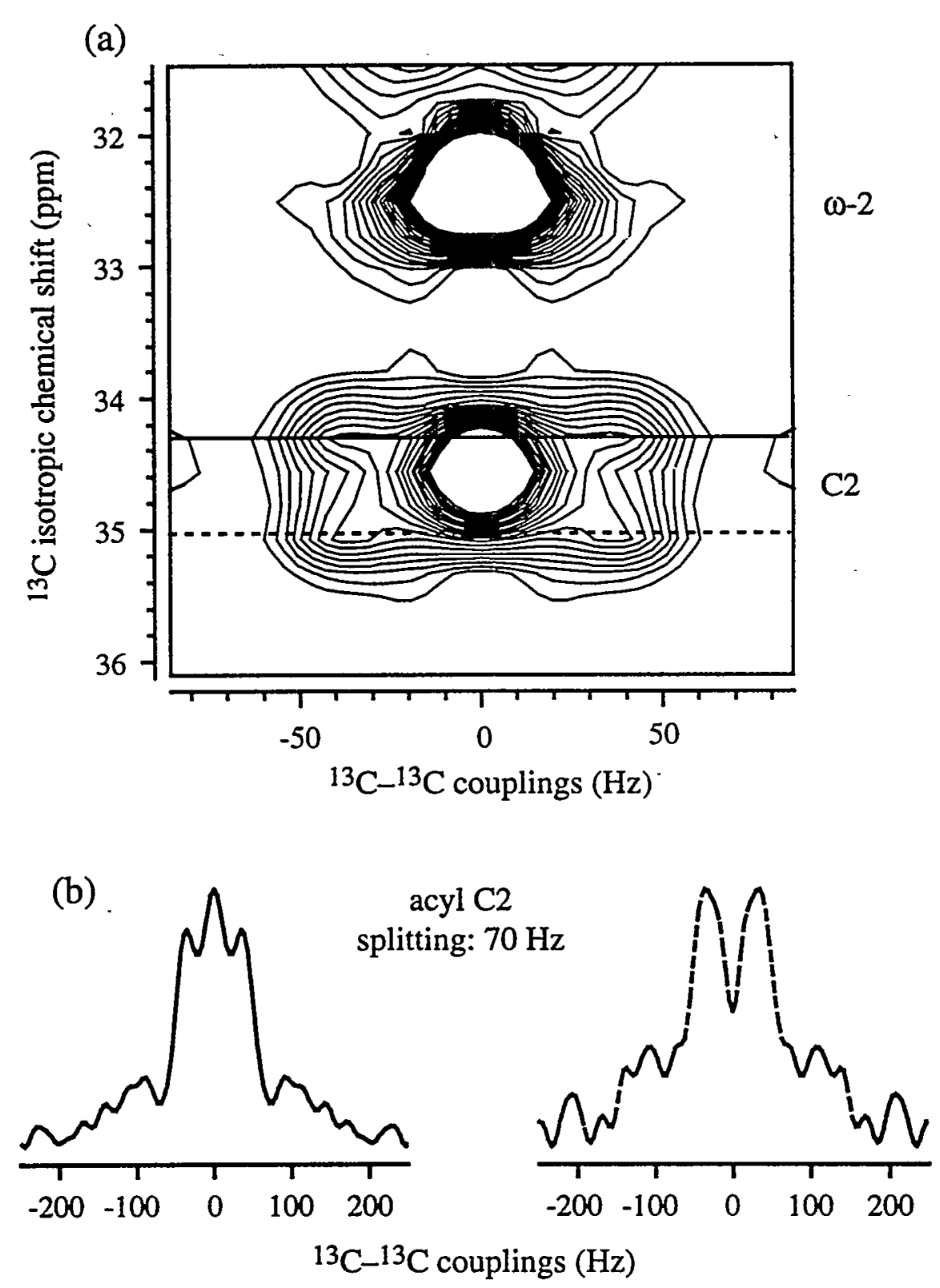

Figure 5.22 ${ }^{13} \mathrm{CO} 2$-DMPC SLF spectrum obtained under SAS with dipolar scaling factor $P_{2}\left(\cos 43^{\circ}\right)=+0.3$. (a) $2 \mathrm{D}$ spectrum in the $\mathrm{C} 2$ region. (b) Dipolar coupling cross sections from the $\mathrm{C} 2$ butterfly pattern. 
Combining the results from the two spectra, we find the $\mathrm{CO} 2-\mathrm{C} 2$ coupling to be $-40 \pm 22 \mathrm{~Hz}$, corresponding to a $\mathrm{CO} 2-\mathrm{C} 2$ order parameter of $+0.019 \pm 0.010$. Using distance and orientation arguments, we have expected to see $\mathrm{CO} 2-\mathrm{G} i(i=1,2,3)$ dipolar splittings as well. However, no such splittings can be observed in the spectra. This may be due to the low sensitivity of the glycerol ${ }^{13} \mathrm{C}$ signals. As the most rigid segments in the molecule, the glycerol sites experience the largest $\mathrm{C}-\mathrm{H}$ dipolar couplings, which broaden their spectral lines.

\subsection{Determination of Dipolar Coupling Signs by DISTINCT NMR}

So far, we presented the measurement of the magnitudes of dipolar couplings for various spin pairs. However, the signs of these dipolar couplings are not obtained except for the directly-bonded $\mathrm{C}-\mathrm{H}$ and $\mathrm{C}-\mathrm{C}$ couplings. The reason for the general lack of sign information lies in the symmetry of the dipolar spectrum, be it a Pake pattern for powder samples or a resolved doublet for oriented samples. As we discussed in chapter 4 , the dipolar coupling sign contains information on the preferential orientation of the internuclear vectors, and thus the segments, with the motional axis in uniaxially mobile molecules. For instance, $\mathrm{C}-\mathrm{H}$ bonds oriented preferentially normal to the director exhibit a negative order parameter $S_{C H}=\bar{\delta}_{D} / \delta_{D}$. (This corresponds to a positive $\bar{\delta}_{D}$, as the rigid-lattice dipolar coupling $\delta_{D}$ is negative ${ }^{29}$ by definition.)

We demonstrated in section 5.2 that it is possible to obtain the signs of directlybonded dipolar couplings by comparing the splittings in two PDLF spectra taken at different spinning angles. In the following, we show that the dipolar coupling signs can be observed more directly as the polarities (positive or negative) of peak amplitudes in a $1 \mathrm{D}$ SAS spectrum. This new technique, called DISTINCT for DIpolar Sine Term by INdirectCoupling Transforma-tion, uses $J$ couplings to make sign-dependent dipolar coherence observable. In the following, we describe the DISTINCT pulse sequence and the corresponding time evolution of the density operator in detail. We show both theoretically 
and experimentally that the intensities of the ${ }^{13} \mathrm{C}$ signals in the 1D DISTINCT spectra are mainly determined by the $90^{\circ}$ singularities of the dipolar powder patterns. Extending the technique to $2 \mathrm{D}$, we can determine unambiguously the signs of motionally-averaged dipolar coupling constants. In practice, we find that the sign determination is most reliable when the effective dipolar coupling is larger than the $J$ coupling. Our original DISTINCT experiment involves PDLF evolution of the dipolar coupling. A variation of the experiment, which involves dipolar evolution on the heteronucleus (e.g. $\left.{ }^{13} \mathrm{C}\right)$, will also be discussed.

\section{DISTINCT with Proton-Detected-Local-Field Evolution}

The DISTINCT pulse sequence is displayed in Figure 5.23. In the evolution period, the sample is spun about an axis inclined at angle $\theta$ to the magnetic field, where $\theta \neq 54.7^{\circ}$. Thus all anisotropic interactions in $t_{1}$ are scaled by $P_{2}(\cos \theta)=\frac{1}{2}\left(3 \cos ^{2} \theta-1\right)$. Proton magnetization, flipped to the $x y$ plane of the rotating frame by a $90^{\circ}$ excitation pulse, evolves under $\mathrm{C}-\mathrm{H}$ heteronuclear interactions. These include the $\mathrm{C}-\mathrm{H}$ dipolar coupling, scaled by $P_{2}(\cos \theta)$ and the MREV-8 scaling factor $s_{m}$, and the isotropic $J$ coupling. The anisotropic part of the $J$ coupling can be neglected relative to the dipolar coupling. ${ }^{30}$ Homonuclear $\mathrm{H}-\mathrm{H}$ interaction is suppressed by a MREV-8 multiple-pulse sequence, and the ${ }^{1} \mathrm{H}$ chemical shift is refocused by a ${ }^{1} \mathrm{H} 180^{\circ}$ pulse in the middle of the $t_{1}$ period. A simultaneous ${ }^{13} \mathrm{C} 180^{\circ}$ pulse serves to restore the $\mathrm{C}-\mathrm{H}$ couplings. The active Hamiltonian during the evolution period is

$$
H_{C H}=\omega_{C H} \cdot 2 S_{z} \frac{1}{\sqrt{2}}\left(I_{z}+I_{x}\right)
$$

The frequency $\omega_{C H}$ is defined as $s_{m} \cdot\left(P_{2}(\cos \theta) \cdot \bar{\omega}_{d}+\pi J\right)$, where $\bar{\omega}_{d}$ and $J$ represent the motionally-averaged dipolar coupling and the isotropic $J$ coupling between the directlybonded ${ }^{13} \mathrm{C}$ and ${ }^{1} \mathrm{H}$ spins. 

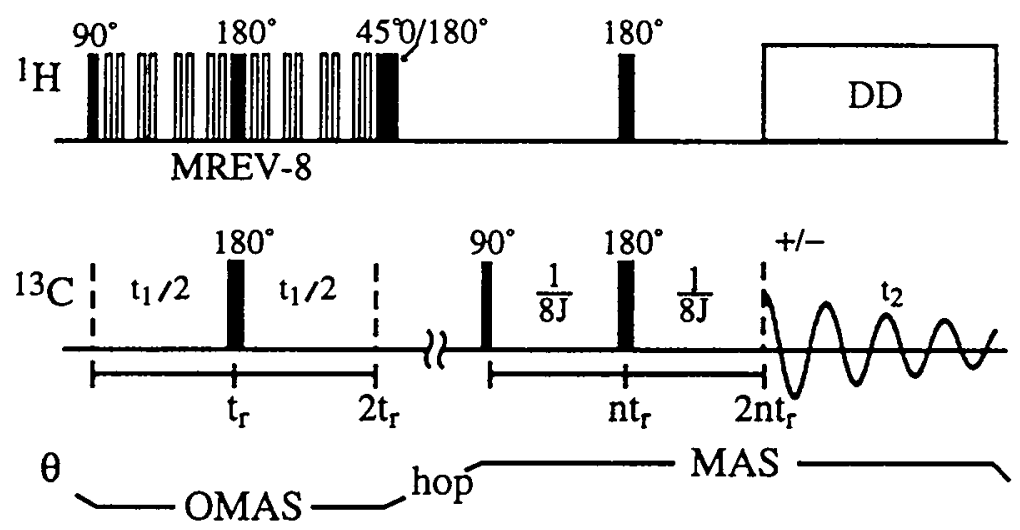

Figure 5.23 DISTiNCT pulse sequence.

The ${ }^{1} \mathrm{H}$ magnetization $I_{y}$ evolves under the Hamiltonian (5.2) into

$$
I_{y} \rightarrow I_{y} \cos \left(\omega_{C H_{1} t_{1}}\right)+2 S_{z} \frac{1}{\sqrt{2}}\left(-I_{x}+I_{z}\right) \sin \left(\omega_{C H_{1} t_{1}}\right) .
$$

At the end of the evolution period, a $45^{\circ}-y{ }^{1} \mathrm{H}$ pulse converts the sine-modulated term into two-spin order $2 S_{z} I_{z} \sin \left(\omega_{C H} t_{1}\right)$, whereas the $I_{y}$ term remains invariant. In the following mixing period of about $50 \mathrm{~ms}$, the rotor axis is hopped to the magic angle, and the transverse magnetization $I_{y}$ dephases while the two-spin order survives. Subsequently, a $90^{\circ}-{ }^{13} \mathrm{C}$ pulse is applied to create antiphase heteronuclear coherence

$$
2 S_{x} I_{z} \sin \left(\omega_{C H} t_{1}\right)=2 S_{x}\left(I_{z}^{a}+I_{z}^{b}+I_{z}^{c}\right) \sin \left(\omega_{C H} t_{1}\right)
$$

from the two-spin order. In the next time period of duration $\tau=2 n \cdot t_{r}$, all spin interactions are removed except for the isotropic $J$ coupling. The chemical shift interaction is refocused by ${ }^{1} \mathrm{H}$ and ${ }^{13} \mathrm{C} 180^{\circ}$ pulses at $\tau / 2$, while the $\mathrm{C}-\mathrm{H}$ and the $\mathrm{H}-\mathrm{H}$ dipolar couplings are averaged out at full MAS rotor periods $n \cdot t_{r}$ due to the uniaxial motion of the lipid molecules. The $J$ interaction transforms the heteronuclear coherence $2 S_{x} I_{z}$ to observable 
${ }^{13} \mathrm{C}$ magnetization $S_{y}$, which is then detected in the presence of proton decoupling. The last transformation for a $\mathrm{CH}$ segment is given by

$$
2 S_{x} I_{z} \rightarrow 2 S_{x} I_{z} \cos (\pi J \tau)+S_{y} \sin (\pi J \tau)
$$

In general, in a $\mathrm{CH}_{\mathrm{n}}(\mathrm{n}=1,2,3)$ group where the ${ }^{13} \mathrm{C}$ nucleus evolves in the total fields of the $\mathrm{n}$ protons, the finally detected density operator is

$$
S_{y} \sin \left(\omega_{C H} t_{1}\right) \sin (n \pi J \tau)
$$

for the methine $(n=1)$ and the methylene groups $(n=2)$. For the methyl groups $(n=3)$, the signal is (appendix A)

$$
S_{y} \sin \left(\omega_{C H_{1}}\right) \cdot\left[\sin (3 \pi J \tau)+\sin ^{3}(\pi J \tau)\right]
$$

The length $\tau$ of the $J$ coupling period is chosen to maximize the signal intensities. Since $\mathrm{CH}_{2}$ groups are predominant in phosphocholine lipids, $\tau=\frac{1}{4 J}$ so that $\sin (2 \pi J \tau)=1$. Therefore, the detected ${ }^{13} \mathrm{C}$ MAS signals are modulated by the sine of the heteronuclear $\mathrm{C}-\mathrm{H}$ couplings,

$$
f\left(t_{1}, t_{2}\right) \propto \sin \left(\omega_{C H} t_{1}\right) e^{i \omega_{c s} t_{2}}
$$

At sufficiently short $t_{1}$, due to

$$
\sin \left(\omega_{C H}^{-} t_{1}\right) \approx \omega_{C H} t_{1},
$$


the sign of the heteronuclear coupling $\omega_{C H}$ is manifested as the sign of the ${ }^{13} \mathrm{C}$ signal. This sign of $\omega_{C H}$ can be converted directly into the sign of the dipolar coupling constant $\bar{\omega}_{d}$, provided that $P_{2}(\cos \theta) \cdot \bar{\omega}_{d}>\pi J$.

\section{Effects of Powder Average}

So far, we have neglected the fact that the liquid-crystalline phospholipids used in our experiments are powder samples consisting of randomly oriented bilayers. Because dipolar coupling depends on the orientation of the directors relative to the rotor axis, the dipolar spectrum is a symmetric powder pattern ${ }^{31}$ with both positive and negative frequencies. One might argue that such a distribution of frequencies makes the sign of the C-H coupling $\omega_{C H}$ indeterminate, since the averaged dipolar coupling $\bar{\omega}_{d}$ depends on the bilayer orientation according to

$$
\begin{aligned}
\bar{\omega}_{d} & =\frac{1}{2} \delta_{d}\left\langle 3 \cos ^{2} \Theta-1\right\rangle \cdot \frac{1}{2}\left(3 \cos ^{2} \beta-1\right) \\
& =\bar{\delta}_{d} \cdot \frac{1}{2}\left(3 \cos ^{2} \beta-1\right)
\end{aligned}
$$

Here $\Theta$ denotes the angle between the internuclear vector and the director, and $\beta$ is the angle between the director and the magnetic field. Fortunately, this bilayer orientation distribution does not affect the determination of the dipolar coupling sign seriously. A sine-modulated dipolar powder spectrum (Figure 5.24a) is dominated by the singularities at

$$
\pm \omega_{C H}\left(\beta=90^{\circ}\right)= \pm s_{m}\left(-\frac{1}{2} \bar{\delta}_{d} \cdot P_{2}(\cos \theta)+\pi J\right)
$$

which result from bilayers whose directors are normal to the $B_{0}$ field. This means that the ${ }^{13} \mathrm{C}$ signals in $1 \mathrm{D}$ DISTINCT spectra should be modulated mainly by these powder

maxima, and reflect the opposite sign of $\bar{\delta}_{d}$. The sine dipolar pattern is antisymmetric and 
can be described as $P(\omega-\pi J)-P(-\omega+\pi J)$, where $P(\omega)$ is the lineshape function of a single transition. The conventional cosine Pake pattern (Figure 5.24b) is symmetric with a lineshape of $P(\omega-\pi J)+P(-\omega+\pi J)$. Due to the odd symmetry, the intensities in the sine spectrum change signs according to the sign of $\bar{\delta}_{d}$, provided that the spinning-scaled dipolar coupling exceeds the $J$ coupling.

To verify that the dipolar powder maxima determine the ${ }^{13} \mathrm{C}$ MAS intensities, we can increment the $t_{1}$ of the 1D DISTINCT pulse sequence to obtain a $2 \mathrm{D}$ spectrum, so that the $\omega_{1}$ dimension explicitly shows the antiphase $\mathrm{C}-\mathrm{H}$ coupling spectra. However, the expected antiphase powder lineshape requires more detailed analysis, since it depends on the signs and the relative sizes of the $J$ coupling to the dipolar coupling.
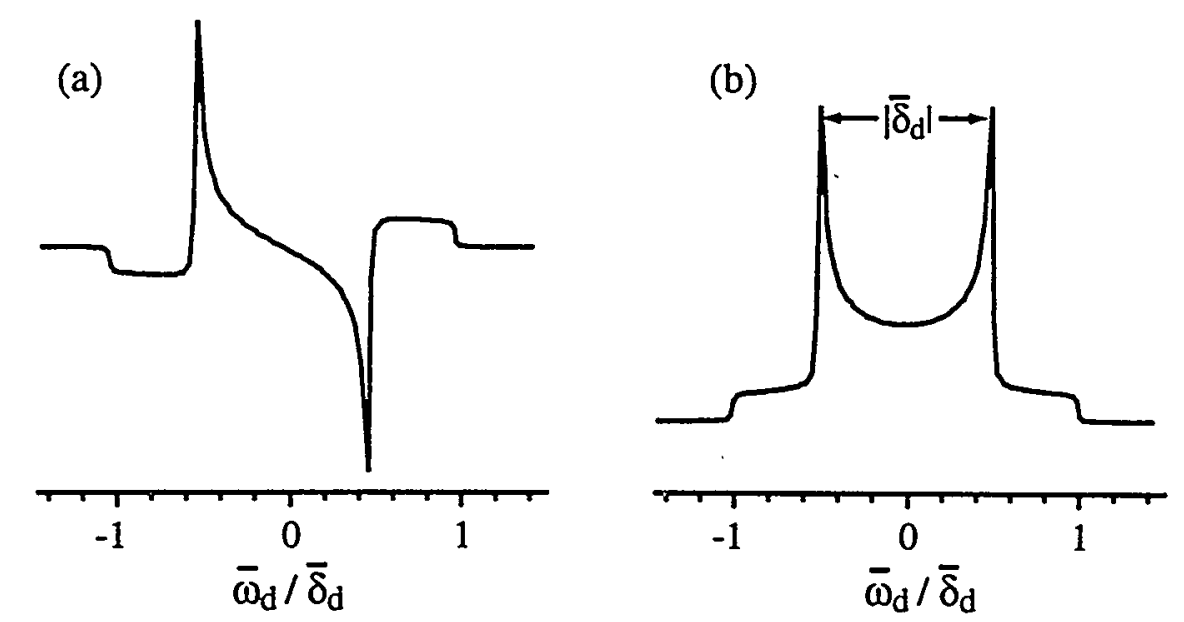

Figure 5.24 Simulated dipolar spectra. (a) Sine spectrum. (b) cosine Pake pattern.

Figure 5.25 shows the simulation of antiphase dipolar- $J$ lineshapes as a function of the ratio between the effective dipolar coupling and the isotropic $J$ coupling, $P_{2}(\cos \theta) \delta_{d}$ $/ \pi J$. The sign of the $J$ coupling is set to be positive, as appropriate for one-bond C-H $J$ couplings. The simulated spectra consist of both halves of a Pake pattern, $\pm P( \pm(\omega-\pi J))$, each with the width of $1.5 \cdot P_{2}(\cos \beta) \bar{\delta}_{d}$. The centers of gravity of the half Pake patterns are located at $\pm \pi J$. The sign of the two spectral maxima, which are the most easily detected 
features, changes at parameter values of $P_{2}(\cos \theta) \cdot \bar{\delta}_{d}=\pi J$. As expected, for dipolar couplings smaller than the $J$ coupling, the sign of the singularities is that of an antiphase $J$ doublet. However, even for these small dipolar couplings, the details of the powder lineshapes are different. For example, for $P_{2} \bar{\delta}_{D} / \pi J= \pm 1 / 4$, the positions of the maxima relative to the low edges of the powder patterns are opposite. With sufficient resolution, it is possible to determine the signs and magnitudes of $\bar{\delta}_{d}$ from these characteristic lineshapes.

With these considerations in mind, we now analyze a 2D DISTINCT spectrum to extract the sign of dipolar couplings. Such a spectrum of lecithin, with a spinning scaling factor of $P_{2}(\cos \theta)=-0.2$, is shown in Figure 5.26. The $\mathrm{C}-\mathrm{H}$ dipolar couplings, manifested as antisymmetric spectra centered around $\omega_{1}=0$, are separated according to the isotropic ${ }^{13} \mathrm{C}$ chemical shifts in the $\omega_{2}$ dimension. The positive and negative intensities of the dipolar powder patterns are indicated by dark and gray contours, respectively. It can be seen that each dipolar cross section is dominated by two maximum intensities, which correspond to the directors that are normal to the $B_{0}$ field. The antiphase patterns of most sites exhibit the same polarity except for the $\mathrm{C} \alpha$ site. This indicates that all sites except for $\mathrm{C} \alpha$ have the same sign for the heteronuclear coupling $\omega_{C H}$. The structural implication for the sign difference of the $\mathrm{C} \alpha-\mathrm{H} \alpha$ coupling will be discussed later.

The presence of intensity maxima in the above 2D DISTINCT spectrum strongly suggests that the ${ }^{13} \mathrm{C}$ signals in the $1 D$ DISTINCT spectra should reflect the sign of dipolar coupling at the powder pattern singularities. For a more complete analysis of the $1 \mathrm{D}$ DISTINCT intensities, we also need to examine the time oscillations of the antiphase heteronuclear spectra, since the ${ }^{13} \mathrm{C}$ signals are directly modulated by $\sin \left(\omega \mathrm{CH} \mathrm{t}_{1}\right)$ according to equation (5.8). In addition, the effect of the positive ${ }^{1} J_{C H}$ on the dipolar sign determination must be included. 


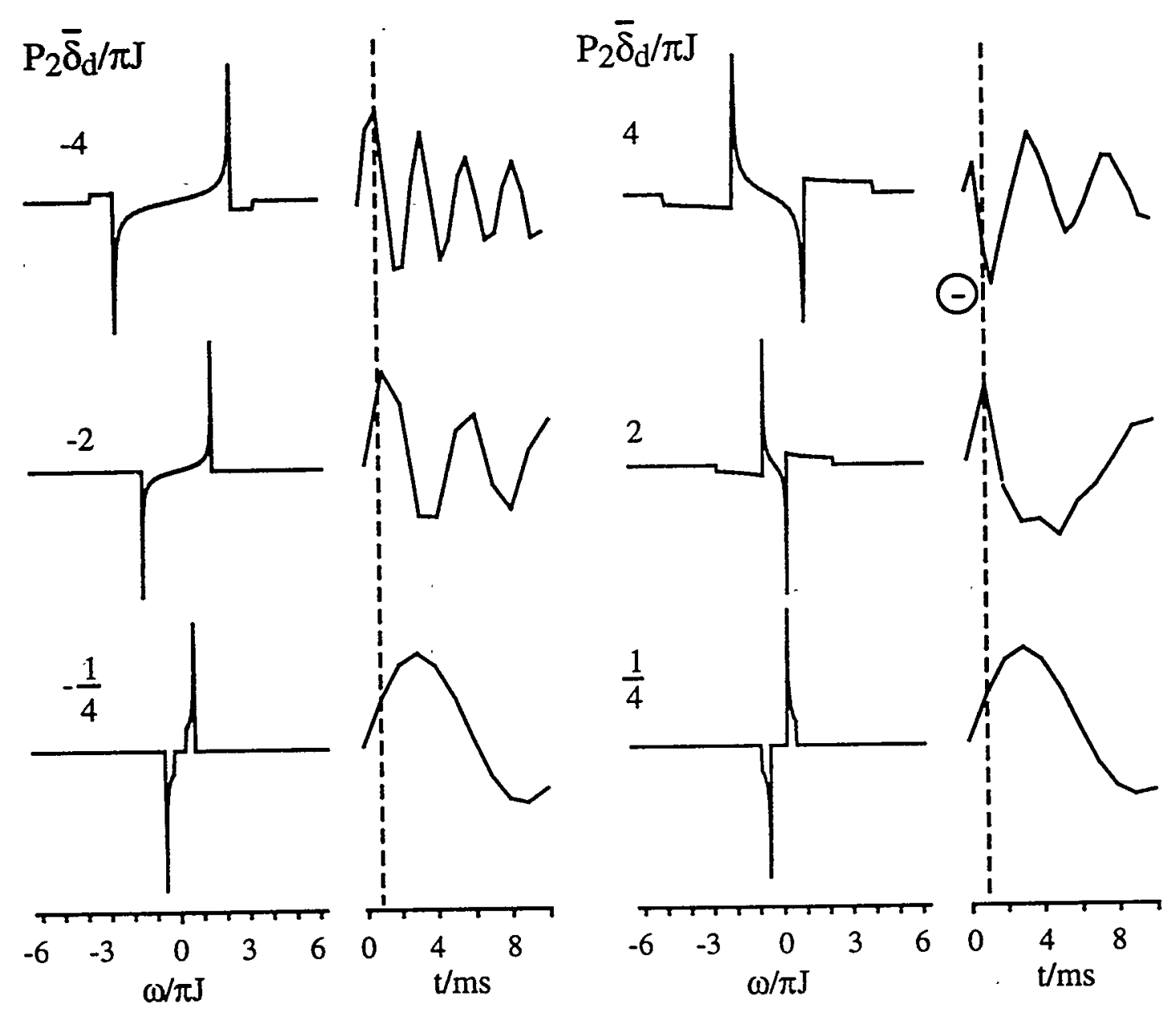

Figure 5.25 Calculated antiphase dipolar- $J$ spectra as a function of $P_{2}(\cos \theta) \delta_{d} / \pi J$.

The corresponding time-domain oscillations are shown. 


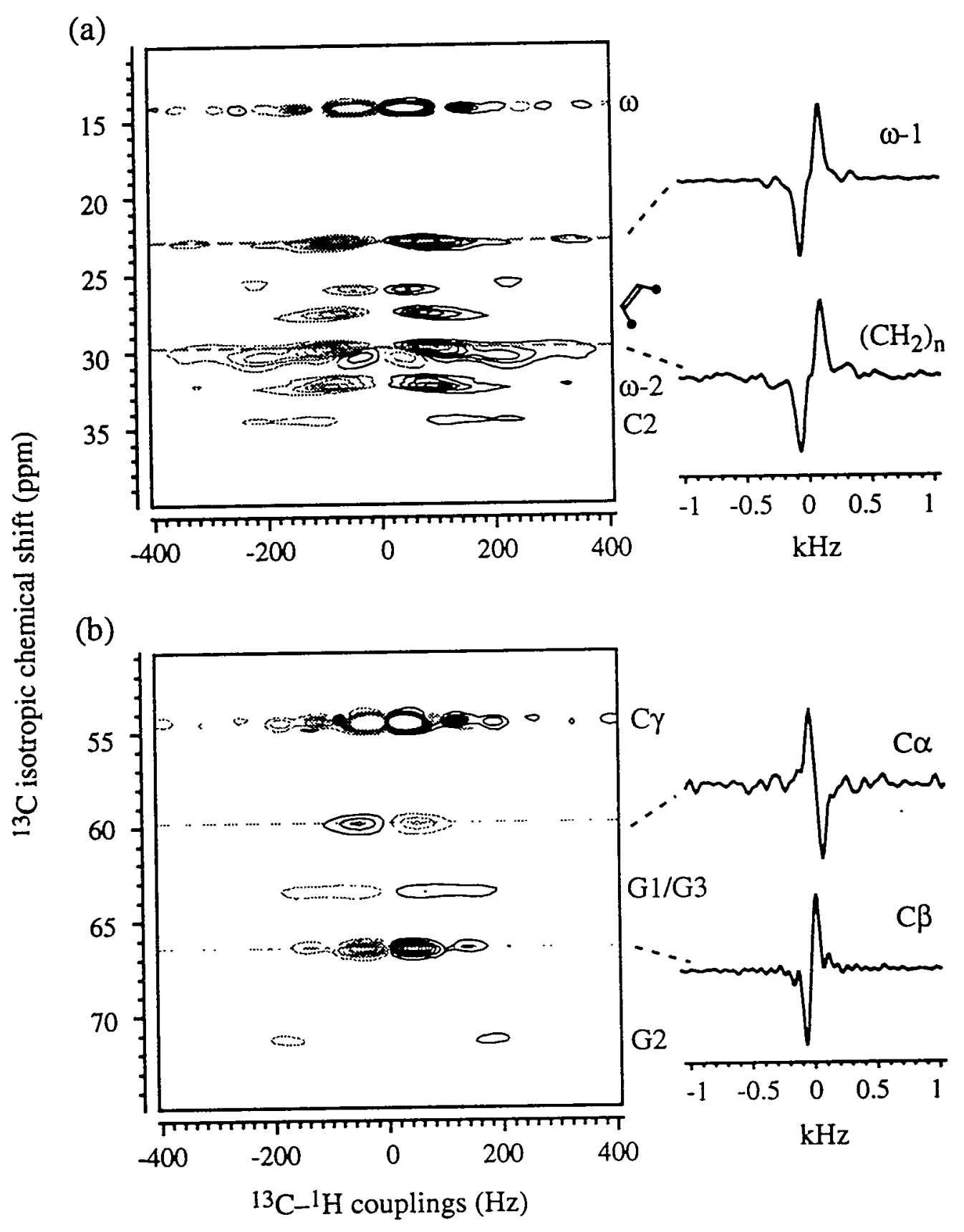

Figure 5.26 $\mathrm{L}_{\alpha}$-lecithin 2D DISTINCT spectrum. (a) Acyl chain region. (b) Headgroup and glycerol regions. Spinning scaling factor: $P_{2}(\cos \theta)=-0.2$. Measuring time: 10 hours. 
The sine time signals of the antiphase spectra for a series of relative ratios of the dipolar and $J$ couplings are shown in Figure 5.25. The oscillatory behavior at long and intermediate times is clearly dominated by the oscillation of the $90^{\circ}$ frequencies. The behavior at short and intermediate times, which are of most interest to 1D DISTINCT experiments, can be written as

$$
\begin{aligned}
f_{s}(t) & =\int P(\omega) \sin (\omega) d \omega \\
& =t \int P(\omega) \omega d \omega-\frac{t^{3}}{6} \int P(\omega) \omega^{3} d \omega+O\left(t^{5}\right) \\
& =-\frac{t^{3}}{6} M_{3}+O\left(t^{5}\right)
\end{aligned}
$$

for the case without $J$ coupling. In this equation, the first moment $\int P(\omega) \omega d \omega$ vanishes because it corresponds to the center of gravity of $P(\omega)$, and the third moment $M_{3}$ has the same sign as $\bar{\delta}_{d}$. Thus the time signal is sensitive to the sign of $\bar{\delta}_{d}$. For the case with $J$ coupling, the oscillation at short times becomes

$$
f_{s}(t)=t \cdot \pi J-\frac{t^{3}}{6} M_{3}+O\left(t^{5}\right)
$$

This means that when the $P_{2}$-scaled dipolar coupling is comparable to the $J$ coupling, i.e. when the first term in (5.13) is greater than the second, the short-time signal fails to reflect the sign of $\bar{\delta}_{d}$. As indicated in Figure 5.25, the initial decays of the time signals with $P_{2}(\cos \theta) \bar{\delta}_{d} \gg \pi J$ (e.g. ratios of \pm 4 ) are sensitive to the $90^{\circ}$ singularities of the dipolar spectra. The signal at a fixed initial time point, $1 \mathrm{~ms}$, changes sign with the sign of the dipolar coupling. However, when $P_{2}(\cos \theta) \cdot \bar{\delta}_{d} \leq 2 \pi J$ (e.g. ratios of \pm 2 and \pm 0.25 ), the time signal remains positive at the beginning, because it does not oscillate fast enough to reflect the $90^{\circ}$ intensities of the powder pattern. Therefore, the 1D DISTINCT technique only detects the sign of relatively large dipolar couplings. Fortunately, in $\mathrm{L}_{\alpha}$ - 
phosphocholine, the directly-bonded $\mathrm{C}-\mathrm{H}$ dipolar couplings in most headgroup and glycerol sites are much larger than the isotropic $J$ coupling, according to the ${ }^{2} \mathrm{H}$ NMR data.

Figure 5.27 shows two 1D DISTINCT spectra of lecithin, taken with $P_{2}(\cos \theta)= \pm 0.2$ and with a fixed dipolar evolution time of $960 \mu \mathrm{s}$, which corresponds to two rotor periods. In the spectra, the positive reference for the signs is provided by the $\mathrm{CH}_{3}$ ( $\omega$ and $\gamma$ ) signals, ${ }^{8}$ as they are dominated by the positive ${ }^{1} J_{C H}$, which are +120 to $+150 \mathrm{~Hz}$ as measured by a MAS experiment in the absence of proton decoupling. It follows that negative intensities in either spectrum are definite indications that the $P_{2}$-scaled dipolar coupling constant has the same sign as the $J$ coupling, and that $P_{2}(\cos \theta) \cdot \bar{\delta}_{d} \gg \pi J$. For example, at $P_{2}=+0.2$ (Figure 5.27a), the acyl chain ${ }^{13} \mathrm{C}$ signals are negative, thus the $\bar{\delta}_{d}$ of acyl sites are positive. Since the rigid-limit dipolar constant $\delta_{d}$ is a priori negative, the acyl carbons must have negative order parameters $S_{C H}=\frac{1}{2}\left\langle 3 \cos ^{2} \Theta-1\right\rangle$. This is consistent with the parallel orientation of the acyl chains with the bilayer normal, since the $\mathrm{C}-\mathrm{H}$ vectors are then preferentially normal to the director and $\frac{1}{2}\left\langle 3 \cos ^{2} \Theta-1\right\rangle \approx-\frac{1}{2}<0$. In contrast to most acyl and glycerol carbon sites, the $\mathrm{C} \alpha$ signal in the spectrum with $P_{2}=-0.2$ (Figure 5.27b) is negative. Carrying out the sign analysis, we find that $\mathrm{C} \alpha$ is the cnly segment with a large positive $S_{C H}$, or a large negative $\bar{\delta}_{d}$. This sign anomaly demonstrates an unusual orientation of the $\mathrm{C} \alpha$ group, and is consistent with the bend of the headgroup at the phosphate junction. ${ }^{32}$ For the $C \beta$ site, DISTINCT spectra with $P_{2}= \pm 0.4$ (not shown) exhibit only positive peaks, indicating that the $\mathrm{C} \beta-\mathrm{H} \beta$ coupling is significantly smaller $\left(S_{C H}<0.02\right)$ than expected from ${ }^{2} \mathrm{H}$ NMR $(0.045)$ in fully hydrated bilayers. ${ }^{7}$ This variation in order parameter might be attributed to the lower hydration level of our sample.

Comparing the merits of $1 \mathrm{D}$ and 2D DISTINCT spectroscopy, it is clear that the $1 \mathrm{D}$ technique allows a faster examination of the dipolar coupling sign. However, the interpretation of the 1D spectra can be ambiguous due to the complex time evolution for a powder sample, especially when the dipolar couplings are smaller than or comparable to the 
$J$ coupling. Therefore, the 2D version of DISTINCT, while more time-consuming, is important for an unambiguous determination of the signs of dipolar couplings.

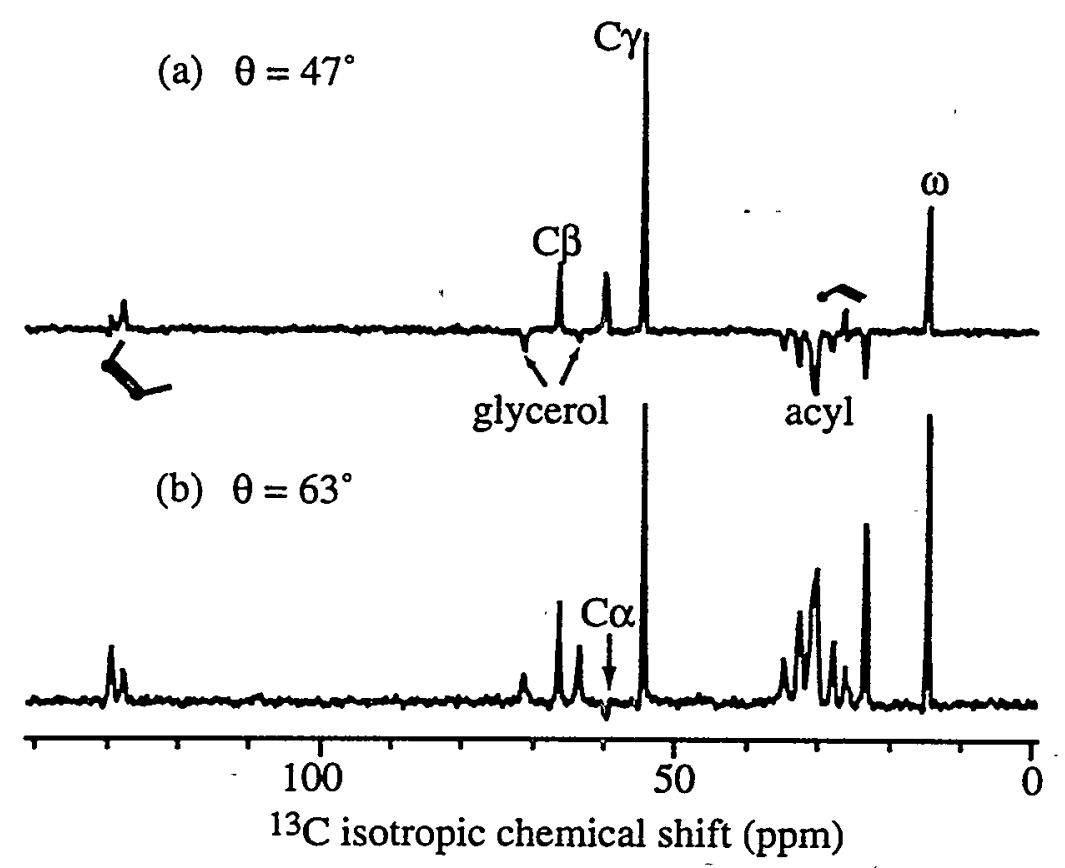

Figure 5.27 $\mathrm{L}_{\alpha}$-lecithin ID DISTINCT spectra beginning with ${ }^{1} \mathrm{H}$ magnetization. (a) $P_{2}=+0.2$. (b) $P_{2}=-0.2$. ${ }^{13} \mathrm{C}$ resonances are assigned according to Husted et al. ${ }^{8}$ Measuring time: 8 hours for each spectrum.

\section{DISTINCT with Carbon-Detected-Local-Field Evolution}

The DISTINCT technique can also be implemented beginning with ${ }^{13} \mathrm{C}$ magnetization, which evolves in the local fields of the surrounding protons as in conventional SLF experiments. ${ }^{25,33}$ Since at least $n$ protons couple to the ${ }^{13} \mathrm{C}$ spin in a $\mathrm{CH}_{\mathrm{n}}$ segment, the ${ }^{13} \mathrm{C}$ MAS signal is modulated by the sine of the sum of the $\mathrm{C}-\mathrm{H}$ dipolar couplings. This can be derived by considering the Hamiltonian of $\mathrm{n}$ protons $a, b, \ldots$ interacting with ${ }^{13} \mathrm{C}$,

$$
H_{C H}=2 S_{z}\left(\omega_{C H}^{a} I_{z}^{a}+\omega_{C H}^{b} I_{z}^{b}+\ldots\right)
$$


Since the coupling terms for different spins $a, b, \ldots$ commute, the time evolution of the density operator can be calculated sequentially. ${ }^{16}$ For a $\mathrm{CH}_{2}$ group, the density operator evolves under the dipolar Hamiltonian as

$$
\begin{aligned}
S_{y} \stackrel{t_{1}}{\longrightarrow} & 2 S_{x} I_{z}^{a} \sin \left(\omega_{C H}^{a} t_{1}\right) \cos \left(\omega_{C H}^{b} t_{1}\right) \\
+ & 2 S_{y} I_{z}^{b} \cos \left(\omega_{C H}^{a} t_{1}\right) \sin \left(\omega_{C H}^{b} t_{1}\right) \\
\stackrel{\tau}{\longrightarrow} & S_{y} \cdot\left[\sin \left(\omega_{C H}^{a} t_{1}\right) \cos \left(\omega_{C H_{1}}^{b}\right)+\cos \left(\omega_{C H^{\prime}}^{a}\right) \sin \left(\omega_{C H^{t_{1}}}^{b}\right)\right] \\
& \cdot \sin (\pi J \tau) \cos (\pi J \tau)
\end{aligned}
$$

where $\omega_{C H}^{a}$ and $\omega_{C H}^{b}$ are the heteronuclear couplings of ${ }^{13} \mathrm{C}$ to $\mathrm{H} a$ and $\mathrm{H} b$, respectively. The signal (5.15) has the required invariance with respect to an interchange of $\mathrm{H} a$ and $\mathrm{H} b$. The ${ }^{13} \mathrm{C}$ signal detected after $J$ evolution under MAS can be rewritten as

$$
S_{y} \sin \left[s_{m}\left(P_{2}(\cos \theta) \cdot\left(\omega_{d}^{a}+\omega_{d}^{b}\right)+2 \pi J\right) t_{1}\right] \cdot \frac{1}{2} \sin (2 \pi J \tau)
$$

As before, in order to obtain maximal ${ }^{13} \mathrm{C}$ signals, $\tau$ is chosen such that $\sin (2 \pi J \tau)=1$.

Two SLF-DISTINCT spectra with scaling factors of $P_{2}(\cos \theta)= \pm 0.2$ are shown in Figure 5.28. The signs of all peaks are consistent with those obtained in the corresponding PDLF-DISTINCT spectrum (Figure 5.27). ${ }^{2} \mathrm{H}$ NMR and our 2D SAS PDLF experiments have shown that the two $\mathrm{C}-\mathrm{H}$ couplings $\left(\omega_{d}^{a}\right.$ and $\omega_{d}^{b}$ ) are degenerate in most $\mathrm{CH}_{2}$ groups of lecithin. As a result, the ${ }^{13} \mathrm{C}$ signals are modulated by approximately twice the $\mathrm{C}-\mathrm{H}$ dipolar coupling as that in the PDLF-DISTINCT spectrum. This difference in modulation frequency gives rise to different relative intensities in the SLF- and the PDLF-DISTINCT spectra. For instance, at $P_{2}(\cos \theta)=+0.2$ (Figure 5.28a), the $\gamma$ signal is $\sim 8$ times higher than the main $\left(\mathrm{CH}_{2}\right)_{\mathrm{n}}$ peak while the ratio is only about 5 in the corresponding PDLF spectrum (Figure 5.27a). 


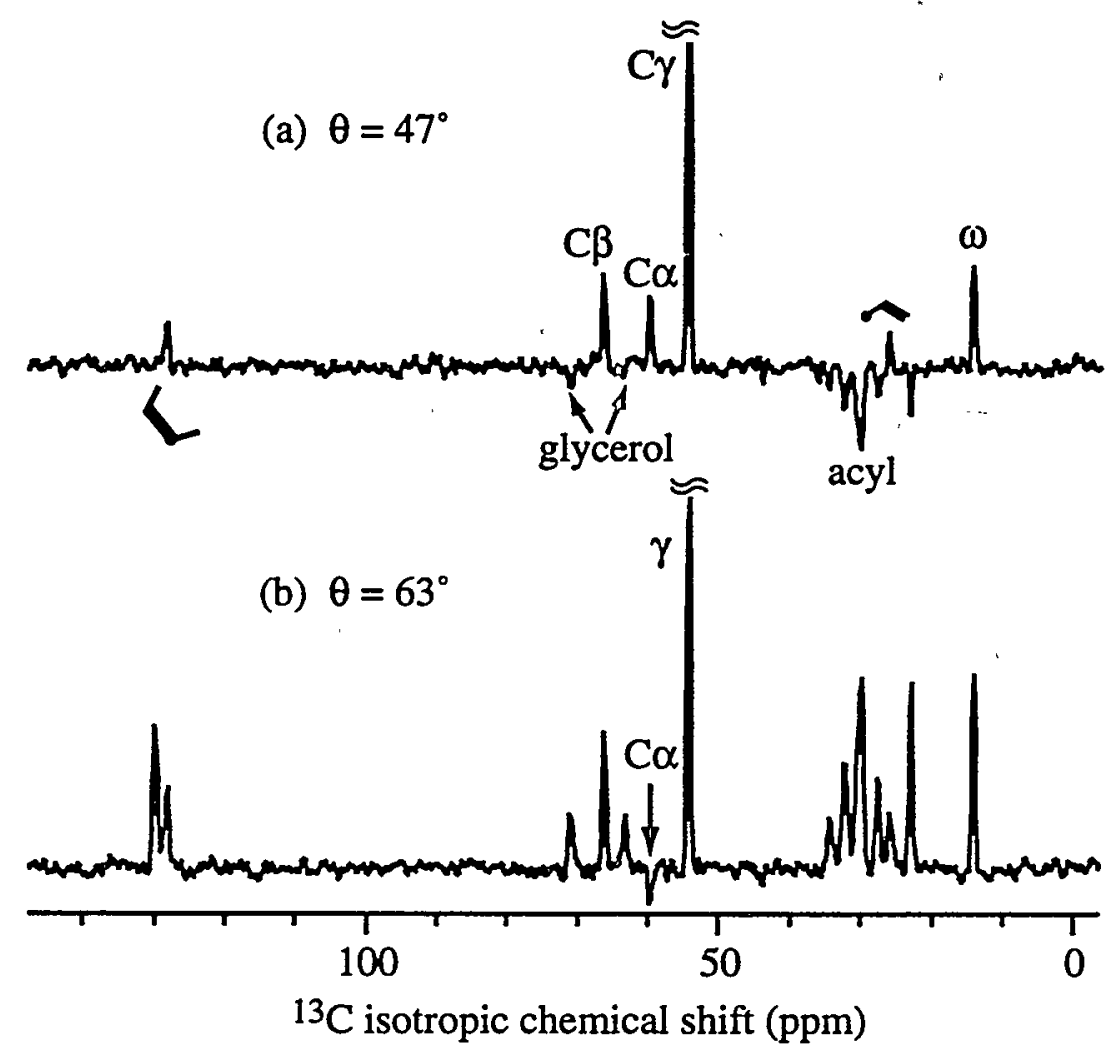

Figure 5.28 $\mathrm{L}_{\alpha}$-lecithin 1D DISTINCT spectrum beginning with ${ }^{13} \mathrm{C}$ magnetization. (a) $P_{2}=+0.2$. (b) $P_{2}=-0.2$. Measuring time: 8 hours for each spectrum.

Clearly, the ${ }^{13} \mathrm{C}$-detected DISTINCT spectra have lower sensitivities. This is mostly due to the additional scaling factor of $1 / 2$ in equation (5.16) compared to the PDLF signal in equation (5.6). The sensitivities are similar for the methine signals $(-130 \mathrm{ppm})$ in the SLF and PDLF spectra, because a methine group has a single proton and evolves identically under SLF and PDLF conditions. 


\section{9 ${ }^{13} \mathrm{C}$ Chemical Shift Anisotropies}

So far, we measured various dipolar couplings in $\mathrm{L}_{\alpha}$-phosphocholine to constrain the segmental orientations in the molecule. In general, all anisotropic NMR interactions, such as chemical shift and quadrupolar couplings, can serve as conformational constraints. The use of the averaged CSAs requires the knowledge of the chemical shift tensor orientation in the molecular frame, which is often not directly available in the segment of interest. However, by utilizing chemical shift tensor orientations in model compounds that resemble various phosphocholine segments, one can still obtain approximate orientations of the lipid segments with respect to the bilayer normal.

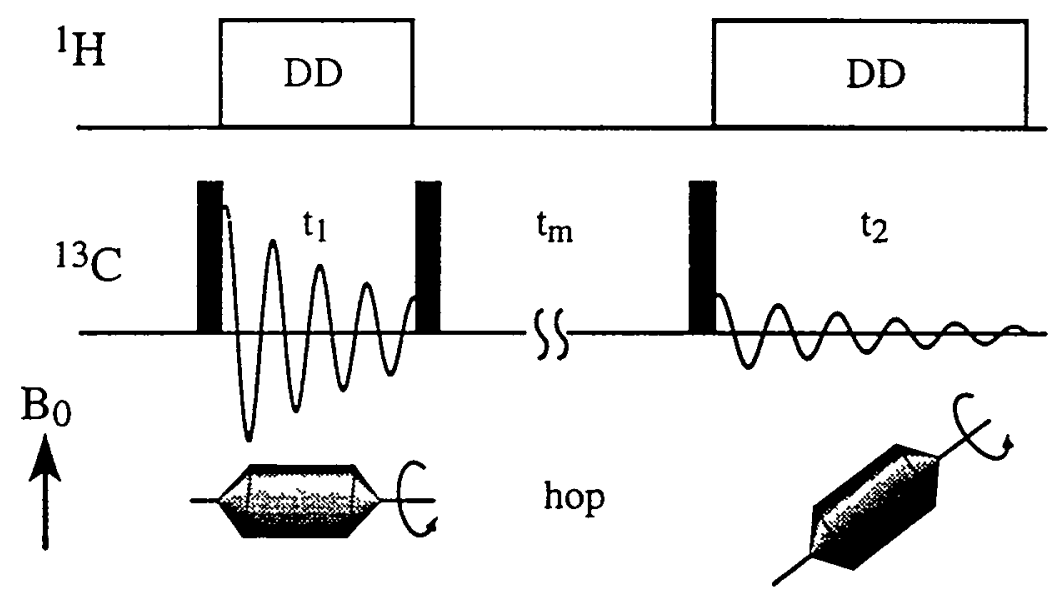

Figure 5.29 SAS pulse sequence for measuring ${ }^{13} \mathrm{C}$ chemical shift anisotropies.

The motionally-averaged ${ }^{13} \mathrm{C}$ CSAs of most sites in lecithin are obtained using the switching-angle-spinning technique. The SAS pulse sequence (Figure 5.29) involves ${ }^{13} \mathrm{C}$ chemical shift evolution under off-magic-angle spinning of the sample, followed by a mixing period to hop the rotor to the magic angle, and detection of the ${ }^{13} \mathrm{C}$ isotropic chemical shifts. Proton decoupling is employed throughout the experiment. In the resulting $2 \mathrm{D}$ spectrum, anisotropic ${ }^{13} \mathrm{C}$ chemical shift powder spectra are separated according to the isotropic ${ }^{13} \mathrm{C}$ chemical shifts of the various sites. With this pulse 
sequence, the powder spectra in $\omega_{1}$ are centered about the isotropic chemical shifts, thus the spectral intensities are distributed about the 2D diagonal. For simplicity, this 2D spectrum is sheared so that the powder patterns are centered about the zero frequency of the $\omega_{1}$ dimension.

A lecithin ${ }^{13} \mathrm{C}$ chemical shift SAS spectrum is displayed in Figure 5.30. In the aliphatic region (Figure 5.30a), the ${ }^{13} \mathrm{C} \mathrm{CSAs} \mathrm{decrease} \mathrm{from} \mathrm{the} \mathrm{segments} \mathrm{near} \mathrm{the} \mathrm{glycerol}$ to those at the chain end. The $\left(\mathrm{CH}_{2}\right)_{\mathrm{n}}$ sites exhibit the broadest powder pattern, with a width $\Delta \bar{\sigma}=\sigma_{/ /} \sigma_{\perp}$ of about $-15.2 \mathrm{ppm}$. In the isotropic dimension, the $\left(\mathrm{CH}_{2}\right)_{\mathbf{n}}$ signal spans a range of frequencies due to the superposition of many sites in the middle of the acyl chain ( $n>3$ and $n \neq \omega, \omega-1, \omega-2)$. In the acyl chain $C 2$ slice, two overlapping powder patterns with different singularities are observed. The broad component has a width of about $-17.1 \mathrm{ppm}$ and the narrow component is about $-6.6 \mathrm{ppm}$ wide. This indicates that the two phosphocholine acyl chains have different orientations near the carboxyl group. Based on ${ }^{2} \mathrm{H}$ NMR results, it has been suggested that the $s n-2$ chain first extends nearly perpendicular to the glycerol backbone and then bends at the carboxyl group, whereas the $s n-1$ chain hangs straight from the glycerol segments. Such a conformational difference is reflected both by the $\mathrm{C} 2 \mathrm{CSAs}$ measured here and by the two different $\mathrm{C} 2-\mathrm{H} 2$ dipolar couplings (Figure 5.6).

Between the ${ }^{13} \mathrm{C}$ isotropic chemical shifts of $25 \mathrm{ppm}$ and $29 \mathrm{ppm}$, the spectrum is relatively crowded due to several sites near the double bonds. Their CSAs exhibit different signs. For example, the slice at $26 \mathrm{ppm}$, assigned to the $\mathrm{C} 11$ site between two double bonds in a $\mathrm{C}^{9,12(\Delta)}$ chain, exhibits a positive CSA of $+6.6 \mathrm{ppm}$. In comparison, the $\mathrm{C} 8$ $(\sim 27.5 \mathrm{ppm})$ and $\mathrm{C} 14(\sim 26.5 \mathrm{ppm})$ sites one bond away from the double bonds exhibit small negative CSAs. The positive CSA of C11 may be attributed to the steric constraint at this segment: the two flanking double bonds are likely to make the orientation and the electronic environment of the $\mathrm{C} 11$ site different from the rest of the acyl segments. 
(a)

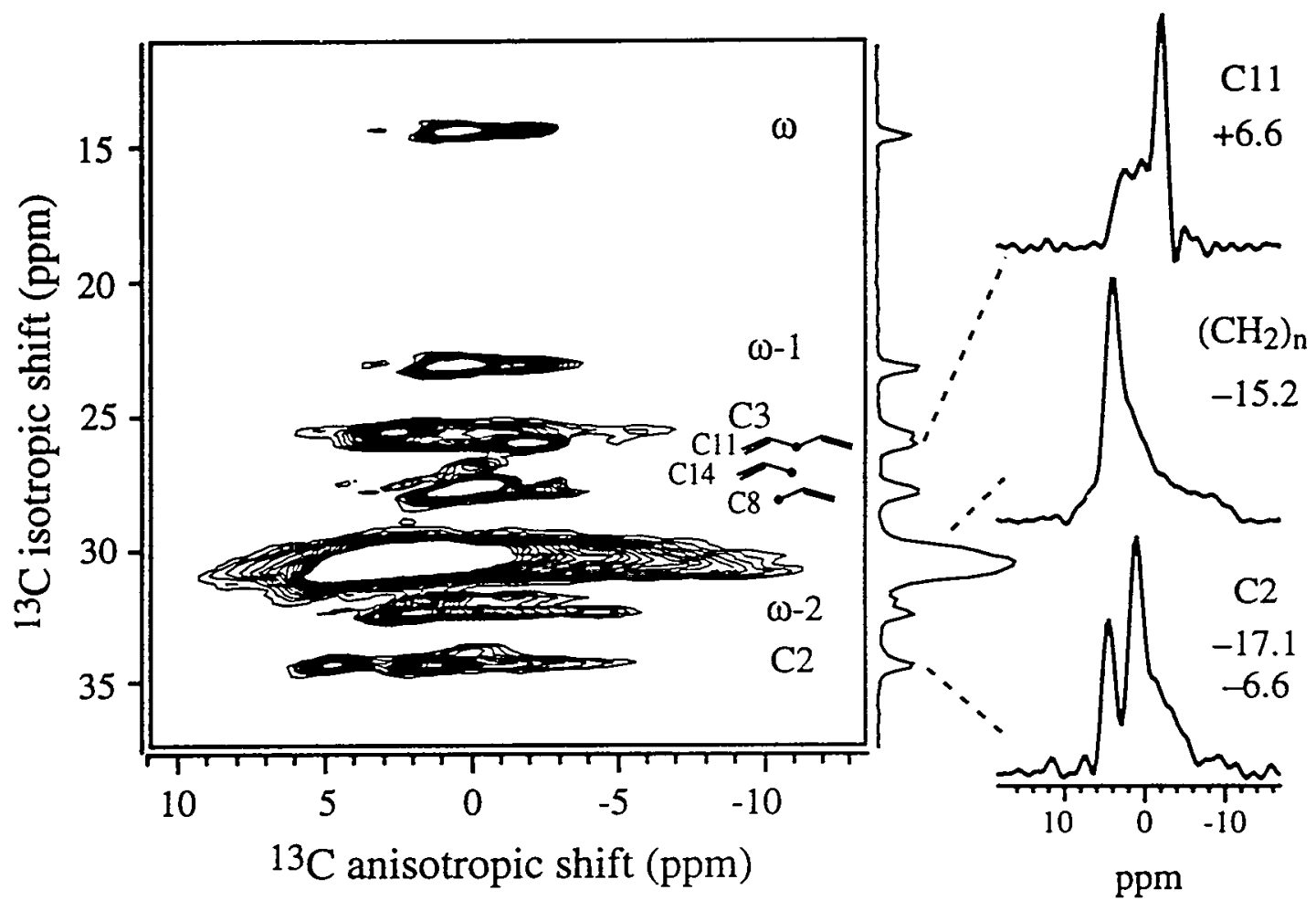

Figure 5.30 $\mathrm{L}_{\alpha}$-lecithin 2D ${ }^{13} \mathrm{C}$ SAS spectrum. (a) Acyl chain region. (b) Headgroup and glycerol regions. (c) Double bond region. 


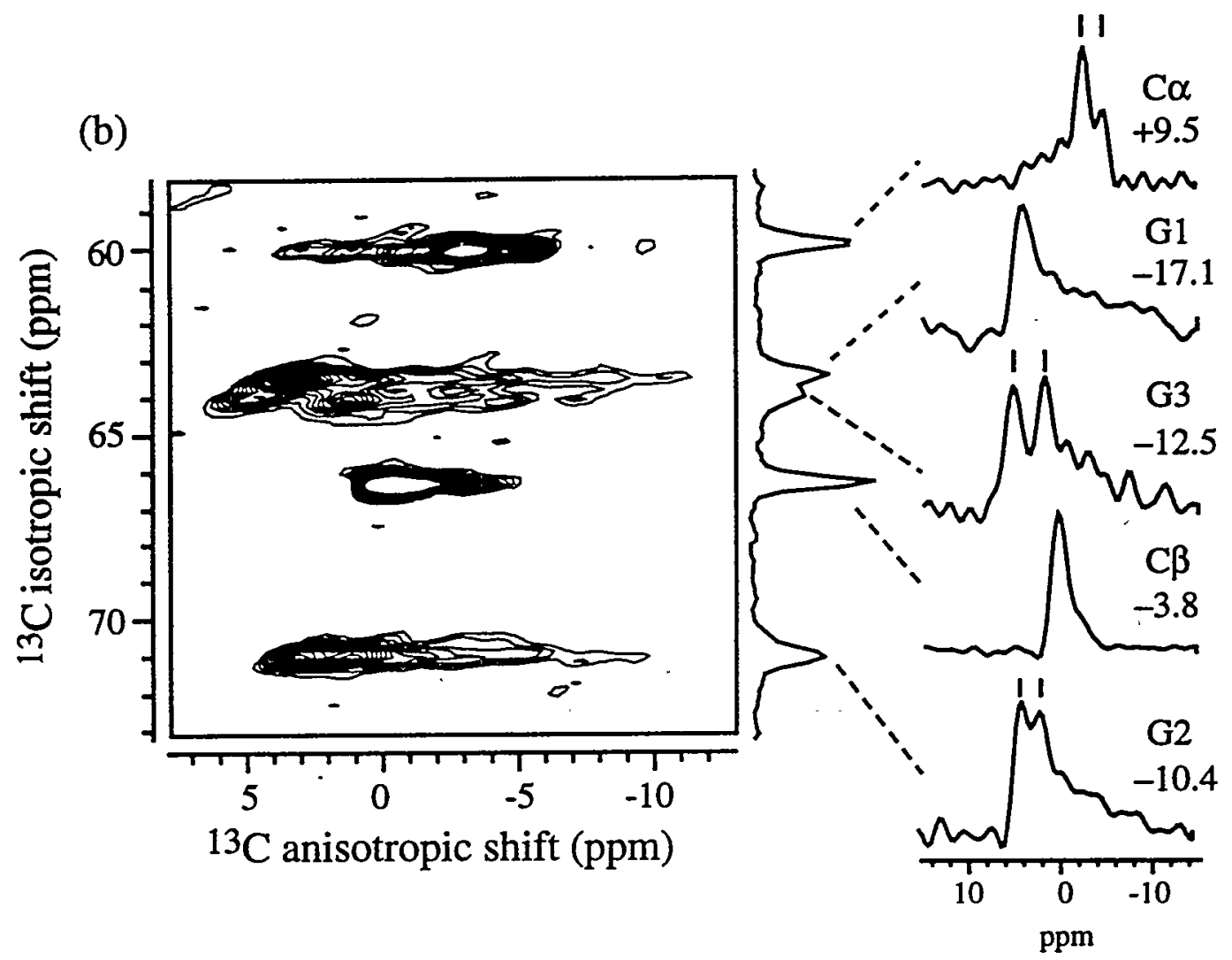

(c)

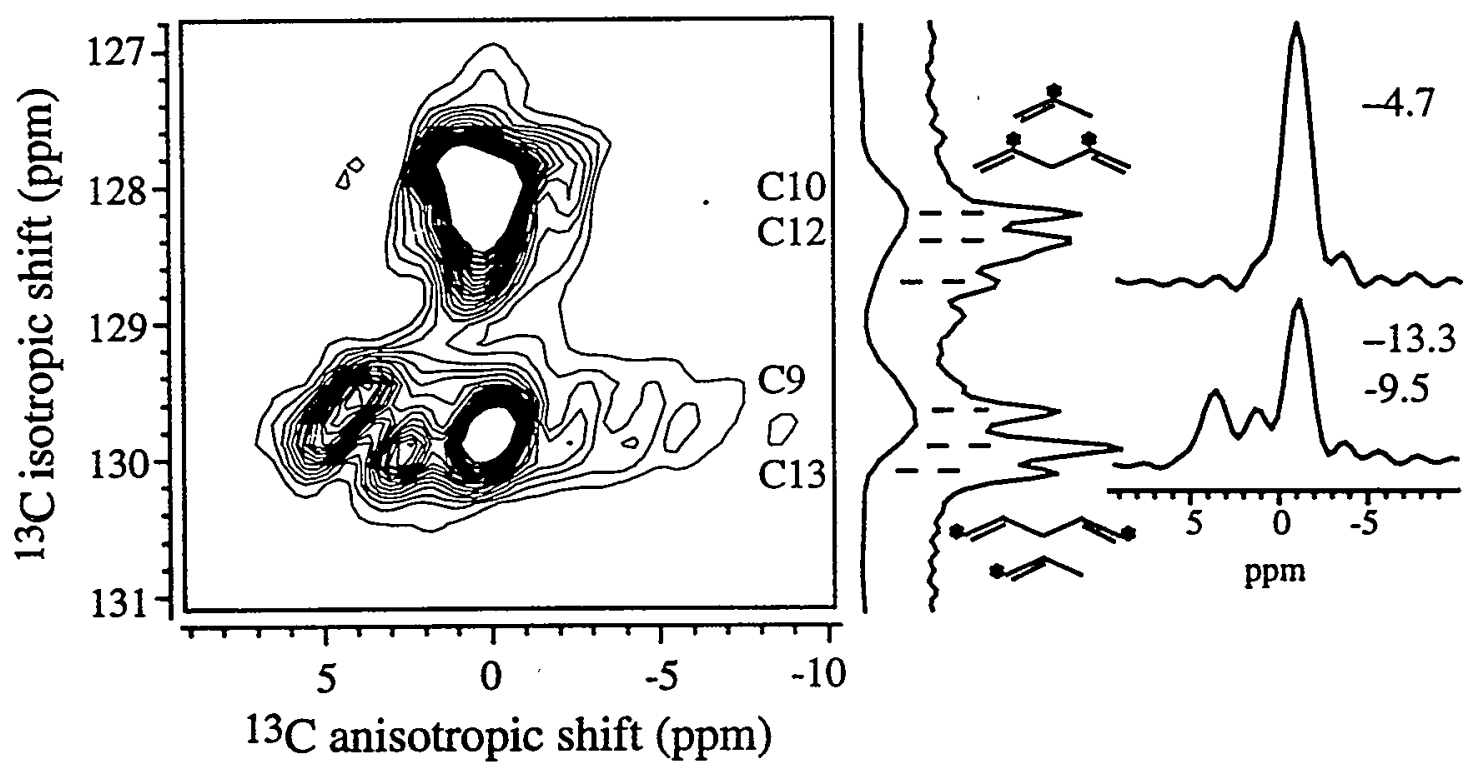


The ${ }^{13} \mathrm{C}$ CSAs of the glycerol and headgroup segments are shown in Figure $5.30 \mathrm{~b}$. Splittings of about $200 \mathrm{~Hz}$ can be clearly seen in the chemical shift powder patterns of G3 $(264 \mathrm{~Hz}), \mathrm{G} 2(144 \mathrm{~Hz})$ and $\mathrm{C} \alpha(168 \mathrm{~Hz})$ sites. These splittings result from the P-C dipolar couplings, since the ${ }^{31} \mathrm{P}$ nucleus is relatively close to these headgroup and glycerol carbons. The C $\alpha$ powder pattern has an opposite sign from the others, which can be attributed to the fact that $\mathrm{C} \alpha$ is situated at the bending point of the headgroup from the glycerol backbone. Also, large negative CSAs (yielding positive narrowing factors) are observed for the glycerol sites, suggesting that the motional axis is preferentially parallel to the glycerol backbone.

Figure 5.30c shows the chemical shift spectra of the double bond carbons between $127 \mathrm{ppm}$ and $131 \mathrm{ppm}$ isotropic shifts. Four carbon sites, $\mathrm{C} 10, \mathrm{C} 12, \mathrm{C} 9$ and $\mathrm{C} 13$, can be

resolved. ${ }^{8}$ However, the anisotropic widths of these chemical shift patterns are only qualitative from the spectrum.

To put these ${ }^{13} \mathrm{C}$ chemical shift anisotropies into perspective with the other NMR anisotropies (mainly dipolar couplings) in $\mathrm{L}_{\alpha}$-phosphocholine, we can calculate the order parameters of these segmental CSAs, using the chemical shift tensor values determined in model compounds as the rigid-iattice anisotropies. The resulting CSA order parameters and the measured averaged CSAs are listed in Table 5.1 and plotted in Figure 5.35. Before we present these NMR order parameters, we first complete the description of our NMR measurements of phosphocholine with the ${ }^{31} \mathrm{P}$ and ${ }^{1} \mathrm{H}$ chemical shift anisotropies.

\section{$5.10{ }^{31} \mathrm{P}$ and ${ }^{1} \mathrm{H}$ Chemical Shifts}

The ${ }^{31} \mathrm{P}$ chemical shift serves as a sensitive indicator of the phospholipid phase: the $\mathrm{L}_{\alpha}$ phase has a distinct $\eta=0$ lineshape and a specific averaged anisotropy. For phosphocholine lipids, the averaged anisotropy $\Delta \bar{\sigma}$ is about $-47 \mathrm{ppm}$ (Figure 4.1). 
We observe temperature-dependent ${ }^{31} \mathrm{P}$ chemical shift anisotropies in $\mathrm{PE}$ and the PC-cholesterol mixture. Although we did not study this lineshape change further, we can hypothesize that mixed phases (e.g. $\mathrm{L}_{\alpha}$ versus $\mathrm{H}_{\text {II }}$ phase) are induced by the temperature variation and by the addition of cholesterol in phosphocholine.

We have shown the ${ }^{1} \mathrm{H}$ isotropic chemical shifts in lecithin in a $1 \mathrm{D}$ MAS spectrum (Figure 5.9d). The anisotropic ${ }^{1} \mathrm{H}$ chemical shifts are ruovre difficult to obtain due to the large $\mathrm{H}-\mathrm{H}$ homonuclear couplings. Nevertheless, we obtained partial information on ${ }^{1} \mathrm{H}$ CSAs by correlating ${ }^{31} \mathrm{P}$ and ${ }^{1} \mathrm{H}$ CSAs using the pulse sequence in Figure 5.31. In the evolution period $t_{1},{ }^{1} \mathrm{H}$ magnetization evolves under the chemical shift interaction, with $\mathrm{H}-$ $\mathrm{H}$ and $\mathrm{P}-\mathrm{H}$ dipolar couplings suppressed by a MREV-8 multiple-pulse train and a $180^{\circ} 31 \mathrm{P}$ pulse in the middle of the $t_{1}$ period, respectively. At the end of $t_{1}$, a ${ }^{1} \mathrm{H} z$-filter records the cosine component of the magnetization, which is then transferred to ${ }^{31} \mathrm{P}$ by $\mathrm{CP}$. Subsequently, a $z$-filter on ${ }^{31} \mathrm{P}$ is applied to amplitude-modulate the ${ }^{31} \mathrm{P}$ magnetization, which is detected in the $t_{2}$ period with ${ }^{1} \mathrm{H}$ decoupling. The experiment is conducted under the static condition, which gives rise to unscaled chemical shift spectra.

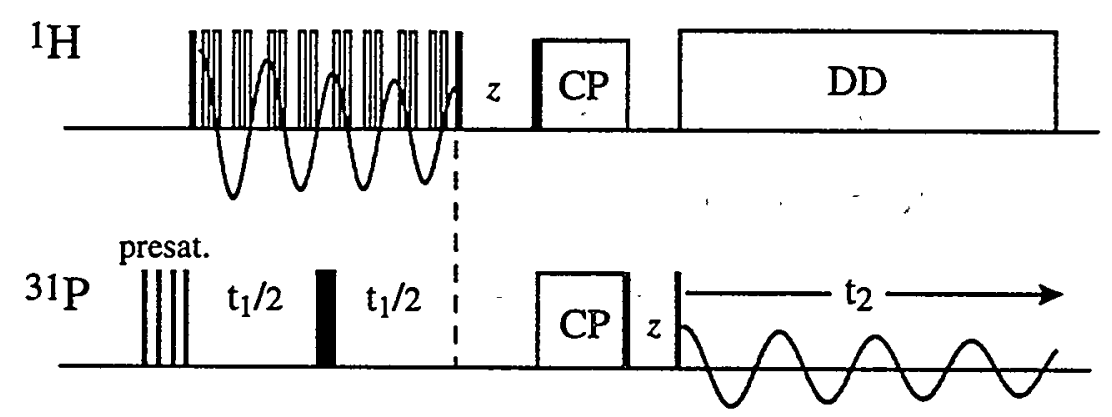

Figure $5.31{ }^{31} \mathrm{P} /{ }^{1} \mathrm{H}$ shift correlation pulse sequence.

A $2 \mathrm{D}{ }^{31} \mathrm{P} /{ }^{1} \mathrm{H}$ shift correlation spectrum of lecithin, together with the projection of the ${ }^{31} \mathrm{P}$ chemical shift, is presented in Figure 5.32. At the isotropic ${ }^{31} \mathrm{P}$ chemical shift, a 


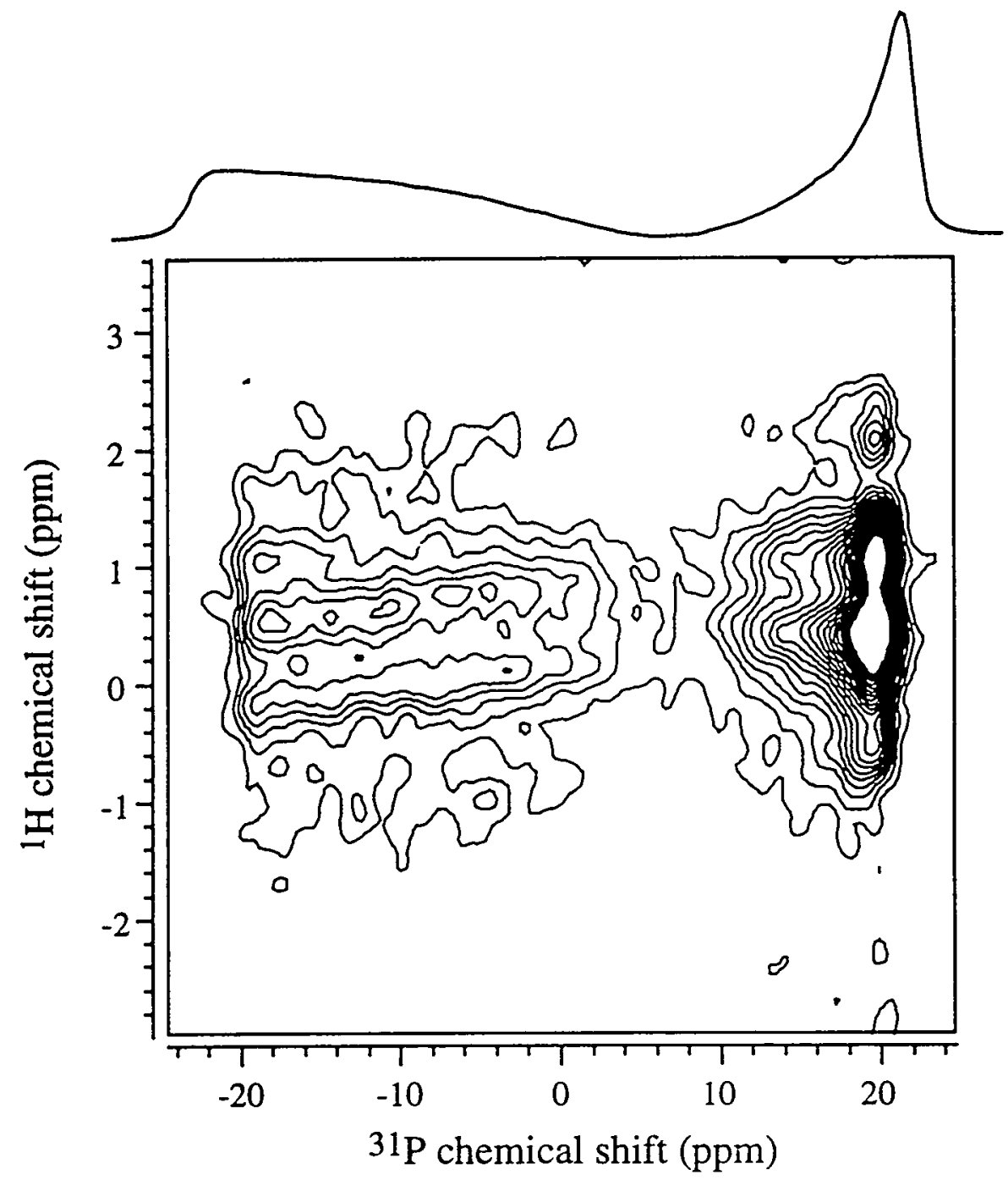

Figure 5.32 Lecithin ${ }^{31} \mathrm{P} /{ }^{1} \mathrm{H}$ CSA correlation 2D spectrum. 
magic-angle hole is observed, thus the corresponding isotropic ${ }^{1} \mathrm{H}$ chemical shift cross section has vanishing intensities. The anisotropic ${ }^{1} \mathrm{H}$ chemical shifts can be best extracted at the $90^{\circ}$ edge of the ${ }^{31} \mathrm{P}$ powder pattern, since $\mathrm{S} / \mathrm{N}$ is the highest in this slice. The ${ }^{1} \mathrm{H}$ chemical shift spectrum of lecithin scaled by $P_{2}\left(\cos 90^{\circ}\right)=-0.5$ is displayed in Figure 5.33, which also contains the ${ }^{1} \mathrm{H}$ cross sections of PE, SPM, and PC with cholesterol. It can be seen that the ${ }^{1} \mathrm{H}$ resonances overlap due to their anisotropies, even though orientational broadening has been intrinsically removed by correlation of the two interactions in a $2 \mathrm{D}$ fashion. Extrapolating from the potential isotropic ${ }^{1} \mathrm{H}$ chemical shifts in the magic-angle slice, and taking into account the ${ }^{1} \mathrm{H}$ intensity distribution in the $0^{\circ}$ cross section of the ${ }^{31} \mathrm{P}$ powder pattern, the ${ }^{1} \mathrm{H}$ spectrum at the $90^{\circ}$ edge can be assigned tentatively, as demonstrated for lecithin (Figure 5.33a). The assignment is based on two requirements.

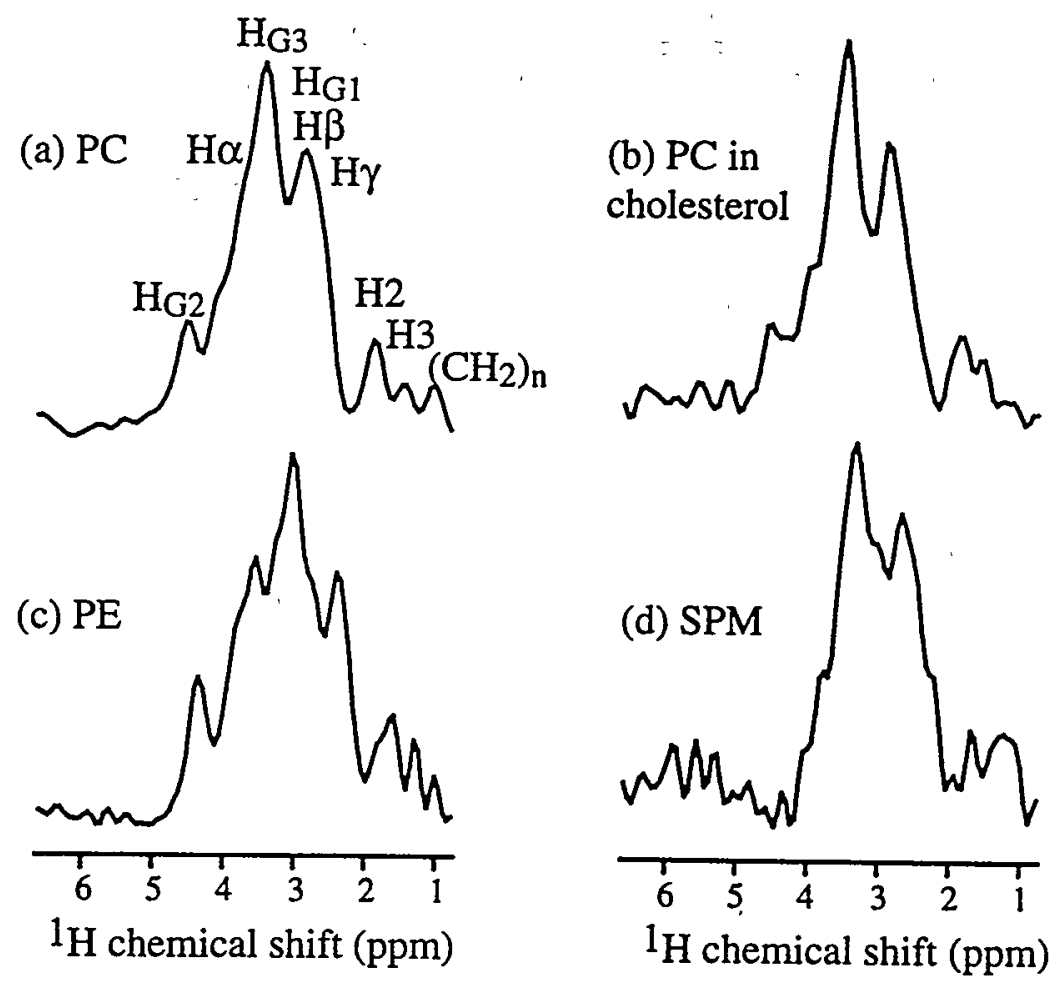

Figure $5.33{ }^{1} \mathrm{H}$ chemical shift cross sections correlated with the $90^{\circ}$ edge of the ${ }^{31} \mathrm{P}$ powder patterns. (a) PC. (b) PC with cholesterol. (c) PE. (d) SPM. 


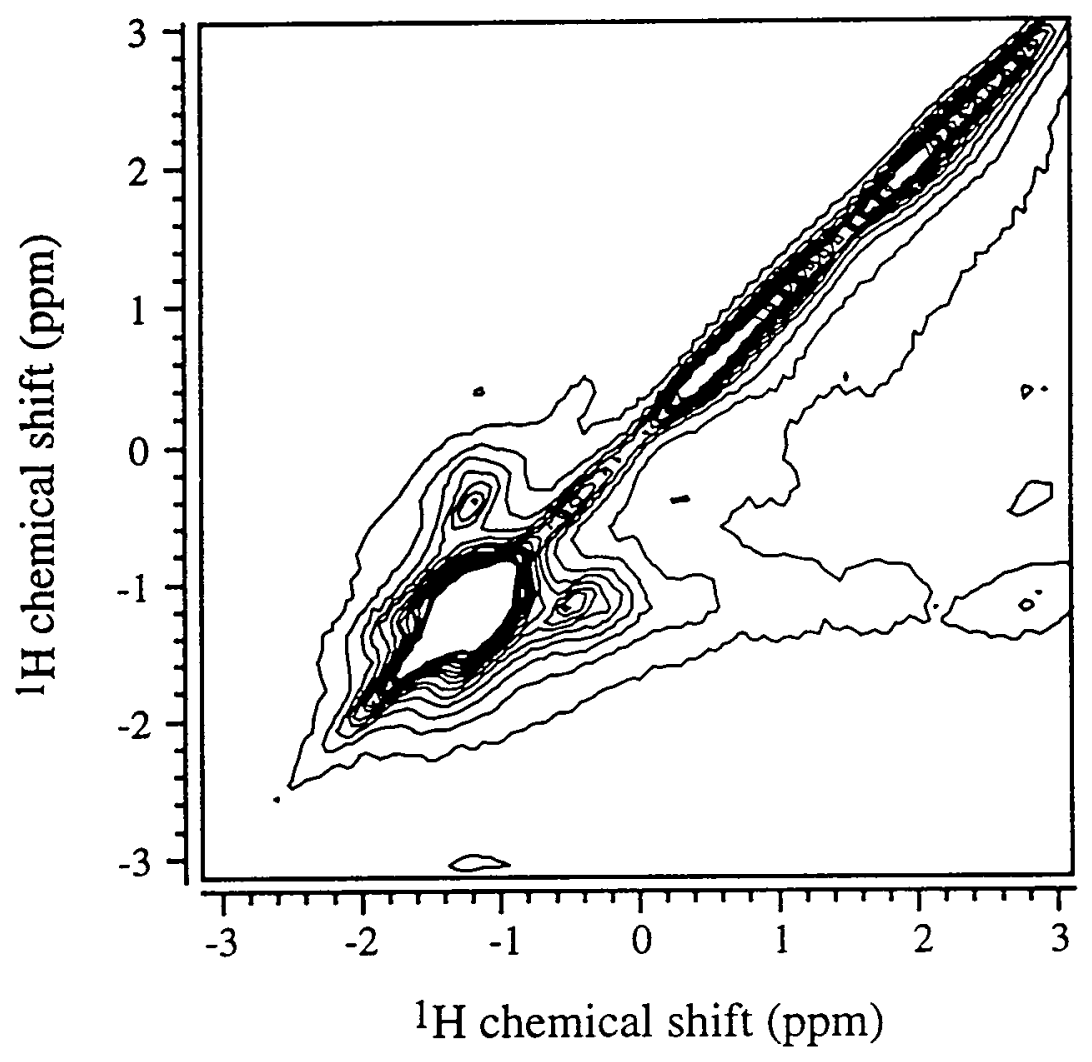

Figure 5.34 $\mathrm{L}_{\alpha}$-lecithin $2 \mathrm{D}{ }^{1} \mathrm{H}$ exchange spectrum. The experiment was performed under the static condition, with an exchange mixing time of $10 \mathrm{~ms}$. Little exchange is observed for the lipid ${ }^{1} \mathrm{H}$ sites. The frequency scales are only approximate. 
First, the ${ }^{1} \mathrm{H}$ CSAs in the $0^{\circ}$ slice must be twice of those in the $90^{\circ}$ slice. Second, for each inequivalent proton, the chemical shift pattern must converge to a single frequency in the magic angle slice. Unfortunately, due to the small ${ }^{1} \mathrm{H}$ chemical shift dispersion and the low resolution of the static spectrum relative to a MAS spectrum, quantitative ${ }^{1} \mathrm{H} C S A s$ can not be extracted, and can only be approximated by simulations.

It is interesting that the ${ }^{1} \mathrm{H}$ cross section for pure $\mathrm{PC}$ is similar to that for $\mathrm{PC}$ with cholesterol. Yet the PE and SPM spectra differ significantly from the PC spectrum. More experiments would need to be performed to obtain the exact ${ }^{1} \mathrm{H}$ CSAs and to incorporate them into structural analysis of the phospholipids.

We also carried out a static ${ }^{1} \mathrm{H}$ chemical shift $2 \mathrm{D}$ exchange experiment on phosphocholine. The spectrum, obtained with a mixing time of $10 \mathrm{~ms}$, is shown in Figure 5.34. As seen, spectral intensities are mainly localized along the diagonal of the 2D spectrum, which indicates that little spin diffusion or chemical exchange occurs within this mixing time. Only the broad water peak exhibits non-diagonal intensities. Since the ${ }^{1} \mathrm{H}$ CSAs are unscaled, site overlap is severe in the spectrum.

\subsection{Summary of NMR Order Parameters in Lecithin}

The many NMR chemical shift anisotropies $\left({ }^{13} \mathrm{C},{ }^{31} \mathrm{P},{ }^{1} \mathrm{H}\right)$ and dipolar couplings (C-H, P-H, H-H, C-C) we measured in phosphocholine can be put into perspective by evaluating and comparing their corresponding order parameters. Several averaged dipolar couplings, including the $\mathrm{P}-\mathrm{C} \alpha, \mathrm{P}-\mathrm{G} 3, \mathrm{CO} 2-\mathrm{C} 2, \mathrm{H} \alpha-\mathrm{H} \alpha$ and $\mathrm{H}_{\mathrm{G} 3}-\mathrm{H}_{\mathrm{G} 3}$ couplings, occur between nuclei separated by one or two bonds and thus have fixed internuclear distances. This means that the rigid-limit coupling constants of these dipolar pairs can be calculated, and the order parameters of these internuclear vectors can be determined as the ratio between the averaged and the rigid-limit couplings. The calculation is similar to that for the $\mathrm{C}-\mathrm{H}$ bond order parameters (Figure 5.6). However, except for the $\mathrm{CO} 2-\mathrm{C} 2$ coupling, the 
signs of these non-C-H dipolar couplings are undetermined from the experiments conducted.

To obtain the chemical shift order parameters, the rigid-lattice chemical shift tensor is required. The chemical shift principal-axis orientations and values for various sites in phosphocholine can be approximated by the tensor values determined in model compounds. For example, the ${ }^{13} \mathrm{C}$ chemical shift tensors of the glycerol segments can be well approximated by the tensors in the model compound methyl- $\alpha$-D-glucopyranoside (MGP). ${ }^{34}$ This sugar derivative contains a segment $\mathrm{HO}-\mathrm{CH}_{2}-\mathrm{CH}(\mathrm{OC} .)-.\mathrm{CH}(\mathrm{OH})-\mathrm{C}$, whose $\mathrm{CH}_{2}$ and $\mathrm{CH}$ sites exhibit isotropic chemical shifts very similar to the $\mathrm{G} 3 / \mathrm{Gl}$ and G2 sites in phosphocholine. As another example, the chemical shift tensor of the $C \alpha$ carbon can be approximated by an average of the tensors of the methylenes in MGP and in poly(ethylene terephthalate) (PET). The methylene carbons in these molecules also exhibit isotropic chemical shifts similar to that of $\mathrm{C} \alpha .{ }^{35}$ Since the ${ }^{13} \mathrm{C}$ SAS spectra and the $1 \mathrm{D}{ }^{31} \mathrm{P}$ spectrum yield the signs of the averaged CSAs, the signs of the ${ }^{13} \mathrm{C}$ and ${ }^{31} \mathrm{P}$ chemical shift order parameters $\bar{\delta} / \delta$ can be determined. These order parameters provide information on the averaged orientation of the main principal axis, i.e. the axis with the largest principal value, of the chemical shift tensor with respect to the director.

Table 5.1 summarizes the ${ }^{13} \mathrm{C}$ chemical shift order parameters for all resolved carbon sites in $L_{\alpha}$-phosphocholine. The experimental isotropic ${ }^{13} \mathrm{C}$ chemical shifts are listed and compared with the isotropic shifts of the model compounds. The difference between the two isotropic shifts for each carbon serves as a gauge of the suitability of the model compound to approximate the rigid-lattice chemical shift tensor of the phosphocholine segment. Two types of rigid-lattice CSAs, $\Delta \sigma=\sigma_{z z}-\sigma_{x x}$ and $\delta=\sigma_{z z}$ $\sigma_{i s o}$, are tabulated. Due to the lack of perfect axial symmetry for most chemical shift tensors $(\eta \neq 0)$, these CSAs give rise to two different definitions of the chemical shift order parameters, $\bar{\delta} / \delta$ and $\Delta \bar{\sigma} / \Delta \sigma$. The two order parameters are identical when the tensor has axial symmetry $(\eta=0)$ and deviate the most, by a factor of 0.75 , when the tensor 


\begin{tabular}{|c|c|c|c|c|c|}
\hline site & $\begin{array}{l}\sigma_{\text {iso }}(\mathrm{ppm}) \\
\text { exp./ model }\end{array}$ & $\begin{array}{c}\Delta \bar{\sigma}=\frac{3}{2} \bar{\delta} \\
(\mathrm{ppm})\end{array}$ & $\begin{array}{c}\Delta \sigma(\text { model }) \\
(\mathrm{ppm})\end{array}$ & $\begin{array}{c}\delta \text { (model) } \\
(\mathrm{ppm})\end{array}$ & $\bar{\delta} / \delta$ \\
\hline$C \gamma$ & $54.4 / 56$ & $+3.0 \pm 1.0$ & -75 (MGP) $^{34}$ & -45.0 & -0.044 \\
\hline$C \beta$ & $66.3 / 61.7$ & $-3.8 \pm 0.5$ & $-65(\mathrm{PET})^{35}$ & -40.0 & +0.063 \\
\hline $\mathrm{C} \alpha$ & $59.8 / 61.7$ & $+10.5 \pm 1.0$ & $-65(\mathrm{PET})^{35}$ & -40.0 & -0.175 \\
\hline G3 & $63.9 / 63.3$ & $-12.5 \pm 2.0$ & $-56(\mathrm{MGP})^{34}$ & -30.3 & +0.275 \\
\hline G2 & $71.2 / 72.0$ & $-10.4 \pm 1.5$ & $-33(\mathrm{MGP})^{34}$ & -19.0 & +0.365 \\
\hline G1 & $63.4 / 63.3$ & $-16.0 \pm 2.0$ & $-56(\mathrm{MGP})^{34}$ & -30.3 & +0.352 \\
\hline $\mathrm{CO} 1$ & $173.3 / 173.0$ & $+27.5 \pm 3.0$ & $-143(\mathrm{DPPE})^{36}$ & +87.7 & +0.209 \\
\hline $\mathrm{CO} 2$ & $173.3 / 173.0$ & $-1.9 \pm 1.0$ & $-143(\mathrm{DPPE})^{36}$ & +87.7 & -0.014 \\
\hline $\mathrm{C} 2^{\prime}$ & $34.4 / 43.3$ & $-17.0 \pm 2.0$ & $-44(\mathrm{mal})^{37}$ & -25.3 & +0.448 \\
\hline $\mathrm{C} 2$ & $34.4 / 43.3$ & $-6.6 \pm 1.0$ & $-44(\mathrm{mal})^{37}$ & -25.3 & +0.174 \\
\hline C3 & $25.9 / 33.0$ & $-9.5 \pm 1.0$ & $-37(\mathrm{PE})^{35}$ & -20.5 & +0.309 \\
\hline$\left(\mathrm{CH}_{2}\right)_{n}$ & $31.6 / 33.0$ & $-15.2 \pm 2.0$ & $-37(\mathrm{PE})^{35}$ & -20.5 & +0.494 \\
\hline $\mathrm{C} 8$ & $27.7 / 28.0$ & $-4.7 \pm 1.0$ & $-34(\mathrm{muc})^{37}$ & -22.0 & +0.142 \\
\hline $\mathrm{C} 9,13$ & $130.2 / 124.3$ & $-13.3 \pm 1.5$ & $+210(\mathrm{bte}, \mathrm{cis})^{37}$ & +107.7 & -0.082 \\
\hline $\mathrm{C} 11$ & $\sim 26.0 / 28.0$ & $+6.6 \pm 1.0$ & $-34(\mathrm{muc})^{37}$ & -22.0 & -0.200 \\
\hline $\mathrm{C} 10,12$ & $128.2 / 124.3$ & $-4.7 \pm 1.0$ & $+210\left(\right.$ bte, cis) ${ }^{37}$ & +107.7 & -0.029 \\
\hline $\mathrm{C} 14$ & $\sim 26.5 / 28.0$ & $<1.5 \pm 0.5$ & $-34(\mathrm{muc})^{37}$ & -22.0 & -0.045 \\
\hline$\omega-2$ & $32.5 / 35.0$ & $-8.5 \pm 1.5$ & $-33(\mathrm{eic})^{37}$ & 18.0 & +0.315 \\
\hline$\omega-1$ & $23.0 / 26.3$ & $-4.0 \pm 1.0$ & $-22(\mathrm{eic})^{37}$ & -12.0 & +0.217 \\
\hline$\omega$ & $14.2 / 16.3$ & $-3.8 \pm 1.0$ & $-24(\text { eic })^{37}$ & -14.0 & +0.177 \\
\hline
\end{tabular}

Table 5.1 NMR ${ }^{13} \mathrm{C}$ chemical shift order parameters in $L_{\alpha}$-lecithin. MGP: methyl- $\alpha$ D-glucopyranoside; PET: poly(ethylene terephthalate); DPPE: dipalmitoylphosphatidylethanol-amine. mal: malonic acid; PE: polyethylene; muc: dihydromuconic acid; bte: cis-2-butene; eic: n-eicosane; 
asymmetry is $\eta=1$. Specifically, $\bar{\delta} / \delta$ indicates the motional narrowing factor along a well-defined direction fixed in the segment, i.e. the $\sigma_{z z}$ axis of the chemical shift PAS, whereas $\Delta \bar{\sigma} / \Delta \sigma$ is only a general indicator of the degree of motional averaging of the whole segment. We choose the $\bar{\delta} / \delta$ order parameter to characterize the motionallyaveraged orientations of the phosphocholine segments.

Table 5.1 shows that most ${ }^{13} \mathrm{C}$ sites in phosphocholine have positive CSA order parameters except for the headgroup and double-bond segments. The positive sign of these order parameters can be explained by the orientation of the $\sigma_{z z}$ principal axis in most methylene groups: it is normal to the $\mathrm{H}-\mathrm{C}-\mathrm{H}$ plane. A positive $\bar{\delta} / \delta$ indicates that the $\sigma_{z z}$ axis lies preferentially parallel to the molecular rotation axis, or that the $\mathrm{H}-\mathrm{C}-\mathrm{H}$ plane is preferentially perpendicular to it. This is consistent with the negative $\mathrm{C}-\mathrm{H}$ bond order parameters of most acyl chain and glycerol segments (Figure 5.6), since they indicate that the $\mathrm{H}-\mathrm{C}-\mathrm{H}$ planes are nearly perpendicular to the bilayer normal. Therefore, the ${ }^{13} \mathrm{C}$ chemical shift $\left(\sigma_{z z}\right)$ and the $\mathrm{C}-\mathrm{H}$ order parameters give consistent results on the segmental orientations in phosphocholine.

\begin{tabular}{c|ccc}
\hline couplings & $\bar{\delta}(\mathrm{kHz})$ & $\delta(\mathrm{kHz})$ & $\bar{\delta} / \delta$ \\
\hline $\mathrm{H} \alpha-\mathrm{H} \alpha$ & $\pm 1.9 \pm 0.3$ & -31.4 & \pm 0.061 \\
$\mathrm{P}-\mathrm{C} \alpha$ & $\pm 0.160 \pm 0.015$ & -0.660 & \pm 0.242 \\
${ }^{31} \mathrm{PO}_{4}$ & $-31.3 \pm 1.5 \mathrm{ppm}$ & $+108.7 \mathrm{ppm}^{38}$ & -0.288 \\
$\mathrm{P}-\mathrm{G} 3$ & $\pm 0.260 \pm 0.020$ & -0.660 & \pm 0.394 \\
$\mathrm{H}_{\mathrm{G} 3}-\mathrm{H}_{\mathrm{G} 3}$ & $\pm 6.8 \pm 0.3$ & -31.4 & \pm 0.216 \\
$\mathrm{CO}_{2}-\mathrm{C} 2$ & $-0.040 \pm 0.022$ & -2.08 & +0.0192 \\
\hline
\end{tabular}

Table 5.2 Several NMR dipolar order parameters and ${ }^{31} \mathrm{P}$ chemical shift order parameter in phosphocholine. 
Table 5.2 summarizes the order parameters of the five dipolar couplings mentioned above and the ${ }^{31} \mathrm{P}$ chemical shift order parameter in phosphocholine. The $\mathrm{CO} 2-\mathrm{C} 2$ order parameter is determined to be positive from the SAS spectra (Figures 5.21 and 5.22), and the ${ }^{31} \mathrm{P}$ chemical shift spectrum (Figure 4.1) indicates a negative order parameter. Other order parameters have undetermined signs. However, judging from the headgroup bend in the molecule, which is corroborated, for instance, by the sign inversion of the $\mathrm{C} \alpha-\mathrm{H} \alpha$ order parameter from the glycerol ones, we postulate that the $\mathrm{P}-\mathrm{C} \alpha$ order parameter is negative while the $\mathrm{P}-\mathrm{G} 3$ order parameter is positive.

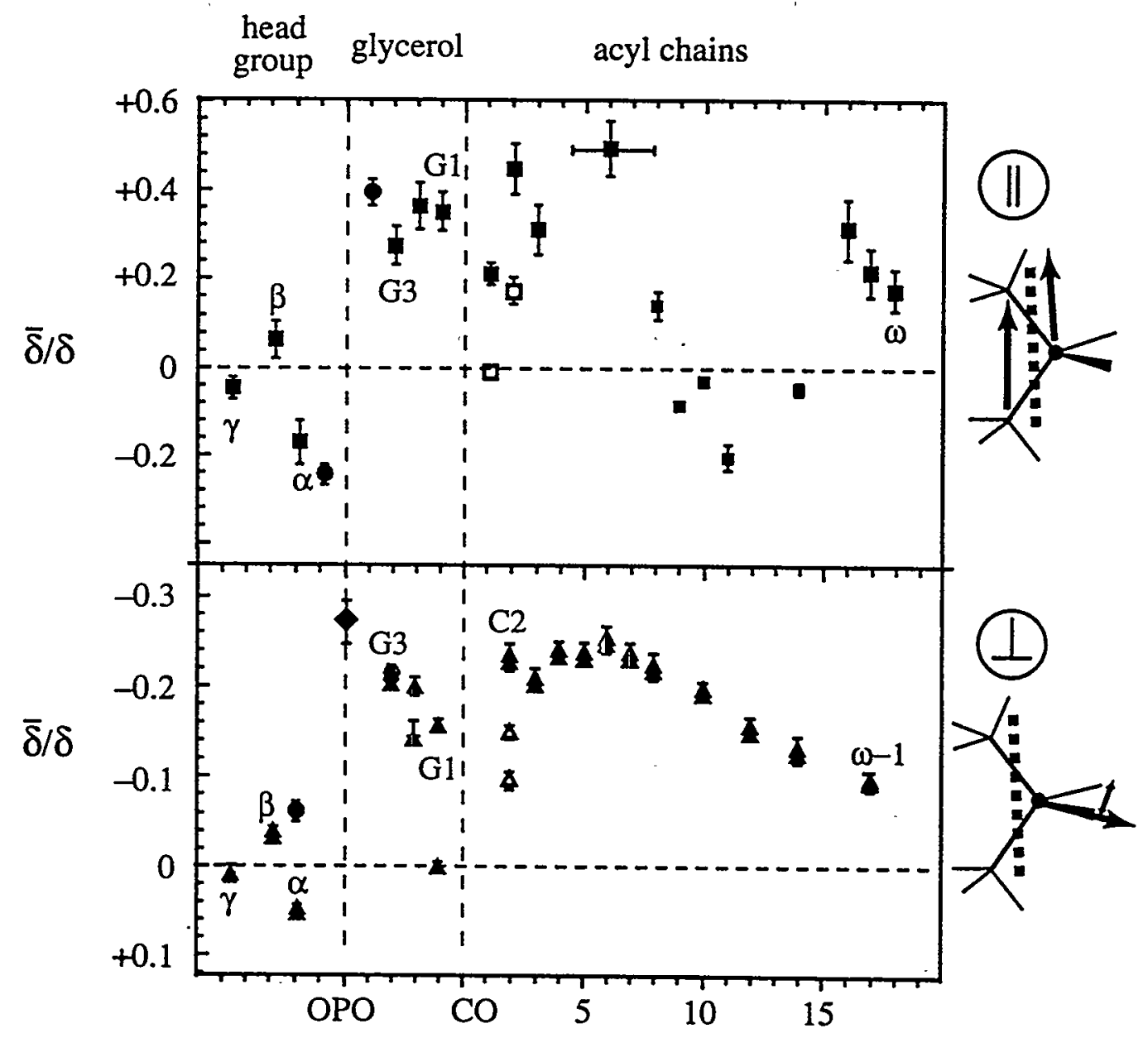

Figure 5.35 NMR order parameters in $\mathrm{L}_{\alpha}$-phosphocholine. The symbols represent: a : ${ }^{13} \mathrm{C} \mathrm{CSA} ;:^{31} \mathrm{P} \mathrm{CSA} \wedge: \mathrm{C}-\mathrm{H} ; \bullet: \mathrm{P}-\mathrm{C}$ and $\mathrm{H}-\mathrm{H}$. 
The NMR order parameters listed in the above two tables, combined with the $\mathrm{C}-\mathrm{H}$ bond order parameters shown before, are plotted in Figure 5.35 as a summary of our experimental short-range NMR couplings in $\mathrm{L}_{\alpha}$-phosphocholine. The mostly positive ${ }^{13} \mathrm{C}$ CSA and P-C order parameters are shown in the top of the figure, while the predominantly negative $\mathrm{C}-\mathrm{H},{ }^{31} \mathrm{~F}$ and $\mathrm{H}-\mathrm{H}$ order parameters are shown in the bottom. The division is based on the different orientations of the $z$ principal axis of the respective NMR interactions relative to the local backbone. As indicated schematically, the $z$ axes of the interactions in the top part of the figure are aligned parallel to the local molecular backbone, while those in the bottom are perpendicular to it. The unfilled symbols represent the $s n-2$ chain order parameters that differ significantly from the corresponding order parameters in the $s n-1$ chain. The order parameters with ambiguous signs are shown with a sign consistent with either the phosphocholine structure proposed later (chapter 6) or the structure commonly

accepted in the literature. Due to the higher precision of the ${ }^{2} \mathrm{H}$ quadrupolar couplings compared to the $\mathrm{C}-\mathrm{H}$ dipolar couplings obtained from our PDLF spectra, the $\mathrm{C}-\mathrm{H}$ order parameters are plotted with ${ }^{2} \mathrm{H}$-derived magnitudes and dipolar-derived signs.

\section{References}

(1) Seelig, J.; Seelig, A. Q. Rev. Biophys. 1980, 13, 19.

(2) Scherer, P. G.; Seelig, J. Biochemistry 1989, 28, 7723.

(3) Salinger, Z.; Lapidot, Y. J. Lipid Res. 1966, 7, 174.

(4) Radhakrishnan, R.; Robson, R. J.; Takogaki, Y.; Khorana, H. G. Methods Enzymol. 1981, 72, 408.

(5) Hermetter, A.; Paltauf, F. Chem. Phys. Lipids 1981, 28, 111.

(6) Eastman, M. A.; Grandinetti, P. J.; Lee, Y. K.; Pines, A. J. Magn. Reson 1992, $98,333$. 
(7) Bechinger, B.; Seelig, J. Chem. Phys. Lipids 1991, 58, 1.

(8) Husted, C.; Montez, B.; Le, C.; Moscarello, M. A.; Oldfield, E. Magn. Res. Med $1993,29,168$.

(9) Seelig, J.; Waespe-Sarcevic, N. Biochemistry 1978, 17, 3310.

(10) Skarjune, R.; Oldfield, E. Biochemistry 1979, 18, 5903.

(11) Sanders, C. R.; Prestegard, J. H. J. Am. Chem. Soc. 1991, 113, 1987.

(12) Gally, H.; Niederberger, W.; Seelig, J. Biochemistry 1975, 14, 3647.

(13) Tamm, L. K.; Seelig, J. Biochemistry 1983, 22, 1474.

(14) Strenk, L. M.; Westerman, P. W.; Doane, J. W. Biophys. J. 1985, 48, 765.

(15) Seelig, J.; Macdonald, P. M.; Scherer, P. G. Biochemistry 1987, 26, 7535.

(16) Ernst, R. R.; Bodenhausen, G.; Wokaun, A. Principles of Nuclear Magnetic Resonance in One and Two Dimensions; Clarendon Press, Oxford, 1987.

(17) Lee, C. W. B.; Griffin, R. G. Biophys. J. 1989, 55, 355.

(18) Hong, M.; Schmidt-Rohr, K.; Nanz, D. Biophys. J. 1995a, 69, 1939.

(19) Forbes, J.; Husted, C.; Oldfield, E. J. Am. Chem. Soc. 1988, 110, 1059.

(20) Hemminga, M. A.; Cullis, P. R. J. Magn. Reson. 1982, 47, 307.

(21) Schmidt-Rohr, K.; Clauss, J.; Spiess, H. W. Macromolecules 1992, 25, 3273.

(22) Bloom, M.; Burnell, E. E.; Roeder, S. B. W.; Valic, M. I. J. Chem. Phys. 1977, $66,3012$.

(23) Sanders, C. R. Biophys. J. 1993, 64, 171.

(24) Sanders, C. R.; Schwonek, J. P. Biochemistry 1992, 31, 8898.

(25) Waugh, J. S. Proc. Natl. Acad. Sci. USA 1976, 73, 1394.

(26) Terao, T.; Fujii, T.; Onodera, T.; Saika, A. Chem. Phys. Lett. 1984, 107, 145.

(27) Kolbert, A. C.; Grandinetti, P. J.; Baldwin, M.; Pruisner, S. B.; Pines, A. J. Phys. Chem. 1994, 98, 7936.

(28) Krivdin, L. B.; Kalabin, G. A. Prog. in NMR Spect. 1989, $21,293$.

(29) Fung, B. M.; Afzal, J. J. Am. Chem. Soc. 1986, 108, 1107. 
(30) Jameson, C. J. In Multinuclear NMR; J. Mason, Ed.; Plenum Press: New York, 1987; pp 89.

(31) Haeberlen, U. High Resolution NMR in Solids Selective Averaging; Academic Press, 1976.

(32) Büldt, G.; Gally, H. U.; Seelig, J.; Zaccai, G. Nature 1978, 271, 182.

(33) Hester, R. K.; Ackermann, J. L.; Neff, B. L.; Waugh, J. S. Phys. Rev. Lett. 1976, 36, 1081.

(34) Tycko, R.; Dabbagh, G.; Mirau, P. J. Magn. Reson. 1989, 85, 265.

(35) Schmidt-Rohr, K.; Wilhelm, M.; Johansson, A.; Spiess, H. W. Magn. Reson. in Chem. 1993, 31, 352.

(36) Blume, A.; Rice, D. M.; Wittebort, R. J.; Griffin, R. G. Biochemistry 1982, 21, 6220.

(37) Veeman, W. S. Prog. in NMR. Spectro. 1984, 16, 193.

(38) Griffin, R. G.; Powers, L.; Pershan, P. S. Biochemistry 1978, 17, 2718. 


\section{CHAPTER 6}

\section{PHOSPHOCHOLINE STRUCTURE IN THE HEADGROUP AND GLYCEROL BACKBONE}

The motionally-averaged dipolar couplings and chemical shift anisotropies measured in $L_{\alpha}$-phosphocholine are analyzed in this chapter to provide structural information. Some of these NMR couplings confirm known features of phosphocholine conformation such as the headgroup bend, while others provide new constraints on segmental orientations, such as the orientation of the headgroup with respect to the $s n-2$ chain. Combining these averaged NMR couplings, we propose a new conformational model for the core of $\mathrm{L}_{\alpha}$-phosphocholine and compare it with the single-crystal $\mathrm{X}$-ray structure in terms of torsion angles.

\subsection{Acyl Chain Order and Mobility}

The most important property of the phospholipid acyl chains is their high mobility, which gives rise to the liquid crystallinity of the lipid bilayer at physiological temperatures. The acyl chains mainly undergo two types of motions: uniaxial rotational diffusion around the director along with the whole molecule, and trans-gauche isomerizations of individual chain segments. In $\mathrm{L}_{\alpha}$-phosphocholine, both motions are fast on the NMR time scales, as proved by the reduced widths of the dipolar and chemical shift spectra and their axially symmetric lineshapes. For example, we measured the averaged ${ }^{31} \mathrm{P} \mathrm{CSA}(\bar{\delta})$ of fullyhydrated lecithin $(50 / 50 \mathrm{w} / \mathrm{w})$ to be about $-31 \mathrm{ppm}$ (Figure 4.1 and Table 5.2), compared to the rigid-limit CSA $(\delta)$ of $+109 \mathrm{ppm}^{1}$

From the motional averaging of the NMR spectra, we can obtain the upper limit of the motional correlation times $\tau$, since $\tau^{1}$ must be much larger than $\omega_{\text {local }}$ for the spectral averaging to occur. The modulation frequency $\omega_{\text {local }}$ of local spin interactions is much 
smaller than the nuclear Larmor frequency, and normally falls in the kilohertz range. Based on the largest NMR couplings measured in phosphocholine, the ${ }^{2} \mathrm{H}$ quadrupolar splittings, the rotational diffusion and isomerization rates of the acyl chains must be higher than $10^{5} \mathrm{~s}^{-}$ 1. More exact motional rates cannot be obtained from the motionally-averaged spectra, but must be derived instead from NMR relaxation times. ${ }^{2,3}$

What is quantitatively available from the averaged NMR couplings is the segmental order, which can be characterized by the segmental order tensor and bond order parameters. For instance, the $\mathrm{C}-\mathrm{H}$ order parameter profile in Figure 5.6 indicates that acyl chain segments do not have uniform orders: $S_{C H}$ 's have the largest magnitudes $\left(\left|S_{C H}\right|>\right.$ 0.2 ) at the beginning of the chain, reach a plateau in the middle and decrease toward the end of the chain. For the terminal methyl group, $\left|S_{C H}\right|<0.01$. This increasing disorder toward the chain end reflects higher probabilities of gauche conformations for segments further removed from the glycerol moiety or near the center of the bilayer $(\sim n>6)$. In other words, the glycerol moiety is relatively rigid and its adjacent segments $(\sim n<6)$ have high probabilities of adopting the trans conformation.

The bond order parameters can be used to screen and modify conformational models of lecithin. As an example, we consider the $\mathrm{C}-\mathrm{H}$ order parameters of the double bonds in the acyl chain by reexamining a previous calculation of the order tensors of the $C 9$ and $\mathrm{C} 10$ sites. $^{4}$ These order tensors were derived from ${ }^{2} \mathrm{H}$ quadrupolar splittings and infrared dichroism. It was claimed that the ${ }^{2} \mathrm{H}$ couplings must be negative for the $\mathrm{C} 9$ site and positive for the $\mathrm{C} 10$ site, which contradict with our measured $\mathrm{C}-\mathrm{H}$ order parameters of -0.09 and -0.036 , respectively. However, there are several questionable assumptions in the mentioned calculation. First, it was assumed implicitly that the order tensor $\mathbf{S}$ in the segment-fixed coordinate frame has vanishing off-diagonal elements where the corresponding elements of the NMR interaction tensor are zero. This assumption may have resulted from the notion that the order tensor possesses the symmetry of the segment, which is unwarranted as long as that symmetry is not shared by the whole molecule. 
Second, in this calculation, several possible solutions for the order tensor were excluded on the ground that the asymmetry parameter of the $\mathbf{S}$ tensor must be very small in order to agree with the uniaxiality of the averaged NMR interactions in the liquid-crystalline lipid. This notion can be disproved by calculating the segmental order tensors for an internally flexible molecule undergoing, for example, fast exchange between a pair of pseudo-mirror images. One can demonstrate, with a calculation completely analogous to the two-site exchange model encountered in ${ }^{2} \mathrm{H}$ NMR, that the asymmetry parameter of the total segmental order tensor can have any value between zero and one.

It has been observed before that motional rates and order parameters in phospholipids are highly correlated. The trend of the acyl $\mathrm{C}-\mathrm{H}$ order parameters is the same as the trend of the ${ }^{13} \mathrm{C}$ and ${ }^{2} \mathrm{H} \mathrm{T}_{1}$ relaxation times. ${ }^{5}$ However, it may be worthwhile to keep in mind that the segmental order, represented by the order parameters, differs from the segmental mobility, as derived from NMR relaxation times. The order parameters reflect the statistical averaging of segmental conformations, and are influenced by both the motional rates and the segmental orientations with respect to the motional director. For motions fast on the NMR time scales, a small order parameter may result from either largeamplitude near-isotropic motions or motions that are restricted to near-magic-angle orientations. with respect to the director. In comparison, NMR relaxation times probe the rates and mechanisms of motion but not the orientations of the mobile segments. One way of determining motional rates is by measuring the anisotropic ${ }^{2} \mathrm{H} \mathrm{T}_{1}$ relaxation times. It has been estimated from such studies that the rate constants for uniaxial diffusion in phospholipids are on the order of $10^{8} \mathrm{~s}^{-1}$, while the rates for trans-gauche isomerization are approximately $10^{7} \mathrm{~s}^{-1} \cdot{ }^{6}$ Further, it has been demonstrated that by analyzing the lineshapes of partially-relaxed ${ }^{2} \mathrm{H}$ spectra, one can extract detailed motional mechanisms, such as the number of sites involved in discrete jumps. ${ }^{2,3}$

\subsection{Glycerol Rigidity}


The glycerol backbone in phospholipids is the connecting point between the headgroup and the acyl chains. Therefore, it is not surprisingly the most rigid region in the molecule, as suggested by various NMR couplings obtained here. First, the glycerol protons have the shortest $T_{2}$ relaxation times among all protons in lecithin, as indicated by the linewidths of the ${ }^{1} \mathrm{H}$ MAS peaks (Figure 5.9d) and by ${ }^{13} \mathrm{C}$ CPMAS spectra measured with a ${ }^{1} \mathrm{H} \mathrm{T}_{2}$ filter (Figure 5.10). The short ${ }^{1} \mathrm{H} \mathrm{T}_{2}$ times suggest that the glycerol segments have long motional correlation times $\tau_{c}$, which indicate slow motions. In comparison, the $\mathrm{T}_{2}$ of terminal methyl protons is very long, consistent with the flexibility of the acyl chain ends and the three-site jumps of the methyl group. Another evidence for glycerol rigidity is that both the $\mathrm{C}-\mathrm{H}$ bond order parameters and the ${ }^{13} \mathrm{C}$ chemical shift order parameters of the glycerol sites are among the largest in the molecule, as indicated in Figure 5.35.

The relative rigidity of the glycerol backbone suggests that it might be possible to find a single conformation for the glycerol region of $L_{\alpha}$-phosphocholine that reproduces all experimental NMR anisotropies. This is discussed in more detail in section 6.6.

\subsection{Rotational Axes Orientations}

The main symmetry axis in bilayer phospholipids is the axis of uniaxial rotation and is normal to the bilayer surface. Viewed in a segment-fixed frame, the wobbling of the molecule makes the director axis distribute over a region rather than remain at a single orientation. The shape of the director distribution is reflected in the symmetry of the segmental order tensor. An asymmetric order tensor $\left(S_{x x} \neq S_{y y}\right)$ indicates a director distribution that can be approximated by a single biaxial Gaussian or double uniaxial Gaussians (Figure 4.5). An axially symmetric order tensor $\left(S_{x x}=S_{y y}\right)$ indicates a cylindrically symmetric director distribution that can be approximated by a single uniaxial Gaussian. Without assuming internal molecular motions, one cannot be more specific than this distribution-function treatment in describing the rotational axis orientations with respect to the lipid molecules. 
Nevertheless, symmetry considerations, based on the simple fact that the phospholipid acyl chains traverse the bilayer, suggest that the director must be approximately parallel to the long axis of the molecule. Already, this inference suggests the existence of a molecular axis, which can be defined rigorously as the most probable director orientation in the lipid molecule. A well-defined molecular axis is present only if the molecule is rigid, i.e. if the molecular segments are fixed relative to each other. In that case, an order tensor can be transformed from one segmental frame to another by a simple rotation, and diagonalization of the two matrices produces the same principal values and principal-axis orientations. In other words, in a rigid molecule undergoing uniaxial motion, there is one unique and uniaxial segmental order tensor. Moreover, the largest principal value $S_{/ /}$of this order tensor is equal to the order parameter of the molecular axis, $S_{m o l}$. In the following, we assume such a molecular axis, and examine whether our experimental NMR couplings obtained in phosphocholine are consistent with a parallel orientation of the director to the acyl chains and glycerol backbone.

Consider the ${ }^{31} \mathrm{P}-{ }^{13} \mathrm{C}$ dipolar couplings. While most $\mathrm{P}-\mathrm{C}$ couplings are truly longrange, the $\mathrm{P}-\mathrm{C} \alpha$ and $\mathrm{P}-\mathrm{G} 3$ couplings are actually fixed by the $\mathrm{P}-\mathrm{O}-\mathrm{C}$ bond angles and bond lengths. Using the X-ray bond angles and lengths and the averaged NMR couplings (Figure 5.19), we find the $\mathrm{P}-\mathrm{C} \alpha$ and $\mathrm{P}-\mathrm{G} 3$ order parameters to be $\pm 0.23 \pm 0.02$ and $\pm 0.38 \pm 0.04$, respectively. This $P-G 3$ order parameter is one of the largest in phosphocholine, implying that the $\mathrm{P}-\mathrm{G} 3$ vector has one of the highest probabilities of being oriented along the molecular axis. Using the model-independent analysis of the order parameters, we find that the $P-G 3$ vector is preferentially (i. e. probability $>50 \%$ ) oriented at angles smaller than $63^{\circ}$ from the director. In addition, we note that the P-G2 dipolar coupling has a similar magnitude $(144 \mathrm{~Hz})$ to the $\mathrm{P}-\mathrm{C} \alpha$ coupling $(168 \mathrm{~Hz})$, even though the G2 carbon is further removed from the ${ }^{31} \mathrm{P}$ atom than the $\mathrm{C} \alpha$ carbon. This indicates that the $\mathrm{P}-\mathrm{G} 2$ order parameter is larger than the $\mathrm{P}-\mathrm{C} \alpha$ one, i.e. the $\mathrm{P}-\mathrm{G} 2$ vector is more parallel with the molecular axis than the $\mathrm{P}-\mathrm{C} \alpha$ vector. 
We find that most glycerol and acyl $\mathrm{C}-\mathrm{H}$ bond order parameters are negative in phosphocholine (Figures 5.6 and 5.35). This means that the $\mathrm{C}-\mathrm{H}$ bonds are oriented more perpendicular than parallel to the director. In other words, the $\mathrm{C}-\mathrm{C}$ backbone orientations are more parallel to the director. Take for example the G3 segment, whose $\mathrm{C}-\mathrm{H}$ bonds have order parameters of -0.22 . This would correspond to an angle of $\theta=64^{\circ}$ if the G3$\mathrm{H}_{\mathrm{G} 3}$ bonds assume a single orientation with the director, as $P_{2}\left(\cos 64^{\circ}\right)=-0.22$. However, since the segment is mobile, according to the model-independent analysis of bond order parameters, the $\mathrm{G} 3-\mathrm{H}_{\mathrm{G} 3}$ bonds are oriented at angles larger than $50^{\circ}$ from the director in more than $50 \%$ of the time. The probability for these bonds to be oriented at angles smaller than $30^{\circ}$ is smaller than $25 \%$. Further, if we make the realistic assumption that any maximum in the orientation distribution has a standard deviation of at least $10^{\circ}$ (but still not making any assumptions about the conformations of the molecule), then we find that the $\mathrm{G} 3-\mathrm{H}_{\mathrm{G} 3}$ bonds are very unlikely $(<25 \%)$ to be oriented at angles smaller than $51^{\circ}$ (Figure 4.8).

In conclusion, our experimental NMR couplings are consistent with a $L_{\alpha-}$ phosphocholine structure in which the acyl chains and the glycerol backbone are approximately parallel to the bilayer normal.

Uniaxial rotational diffusion is not the only motion in the phosphocholine lipids. Another motion, well studied but still incompletely understood, is the exchange of the phosphocholine headgroup within pairs of mirror-symmetric conformations. This motion was proposed originally to explain the degeneracy of the geminal ${ }^{2} \mathrm{H}$ quadrupolar splittings of the headgroup segments. ${ }^{7}$ The two enantiomeric conformers were constructed by inverting the signs of the torsion angles $\alpha_{1}(\mathrm{G} 3-O)$ to $\alpha_{5}(\mathrm{C} \alpha-\mathrm{C} \beta)$, with the assumption that the G2-G3 bond preceding $\alpha_{1}$ is collinear with the director axis. This assumption places the molecular rotation axis in the mirror plane of the enantiomeric conformers, and is necessary for ensuring the degeneracy of the geminal $\mathrm{C}-\mathrm{H}$ bonds. In addition, the proposed coincidence of the G2-G3 bond with the rotational axis accounts for the near 
degeneracy of the ${ }^{2} \mathrm{H}$ couplings in the $\mathrm{G} 3$ segment $(27 \mathrm{kHz}$ and $29 \mathrm{kHz}$ ) by invoking the unhindered rotation of the $\mathrm{G} 3-\mathrm{H}_{\mathrm{G} 3}$ bonds around the $\mathrm{G} 2-\mathrm{G} 3$ axis. In other words, according to this model, the G3 site needs not participate in the enantiomeric exchange for its $\mathrm{C}-\mathrm{H}$ bond order parameters to be equivalent.

This proposed $\mathrm{G} 2-\mathrm{G} 3$ bond orientation is challenged by the $\mathrm{H}_{\mathrm{G} 3}-\mathrm{H}_{\mathrm{G} 3}$ coupling we measured (Figure 5.13 and Table 5.2). According to the tetrahedral geometry, a parallel G2-G3 orientation to the molecular rotation axis would make the $\mathrm{H}_{\mathrm{G} 3}-\mathrm{H}_{\mathrm{G} 3}$ vector perpendicular to it. Correspondingly, the $\mathrm{H}_{\mathrm{G} 3}-\mathrm{H}_{\mathrm{G} 3}$ dipolar coupling would be scaled by $P_{2}\left(\cos 90^{\circ}\right)=-1 / 2$, in addition to a factor of 0.66 from the molecular order parameter. Since the distance between the geminal protons is fixed by the segment, the rigid-lattice H$\mathrm{H}$ dipolar coupling is known. This $\mathrm{H}_{\mathrm{G} 3}-\mathrm{H}_{\mathrm{G} 3}$ orientation would then result in an averaged $\mathrm{H}_{\mathrm{G} 3}-\mathrm{H}_{\mathrm{G} 3}$ coupling of more than $10 \mathrm{kHz}$, which is inconsistent with our experimental value of $6.6 \mathrm{kHz}$. Conversely, if one assumes a molecular order parameter of 0.66 and internal rotation of the headgroup, then the measured $\mathrm{H}_{\mathrm{G} 3}-\mathrm{H}_{\mathrm{G} 3}$ coupling would correspond to an order parameter $\left|S_{H H}\right|=0.2 \pm 0.02$, which indicates an angle of $68^{\circ}$ or $44^{\circ}$ between the rotation axis and the $\mathrm{H}_{\mathrm{G} 3}-\mathrm{H}_{\mathrm{G} 3}$ vector. This would require the $\mathrm{G} 2-\mathrm{G} 3$ bond to deviate from the bilayer normal. In conclusion, to meet the required degeneracy of the G3$\mathrm{H}_{\mathrm{G} 3}$ bonds, the $\mathrm{G} 3$ methylene group must either participate in the enantiomeric exchange, or be oriented in such a fortuitous way that its $\mathrm{C}-\mathrm{H}$ bonds have nearly identical $S_{C H}$.

Determination of the orientation of the torsion bond preceding the flexible headgroup region is a complex problem. Both the geminal $\mathrm{C}-\mathrm{H}$ degeneracies and various other NMR dipolar couplings must be reproduced. Although the strength of the $\mathrm{H}_{\mathrm{G} 3}-\mathrm{H}_{\mathrm{G} 3}$ coupling challenges the hypothesis of a special $G 2-G 3$ bond orientation, in the new $L_{\alpha}$ phosphocholine conformation model we propose based on more than 20 NMR anisotropies, the molecular rotation axis is nevertheless constrained to an orientation close to the $\mathrm{G} 2-\mathrm{G} 3$ bond direction. 


\subsection{Headgroup and Acyl Chain Bends}

The bend of the phosphocholine headgroup from the glycerol backbone and acyl chains has been deduced unambiguously from neutron diffraction, and is also present in the single-crystal X-ray structure. In the following, we show that our experimental NMR data confirm this bend.

The most direct evidence for the headgroup bend is provided by the long-range $\mathrm{C}$ $\mathrm{H}$ dipolar couplings, which are obtained qualitatively from ${ }^{13} \mathrm{C} /{ }^{1} \mathrm{H}$ chemical shift correlation spectra (Figures 5.8 and 5.9). The intensity differences among the $\mathrm{CP}$ signals show that the $\mathrm{CO} 2$ carbon couples to the $\mathrm{H} \alpha$ protons most strongly. Generally, dipolar coupling strengths are related to the lengths and orientations of the internuclear vectors according to equation (2.5). For motionally-averaged dipolar couplings, the relation is modified to

$$
\bar{\delta}_{C H}=-\frac{\mu_{0}}{4 \pi} \hbar \gamma_{C} \gamma_{H} \cdot\left\langle\frac{1}{r_{C H}^{3}} \frac{1}{2}\left(3 \cos ^{2} \theta_{C H}-1\right)\right\rangle,
$$

where $r_{C H}$ is the internuclear distance and $\theta_{C H}$ is the instantaneous angle between the $\mathrm{C}-\mathrm{H}$ vector and the motional axis. The relatively strong $\mathrm{CO} 2-\mathrm{H} \alpha$ dipolar coupling thus implies that both the $\mathrm{CO} 2-\mathrm{H} \alpha$ distance and its average orientation must be favorable for producing an appreciable $\mathrm{C}-\mathrm{H}$ coupling: the $\mathrm{CO} 2-\mathrm{H} \alpha$ distance must be sufficiently small and $\theta_{C H}$ must be close to $0^{\circ}$. This suggests that the $\mathrm{H} \alpha$ protons are located directly above the $\mathrm{CO} 2$ carbon, if the director is assumed to point up. In comparison, the $\mathrm{H} \beta$ signal in the HETCOR spectrum (Figure 5.9b) is much weaker than H $\alpha$. This cannot be explained merely by a mobility difference, since the $\alpha$ and $\beta$ segments are directly bonded. Therefore, the different $H \alpha$ and $H \beta$ intensities suggest that $H \beta$ 's are on average more distant from the $\mathrm{CO} 2$ carbon than $\mathrm{H} \alpha$ 's, and/or $\mathrm{H} \beta$ 's are not located directly above it. Similarly, the low intensity of the $\mathrm{H} \gamma$ signal, which is especially remarkable since there are 
4.5 times more $\mathrm{H} \gamma$ protons than $\mathrm{H} \alpha$ or $\mathrm{H} \beta$ protons, can be attributed to the large distances of $\mathrm{H} \gamma$ 's from the $\mathrm{CO} 2$ carbon, as well as to the additional rotational freedom of the methyl groups around the $\mathrm{C} \beta-\mathrm{N}$ and $\mathrm{N}-\mathrm{C} \gamma$ bonds.

The relative proximity between the $\mathrm{CO} 2$ carbon and the $\mathrm{H} \alpha$ protons is only possible if the phosphocholine headgroup bends from the glycerol backbone toward the $s n-2$ chain and the beginning of the $s n-2$ chain also bends toward to the headgroup. These features are found in the folded conformation of molecule B in the crystal structure of DMPC (Figure 6.1). ${ }^{8}$ In the other conformation, molecule $\mathrm{A}$, the headgroup bends from the glycerol backbone but away from the $s n-2$ chain. Thus structure A exhibits a larger distances between the $\mathrm{CO} 2$ carbon and Ho's and an oblique orientation of the corresponding internuclear vectors with the bilayer normal. Both factors are unfavorable for producing a significant $\mathrm{C}-\mathrm{H}$ dipolar coupling. In conclusion, the observed relative strengths of the long-range $\mathrm{C}-\mathrm{H}$ dipole couplings indicate qualitatively that a folded-back headgroup conformation similar to molecule B in the crystal is significantly populated in the liquidcrystalline phase.

Further evidence for the headgroup bend in $\mathrm{L}_{\alpha}$-phosphocholine is provided by the signs of various NMR anisotropies at the $\mathrm{C} \alpha$ segment. In the $\mathrm{C}-\mathrm{H}$ order profile (Figure 5.6), the $\mathrm{C} \alpha-\mathrm{H} \alpha$ bond order parameter has a positive sign, which is opposite to the negative sign of the neighboring $G 3$ and $C \beta$ sites and most other methylene groups. Considering the large difference between the $\mathrm{C} \alpha$ and G3 order parameter values, the sign inversion of the $\mathrm{C} \alpha-\mathrm{H} \alpha$ coupling is especially significant. Additionally, our ${ }^{13} \mathrm{C}$ SAS spectra (Figure 5.30) indicate that the C $\alpha$ carbon has a positive CSA, in contrast to the negative CSAs of most other carbons. The sign anomalies of these C $\alpha$ NMR anisotropies point to an unusual orientation of the $\mathrm{C} \alpha$ segment, which can be satisfied by the headgroup bend from the glycerol backbone at the phosphate junction: as the first segment after the phosphate and into the headgroup, the $\alpha$ segment would be most affected by such a conformational change. 
With a model-independent analysis of the bond order parameters, the $\mathrm{C} \alpha-\mathrm{H} \alpha$ order parameter $\left(S_{C H}=+0.08\right)$ can be interpreted quantitatively: a probability upper limit of $100 \%$ is reached when the $\mathrm{C} \alpha-\mathrm{H} \alpha$ bonds are oriented between $0^{\circ}$ and $51.5^{\circ}$ relative to the director. Although this does not exclude the possibility that the $\mathrm{C} \alpha-\mathrm{H} \alpha$ bonds are oriented at the complementary angles of $51.5^{\circ}$ to $90^{\circ}$, it nevertheless denotes a small probability of such orientations. The $\mathrm{C} \alpha-\mathrm{H} \alpha$ bond orientations can be compared with the $\mathrm{G} 3-\mathrm{H}_{\mathrm{G} 3}$ orientations, which are preferentially between $50^{\circ}$ and $90^{\circ}$ from the director.

The model-independent analysis can also be applied to the averaged chemical shift anisotropies, as discussed in Chapter 4 . For example, the ${ }^{31} \mathrm{P}$ chemical shift anisotropy has $\bar{\delta} / \delta=-0.25$ and $\eta=0.56$. This indicates that the vector connecting the non-esterified oxygens of the phosphate unit, the $\sigma_{z z}$ axis of the ${ }^{31} \mathrm{P}$ chemical shift tensor, is preferentially oriented between $40^{\circ}$ and $140^{\circ}$ from the director.

Further information about the headgroup orientation comes from the $\mathrm{P}-\mathrm{C}$ couplings. As mentioned before, the $\mathrm{C} \alpha$ carbon, which is one bond closer to the ${ }^{31} \mathrm{P}$ spin than the $\mathrm{G} 2$ carbon, has a comparable $\mathrm{P}-\mathrm{C}$ coupling as the $\mathrm{P}-\mathrm{G} 2$ coupling. This means that the $\mathrm{P}-\mathrm{C} \alpha$ vector has a much smaller $S_{P C}$ than the $\mathrm{P}-\mathrm{G} 2$ vector, and is again consistent with the headgroup bend from the glycerol backbone and acyl chains.

\subsection{Enantiomeric Headgroup Conformations}

In section 6.3 , the model of headgroup exchange between two enantiomers, proposed ${ }^{7}$ to explain the persistent approximate degeneracy of the geminal ${ }^{2} \mathrm{H}$ couplings in the headgroup, was discussed. We now examine the validity of this model, using motionally-averaged NMR anisotropies as structural constraints. We then explore alternative mechanisms that reproduce similar degeneracies in other methylene segments.

\section{Evidence of Headgroup Exchange}


Several NMR couplings confirm that the geminal $\mathrm{C}-\mathrm{H}$ bonds in the headgroup segments are indeed degenerate. First, only one $\mathrm{C}-\mathrm{H}$ dipolar coupling is observed for the two $\mathrm{C}-\mathrm{H}$ bonds of each methylene group. Not only the magnitudes but also the signs of these geminal $\mathrm{C}-\mathrm{H}$ couplings are identical, as found by the 2D PDLF experiments. Second, only one P-H splitting is observed for the G3 and $\mathrm{C} \alpha$ sites. Third, the $\mathrm{C} \alpha$ and G3 sites show small non-geminal $\mathrm{H}-\mathrm{H}$ dipolar couplings. The highly-resolved $\mathrm{P}-\mathrm{H} / \mathrm{H}-\mathrm{H}$ spectrum (Figure 5.13) indicates that the geminal $\mathrm{H}-\mathrm{H}$ couplings in these sites dominate by at least a factor of four over the non-geminal ones. If the headgroup were rigid and only underwent rotations around the molecular long axis, then the geminal and nou-geminal dipolar couplings would be comparable. If, however, a motional process exists that interchanges the geminal protons, then intersegmental $\mathrm{H}-\mathrm{H}$ couplings would be averaged whereas the intrasegmental geminal H-H couplings would remain unaffected.

The enantiomeric headgroup conformations of phosphocholine are present in the crystal as well. The X-ray crystal structure of DMPC shows two molecules with different headgroup orientations in an asymmetric unit cell. ${ }^{8}$.The torsion angles $\alpha_{i}(i=1,2, . .5)$ in molecule B have opposite signs from those in molecule A (Figure 6.1). Table 6.1 lists the torsion angles found in single-crystal phosphocholine. It is conceivable that the double headgroup conformations are simply carried over from the crystal to the fluid phase. However, in the liquid-crystalline phase, the headgroup may exchange between these two conformations while in the solid it cannot.

\begin{tabular}{c|cccccccccccc}
\hline & $\alpha_{1}$ & $\alpha_{2}$ & $\alpha_{3}$ & $\alpha_{4}$ & $\alpha_{5}$ & $\theta_{1}$ & $\theta_{3}$ & $\beta_{1}$ & $\beta_{2}$ & $\beta_{3}$ & $\beta_{4}$ & $\gamma_{1}$ \\
\hline $\mathrm{A}$ & 162 & 68 & 63 & 139 & -51 & 58 & -176 & 76 & 180 & -87 & 63 & -173 \\
$\mathrm{~B}$ & 170 & -76 & -46 & -161 & 64 & 169 & 175 & 124 & 169 & -126 & 63 & 103 \\
\hline
\end{tabular}

Table 6.1 Torsion angles in the headgroup and glycerol backbone of single-crystal phosphocholine. $^{8}$ 


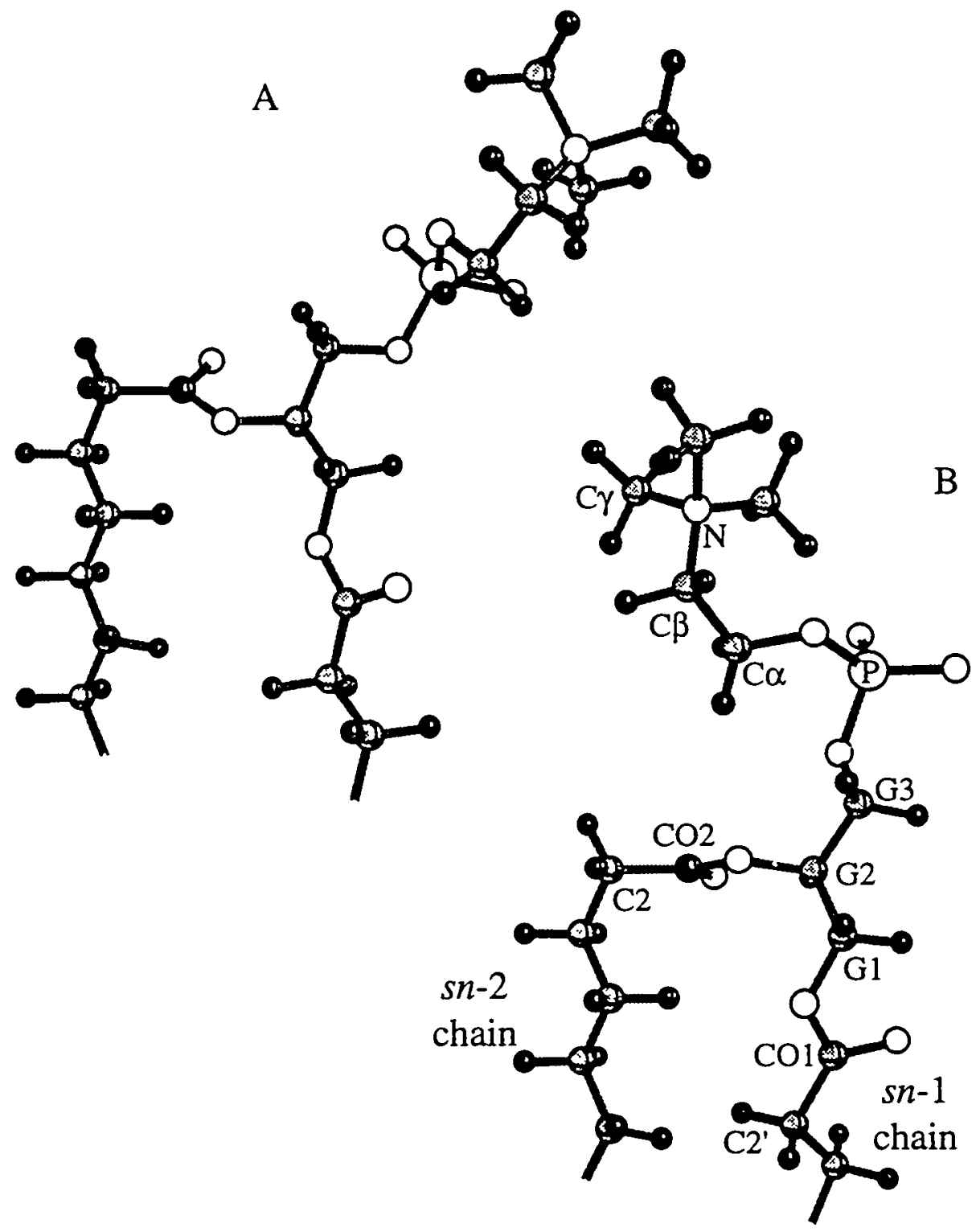

Figure 6.1 Two DMPC conformations in a crystal unit cell. ${ }^{8}$ The headgroup bends away from the acyl chains in conformation $A$, but hangs close above the $s n-2$ chain in conformation B. The nomenclature for the segments is indicated on molecule B. 


\section{Populations of Headgroup Conformers}

Let us assume that the crystal conformations $A$ and $B$ are the enantiomers in the $L_{\alpha}$ phase, and examine to which extent they can produce a valid enantiomeric exchange model for the fluid phosphocholine. Based on the long-range $\mathrm{C}-\mathrm{H}$ dipolar couplings, we have concluded that conformation B must be significantly populated in $\mathrm{L}_{\alpha}$-phosphocholine. However, this qualitative conclusion does not rule sut the presence of type-A conformations. The dipolar couplings would be reduced by type- $\mathrm{A}$ conformations, but could still be dominated by the strong couplings in the folded-back headgroup in structure B. Therefore, we need to estimate the relative population of type-A and type-B conformations.

To solve this problem, we need to constrain the phosphocholine headgroup orientation more stringently by utilizing the NMR anisotropies reported in the literature as well as those measured here. In particular, the ${ }^{31} \mathrm{P}-13 \mathrm{C}$ dipolar couplings involving the $\mathrm{CO} 2$ site $(50 \mathrm{~Hz})$ and the glycerol G3 site $(240 \pm 20 \mathrm{~Hz})^{9}$ are relatively large and serve as strong constraints. In our analysis, a single glycerol conformation is considered, since it is sufficient to capture the essential features of type-A and type-B conformations. The overall wobbling of the molecule is taken into account by a molecular order parameter of $0.6 .^{10}$ To be general, various orientations of the bilayer normal with respect to the G2-G3 bond are considered.

Our analysis indicates that the NMR couplings for any conformation similar to molecule A deviate significantly from the experimental couplings. The magnitude of the P$\mathrm{CO} 2$ coupling would be smaller than $12 \mathrm{~Hz}$, and that of the P-G3 coupling less than 160 Hz. In contrast, conformation B yields $\mathrm{P}-\mathrm{C}$ couplings similar to (0.4-1.3 times) the experimental values, depending on the exact orientation of the bilayer normal. Although no choice of the bilayer normal in conformation B could reproduce all known NMR dipolar couplings for the glycerol backbone, a structure similar to conformation B but characterized by an even stronger bend of the headgroup yields an acceptable solution. The existence of 
this structure (Figure 6.2), which is discussed in the next section, corroborates the predominance of type-B conformations with a headgroup hanging over the $s n-2$ chain in the phosphocholine bilayers.

To estimate the extent to which type-B conformations dominate the structure of $\mathrm{L}_{\alpha}$ phosphocholine, we need to determine whether a type-B structure can be found in which both $\mathrm{P}-\mathrm{CO} 2$ and $\mathrm{P}-\mathrm{G} 3$ couplings exceed the experimental values significantly. If such a structure could be found, then type-A structures could be invoked to reduce the average couplings to the measured values. Our analysis shows that, due to an angle of $\sim 40^{\circ}$ between the $\mathrm{P}-\mathrm{CO} 2$ and $\mathrm{P}-\mathrm{G} 3$ vectors, no structure exhibits large $\mathrm{P}-\mathrm{C}$ couplings for both sites. To generate a large $\mathrm{P}-\mathrm{G} 3$ coupling, the phosphorus atom must be located above the G3 carbon, which removes it to a larger distance and a more oblique angle from the $\mathrm{CO} 2$ carbon. The conformation with the largest combined $\mathrm{P}-\mathrm{C}$ couplings exhibits about $55 \mathrm{~Hz}$ and $280 \mathrm{~Hz}$ for the $\mathrm{P}-\mathrm{CO} 2$ and $\mathrm{P}-\mathrm{G} 3$ couplings, respectively. With an experimental value of $50 \pm 8 \mathrm{~Hz}$ for the $\mathrm{P}-\mathrm{CO} 2$ coupling, ${ }^{9}$ this allows for only $15 \%( \pm 15 \%)$ of conformation A. Therefore, structures similar to $\mathrm{B}$, in which the $\theta_{1}$ torsion is close to trans to make the headgroup bend toward the $s n-2$ chain, are the predominant conformations in the liquidcrystalline phosphocholine bilayer.

If order tensors of various segments can be solved with additional NMR couplings, we can test the validity of the two-conformation model by applying the double-Gaussian approximation to the full orientation distribution. If the two-site model is correct, the widths of the Gaussians must be the same for all segments involved. Then, by aligning the maxima of the Gaussians and the $\mathrm{C}-\mathrm{C}$ bond directions for the adjacent segments, the torsion angle is constrained to four values, namely pairs of values with opposite signs. One of these pairs is the correct solution. On the other hand, complex internal dynamics of the molecule is proved by highly non-uniaxial order tensors, as well as variation of the Gaussian peak widths in the model distribution. For example, a systematic increase of the Gaussian width parameter towards the chain end may suggest a mobility gradient. 
In conclusion, enantiomeric headgroup conformations similar to molecules A and B in the crystal do not meet the constraints of the motionally-averaged NMR anisotropies simultaneously. Type-B conformations are predominant in $L_{\alpha}$-phosphocholine. Therefore, if the headgroup exchanges between two mirror-image conformations, such conformations must differ in its details from molecule B. It is conceivable that both headgroup enantiomers are out of the plane of the acyl chains and still remain closer to the $s n-2$ chain than to the $s n-1$ chain. This scenario would almost certainly involve modification of the starting torsion angle of the flexible region and of the orientation of the molecular rotation axis.

\section{General Requirements for $\mathrm{C}-\mathrm{H}$ Degeneracies in Liquid-Crystalline Systems}

Many questions are left unanswered by Seelig's model of the headgroup exchange. For example, what is the number of the exchanging conformers? One cannot exclude the possibility of $2 n(n \gg>1)$ conformers. Why should a simultaneous inversion of all torsion angles occur? More puzzling still is the often-neglected fact that the geminal proton degeneracy is also observed in most acyl chain segments, for which neither the torsion angle inversion nor a special bond orientation has been invoked. The implicit assumption seems to be that trans-gauche isomerization sufficiently equalizes or averages the geminal $\mathrm{C}-\mathrm{H}$ bonds.

The phenomena of degenerate $\mathrm{C}-\mathrm{H}$ bonds in methylene groups of many anisotropically mobile systems, including nematic liquid crystals, suggest a more general mechanism for the molecular motions that cause such degeneracy. In response to this, we propose a set of general symmetry requirements for $\mathrm{C}-\mathrm{H}$ degeneracies in alkyl chain methylene groups.

For the exchange to produce degenerate $S_{C H}$ 's, the director distribution seen in a segment-fixed frame must be symmetric with respect to the plane bisecting the $\mathrm{H}-\mathrm{C}-\mathrm{H}$ bond angle. This implies that one of the principal axes of the segmental order tensor must 
be oriented along the geminal $\mathrm{H}-\mathrm{H}$ vector. This condition is necessary regardless of the motional mechanisms.

Consider the case of a molecule interconverting, as a whole, among an even number of conformations with equal probabilities. The torsion potentials must be symmetric with respect to the equivalent conformers in order to produce degenerate $S_{\mathrm{CH}}$ 's. For a linear and achiral molecule, which has a symmetric interaction potential by definitior, if the probability of the molecular orientation relative to the director is determined solely by the director orientation in the molecule, then the director must be in the mirror plane of two equivalent orientations. When the molecular motion is fast on the measurement time scale, then each conformation always has a mirror-symmetric counterpart. Averaging between these conformational pairs gives rise to degenerate $S_{C H}$ 's.

If the molecule has a chiral center, such as the G2 carbon in phosphocholine, but possesses one or more flexible regions (e.g. the acyl chains of phosphocholine) that are effectively decoupled from the chiral region of the molecule (e.g. by the bulky carboxyl segments), then the above criterion for degenerate $S_{C H}$ 's also holds for the flexible regions of the molecule. Again, the flexible segments must be linear and achiral, so that torsion angle potentials are symmetric.

For molecules with a chiral center outside the flexible region, the molecular rotation axis must lie in the plane formed by bonds $i-1$ and $i$, where $i$ is the first torsion angle in the interconverting region. This ensures that the molecular rotation axis lies in the symmetry plane of the mirror conformations. Seelig's model of the headgroup exchange is a special case for this rule, since a rotation axis along the G2-G3 bond certainly and trivially lies in the mirror plane defined by the G2-G3 bond (a fixed torsion) and the G3-O bond (the first interconverting torsion). However, the molecular rotation axis does not have to coincide with either bond as long as it is in the G2-G3-O plane.

These general considerations broaden our perspective in understanding the true mechanism of the geminal proton degeneracy in $\mathrm{L}_{\alpha}$-phosphocholine. They suggest that the 
$S_{C H}$ degeneracy in the headgroup may be less special than considered so far. An indirect piece of evidence for this is provided by the chiral segment $\mathrm{G} 2$, which has a crowded and sterically-unfavorable three-fold branching to three long chains. This chirality breaks the torsion symmetry of the adjacent G1 and G3 sites, and non-identical $S_{C H}$ 's are indeed observed for the $\mathrm{G} 1-\mathrm{H}_{\mathrm{G} 1}$ bonds. In conjunction with the geminal $\mathrm{H}_{\mathrm{G} 3}-\mathrm{H}_{\mathrm{G} 3}$ coupling, this strongly suggests that the $S_{C H}$ degeneracy at the G3 site is accidental.

\subsection{Single-Conformation Model for Phosphocholine Core}

So far, we have considered the overall structure of phosphocholine in terms of the distinction between conformations A and B. Taking into account more and more NMR couplings from the literature, we are led to a more and more specific molecular structure. This prompt us to investigate whether there is a single conformation for the "core" of the molecule, i.e. the glycerol backbone and its adjacent segments, which fulfills all known NMR couplings. Twenty anisotropic NMR couplings from our measurements and the literature are considered, in particular the $\mathrm{P}-\mathrm{G} 3, \mathrm{P}-\mathrm{CO} 2, \mathrm{P}-\mathrm{G} 2, \mathrm{P}-\mathrm{G} 1, \mathrm{P}-\mathrm{CO} 1,{ }^{9,11} \mathrm{G} 3-$ $\mathrm{H}_{\mathrm{G} 3}, \mathrm{G} 2-\mathrm{H}_{\mathrm{G} 2}{ }^{12,13}$ and $\mathrm{H}_{\mathrm{G} 3}-\mathrm{H}_{\mathrm{G} 3}{ }^{11}$ dipolar couplings, and the chemical shift anisotropies of ${ }^{13} \mathrm{CO}^{9,14}$ and ${ }^{31} \mathrm{PO}_{4}{ }^{1}$ segments. The overall wobbling of the molecule is taken into account by a molecular order parameter of $0.6 .^{10}$

Indeed, we find such a single conformation for the phosphocholine core (Figure 6.2). At the central $\mathrm{G} 2$ site, the bilayer normal forms an angle of $35^{\circ} \pm 10^{\circ}$ with the G2-G3 bond, and $109^{\circ} \pm 7^{\circ}$ with the $\mathrm{G} 2-\mathrm{H}_{\mathrm{G} 2}$ bond, as determined by the $\mathrm{G} 2-\mathrm{H}_{\mathrm{G} 2}$ order parameter. ${ }^{13,15}$ A parallel orientation of this molecular axis to the G2-G3 bond is excluded based on the fact that it does not allow for any ${ }^{31} \mathrm{P}-\mathrm{G} 3$ couplings larger than $160 \mathrm{~Hz}$ and yields too large a $\mathrm{H}_{\mathrm{G} 3}-\mathrm{H}_{\mathrm{G} 3}$ coupling. The $\mathrm{G} 1-\mathrm{G} 2-\mathrm{G} 3-\mathrm{O}$ torsion is trans $\left(\theta_{1}=185^{\circ}\right)$, which produces a bent headgroup similar to conformation B, while the O-G1-G2-G3 torsion is definitely not trans $\left(\theta_{3}=265^{\circ}\right)$, since the $\mathrm{C}-\mathrm{H}$ bond order parameters for the $\mathrm{Gl}$ and G3 methylene groups are considerably different. The G2-G3-O-P torsion angle 
$\left(\alpha_{1}=110^{\circ}\right)$, in conjunction with $\theta_{1}$, is crucial for positioning the phosphorus atom above the G2 site, in order to fulfill the constraints of the large number of P-C couplings,, 9.11 especially those involving the $\mathrm{CO} 2$ and G3 carbons. The G3-O-P-O torsion $\left(\alpha_{2}=170^{\circ}\right)$ is less stringently defined than the $\alpha_{1}$ torsion, as it is constrained only by the ${ }^{31} \mathrm{P}$ chemical shift anisotropy. ${ }^{1,16,17}$ Although the trans conformation of $\alpha_{2}$ deviates from the X-ray structure, ${ }^{8}$ the bend of the headgroup is still maintained. As a result, the orientation of the $\alpha$ segment differs from that of other methylene groups, as suggested by various NMR order parameters. Further torsion angles in the headgroup $\left(\alpha_{3}, \alpha_{4}\right.$, and $\left.\alpha_{5}\right)$ cannot be given, because the consistently small order parameters of the $C \alpha$ and $C \beta$ segments indicate high mobilities, which make a single-conformation description of the end of the headgroup inappropriate. In the acyl chain region, the structure in Figure 6.2 reproduces the ${ }^{13} \mathrm{C}$ chemical shift anisotropies of $\mathrm{CO} 1$ and $\mathrm{CO} 2$ (Table 5.1). The $\beta_{1}$ and $\beta_{2}$ torsions on the $s n-2$ chain are $145^{\circ}$ and $110^{\circ}$, respectively, while the $\gamma_{1}$ and $\gamma_{2}$ torsions on the $s n-1$ chain assume gauche $\left(290^{\circ}\right)$ and trans $\left(180^{\circ}\right)$ conformations. Further down the chains, the C2 and $\mathrm{C} 2$ ' segments exhibit different orientations, because their $\mathrm{C}-\mathrm{H}$ order parameters ${ }^{18}$ and ${ }^{13} \mathrm{C}$ chemical shift anisotropies differ substantially. The rest of the acyl chains is represented by trans conformations for convenience. A realistic structure, as characterized by the NMR order parameter profile, involves enhanced internal dynamics with an increasing number of gauche conformations toward the chain ends. ${ }^{19}$

The rigid-backbone model presented here (Figure 6.2) successfully reproduces the NMR couplings in the core of the phosphocholine molecule in the liquid-crystalline state. Although it does not exclude a more complex structure with interchanging conformations, a single-conformation model, if it fits all known NMR data and correctly predicts other NMR couplings, provides a simple approximation to the structure, which may be more valuable than more complicated multi-conformation models for further lipid research. In any case, the model can serve to visualize structural constraints imposed by the NMR couplings. In 


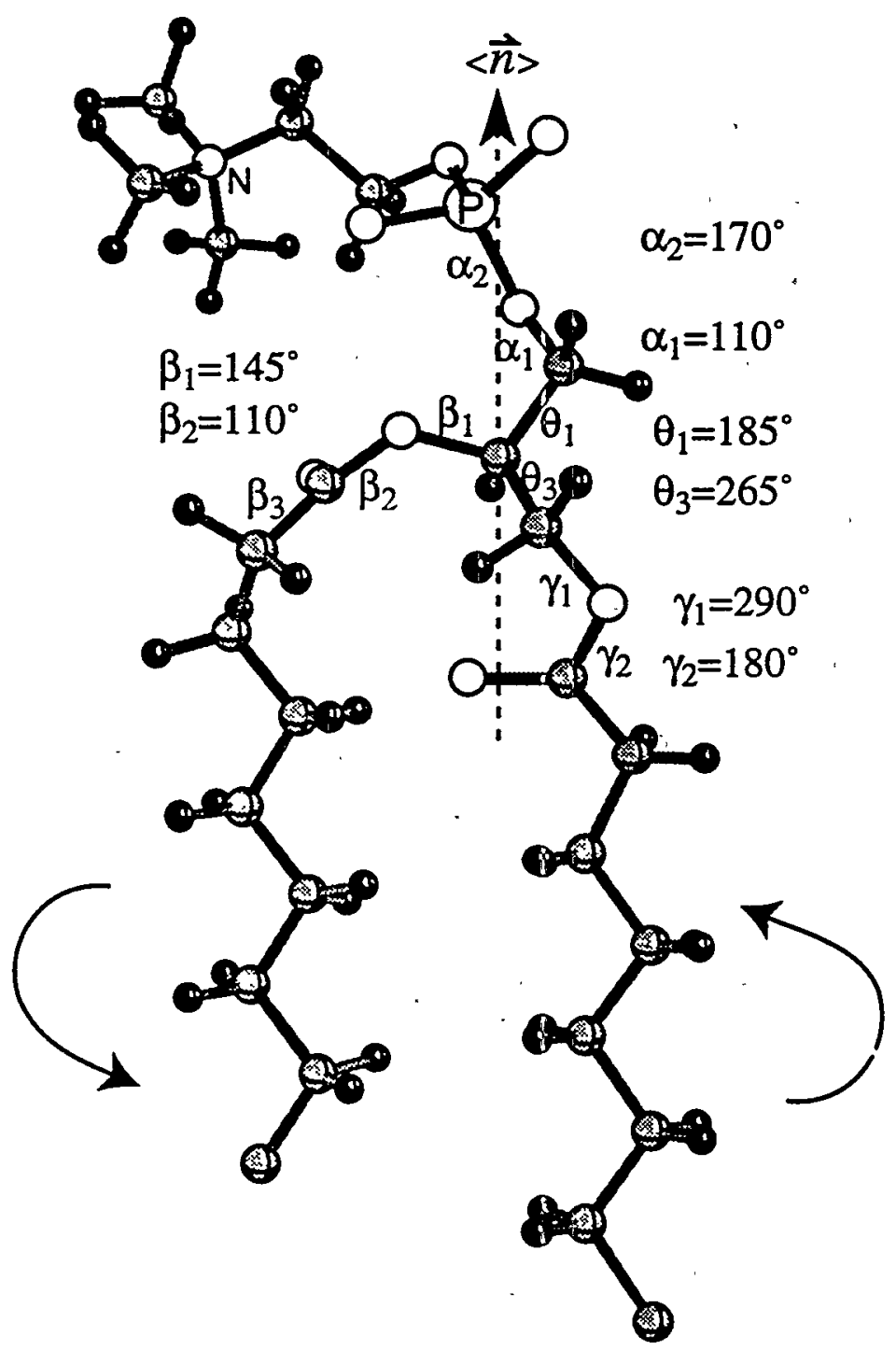

Figure 6.2 A new structure proposed for the core of $L_{\alpha}$-phosphocholine. It is consistent with 20 known NMR dipolar couplings and chemical shift anisotropies. A single backbone conformation is assumed. 
conclusion, based on experimental NMR couplings and model-building, the conformation of $\mathrm{L}_{\alpha}$-phosphocholine is found to be dominated by one type of structure. It can be approximated by a rigid conformation in the core of the molecule and exhibits a bent-back headgroup.

\subsection{Order Tensor of $\beta$ Segment}

As the final topic of this chapter, we discuss the conformation of the $\beta$ segment in phosphocholine using the order tensor formalism described in Chapter 4. The $\beta$ segment has the largest number of couplings necessary for calculating the order tensor. First, only one $\mathrm{C}-\mathrm{H}$ dipolar coupling and one ${ }^{2} \mathrm{H}$ quadrupolar splitting is observed for the $\gamma$-methyl groups. This indicates a fast motion of the $\mathrm{N}\left(\mathrm{CH}_{3}\right)_{3}$ moiety around the $\mathrm{C} \beta-\mathrm{N}$ bond with at least $C_{3}$ symmetry. Based on $X$-ray bond angles $\left(113^{\circ}\right.$ for $C \beta-N-C \gamma$ and $107^{\circ}$ for $N-C \beta-$ $\mathrm{H})$ and sign-sensitive PDLF spectra, we deduce that the $\mathrm{C} \beta-\mathrm{N}$ bond order parameter is $+0.1 \pm 0.02$. Thus, we have three segment-fixed order parameters for the $C \beta$ methylene group: the $\mathrm{C}-\mathrm{H}$ and $\mathrm{C}-\mathrm{N}$ bond order parameters of -0.04 and +0.1 , respectively, and the geminal $\mathrm{H}-\mathrm{H}$ order parameter of \pm 0.05 . Taking into account the degeneracy of the $\mathrm{C}-\mathrm{H}$ directions, these order parameters should be sufficient for characterizing the order tensor of the $\beta$ segment. However, since the sign of the $\mathrm{H}-\mathrm{H}$ coupling is not known, two possible solutions are present. One solution has the principal values $(0.12 \pm 0.02,-0.05 \pm 0.01$, $-0.07 \pm 0.02)$, and the other $(-0.22 \pm 0.03,0.05 \pm 0.01,0.17 \pm 0.03)$. The second set of

principal values is significantly larger than the experimental order parameters. This serves as a reminder that it is not possible to conclude, based on a few small order parameters, that a segment is more mobile than others with larger couplings.

To complete the order tensor analysis, more segment-fixed order parameters must be determined. These may be obtained from intersegmental dipolar $\mathrm{C}-\mathrm{H}$ couplings or chemical shift anisotropies. 


\section{References}

(1) Griffin, R. G.; Powers, L.; Pershan, P. S. Biochemistry 1978, 17, 2718.

(2) Witterbort, R. J.; Olejniczak, E. T.; Griffin, R. G. J. Chem. Phys. 1987, 86, 5411.

(3) Siminovitch, D. J.; Ruocco, M. J.; Olejniczak, E. T.; Gupta, S. K. D.; Griffin, R.

G. Biophys. J. 1988, 54, 373.

(4) Seelig, J.; Waespe-Sarcevic, N. Biochemistry 1978, 17, 3310.

(5) Seelig, J.; Seelig, A. Q. Rev. Biophys. 1980, 13, 19.

(6) Wittebort, R. J.; Blume, A.; Huang, T.; Gupta, S. K. D.; Griffin, R. G. Biochemistry 1982, 21, 3487.

(7) Seelig, J.; Gally, H.-U.; Wohlgemuth, R. Biochim. Biophys. Acta 1977, 467, 109.

(8) Pearson, R. H.; Pascher, I. Nature 1979, 281, 499.

(9) Sanders, C. R. Biophys. J. 1993, 64, 171.

(10) Meier, P.; Ohmes, E.; Kothe, G. J. Chem. Phys. 1986, 85, 3598.

(11) Hong, M.; Schmidt-Rohr, K.; Nanz, D. Biophys. J. 1995a, 69, 1939.

(12) Gally, H.; Niederberger, W.; Seelig, J. Biochemistry 1975, 14, 3647.

(13) Strenk, L. M.; Westerman, P. W.; Doane, J. W. Biophys. J. 1985, 48, 765.

(14) Braach-Maksvytis, V. L. B.; Cornell, B. A. Biophys. J. 1988, 53, 839.

(15) Hong, M.; Schmidt-Rohr, K.; Pines, A. J. Am. Chem. Soc. 1995b, 117, 3310.

(16) Seelig, J. Biochim. Biophys. Acta 1978, 515, 105.

(17) Hauser, H.; Radloff, C.; Ernst, R. R.; Sundell, S.; Pascher, I. J. Am. Chem. Soc. $1988,110,1054$.

(18) Seelig, A.; Seelig, J. Biochim. Biophys. Acta 1975, 406, 1.

(19) Seelig, A.; Seelig, J. Biochemistry 1974, 13, 4839. 


\section{CHAPTER 7}

\section{DETERMINATION OF C-H DIPOLAR COUPLINGS IN LIQUID CRYSTALS}

\subsection{Introduction}

The physical properties of thermotropic liquid crystals are strongly dependent on their molecular shapes, a general feature of which is the presence of a rigid (often aromatic) core and a flexible (aliphatic) part. ${ }^{1}$ Correlations between conformational flexibility and orientational order in these molecules have been investigated by various NMR techniques exploiting anisotropic nuclear spin interactions. ${ }^{2}$ Based on these studies, insight into the structure-order relation has been obtained and used for testing molecular models for the nematic liquid crystals. ${ }^{3-6}$ In NMR experiments, the dipolar interaction between the magnetic moments of two nuclei such as ${ }^{13} \mathrm{C}$ and ${ }^{1} \mathrm{H}$ provides a particularly sensitive probe of the segmental order and conformation of these mobile molecules.

Two-dimensional separated local field (SLF) spectroscopy 7.8 has been applied in conjunction with variable-angle spinning ${ }^{9-12}$ to determine $\mathrm{C}-\mathrm{H}$ dipolar couplings in nematic liquid crystals. As discussed in chapter 2, the SLF technique probes the $\mathrm{C}-\mathrm{H}$ dipolar local fields of the ${ }^{13} \mathrm{C}$ spins. It gives rise to $2 \mathrm{D}$ spectra in which the dipolar coupling patterns are separated according to the ${ }^{13} \mathrm{C}$ chemical shifts. However, due to the large number of protons coupled to each carbon, the C-H SLF spectra are intrinsically complex, so it is difficult to extract individual $\mathrm{C}-\mathrm{H}$ couplings - especially long-range couplings - quantitatively. Alternatively, proton-detected local field (PDLF) spectroscopy ${ }^{13-16}$ yields high-resolution $\mathrm{C}-\mathrm{H}$ dipolar couplings by probing the dipolar local fields of the ${ }^{1} \mathrm{H}$ spins, as demonstrated for phosphocholine lipids (chapter 5). The simplicity and superior resolution of 2D PDLF spectra potentially allow the weak longrange $\mathrm{C}-\mathrm{H}$ couplings to be measured. Furthermore, due to its dipolar detection scheme (of 
${ }^{1} \mathrm{Hs}$ rather than ${ }^{13} \mathrm{Cs}$ ), the PDLF technique allows the ${ }^{1} \mathrm{H}$ coupling partner in a $\mathrm{C}-\mathrm{H}$ spin pair to be identified by incorporating ${ }^{1} \mathrm{H}$ chemical shift information. Thus the PDLF technique makes it possible to assign the long-range $\mathrm{C}-\mathrm{H}$ couplings unambiguously. By contrast, it is not possible to assign the dipolar couplings with the traditional SLF technique.

Based on these considerations, we further explore PDLF-based multi-dimensional NMR techniques for determining $\mathrm{C}-\mathrm{H}$ dipolar couplings in a nematic liquid crystal. The techniques are demonstrated on 4-pentyl-4'-biphenylcarbonitrile (5CB), a roomtemperature nematic liquid crystal that has been characterized by ${ }^{2} \mathrm{H}$ quadrupolar couplings, ${ }^{17}{ }^{1} \mathrm{H}-1 \mathrm{H}^{18,19}$ and ${ }^{13} \mathrm{C}-1{ }^{1} \mathrm{H}$ dipolar couplings, ${ }^{9}$ and ${ }^{13} \mathrm{C}$ chemical shift anisotropy. ${ }^{20}$ Here we carry out 2D PDLF experiments under the off-magic-angle spinning condition to measure the sizes of both short-range (directly-bonded) and longrange (non directly-bonded) $\mathrm{C}-\mathrm{H}$ dipolar couplings in 5CB. The short-range couplings are obtained with high precision by conducting the experiement at various spinning angles and fitting the resulting spectral splittings. The long-range dipolar couplings are assigned qualitatively by a ${ }^{13} \mathrm{C} /{ }^{1} \mathrm{H}$ chemical shift correlation experiment ${ }^{21,22}$ involving cross polarization and a ${ }^{1} \mathrm{H}$ spin diffusion period. Subsequently, the PDLF and the chemical shift correlation pulse sequences are combined into one experiment in order to obtain definite assignments of the long-range $\mathrm{C}-\mathrm{H}$ dipolar couplings. The combined pulse sequence involves three apparent time periods, but can be simplified into a novel reduced3D experiment with only two effective time domains. By these techniques, long-range $C$ $\mathrm{H}$ dipolar couplings between the aromatic and the aliphatic sites are determined for the first time, providing information on the structural correlations between these two ubiquitous components of thermotropic liquid crystals.

The experiments are carried out under fast sample spinning at angles different from the magic angle. ${ }^{9,10}$ The behavior of liquid crystals (LC) under mechanical spinning is more complex than that of solids. The magnitude of dipolar couplings in nematic LCs 
depends on the angle $\beta$ between the symmetry axis (the director) of the phase and the $B_{0}$ field according to $P_{2}(\cos \beta)=\frac{1}{2}\left(3 \cos ^{2} \beta-1\right) .12$ In a static liquid crystal, the director is oriented by the magnetic torque to be either parallel or perpendicular to the magnetic field, depending on the sign of the anisotropy of the magnetic susceptibility, $\Delta \chi$. In a spinning liquid crystal, however, the magnetic torque can be overcome by the viscous torque, causing the $\mathrm{LC}$ direstor to align with the spinning axis. For a nematic $\mathrm{LC}$ with a positive $\Delta \chi$, the director can be oriented along the spinning axis only when the spinning angle $\theta$ is smaller than the magic angle $54.7^{\circ} .{ }^{11}$ Consequently, the dipolar couplings are scaled by a factor $0 \leq P_{2}(\cos \theta) \leq 1$, and milder rf fields can be used for both homonuclear and heteronuclear dipolar decoupling.

\subsection{Experimental Conditions}

The liquid crystal 4-pentyl-4'-biphenylcarbonitrile (5CB) was obtained from Aldrich Chemicals (Milwaukee,WI). The sample was placed in a $7 \mathrm{~mm}$ Doty (Columbia, SC) silicon nitride rotor, and the caps were glued to the rotor to prevent sample leakage. All NMR spectra were measured on a home-built $300 \mathrm{MHz}$ spectrometer with a Tecmag pulse programmer and acquisition system. A home-built variable-angle-spinning probehead was used.

The liquid crystal sample was spun at about $1.6 \mathrm{kHz}$ about an axis inclined at an angle $\theta$ to the magnetic field. The spinning angle was controlled to within $\pm 0.1^{\circ}$ by a stepping motor attached to the bottom of the probe and a computerized motor controller. ${ }^{23}$ Prior to each 2D experiment, the spinning angle was referenced to the magic angle. The choice of the spinning angle $\theta$, which determines the scaling factor $P_{2}(\cos \theta)$, was based on two considerations. First, due to the positive anisotropic susceptibility $(\Delta \chi>0)$ of $5 \mathrm{CB}, \theta$ must be smaller than the magic angle in order for the nematic director to align with the rotation axis. ${ }^{11}$ Second, the value of $P_{2}(\cos \theta)$ should optimize the spectral resolution. If $P_{2}(\cos \theta)$ is too small, long-range $\mathrm{C}-\mathrm{H}$ dipolar couplings will hardly be resolved. If it is 
too large, low-power multiple-pulse decoupling may be insufficient to suppress the residual homonuclear couplings and the spectral resolution will deteriorate. In our experiments, spinning angles between $37.9^{\circ}\left(P_{2}=+0.435\right)$ and $52.1^{\circ}\left(P_{2}=+0.067\right)$ were used.

When applying rf decoupling in liquid crystals, caution must be exercised to minimize the rf powers, as the orientational order of these molecules are often sensitive to the temperature gradients. The if fields used in our experiments are between $33 \mathrm{kHz}$ and $38 \mathrm{kHz}$. Typical ${ }^{1} \mathrm{H}$ and ${ }^{13} \mathrm{C} 90^{\circ}$ pulse lengths range from $6.5 \mu$ s to $7.5 \mu \mathrm{s}$. The $\mathrm{CP}$ contact time is $2.5 \mathrm{~ms}$ in most experiments. The if duty cycle for each scan of FID range from $0.5 \%$ to $4 \%$. The cycle time of the MREV-8 multiple-pulse sequence ${ }^{24,25}$ is usually between $110 \mu \mathrm{s}$ and $130 \mu \mathrm{s}$. In the PDLF experiment, a maximum of $360 \mathrm{MREV}-8$ cycles were acquired in the evolution period. This corresponds to an effective evolution time of $20.3 \mathrm{~ms}$ or a dipolar coupling resolution of $16 \mathrm{~Hz}$, assuming the theoretical MREV-8 scaling factor of $0.47 .24,25$ The chemical shift correlation experiment has a maximum evolution time of $256 \mathrm{MREV}-8$ cycles, leading to a ${ }^{1} \mathrm{H}$ chemical shift resolution of $\sim 24 \mathrm{~Hz}$.

The PDLF pulse sequence for measuring the $\mathrm{C}-\mathrm{H}$ dipolar couplings is the same as described in chapters 2 and 5, and will not be elaborated here. The duration of the ${ }^{1} \mathrm{H}$ spinlock pulse before CP is $1 \mathrm{~ms}$. Similar to the PDLF experiments on phosphocholine, only the cosine-modulated dipolar coherence is recorded at the end of the $t_{1}$ period, so the dipolar coupling patterns are symmetrized in the $\omega_{1}$ dimension.

The ${ }^{13} \mathrm{C} /{ }^{1} \mathrm{H}$ chemical shift (CS) correlation spectra were obtained with the pulse sequence of Figure 7.1a. The sequence involves ${ }^{1} \mathrm{H}$ chemical shift evolution, magnetization transfer from ${ }^{1} \mathrm{H}$ to ${ }^{13} \mathrm{C}$ by cross polarization, and ${ }^{13} \mathrm{C}$ chemical shift detection. In the $t_{1}$ period, a MREV-8 multiple-pulse sequence averages out the $\mathrm{H}-\mathrm{H}$ homonuclear couplings, and a ${ }^{13} \mathrm{C} 180^{\circ}$ pulse in the middle of $t_{1}$ refocuses the $\mathrm{C}-\mathrm{H}$ couplings. At the end of the $t_{1}$ period, both cosine- and sine-modulated ${ }^{1} \mathrm{H}$ chemical shift frequencies are recorded using the States method ${ }^{26}$ in order to obtain pure phase ${ }^{1} \mathrm{H}$ 
chemical shift spectra. A $z$-filter before CP not only selects the desired frequency component, but also allows ${ }^{1} \mathrm{H}$ spin diffusion to occur so that long-range $\mathrm{C}-\mathrm{H}$ dipolar couplings can be detected with higher sensitivity.

Due to the States detection scheme ${ }^{26}$ and the MREV-8 effective field direction $(1,0$, 1), a ${ }^{1} \mathrm{H} 45^{\circ}$ pulse $^{27}$ is applied before and after the MREV-8 multiple-pulse sequence (Figure 7.1a), and complex phase cycles are used for the ${ }^{1} \mathrm{H}$ pulses. The tilted MREV-8 effective field causes the trajectory of the ${ }^{1} \mathrm{H}$ magnetization to deviate from the $x y$ plane of the rotating frame, so that the cosine and sine frequencies recorded at the end of $t_{1}$ do not have the same amplitudes. This imbalance leads to a quadrature image at frequency $-\omega$ for a signal occuring at $+\omega$. To remove this quadrature image, a $45^{\circ} y$ pulse is applied at the end of the evolution period and before the $z$-filter, since the pulse effectively rotates the precession plane normal to the MREV-8 effective field axis to the $x y$ plane of the rotating frame. However, the length of the $45^{\circ}$ pulse is difficult to determine precisely, so the practical direction of the MREV-8 effective field may deviate from the theoretical prediction. As a result, a fraction of the quadrature image might still remain. To eliminate the remaining artifact, one can fully phase-cycle the ${ }^{1} \mathrm{H}$ excitation pulse, the mixing pulse preceding the $z$-filter, and the receiver reference phase in a CYCLOPS fashion. To ensure that the initial ${ }^{1} \mathrm{H}$ magnetization created by the fully phase-cycled excitation pulse is always perpendicular to the MREV- 8 effective field, another $45^{\circ}$ pulse along $-y$ is necessary at the beginning of the $t_{1}$ period.

The combined PDLF-HETCOR (i.e. reduced-3D) experiment was carried out with the pulse sequence of Figure 7.1b. In the first time period, ${ }^{1} \mathrm{H}$ magnetization evolves under the $\mathrm{C}-\mathrm{H}$ couplings as in the normal PDLF experiment. A $90^{\circ}$ pulse stores the cosine-modulated dipolar coherence along the $z$-axis for $1 \mathrm{~ms}$ or less. Then the ${ }^{1} \mathrm{H}$ magnetization is flipped back to the $x y$ plane and evolves under the chemical shift interaction in the second time period. The $z$-filter between the PDLF and the chemical shift periods enables the ${ }^{1} \mathrm{H}$ magnetization to be fully phase-cycled for the chemical shift 
evolution. After the ${ }^{1} \mathrm{H}$ chemical shift evolution, a second $z$-filter is applied to facilitate ${ }^{1} \mathrm{H}$ spin diffusion. Finally, the ${ }^{1} \mathrm{H}$ magnetization is transferred to ${ }^{13} \mathrm{C}$ spins by $\mathrm{CP}$, and ${ }^{13} \mathrm{C}$ chemical shifts are observed during the third, detection period.

(a)

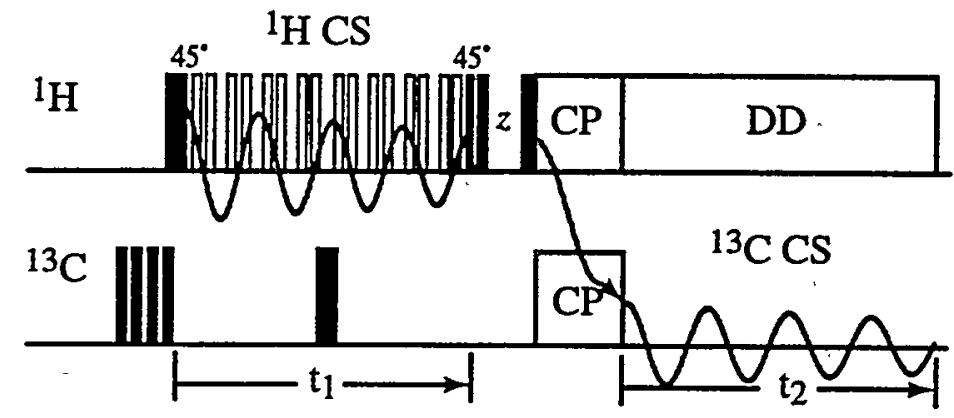

(b)

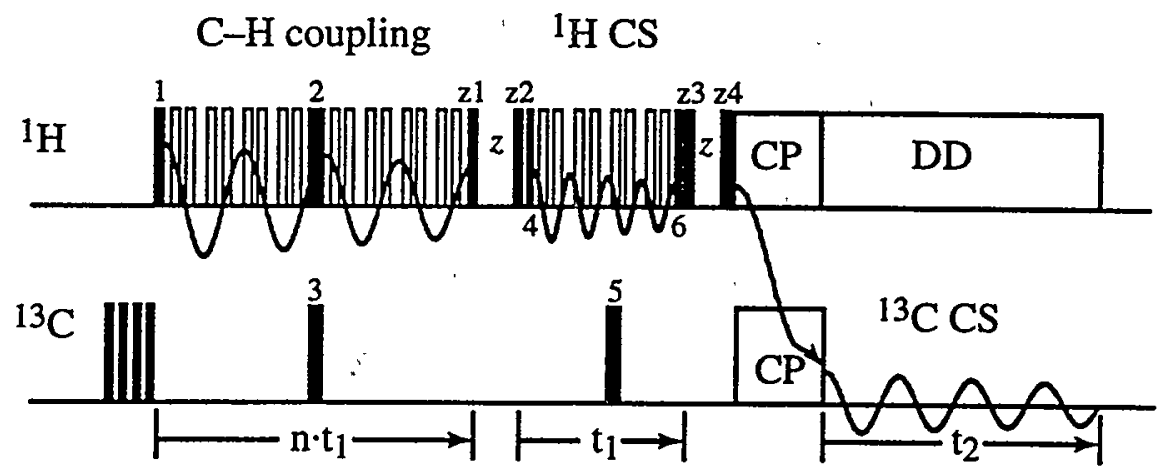

Figure 7.1 (a) HETCOR and (b) reduced-3D pulse sequences. The States method is used to obtain pure-phase ${ }^{1} \mathrm{H}$ chemical shift spectra in both experiments. The PDLF and ${ }^{1} \mathrm{H}$ chemical shift evolutions in (b) are incremented simultaneously to reduce the experimental dimensionality to 2 .

This pulse sequence has three distinct interaction periods, and would normally give rise to a 3D spectrum after successive Fourier transformations. Since a 3D experiment is instrumentally demanding and time-consuming to implement, the pulse sequence is simplified to an effective 2D experiment by a method reminiscent to the "accordion" experiment. ${ }^{28,29}$ Namely, the first two time periods, dominated by the $\mathrm{C}-\mathrm{H}$ couplings and the ${ }^{1} \mathrm{H}$ chemical shifts, are incremented simultaneously so that they give rise to a single frequency dimension after two Fourier transformations. The sizes of increment for the two 
evolution periods need not be the same: as the ${ }^{1} \mathrm{H}$ chemical shift FID decays faster than the dipolar FID, the number of MREV-8 cycles in the chemical shift increment can be smaller than that in the dipolar coupling increment. As a result, in the combined PDLF and ${ }^{1} \mathrm{H}$ chemical shift dimension, the spectral widths (or dwell times) of the two subdimensions differ by a ratio $n$. As will be shown later, the variability of the relative dwell times makes it possible to enhance preferentially the resolution associated with one of the two interactions.

The detailed phase cycles for the reduced-3D pulse sequence are listed below in Table 7.1.

\begin{tabular}{|c|c|c|c|c|c|c|c|c|c|c|c|c|}
\hline pulse & 1 & 2 & 3,5 & $\mathrm{z} 1$ & $\mathrm{z} 2$ & 4 & 6 & $\mathrm{z} 3$ & $\mathrm{z} 4$ & $\mathrm{CPH}$ & $\mathrm{CPC}$ & $\mathrm{RC}$ \\
\hline \multirow{8}{*}{ phase } & 0 & 1 & 0 & $\rho$ & 0 & 3 & 1 & 0 & 2 & 1 & $\begin{array}{ll}0 & 1\end{array}$ & $-\rho$ \\
\hline & 2 & & 0 & $\rho$ & 2 & & & 0 & 2 & 1 & $\begin{array}{ll}0 & 1\end{array}$ & $\rho$ \\
\hline & & & 1 & $\rho$ & 1 & & & 1 & 3 & 2 & 01 & $-\lambda$ \\
\hline & & & 1 & $\rho$ & 3 & & & 1 & 3 & 2 & $\begin{array}{lll}0 & 1\end{array}$ & $\lambda$ \\
\hline & & & 2 & $-\rho$ & & & & 2 & 0 & & 23 & $\rho$ \\
\hline & & & 2 & $-\rho$ & & & & 2 & 0 & & 23 & $-\rho$ \\
\hline & & & 3 & $-\rho$ & & & & 3 & 1 & & 23 & $\lambda$ \\
\hline & & & 3 & $-\rho$ & & & & 3 & 1 & & 23 & $-\lambda$ \\
\hline
\end{tabular}

Table 7.1 Phase cycles for the reduced-3D experiment. $p=2020,-\rho=0202, \lambda=$ $3131,-\lambda=1313, \mathrm{CPH}={ }^{1} \mathrm{H} \mathrm{CP}$ spin lock, $\mathrm{CPC}={ }^{13} \mathrm{C} \mathrm{CP}$ spin lock, $\mathrm{RC}=$ receiver. $z 1-z 4=$ mixing pulses for the two $z$-filters. The $z 3$ pulse phases shown here record the cosine component of the ${ }^{1} \mathrm{H}$ chemical shifts. The sine component is recorded by shifting the $\mathrm{z} 3$ phases by $90^{\circ}$. The pulse nomenclature is indicated in Figure 7.1b.

In all experiments, the ${ }^{1} \mathrm{H}$ carrier frequency is constant and within the ${ }^{1} \mathrm{H}$ spectral region, because the decoupling efficiency of the MREV- 8 multiple-pulse sequence varies with the frequency offset. Improper setting of the ${ }^{1} \mathrm{H}$ carrier frequency could generate artificial splittings in the dipolar dimension of the 2D spectrum. This possibility was eliminated by optimizing the MREV-8 performance with control experiments, whose pulse 
sequence is similar to the PDLF sequence except that the ${ }^{1} \mathrm{H} 180^{\circ}$ pulse in the middle of $t_{1}$ is removed. As a result, the $t_{1}$ period is devoid of any effects of local NMR interactions, and the control spectra should exhibit slow- and smoothly-decaying FIDs in $t_{1}$.

\subsection{Measurement of C-H Dipolar Couplings}

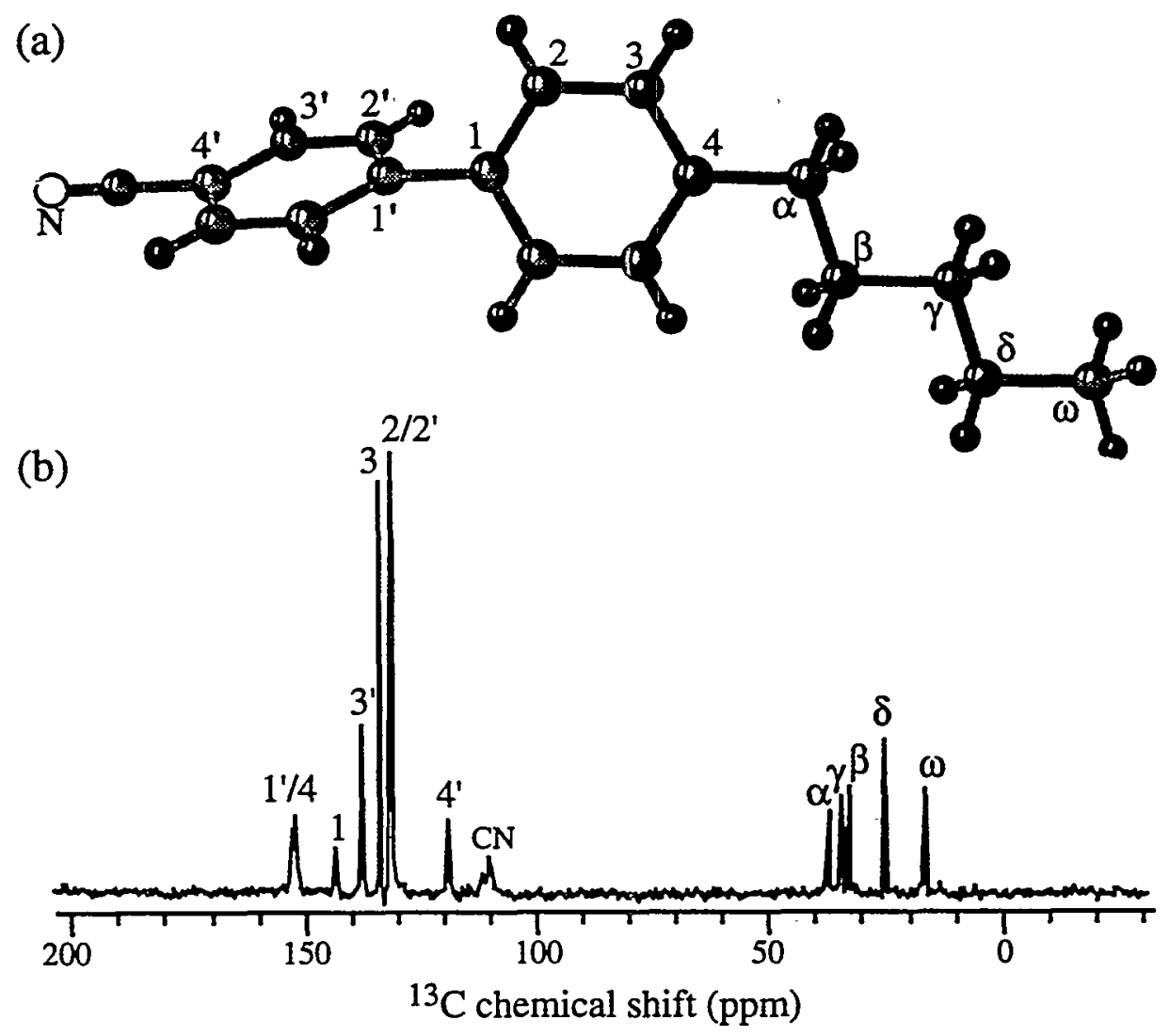

Figure 7.2 4-pentyl-4'-biphenylcarbonitrile (5CB). (a) Chemical structure. (b) ${ }^{13} \mathrm{C}$ CP spectrum under OMAS at $50.7^{\circ}$.

The liquid crystal 5CB consists of an $n$-pentyl chain attached to two aromatic rings, which are further connected to a nitrile group (Figure 7.2a). The ${ }^{13} \mathrm{C}$ chemical shifts of most sites are well resolved and assigned (Figure 7.2b) at OMAS spinning angles of $48^{\circ}-$ $51^{\circ} \cdot{ }^{10}$ 
PDLF spectra of $5 \mathrm{CB}$, correlating the $\mathrm{C}-\mathrm{H}$ couplings with the anisotropic ${ }^{13} \mathrm{C}$ chemical shifts, are obtained at five different OMAS angles $\theta .52 .1^{\circ}, 50.7^{\circ}, 48.2^{\circ}, 42.1^{\circ}$ and $37.9^{\circ}$. Figure 7.3 shows the PDLF spectrum at $\theta=50.7^{\circ}$, corresponding to a scaling factor of $P_{2}=+0.102$. In the dipolar dimension, one or more doublet splittings symmetric about the zero frequency are resolved in each ${ }^{13} \mathrm{C}$ cross section. Since the frequency scale of the $\mathrm{C}-\mathrm{H}$ coupling dimension is corrected for the MREV-8 scaling factor but not for the spinning scaling factor, the spectral splittings correspond, to a first approximation, to the motionally-averaged $\mathrm{C}-\mathrm{H}$ dipolar couplings scaled by the spinning factor $P_{2}\left(\cos 50.7^{\circ}\right)=+0.102$. The largest splitting in each ${ }^{13} \mathrm{C}$ slice resulis from the directlybonded $\mathrm{C}-\mathrm{H}$ coupling. In the aliphatic region (Figure 7.3a), these directly-bonded $\mathrm{C}-\mathrm{H}$ couplings increase from the $\omega-\mathrm{CH}_{3}$ group at the chain end to the $\alpha-\mathrm{CH}_{2}$ group closest to the phenylene rings, except for a size reversal between the $\beta$ and $\gamma$ segments. This general increasing trend reflects the increasing stiffness of the chain towards the sterically rigid aromatic sites.

In addition to the large directly-bond $\mathrm{C}-\mathrm{H}$ splittings, smaller dipolar splittings at $\mathrm{C} \delta$ $(69 \pm 17 \mathrm{~Hz}), \mathrm{C} \alpha(69 \pm 17 \mathrm{~Hz})$ and $\mathrm{C} \gamma(35 \pm 17 \mathrm{~Hz})$ sites and a broadened zero-frequency peak at the $C \beta$ site are observed. Similar to but independent of the directly-bonded couplings, these small C-H splittings are symmetric about the zero frequency of the dipolar dimension. They originate from the dipolar couplings between unbonded ${ }^{13} \mathrm{C}$ and ${ }^{1} \mathrm{H}$ spins, and can be assigned tentatively based on geometric arguments. For example, the small $\mathrm{C}-\mathrm{H}$ splitting in the $\mathrm{C} \delta$ cross section reflects either the $\mathrm{C} \delta-\mathrm{H} \omega$ coupling or the $\mathrm{C} \delta$ $\mathrm{H} \gamma$ coupling. Although no secondary splitting is resolved in the $\mathrm{C} \omega$ slice, the zerofrequency peak results from the unresolved long-range $\mathrm{C}-\mathrm{H}$ couplings. This zerofrequency peak is relatively sharp and has low intensity compared to the broader and more intense zero-frequency peaks in other carbon slices. This is probably due to the additional motional averaging by the three-site jumps of the $\mathrm{C} \omega$ methyl group. Recall that, in comparison to the PDLF spectrum, the zero-frequency region of a $\left({ }^{13} \mathrm{C}\right.$-detected) SLF 


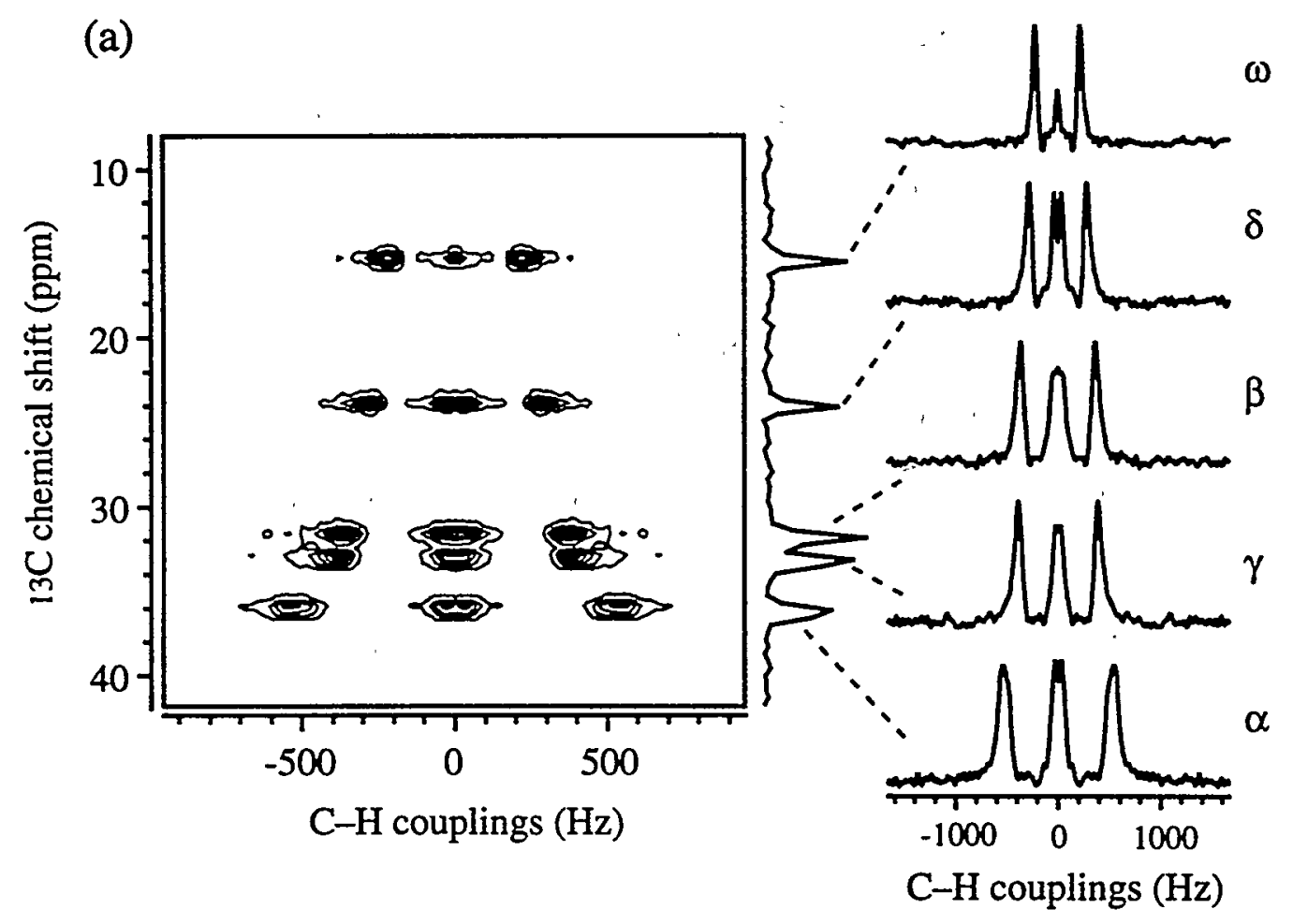

Figure 7.3 5CB PDLF spectrum. (a) Aliphatic region. Long-range C-H dipolar couplings are resolved at $\mathrm{C} \delta, \mathrm{C} \gamma$ and $\mathrm{C} \alpha$ sites. (b) Aromatic region. $\mathrm{C} 2 / \mathrm{C} 2$ ', $\mathrm{C} 3$, and C3' sites exhibit double splittings, and quaternary $\mathrm{C} 4$ and $\mathrm{C}^{\prime}$ ' carbons show long-range couplings. OMAS spinning angle: $50.7^{\circ}$. Measuring time: 15 hours. 


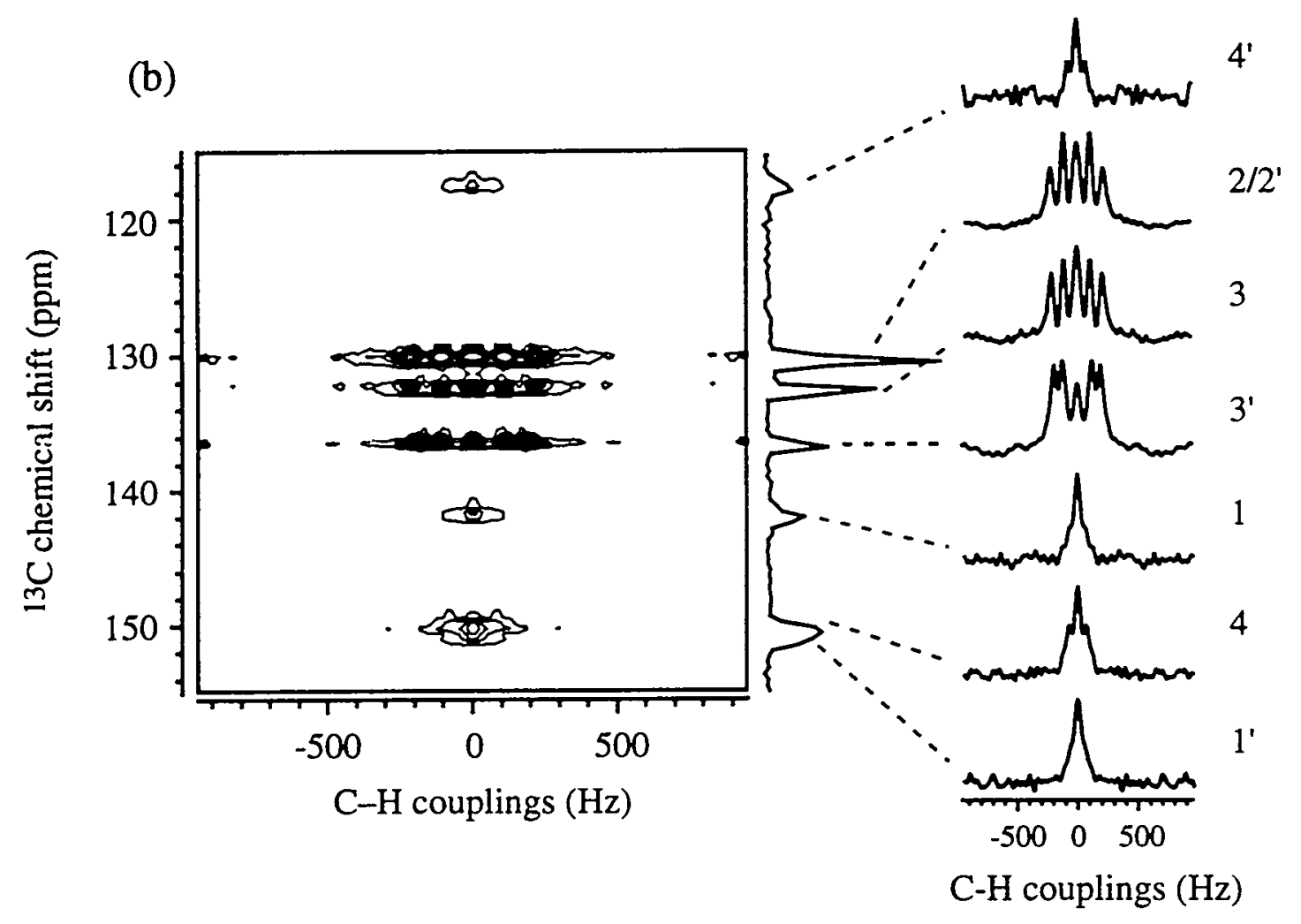


spectrum is crowded with overlapping signals from both long-range dipolar couplings and difference frequencies of near-degenerate directly-bonded dipolar couplings. Thus the SLF spectra should display lower resolution than the PDLF spectra in the center of the dipolar dimension.

An exact analysis of the dipolar splittings in the above PDLF spectrum must account for the fact that the directly-bonded $\mathrm{C}-\mathrm{H}$ splittings also have contributions from the C-H scalar $(J)$ coupling. Since the $J$ coupling is isotropic and does not change with the spinning scaling factor $P_{2}(\cos \theta)$, the contribution of the $J$ coupling can be separated from that of the dipolar coupling by comparing PDLF spectra measured at different spinning angles. ${ }^{10,30}$ This approach has been used to determine the signs and magnitudes of $\mathrm{C}-\mathrm{H}$ dipolar couplings in phosphocholine lipids (chapter 5). However, the spinning angles for the LC sample are limited to values smaller than the magic angle, because the positive $\Delta \chi$ of $5 \mathrm{CB}$ dictates that the nematic director aligns with the spinning axis only when $\theta<$ $54.7^{\circ} .{ }^{12}$ Consequently, the signs of the $\mathrm{C}-\mathrm{H}$ dipolar couplings in $5 \mathrm{CB}$ cannot be obtained. On the other hand, the magnitudes of these dipolar couplings can be determined with high precision by linear regression of several $\mathrm{C}-\mathrm{H}$ splittings obtained with various spinning scaling factors. The strengths of the dipolar couplings, which scale with $P_{2}(\cos \theta)$, correspond to the slope of the linear fit and the $J$ coupling as the intercept. The magnitudes of the directly-bonded $\mathrm{C}-\mathrm{H}$ couplings determined by this method are shown in Figure 7.4. Plotted against the spinning scaling factors are the oscillation frequencies of the dipolar couplings, which are defined as half of the splittings in the spectra. The oscillation frequencies are used instead of splittings, as they allow us to compare our PDLF results consistently with SLF results from the literature. ${ }^{10}$

The results of linear fitting for the directly-bonded $\mathrm{C}-\mathrm{H}$ splittings of the aliphatic sites are displayed in Figure 7.4a. The slopes of the equations correspond to the magnitudes of the directly-bonded $\mathrm{C}-\mathrm{H}$ dipolar couplings, which are summarized in Table 7.2. The fittings are generally quite good $\left(\mathrm{R}^{2}>0.99\right)$. Compared to the SLF-derived $\mathrm{C}-\mathrm{H}$ 
couplings, the PDLF dipolar couplings are $7-22 \%$ smaller (Table 7.2). This deviation may be attributed to differences in sample temperatures and preparation. The precision of the PDLF couplings will be discussed in detail later. It is important to note that the OMASPDLF results described here are consistent with the results of a static PDLF experiment, whose scaling factor for the dipolar couplings is 1.0 .
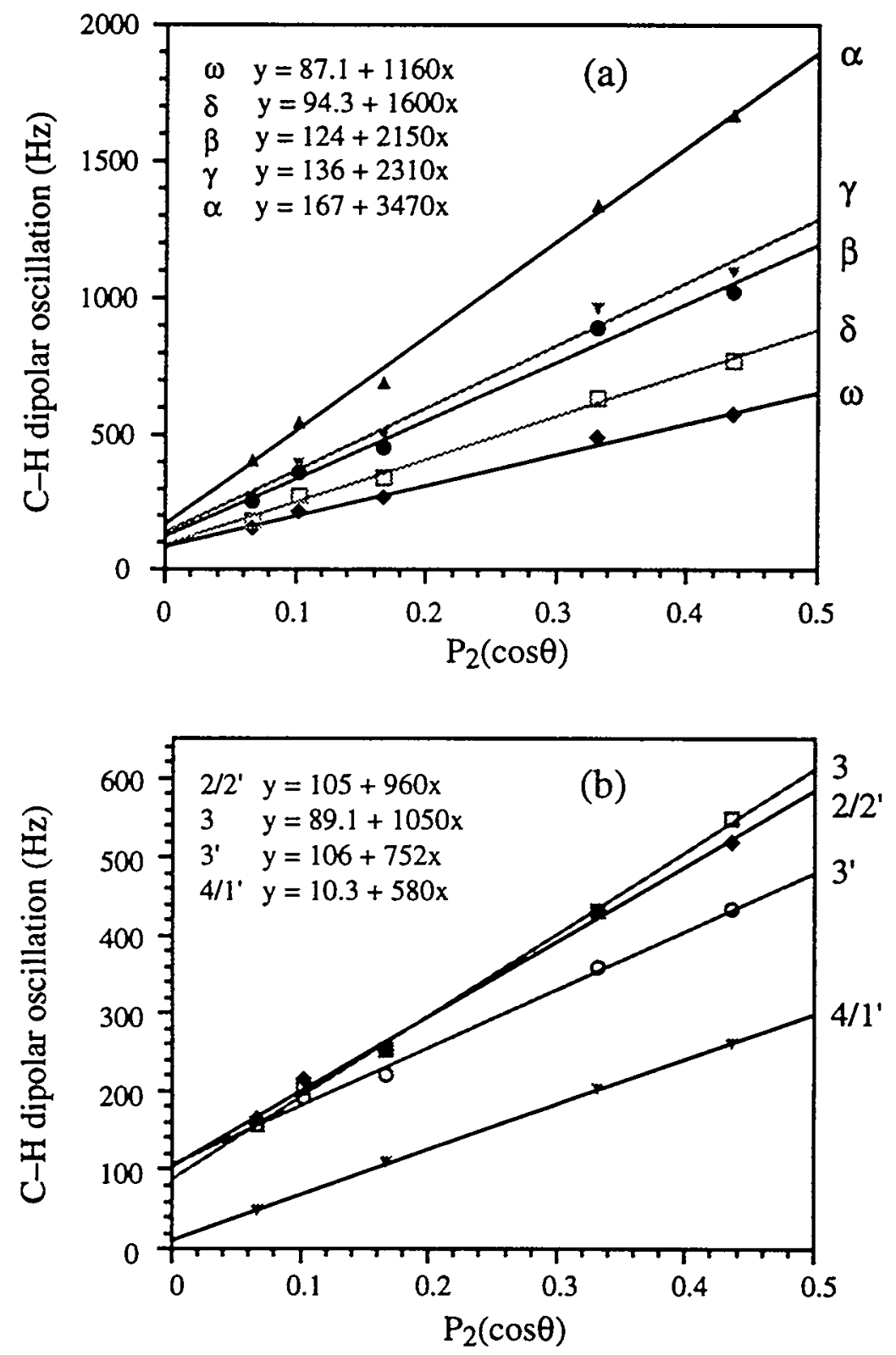

Figure 7.4 Directly-bonded C-H dipolar couplings in 5CB. They are obtained from five PDLF experiments at five different OMAS angles. 
While the short-range one-bond $\mathrm{C}-\mathrm{H}$ dipolar couplings have been measured before by SLF experiments, the long-range $\mathrm{C}-\mathrm{H}$ dipolar couplings are determined here for the first time. The approximate strengths of these couplings can be read off directly from the splittings observed in the spectra. Adjustments for small ${ }^{n} J_{C-H}(n>1)$ couplings are not made here, because the multiple bond $\mathrm{C}-\mathrm{H} J$ couplings are relatively small, ${ }^{31}$ and the signs of the $\mathrm{C}-\mathrm{H}$ dipolar couplings relative to the $J$ coupling, which are required for taking into account the $J$ coupling in the observed splitting, are not determined from these PDLF spectra.

The $\mathrm{C}-\mathrm{H}$ dipolar couplings of the aromatic sites are shown in Figure $7.3 \mathrm{~b}$. The directly-bonded $\mathrm{C}-\mathrm{H}$ couplings are generally smaller than the aliphatic ones, probably because the phenylene $\mathrm{C}-\mathrm{H}$ bonds are oriented nearer the magic angle with the nematic axis. The most significant features in the spectrum are the double splittings of the protonated $\mathrm{C} 2 / \mathrm{C}^{\prime}, \mathrm{C} 3$, and $\mathrm{C} 3{ }^{\prime}$ sites, where the smaller splitting $(-230 \mathrm{~Hz})$ is about half the size of the larger splitting. Since each protonated ${ }^{13} \mathrm{C}$ has only one directly-bonded proton, the smaller splittings must result from the long-range $\mathrm{C}-\mathrm{H}$ couplings. Based on geometric arguments, these long-range couplings can be assigned tentatively to the proton of the neighbouring ${ }^{13} \mathrm{C}$ on the same ring. For instance, the small splitting in the $\mathrm{C} 3$ slice can be attributed to the $\mathrm{C} 3-\mathrm{H} 2$ dipolar coupling, and the small splitting in the $\mathrm{C} 2 / \mathrm{C} 2$ ' slice is due to the $\mathrm{C} 2-\mathrm{H} 3$ and $\mathrm{C} 2^{\prime}-\mathrm{H} 3^{\prime}$ couplings. The coincidence of the $\mathrm{C} 2$ and $\mathrm{C} 2{ }^{\prime}$ chemical shifts makes it difficult to distinguish the $\mathrm{C}-\mathrm{H}$ dipolar couplings of these two sites. The short-range $\mathrm{C}-\mathrm{H}$ couplings of different phenylene sites are quite similar, suggesting a conformation in which corresponding $\mathrm{C}-\mathrm{H}$ bonds (e.g. $\mathrm{C} 2-\mathrm{H} 2$ and $\mathrm{C} 2$ '-H2' bonds) form similar angles with the symmetry axis of the nematic phase.

The high resoluton of the PDLF technique is further demonstrated in the C-H spectra of the quaternary carbons, $\mathrm{C} 4$ and $\mathrm{C} 4$ '. Both sites exhibit a long-range $\mathrm{C}-\mathrm{H}$ coupling of $140 \pm 20 \mathrm{~Hz}$, which are observed for the first time. The splitting in the C4 cross 
section can be assigned to either the intra-aromatic $\mathrm{C} 4-\mathrm{H} 3$ coupling or the aromaticaliphatic $\mathrm{C} 4-\mathrm{H} \alpha$ coupling, since the $\mathrm{C} 4$ carbon is located at the junction of the phenylene ring and the aliphatic chain. In contrast, the splitting in the C4' slice can only be the H3'$\mathrm{C} 4$ ' coupling because the $\mathrm{H} 3^{\prime}$ protons are closest to the quaternary $\mathrm{C} 4$ '.

Two-bond $\mathrm{C}-\mathrm{H}$ dipolar couplings in the protonated aromatic sites have been measured with relatively low resolution by the SLF technique ${ }^{10}$ and refined by simulations. The long-range couplings from the current PDLF experiments (Table 7.3) show qualitative agreements with the SLF results. This confirms the precision of the PDLF method. However, the PDLF exneriment has the advantage that the long-range dipolar couplings can be read off directly from individual splittings in the spectra rather than calculated by spectral simulations. Further, the PDLF technique is able to resolve the dipolar couplings between the quaternary carbons and protons several bonds away, which are not available by the SLF approach.

\begin{tabular}{lllllllll}
\hline sites & $\omega$ & $\delta$ & $\beta$ & $\gamma$ & $\alpha$ & $2 / 2^{\prime}$ & 3 & $3^{\prime}$ \\
\hline PDLF & 1.16 & 1.60 & 2.15 & 2.31 & 3.47 & 0.96 & 1.05 & 0.75 \\
SLF & 1.39 & 1.98 & 2.75 & 2.96 & 4.13 & 1.03 & 1.02 & 0.81 \\
\hline
\end{tabular}

Table 7.2 Directly-bonded C-H dipolar couplings in 5CB. Only absolute values $(\mathrm{kHz})$ are given, as the signs cannot be determined from the experiments described. The SLF results are obtained from the literature. ${ }^{10}$ The error margins are discussed in the text.

In the following, the experiments that allow quantitative assignment and further refinement of the long-range $\mathrm{C}-\mathrm{H}$ couplings are described. The directly-bonded $\mathrm{C}-\mathrm{H}$ dipolar couplings determined in the PDLF spectra are then analyzed in terms of order parameters.

\subsection{Qualitative Assignment of Long-Range C-H Couplings}


In the PDLF spectra, the long-range $\mathrm{C}-\mathrm{H}$ dipolar couplings are assigned tentatively based on distance arguments. This assignment can be corroborated by a ${ }^{13} \mathrm{C} /{ }^{1} \mathrm{H}$ HETCOR experiment that correlates the ${ }^{13} \mathrm{C}$ and ${ }^{1} \mathrm{H}$ chemical shifts. Since the ${ }^{13} \mathrm{C}$ magnetization is transferred from ${ }^{1} \mathrm{H}$ by cross polarization, each peak in the 2D spectrum indicates a specific $\mathrm{C}-\mathrm{H}$ dipolar coupling. Figure 7.5 displays the 5CB HETCOR spectrum obtained with the OMAS angle of $50.7^{\circ}$. The mixing time for ${ }^{1} \mathrm{H}$ spin diffusion is $3 \mathrm{~ms}$ and the $\mathrm{CP}$ contact time is $2.5 \mathrm{~ms}$. In the aliphatic ${ }^{13} \mathrm{C}$ chemical shift region (Figure 7.5a), the ${ }^{1} \mathrm{H}$ chemical shifts of $H \beta, H \gamma$ and $H \delta$ are essentially indistinguishable, whereas the $H \omega$ and the $H \alpha$ chemical shifts are clearly separated from the rest. In addition to resonances from the directly-bonded $\mathrm{C}-\mathrm{H}$ spin pairs, cross peaks due to the unbonded $\mathrm{C}-\mathrm{H}$ pairs such as $\mathrm{C} \beta-$ $\mathrm{H} \alpha, \mathrm{C} \gamma-\mathrm{H} \alpha, \mathrm{C} \gamma-\mathrm{H} \beta$ and $\mathrm{C} \alpha-\mathrm{H} \beta$ are observed at the respective ${ }^{13} \mathrm{C}$ and ${ }^{1} \mathrm{H}$ chemical shifts. Furthermore, aliphatic and aromatic $\mathrm{C}-\mathrm{H}$ cross peaks are observed. For example, in the $\mathrm{C} \alpha$ and $\mathrm{C} \beta$ slices, the spectral intensities above $7.5 \mathrm{ppm}$ result from the $\mathrm{Ci}-\mathrm{H} 3$ and $\mathrm{Ci}-\mathrm{H} 2$ couplings $(\mathrm{i}=\alpha, \beta)$. The possibility that the $\mathrm{H} 2$ ' and $\mathrm{H}^{\prime}$ protons are coupled significantly to these aliphatic carbons can be ruled out based on the large internuclear distances between the aliphatic carbons and the Hi' $(\mathrm{i}=1-4)$ protons as well as the different chemical shifts of the protons on the two different phenyl rings. Judging from the small but discernible chemical shift difference between the H3' and H3 protons, we conclude that the $\mathrm{C} \alpha$ and $\mathrm{C} \beta$ carbons couple to the $\mathrm{H} 2$ and $\mathrm{H} 3$ protons, whose chemical shifts are more upfield than those of the $\mathrm{H} 2$ ' and H3' protons.

The aromatic region of the HETCOR spectrum (Figure 7.5b) confirms the existence of the aliphatic and aromatic $\mathrm{C}-\mathrm{H}$ dipolar couplings. In the $\mathrm{C} 2 / \mathrm{C} 2$ ' and $\mathrm{C} 3$ slices, cross peaks at the $H \alpha$ and $H \beta$ chemical shifts are observed, and the peak intensities are stronger in the $\mathrm{C} 3$ slice than in the $\mathrm{C} 2 / \mathrm{C} 2$ ' slice. This intensity difference is consistent with the closer distance of the $\mathrm{C} 3$ carbon to the aliphatic chain. Furthermore, the quaternary carbon C4 exhibits strong resonances at the $\mathrm{H} 2 / \mathrm{H} 3, \mathrm{H} \alpha$ and $\mathrm{H} \beta$ chemical shifts. This indicates 
(a)

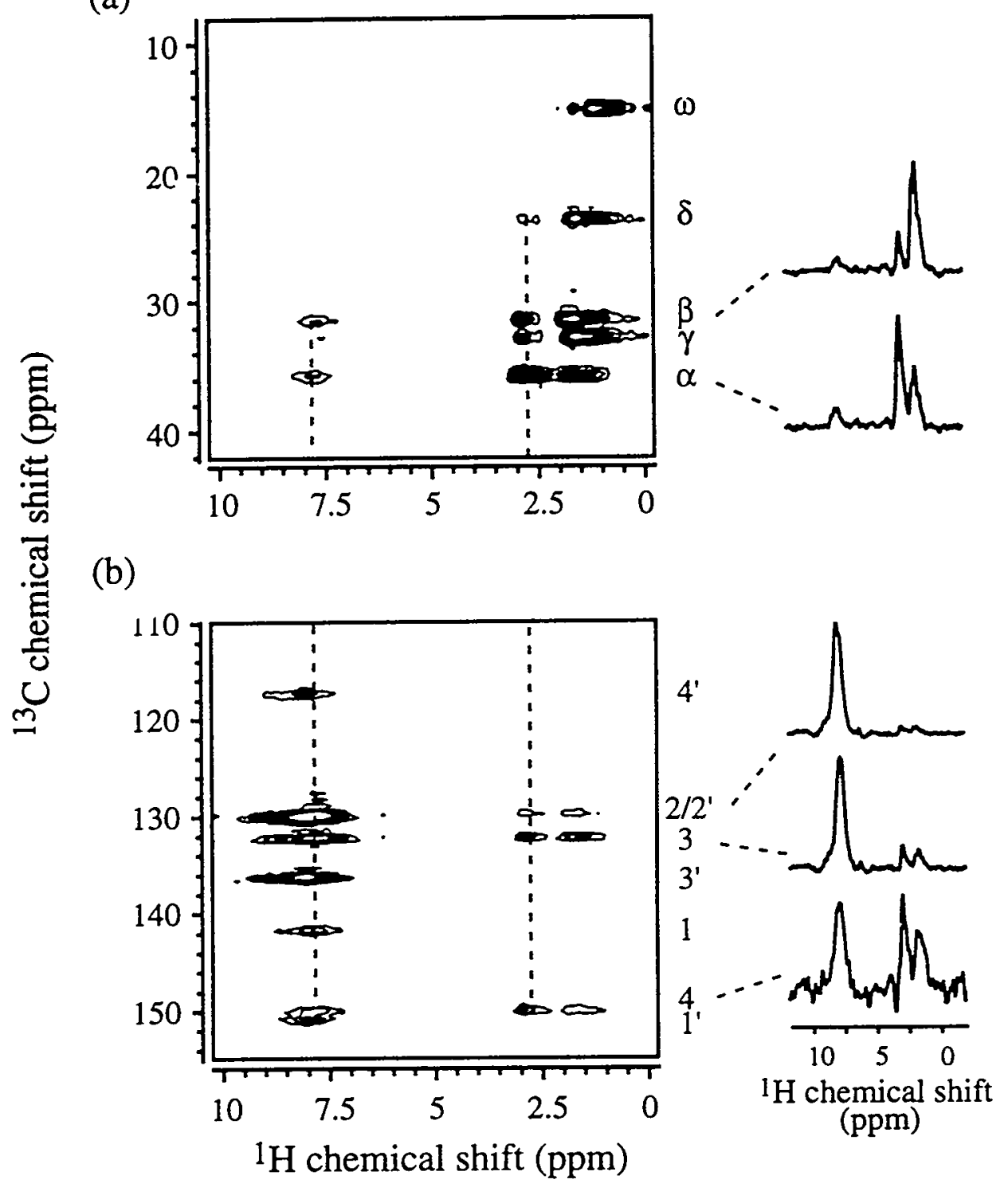

Figure 7.5 $5 \mathrm{CB}{ }^{13} \mathrm{C}^{1} \mathrm{H}$ chemical shift correlation spectrum. (a) Aliphatic region. (b) Aromatic region. Dashed lines guide the eyes for the $\mathrm{H} 3 / 2$ and $\mathrm{H} \alpha$ chemical shifts. Spinning angle: $50.7^{\circ}$. ${ }^{1} \mathrm{H}$ mixing time: $3 \mathrm{~ms}$. CP contact time: $2.5 \mathrm{~ms}$. Measuring time: 18 hours. 
significant $\mathrm{C} 4-\mathrm{H} \alpha$ and $\mathrm{C} 4-\mathrm{H} \beta$ dipolar couplings, which cannot be observed otherwise in the spectrum. In comparison, the quaternary $\mathrm{Cl}, \mathrm{C} 1^{\prime}$ and $\mathrm{C}^{\prime}$ carbons, which are farther away from the aliphatic chain, exhibit resonances only at the chemical shifts of the phenylene ring protons.

\subsection{Quantitative Assignment of Long-Range $\mathrm{C}-\mathrm{H}$ Couplings}

So far, the long-range $\mathrm{C}-\mathrm{H}$ dipolar couplings are resolved by the PDLF technique and qualitatively assigned by the HETCOR experiment. It would be natural to combine these two experiments into a single experiment that correlates the $\mathrm{C}-\mathrm{H}$ dipolar couplings, the ${ }^{1} \mathrm{H}$ chemical shifts and the ${ }^{13} \mathrm{C}$ chemical shifts simultaneously. Such a $3 \mathrm{D}$ experiment would require processing and storage of large data sets, since both the PDLF and the ${ }^{13} \mathrm{C}$ chemical shift dimensions require long acquisition times in cirder to fully resolve the small dipolar couplings and sharp resonance lines typical of motionally-averaged systems. The difficulties of this 3D experiment can be circumvented using the reduced-3D pulse sequence described above (Figure 7.1b).

The reduced-3D spectrum of 5CB acquired with a ${ }^{1} \mathrm{H}$ PDLF:CS dwell time ratio $(n)$ of 4 is displayed in Figure 7.6. The frequency scale of the horizontal axis refers to the dipolar couplings, so the chemical shift frequencies are four times larger. The zero frequency of the axis corresponds to the ${ }^{1} \mathrm{H}$ carrier frequency and is not referenced to any chemical shift standard. In each ${ }^{13} \mathrm{C}$ cross section, several $\mathrm{C}-\mathrm{H}$ doublet splittings displaced according to the ${ }^{1} \mathrm{H}$ chemical shifts can be observed. The center of the largest splitting corresponds to the chemical shift of the proton directly bonded to the carbon of interest, whereas the centers of the smaller splittings correspond to the chemical shifts of the unbonded protons that are coupled to the same carbon, thereby revealing the identities of these protons. 
In the aliphatic region (Figure 7.6a), long-range $\mathrm{C} \alpha-\mathrm{H} \beta$ and $\mathrm{C} \alpha-\mathrm{H} 3$ dipolar couplings can be identified, as the center of the directly-bonded $\mathrm{C} \alpha-\mathrm{H} \alpha$ coupling, i.e. the $\mathrm{H} \alpha$ chemical shift, is well displaced from the centers of the long-range $\mathrm{C} \alpha-\mathrm{H}$ splittings. The $\mathrm{C} \alpha-\mathrm{H} \beta$ coupling exhibits as a splitting of $74 \pm 17 \mathrm{~Hz}$ centered at $+342 \mathrm{~Hz}$, which coincides with the center of the $C \beta-H \beta$ doublet in the $C \beta$ cross section. This magnitude of the $\mathrm{C} \alpha-\mathrm{H} \beta$ coupling is consistent with the result of the PDLF spectrum, and confirms the assignment by the HETCOR experiment. In addition, one can identify a peak at $-124 \mathrm{~Hz}$ with an unresolved splitting, which can be assigned to the $\mathrm{C} \alpha-\mathrm{H} 3$ or the $\mathrm{C} \alpha-\mathrm{H} 2$ coupling. A distinction between these two possibilities cannot be made based on this spectrum due to the similarity of the $\mathrm{H} 2$ and $\mathrm{H} 3$ chemical shifts at this specific spinning angle.

Because of the limited resolution of the dipolar couplings in the spectrum, the magnitude of the $\mathrm{C} \alpha-\mathrm{H} 3$ (or $\mathrm{C} \alpha-\mathrm{H} 2$ ) coupling cannot be determined quantitatively. In fact, the mixed dipolar-shift dimension of the reduced-3D spectrum shows less-resolved resonances than the pure dipolar dimension of the PDLF spectrum. The time evolution corresponding to the combined frequency dimension is a product of two decaying signals,

$$
F\left(t_{1}\right)=f\left(\omega_{C S} t_{1}\right) \exp \left(-t_{1} / T_{2 C S}^{*}\right) * f\left(n \omega_{D} t_{1}\right) \exp \left(-n t_{1} / T_{2 D}^{*}\right)
$$

where $T_{2 C S}^{*}$ and $T_{2 D}^{*}$ are the decay constants of the chemical shift and dipolar coupling FIDs, respectively. The Fourier transform of the function (7.1) with respect to $t_{1}$ is the convolution of its two contributions. For the case of an isolated $\mathrm{C}-\mathrm{H}$ spin pair, the spectrum is a doublet centered at the ${ }^{1} \mathrm{H}$ chemical shift and has a FWHM of $\left(1 / T_{2 C S}^{*}+n \cdot 1 / T_{2 D}^{*}\right) / \pi \mathrm{Hz}$. Since the $180^{\circ}$ pulse in the PDLF evolution refocuses field inhomogeneities as well as chemical shifts, $T_{2 D}^{*}>T_{2 C S}^{*}$. Thus the best resolution in the mixed frequency dimension of the reduced-3D spectrum is achieved when the chemical shift lineshape acts as a matched filter for the dipolar signal, i.e. when $T_{2 C S}^{*}=T_{2 D}^{*} / n$. 


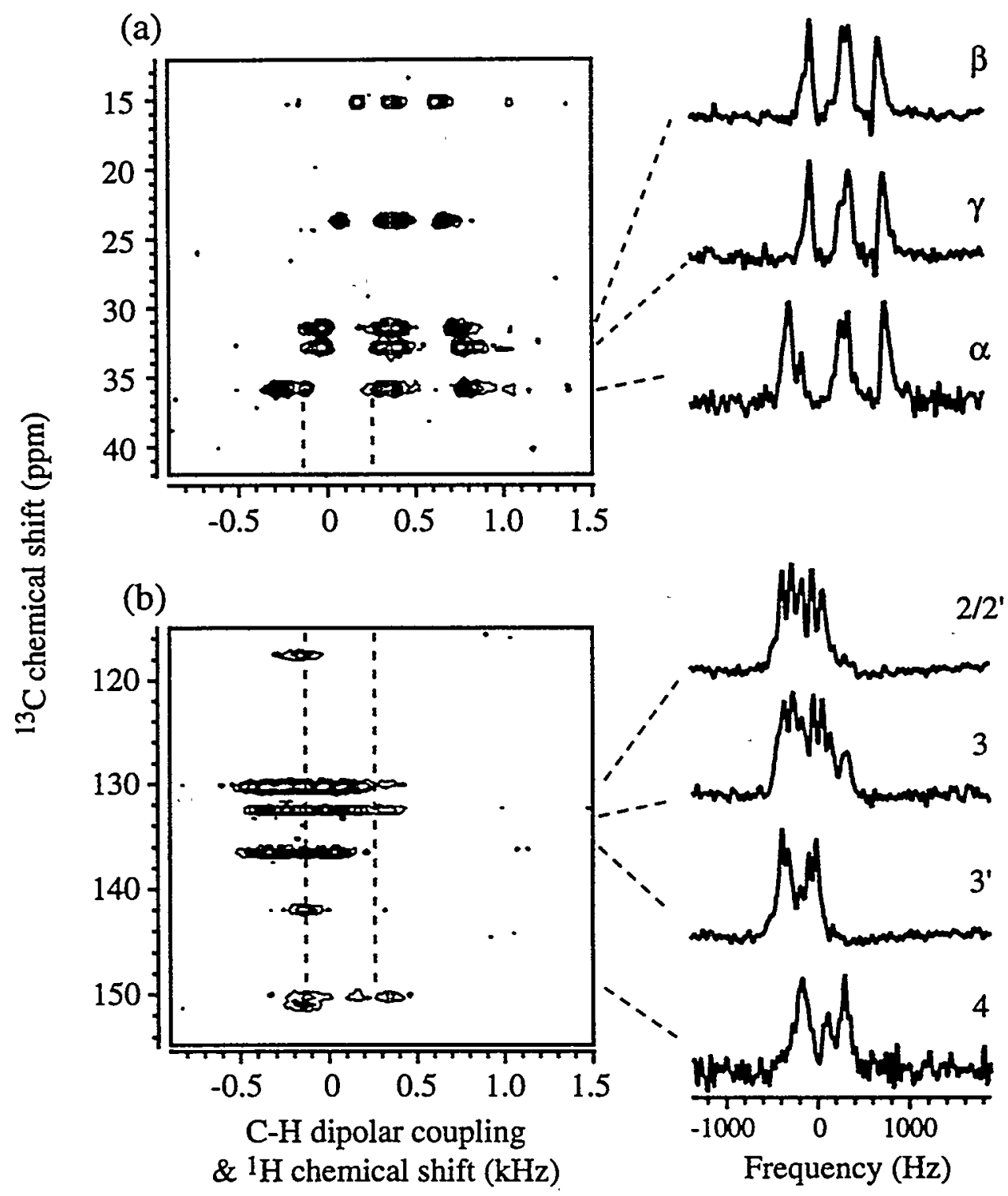

Figure 7.6 $5 \mathrm{CB}$ reduced-3D spectrum with ${ }^{1} \mathrm{H}$ PDLF:CS dwell ratio of 4 . (a) Aliphatic region. (b) Aromatic region. $\mathrm{C}-\mathrm{H}$ dipolar couplings between aromatic carbons and aliphatic protons are observed. Left and right dashed lines correspond to the $H 3 / 2$ and $H \alpha$ chemical shifts. Measuring time: 15.5 hours. 
This effect is demonstrated, for example, by the higher resolution of the $C \beta$ cross section in the spectrum with $n=4$ (Figure 7.6) than in the spectrum with $n=2$ (Figure 7.7).

The lack of resolved long-range dipolar couplings in the PDLF spectrum does not necessarily indicate the resolution limit of the reduced-3D spectrum. In the PDLF spectrum, all $\mathrm{C}-\mathrm{H}$ couplings of a ${ }^{13} \mathrm{C}$ site are centered at the zero frequency, so the zerofrequency peak can be the superposition of several long-range $\mathrm{C}-\mathrm{H}$ couplings. In the reduced-3D spectrum, however, these small long-range dipolar couplings are displaced according to the ${ }^{1} \mathrm{H}$ chemical shifts so that enhanced resolution is possible. The $C \beta$ cross section in Figure 7.3a and Figure 7.6a demonstrates such a resolution difference.

Figure 7.6b shows the aromatic ${ }^{13} \mathrm{C}$ region of the same reduced-3D spectrum $(n=$ 4). One can observe intra-aromatic $\mathrm{C}-\mathrm{H}$ couplings, which are barely shifted from the directly-bonded $\mathrm{C}-\mathrm{H}$ couplings due to the nearly identical chemical shifts of the aromatic protons at this spinning angle. Dipolar couplings between the aliphatic protons and the aromatic carbons can be identified more clearly. For example, the $\mathrm{C} 3-\mathrm{H} \alpha$ coupling can be assigned. The left peak of the splitting is manifested as a shoulder of the main signals at about $173 \mathrm{~Hz}$, while the right peak occurs at $297 \mathrm{~Hz}$. The center of these two frequencies matches the $\mathrm{H} \alpha$ chemical shift. According to this assignment, the $\mathrm{C} 3-\mathrm{H} \alpha$ coupling is determined to be $120 \pm 30 \mathrm{~Hz}$. In comparison, the C3' cross section exhibits no cross peaks in the aliphatic ${ }^{1} \mathrm{H}$ chemical shift region, which is consistent with the larger distance of the $\mathrm{C} 3^{\prime}$ carbon to the aliphatic chain. The $\mathrm{C} 4$ slice exhibits unresolved signals at the chemical shift of the aromatic $\mathrm{H} 2 / \mathrm{H} 3$, as well as an asymmetric doublet at the $\mathrm{H} \alpha$ chemical shift. The magnitude of the $\mathrm{C} 4-\mathrm{H} \alpha$ coupling is $180 \pm 30 \mathrm{~Hz}$, which is consistent with the PDLF result within the error margin. Overall, our spectra indicate consistently that the $\mathrm{C} 4$ carbon couples more strongly to the aliphatic $\mathrm{H} \alpha$ proton than to the aromatic protons.

To further confirm the assignment of the long-range $\mathrm{C}-\mathrm{H}$ dipolar couplings, we performed a second reduced-3D experiment using a ${ }^{1} \mathrm{H}$ PDLF:CS dwell time ratio $(n)$ of 2 , keeping the chemical shift dwell time the same as before. This causes a contraction of the 


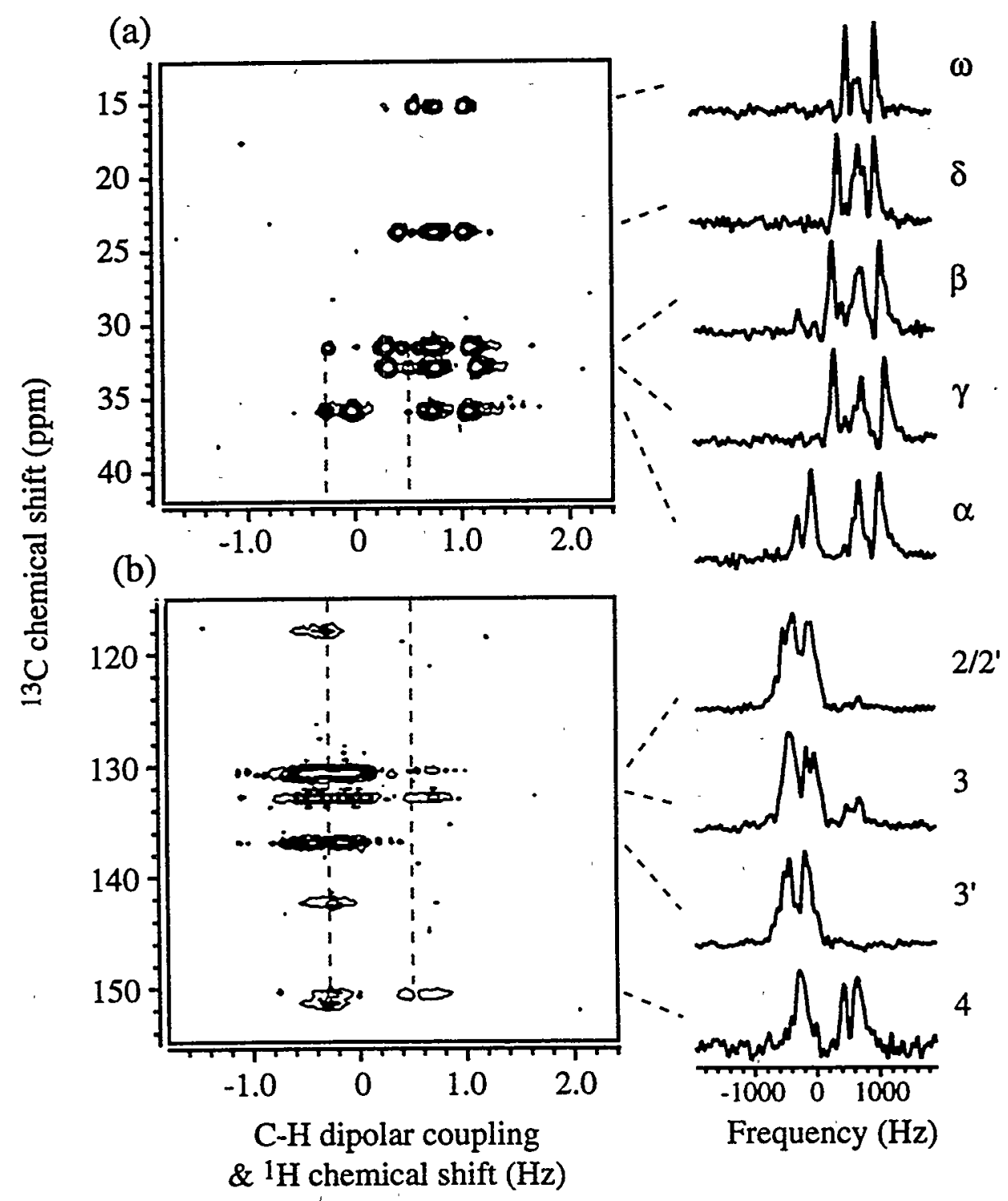

Figure 7.7 5CB reduced-3D spectrum with ${ }^{1} \mathrm{H}$ PDLF:CS dwell ratio of 2 . (a) Aliphatic region. (b) Aromatic region. The long-range $\mathrm{C}-\mathrm{H}$ splittings are more displaced from the centers of the one-bond $\mathrm{C}-\mathrm{H}$ splittings compared to Figure 7.5 due to the reduced ${ }^{1} \mathrm{H}$ chemical shift spectral width. Left and right dashed lines correspond to the $H 3 / 2$ and $H \alpha$ chemical shifts. Measuring time: 19 hours. 
spectral window of the ${ }^{1} \mathrm{H}$ chemical shift relative to the dipolar coupling, and results in an increased separation of the $\mathrm{C}-\mathrm{H}$ doublets in each ${ }^{13} \mathrm{C}$ cross section. Figure 7.7 exhibits the reduced-3D spectrum with $n=2$. In the $\mathrm{C} \alpha$ slice (Figure 7.7a), the intense signal at 725 $\mathrm{Hz}$ coincides with the chemical shifts of $\mathrm{H} \beta$ or $\mathrm{H} \gamma$ and is clearly shifted from the center of the large doublet with a splitting of $1.08 \mathrm{kHz}$. The dipolar coupling of $\mathrm{C} \alpha$ with the aromatic $\mathrm{H} 3$ or $\mathrm{H} 2$ protons is manifested as an unsplit peak at $-264 \mathrm{~Hz}$. In the aromatic ${ }^{13} \mathrm{C}$ region (Figure $7.7 \mathrm{~b}$ ), the $\mathrm{C} 3$ cross section exhibits a $\mathrm{C} 3-\mathrm{H} \alpha$ dipolar coupling of $100 \pm 25 \mathrm{~Hz}$, consistent with the result of Figure 7.6. Due to the reduced dwell time of the dipolar evolution, the Icsolution of the $\mathrm{C}-\mathrm{H}$ couplings is lower in Figure 7.7 than in the previous spectrum, and many long-range $\mathrm{C}-\mathrm{H}$ dipolar couplings cannot be measured precisely.

Table 7.3 summarizes the long-range $\mathrm{C}-\mathrm{H}$ dipolar couplings in $5 \mathrm{CB}$, measured and assigned by the PDLF, HETCOR, and reduced-3D experiments. The coupling strengths are calculated as the averaged values from the spectra of Figures 7.3, 7.6 and 7.7, and weighted according to the respective resolutions of these spectra. The convention of defining half of the splitting as the dipolar coupling is followed. Since the signs of the couplings cannot be obtained from the current spectra, the absolute magnitudes are given.

\begin{tabular}{c|cccccc}
\hline${ }^{13} \mathrm{C}$ & $\mathrm{C} \delta$ & $\mathrm{C} \gamma$ & $\mathrm{C} \beta$ & $\mathrm{C} \alpha$ & $\mathrm{C} 4$ & \\
${ }^{1} \mathrm{H}$ & $\mathrm{H} \omega$ & $\mathrm{H} \beta$ & $\mathrm{H} \delta / \mathrm{H} \gamma$ & $\mathrm{H} \beta$ & $\mathrm{H} \alpha$ & \\
coupling & $0.36 \pm 0.10$ & $0.17 \pm 0.10$ & $0.30 \pm 0.10$ & $0.35 \pm 0.12$ & $0.76 \pm 0.15$ & \\
\hline${ }^{13} \mathrm{C}$ & $\mathrm{C} 3$ & $\mathrm{C} 3$ & $\mathrm{C} 2$ & $\mathrm{C}^{\prime}$ & $\mathrm{C}^{\prime}$ & $\mathrm{C}^{\prime}$ \\
${ }^{1} \mathrm{H}$ & $\mathrm{H} \alpha$ & $\mathrm{H} 2$ & $\mathrm{H} 3$ & $\mathrm{H}^{\prime}$ & $\mathrm{H}^{\prime}$ & $\mathrm{H}^{\prime}$ \\
coupling & $0.54 \pm 0.15$ & $1.12 \pm 0.10$ & $1.17 \pm 0.10$ & $1.17 \pm 0.10$ & $1.11 \pm 0.10$ & $0.72 \pm 0.10$ \\
\hline
\end{tabular}

Table 7.3 Resolved and assigned long-range $\mathrm{C}-\mathrm{H}$ dipolar couplings in 5CB. Only the absolute values $(\mathrm{kHz})$ are given. 
The resolution of the reduced-3D spectra can be improved by changing the spinning angles to values corresponding to larger scaling factors. A factor of three or four in $P_{2}(\cos \theta)$ could be gained if the rotor axis is inclined at $43^{\circ}$ or $39^{\circ}$ to the magnetic field instead of $51^{\circ}$. Unfortunately, there are drawbacks associated with setting the spinning axis at smaller angles. First, the rf coil in our VAS probehead is wrapped around the stator, ${ }^{23}$ so the effective if power is reduced when the stator axis is aligned more with the magnetic field. As a result, MREV-8 decoupling would become less efficient, resulting in a loss of spectral resolution. In addition, the dipolar Hamiltonian parameters also become larger at spinning angles further away from the magic angle, making homonuclear and heteronuclear dipolar decoupling more difficult. In principle, the highest resolution in dipolar couplings can be achieved by a static PDLF experiment, which has a scaling factor of either 1 or 0.5 depending on whether the nematic director is parallel or perpendicular to the magnetic field direction. On the other hand, the additional degree of freedom due to spinning allows delicate control of the experimental conditions, such as the maximum if power and the largest detectable frequency. The latter is also a function of the pulse length, as the dwell time of the indirect dimension is constrained to be a multiple of the MREV-8 cycle time.

\subsection{Order Parameters from Short-Range C-H Couplings}

The motionally-averaged NMR $\mathrm{C}-\mathrm{H}$ dipolar couplings in $5 \mathrm{CB}$ provides information on the structure and the overall dynamics of this anisotropically mobile molecule. As discussed in chapter 4, the average dipolar coupling between spin $I$ and spin $S$ in a rigid liquid-crystalline molecule can be described by the Saupe order matrix, $S_{i j}$,

$$
\bar{\delta}_{d}=-\frac{\mu_{0}}{4 \pi} \gamma_{I} \gamma_{s} \hbar \frac{1}{r^{3}}\left\langle\frac{1}{2}\left(3 \cos ^{2} \theta-1\right)\right\rangle \propto \frac{1}{r^{3}} \sum_{x, y, z} S_{i j} D_{i j}
$$


In the equation, $r$ is the internuclear distance, $\gamma_{I}$ and $\gamma_{s}$ are the gyromagnetic ratios of the $I$ and $S$ spins, $\theta$ is the angle between the internuclear vector and the magnetic field, and $D_{i j}$ $\propto \frac{1}{r^{3}}\left(3 \cos \theta_{i} \cos \theta_{j}-\delta_{i j}\right)$ are the elements of the dipolar coupling tensor in the same molecule-fixed frame as the order tensor. The order matrix $S_{i j}$ describes the averaged spatial orientation of molecular segments with respect to the nematic director according to equation (4.11).

For molecules with internal degrees of freedom, equation (7.2) is not strictly valid, since the motion could change the internuclear distances, and each aliowed conformer could experience different orientational forces and thus have a different set of order parameters. Dipolar couplings that are sensitive to the internal dynamics, for example between nuclei whose internuclear distances vary signicantly in different conformers, can only be reproduced by a model that takes into account the interdependence between the molecular shape and the anisotropic properties. Such model-dependent analyses have been made about the dynamics of the aliphatic chain in 5CB using NMR constraints. ${ }^{3}$ On the other hand, the phenylene rings in $5 \mathrm{CB}$ can be treated as simple "rigid" subunits, so that the ring dipolar couplings can be analyzed independently. The number of order parameters necessary to describe the ring system can be evaluated based on symmetry arguments. ${ }^{32}$ If a quasi-hexagonal symmetry is assumed for the rings, then only two order parameters, $S_{z z}$ and $S_{x x}-S_{y y}$, are necessary to describe the molecular orientations. (This symmetry assumption is strictly valid only for the ring carrying the aliphatic chain. A small deviation from this symmetry has been calculated for the ring bearing the nitrile group, as the two protons next to the $\mathrm{CN}$ group are slightly tilted back to form a $\mathrm{C}-\mathrm{C}-\mathrm{H}$ bond angle of about $121^{\circ}$.)

Table 7.4 shows the resulting order parameters for the two phenylene rings, calculated from the dipolar couplings from the OMAS PDLF spectra and the static PDLF spectrum. They are compared with the order parameters from the OMAS SLF spectra in 
the literature. Standard bond lengths $\left(r_{\mathrm{C}-\mathrm{H}}=1.09 \AA, r_{\mathrm{C}-\mathrm{C}}=1.40 \AA\right)$ are used in the calculations. The main order parameter $S_{z z}$ is most sensitive to temperature, as it describes the global order of the phase. The difference in the $S_{z z}$ values between the static and the spinning PDLF experiments (about 0.52 and 0.41 , respectively) is attributed to the $4^{\circ}$ room temparature difference between the two laboratories where the experiments were performed. In each data set, the order parameter in the ring carrying the $\mathrm{CN}$ group (ring 2) is found to be slightly larger, as observed previously.

\begin{tabular}{c|cccccccccc}
\hline & \multicolumn{3}{|c}{ static PDLF } & \multicolumn{4}{c}{ OMAS PDLF } & \multicolumn{3}{c}{ OMAS SLF } \\
\hline ring & RMS & $S_{\mathrm{zz}}$ & $S_{x x}-S_{y y}$ & $\mathrm{RMS}$ & $\mathrm{S}_{\mathrm{zz}}$ & $S_{x x}-S_{y y}$ & $\mathrm{RMS}$ & $S_{\mathrm{zz}}$ & $S_{x x}-S_{y \nu}$ \\
\hline 1 & 27 & 0.51 & 0.03 & 27 & 0.40 & 0.02 & 29 & 0.50 & 0.05 \\
2 & 36 & 0.52 & 0.03 & 41 & 0.42 & 0.03 & 27 & 0.50 & 0.05 \\
\hline
\end{tabular}

Table 7.4 $\mathrm{C}-\mathrm{H}$ order parameters of the $5 \mathrm{CB}$ phenylene rings. They are obtained from the static and OMAS PDLF experiments described here, and compared with SLF results from the literature. The RMS values are in units of $\mathrm{Hz}$. The ring carrying the aliphatic chain is indicated as ring 1 and the one bearing the $\mathrm{CN}$ group as ring 2 .

We find the order parameter representing the biaxiality, $S_{x x}-S_{y y}$ to be $\sim 0.03$. A similar value was proposed before based on ${ }^{1} \mathrm{H}$ multiple-quantum NMR, ${ }^{19}$ for the case where the geometric difference between the two rings would make them distinguishable ( $D_{2}$ symmetry). A value of 0.069 was also obtained using proton dipolar couplings. ${ }^{17}$ However, it is not directly comparable to our results, since vibrational corrections, which should increase the order parameter, are not included here, in contrast to the previous calculation. We note that a sligthly higher value for the unadjusted biaxiality $\left(S_{x x}-S_{y y}=\right.$ 0.05 ) was found based on SLF-derived C-H dipolar couplings. ${ }^{10}$

A simple analysis of the type described for the aromatic $\mathrm{C}-\mathrm{H}$ couplings is not adequate for describing the dynamics of the aliphatic chain. However, we note that the 
aliphatic $\mathrm{C}-\mathrm{H}$ couplings measured here follow the same trend along the chain as observed by previous experiments. ${ }^{10}$

\subsection{Experimental Precision and Accuracy}

As discussed before, the traditional SLF experiment measures dipolar couplings through the multiplets generated by a $\left\{\mathrm{C}, \mathrm{H}_{\mathrm{n}}\right\}$ coupled spin system. Such multiplets consist of combination lines which arise from the sums and differences of various dipolar couplings. Therefore, the precision of a SLF spectrum is limited by the possibility of distinguishing the overlapping signals in the multiplet, given the maximum resolution attained with the multiple-pulse sequence and the experimental signal-to-noise ratio. The accuracy is limited by the degree of uniformity in the frequency scaling factor of the miltiple-pulse decoupling sequence throughout the spectrum. Certain multiple-pulse sequences are less sensitive than others to resonance offsets and variations in the coupling strengths ${ }^{20}$. However, even for the most favorable decoupling sequence, there is probably a variation of more than $1 \%$ in the scaling factor. Therefore, the accuracy and the precision of a SLF spectrum are of about the same magnitude.

In a PDLF experiment, the variation of the multiple-pulse scaling factor is the largest source of systematic error. The precision of the measureament is intrinsically very high, since a coupling is obtained as the simple difference between two peak maxima. This results in an uncertainty of potentially less than $0.1 \%$. However, this uncertainty cannot be taken as the accuracy of the measurement, which is at least one order of magnitude larger. The accuracy of the PDLF experiment can be evaluated by exploiting the dependence of dipolar couplings on the rotor orientation in an OMAS experiment. However, due to the particular geometry of the rf coil in the probehead used for our OMAS experiments, the rf field strength is modulated by the spinning angle in the same way. Thus the ratio of the dipolar coupling to the rf field is approximately constant over the range of angles used $\left(42^{\circ}-52^{\circ}\right)$. Consequently, the performance of the sequence as a function of the resonance 
offset may change as the spinning angle is varied. PDLF experiments performed at different angles for a given heteronuclear coupling would then experience a difference in the scaling factor of the same order for the range of frequencies observed. Therefore, the dipolar coupling values obtained from linear regression of a set of OMAS spectra should be sensitive to this source of systematic error. In other words, the error margin represents the accuracy of the PDLF measurements. The $\mathrm{C}-\mathrm{H}$ dipolar coupling strengths listed in Table 7.2 have an uncertainty of about $4-5 \%$.

\subsection{Summary}

A class of NMR techniques that allows the measurement and assignment of both short-range and long-range $\mathrm{C}-\mathrm{H}$ dipolar couplings in nematic liquid crystals has been demonstrated. The techniques apply to liquid crystals that can be oriented mechanically by fast spinning of the sample. The measurement benefits from the high resolution achievable by the PDLF technique, which can incorporate polarization transfer over large distances to detect long-range couplings. $A{ }^{13} \mathrm{C} /{ }^{1} \mathrm{H}$ chemical shift correlation experiment helps to assign the coupling partners in long-range dipolar couplings. Simultaneous measurement and assignment of the long-range couplings is achieved by, a novel reduced-3D experiment, in which the evolution periods for the $\mathrm{C}-\mathrm{H}$ couplings and ${ }^{1} \mathrm{H}$ chemical shifts are incremented separately but synchronously. This makes it possible to combine and scale two interactions in one frequency dimension, thereby facilitating the resonance assignment and enhancing the spectral resolution. The short-range $\mathrm{C}-\mathrm{H}$ dipolar couplings are determined with high precision by examining the spinning-angle dependence of the $\mathrm{C}-\mathrm{H}$ splittings, from PDLF spectra obtained with different OMAS spinning angles as well as under the static condition. From these short-range dipolar couplings, the order parameters of the phenylene rings are calculated, providing dynamic structural information about this anisotropically-mobile molecule. The PDLF-based techniques shown here constitute complementary methodologies to the traditional SLF techniques. They are expected to be 
useful in the investigation of the structure and order of many complex liquid-crystalline systems.

\section{References}

(1) Chandrasekhar, S. Liquid Crystals; Cambridge University Press, Cambridge, 1992.

(2) Nuclear Magnetic Resonance of Liquid Crystals; Emsley, J. W., Ed.; D. Reidel Publishing: Dordrecht, 1985.

(3) Photinos, D. J.; Samulski, E. T.; Toriumi, H. J. Phys. Chem. 1990, 94, 4688.

(4) NMR Spectroscopy using Liquid Crystal Solvents; Emsley, J. W.; Lindon, J. C., Ed.; Pergamon Press: Aylesbury, 1975.

(5) Ter Beek, L. C.; Zimmerman, D. S.; Burnell, E. E. Mol. Phys. 1993, 80, 177.

(6) van der Est , A. J.; Kok, M. Y.; Burnell, E. E. Mol. Phys. 1987, 60, 397.

(7) Hester, R. K.; Ackermann, J. L.; Neff, B. L.; Waugh, J. S. Phys. Rev. Lett. 1976, 36, 1081 .

(8) Opella, S. J.; Waugh, J. S. J. Chem. Phys. 1977, 66, 4919.

(9) Fung, B. M.; Afzal, J. J. Am. Chem. Soc. 1986, 108, 1107.

(10) Fung, B. M.; Afzal, J.; Foss, T. L.; Chau, M. J. Chem. Phys. 1986, 85, 4808.

(11) Courtieu, J.; Alderman, D. W.; Grant, D. M.; Bayles, J. P. J. Chem. Phys. 1982, $77,723$.

(12) Courtieu, J.; Bayle, J. P.; Fung, B. M. Prog. in NMR Spect. 1994, 26, 141.

(13) Weitekamp, D. P.; Garbow, J. R.; Pines, A. J. Chem. Phys. 1982, 77, 2870.

(14) Caravatti, P.; Bodenhausen, G.; Ernst, R. R. Chem. Phys. Lett. 1982, 89, 363.

(15) Nakai, T.; Terao, T. Magn. Reson. Chem. 1992, 30, 42.

(16) Schmidt-Rohr, K.; Nanz, D.; Emsley, L.; Pines, A. J. Phys. Chem 1994, 98, 6668 . 
(17) Emsley, J. W.; Luckhurst, G. R.; Stockley, C. P. Mol. Phys. 1981, 44, 565.

(18) Emsley, J. W.; Luckhurst, G. R.; Gray, G. W.; Mosley, A. Mol. Phys. 1978, $35,1499$.

(19) Sinton, S. W.; Zax, D. B.; Murdoch, J. B.; Pines, A. Mol. Phys. 1984, 53, 333.

(20) Guo, W.; Fung, B. M. J. Chem. Phys. 1991, 95, 3917.

(21) Caravatti, P.; Braunschweiler, L.; Ernst, R. R. Chem. Phys. Lett. 1983, 100, 305.

(22) Roberts, J. E.; Vega, S.; Griffin, R. G. J. Am. Chem. Soc. 1984, 106, 2506.

(23) Eastman, M. A.; Grandinetti, P. J.; Lee, Y. K.; Pines, A. J. Magn. Res $\_n$ 1992, $98,333$.

(24) Mansfield, P. J. Phys. Chem. 1971, 4, 1444.

(25) Rhim, W.-K.; Elleman, D. D.; Vaughan, R. W. J. Chem. Phys. 1973, 59, 1740.

(26) States, D. J.; Haberkorn, R. A.; Ruben, D. J. J. Magn. Reson. 1982, 48, 286.

(27) Caravatti, P.; Neuenschwander, P.; Ernst, R. R. Macromolecules 1985, 18, 119.

(28) Bodenhausen, G.; Ernst, R. R. J. Magn. Reson. 1981, 45, 367.

(29) Bodenhausen, G.; Ernst, R. R. J. Am. Chem. Soc. 1982, 104, 1304.

(30) Hong, M.; Schmidt-Rohr, K.; Pines, A. J. Am. Chem. Soc. 1995b, 117, 3310.

(31) Jameson, C. J. In Multinuclear NMR; J. Mason, Ed.; Plenum Press: New York, 1987; pp 89.

(32) Zannoni, C. Nuclear Magnetic Resonance of Liquid Crystals; D. Reidel Publishing Company, Dordrecht, 1985. 
APPENDICES

\section{A 4-spin density matrix evolution}

In chapter 5, the evolution of the density operator under the DISTINCT pulse sequence was described. The crucial step in this pulse sequence is to convert the unobservable sine-modulated dipolar coherence into observable magnetization through isotropic heteronuclear $J$ coupling. Here, we describe the detailed time evolution of a 4spin system, a $\mathrm{CH}_{3}$ group, under such a coherence transfer. This complements the 3 -spin case $\left(\mathrm{CH}_{2}\right)$ presented in chapter 5 .

The density operator at the beginning of the coherence transfer period is

$$
\rho(0)=2 S_{x} I_{z} \sin \left(\omega_{C H} t_{1}\right)=2 S_{x}\left(I_{z}^{a}+I_{z}^{b}+I_{z}^{c}\right) \sin \left(\omega_{C H} t_{1}\right) .
$$

where $a, b$, and $c$ refer to the three protons in the $\mathrm{CH}_{3}$ group. This density operator evolves under the $J$ coupling Hamiltonian,

$$
H_{J}=2 \omega_{j}^{a} \cdot S_{z} I_{z}^{a}+2 \omega_{j}^{b} \cdot S_{z} I_{z}^{b}+2 \omega_{j}^{c} \cdot S_{z} I_{z}^{c}
$$

Since different spin operators $I_{z}^{i}(i=a, b, c)$ commute, the antiphase operators in (A.1) evolve under the three $J$ couplings sequentially,

$$
\rho(t)=e^{-i H_{c} \tau} e^{-i H_{b} \tau} e^{-i H_{a} \tau} \cdot \rho(0) \cdot e^{i H_{a} \tau} e^{i H_{b} \tau} e^{i H_{c} \tau} .
$$

Consider the $a$-spin operator $\rho^{a}(0)=2 S_{x} I_{z}^{a} \sin \left(\omega_{C H} t_{1}\right)$. It evolves under the $S_{z} I_{z}^{a}$ coupling in (A.2) according to 


$$
S_{x} I_{z}^{a} \stackrel{H_{a}}{\longrightarrow} S_{x} I_{z}^{a} \cos \left(\omega_{j}^{a} \tau\right)+2 \cdot \frac{1}{4} \cdot S_{y} \sin \left(\omega_{j}^{a} \tau\right)
$$

The observable magnetization $S_{y}$ is unaffected by subsequent evolutions under the two other $J$ couplings, except that it acquires a modulation factor $\cos \left(\omega_{j}^{b} \tau\right) \cos \left(\omega_{j}^{c} \tau\right)$. Grouping all the factors for $\rho^{a}(0)$, one obtains the final $a$-spin magnetization as

$$
\rho^{a}(t)=2 \cdot 2 \cdot \frac{1}{4} S_{y} \sin \left(\omega_{j}^{a} \tau\right) \cos \left(\omega_{j}^{b} \tau\right) \cos \left(\omega_{j}^{c} \tau\right) \cdot \sin \left(\omega_{C H} t_{1}\right)
$$

For $\rho^{b}(0)$ and $\rho^{c}(0)$, the observable magnetization is modulated similarly with appropriate permutations of (A.5) with respect to the spin index,

$$
\begin{aligned}
& \rho^{b}(t)=2 \cdot 2 \cdot \frac{1}{4} S_{y} \cos \left(\omega_{j}^{a} \tau\right) \sin \left(\omega_{j}^{b} \tau\right) \cos \left(\omega_{j}^{c} \tau\right) \cdot \sin \left(\omega_{C H} t_{1}\right), \\
& \rho^{c}(t)=2 \cdot 2 \cdot \frac{1}{4} S_{y} \cos \left(\omega_{j}^{a} \tau\right) \cos \left(\omega_{j}^{b} \tau\right) \sin \left(\omega_{j}^{c} \tau\right) \cdot \sin \left(\omega_{C H} t_{1}\right) .
\end{aligned}
$$

Therefore, the sum magnetization is

$$
\begin{aligned}
\rho(t)= & S_{y} \sin \left(\omega_{C H} t_{1}\right)\left\{\sin \left(\omega_{j}^{a} \tau\right) \cos \left(\omega_{j}^{b} \tau\right) \cos \left(\omega_{j}^{c} \tau\right)\right. \\
& \left.+\cos \left(\omega_{j}^{a} \tau\right) \sin \left(\omega_{j}^{b} \tau\right) \cos \left(\omega_{j}^{c} \tau\right)+\cos \left(\omega_{j}^{a} \tau\right) \cos \left(\omega_{j}^{b} \tau\right) \sin \left(\omega_{j}^{c} \tau\right)\right\}
\end{aligned}
$$

The terms in the big bracket in (A.8) can be simplified to

$$
\sin \left(\omega_{j}^{a}+\omega_{j}^{b}+\omega_{j}^{c}\right) \tau+\sin \omega_{j}^{a} \tau \cdot \sin \omega_{j}^{b} \tau \cdot \sin \omega_{j}^{c} \tau
$$

Since the one-bond $\mathrm{C}-\mathrm{H} J$ coupling is the same for all protons in a $\mathrm{CH}_{3}$ group, the above expression can be simplified further to obtain the following density operator, 


$$
\rho\left(t_{1}, \tau\right)=S_{y} \sin \left(\omega_{C H} t_{1}\right) \cdot\left[\sin \left(3 \omega_{j} \tau\right)+\sin ^{3}\left(\omega_{j} \tau\right)\right]
$$

This magnetization encodes the dipolar couplings through $\sin \left(\omega_{\mathrm{CH}} t_{1}\right)$, and is detected in the acquisition period. With the $J$ coupling period $\tau$ fixed, the frequency spectrum is the Fourier transform of the signal (A.10) with respect to $t_{1}$. This would give rise to antisymmetric and absorptive lineshapes in the $\omega_{1}$ dimension of the $2 \mathrm{D}$ spectrum, with two lines occurring at the dipolar frequencies $\pm \omega_{\mathrm{CH}}$.

\section{B Equivalence of Motional Averaging in Fast Motional Limit}

If a molecular segment undergoes several motions that are all fast on the NMR time scales, then the averaged NMR frequencies are independent of the sequence in which the averaging is calculated. This can be demonstrated for a phenyl ring that undergo both fast rotations around an axis in the ring plane and $180^{\circ}$ flips around its $C_{2}$ axis. In the following, we prove that the effects of the two motions on second-rank tensor interactions such as quadrupolar coupling are not influenced by the sequence of averaging.

(a)

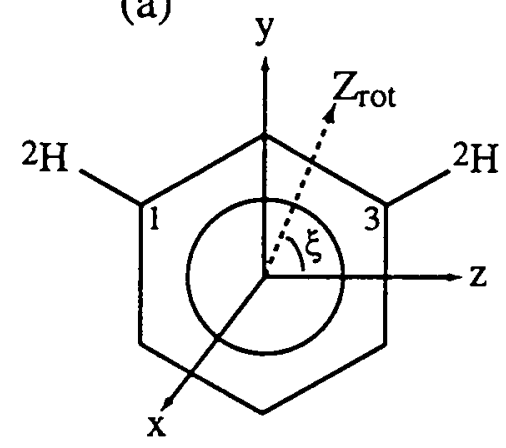

(b)

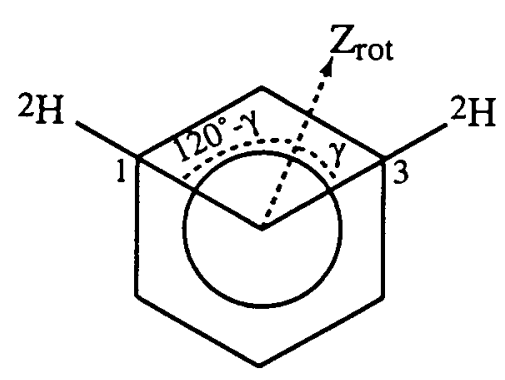

Figure B.1 Equivalence of motional averaging of a phenyl ring. (a) The ${ }^{2} \mathrm{H}$ quadrupolar couplings are averaged first by $180^{\circ}$ flips, then by rotations. (b) The ${ }^{2} \mathrm{H}$ splittings are averaged by rotations first and then by the two-site exchange. 
Case 1: The $\mathrm{C} 1$ and $\mathrm{C} 3$ ring carbons undergo fast exchange by $180^{\circ}$ flips (Figure B.1a). This averages the quadrupolar interaction along the two $\mathrm{C}-2 \mathrm{H}$ bonds. The resulting average $y$-axis is along the flip axis and the $z$-axis is in the plane of the ring and perpendicular to $y$. The averaged quadrupolar coupling is calculated as

$$
\bar{\delta}=\frac{1}{2} \delta_{0}\left(3 \cos ^{2} \beta-1-\eta_{0} \sin ^{2} \beta \cos 2 \alpha\right),
$$

where $\delta_{0}$ is the unaveraged quadrupolar coupling and $\beta$ is the angle between each $\mathrm{C}-2 \mathrm{H}$ bond and the averaged $z$-axis. Since $\eta_{0}=0$ for the original quadrupolar interaction,

$$
\bar{\delta}=\frac{1}{2} \delta_{0}\left(3 \cos ^{2} 30^{\circ}-1\right)=\frac{5}{8} \delta_{0}
$$

For the averaged quadrupolar tensor, the asymmetry parameter is not necessarily zero. In fact, it has been calculated to be 0.6 for this case.

This once-averaged NMR frequency $\bar{\delta}$ is averaged again by the phenylene ring rotation around an axis that lies in the ring plane and forms an angle $\xi$ with the $z$-axis of the averaged tensor. The final quadrupolar coupling is

$$
\begin{aligned}
\overline{\bar{\delta}} & =\frac{1}{2} \bar{\delta}\left(3 \cos ^{2} \xi-1-\bar{\eta} \sin ^{2} \xi \cos \left(2 \cdot 90^{\circ}\right)\right) \\
& =\frac{1}{2} \frac{5}{8} \delta_{0}\left(3 \cos ^{2} \xi-1+0.6 \sin ^{2} \xi\right)=\frac{5}{16} \delta_{0}\left(\frac{4}{5}+\frac{6}{5} \cos 2 \xi\right) . \\
& =\frac{1}{4} \delta_{0}\left(1+\frac{3}{2} \cos 2 \xi\right)
\end{aligned}
$$

Case 2: We consider averaging by rotation first. The $\mathrm{C} 1{ }^{2} \mathrm{H}$ and the $\mathrm{C} 3{ }^{2} \mathrm{H}$ bonds, which form angles $120^{\circ}-\gamma$ and $\gamma$ with the rotation axis, respectively (Figure B.1b), yield different averaged quadrupolar couplings,

$$
\bar{\delta}_{1}=\delta_{0} \cdot \frac{1}{2}\left(3 \cos ^{2}\left(120^{\circ}-\gamma\right)-1\right), \quad \bar{\delta}_{3}=\delta_{0} \cdot \frac{1}{2}\left(3 \cos ^{2} \gamma-1\right),
$$


The two averaged quadrupolar tensors are collinear to the common rotational axis $Z_{r o t}$. Thus they can be simply added to account for the second averaging by $180^{\circ}$ ring flips,

$$
\begin{aligned}
\overline{\bar{\delta}} & =\frac{1}{2}\left(\bar{\delta}_{1}+\bar{\delta}_{3}\right)=\frac{1}{2} \frac{\delta_{0}}{2}\left(3 \cos ^{2} \gamma+3 \cos ^{2}\left(120^{\circ}-\gamma\right)-2\right) \\
& =\frac{\delta_{0}}{4}\left(1+\frac{3}{4} \cos 2 \gamma-\frac{3 \sqrt{3}}{4} \sin 2 \gamma\right)
\end{aligned} .
$$

Since $\gamma=\xi-30^{\circ}$ (Figure B.1), the last equation can be simplified to

$$
\begin{aligned}
\overline{\bar{\delta}} & =\frac{\delta_{0}}{4}\left(1+\frac{3}{4} \cos \left(2 \xi-60^{\circ}\right)-\frac{3 \sqrt{3}}{4} \sin \left(2 \xi-60^{\circ}\right)\right) \\
& =\frac{\delta_{0}}{4}\left(1+\frac{3}{2} \cos 2 \xi\right)
\end{aligned}
$$

Comparison of equations (B.3) and (B.6) shows that the averaging of a uniaxial tensor by two fast motions is independent of the sequence of the averaging.

\section{Epsilon Motor Controller System}

\section{Angle Setting}

The commands for manually controlling motor positions and hopping the probehead to various angles are typed in the "dummy" mode of the PC. After using the "Esc" key to reach the main menu, one chooses Item 0 to enter the dummy mode, where the following commands are used in setting the angles.

- ORAI\# for going to the absolute position \#.

- ORP for reporting the current position.

- OPIZ for setting the current position as 0.

- OAM\# for increasing an angle \# from the current position.

- OIM\# for decreasing an angle \# from the current position.

The commands OAM and OIM are always used in conjunction in a DAS-type experiment that involves only a pair of angular positions. 
Item 1 allows the user to view controller information. The controller address is specified as 0 at all times. The Baud Rate is 1200, and the DIR entry is CCS1.

Item 2 is for viewing profile information. The profiles must be redefined when the user switches between a DAS-type experiment and a VACSY experiment. The commands are:

- ODE1 for defining profile 1. The computer responds " *RAI\# " if an angle \# is already stored in the address $\mathrm{DE} 1$, and *ED if there is nothing.

- ORAI\# for storing the angular position \# in the profile.

- OED to end profile editing.

Note that even if the old profile already has the correct angular position, the user still needs to type the RAI value, as long as the the definition process has already been started. Otherwise the old information will be lost. Continue defining the profiles until all angles are put in.

\section{D.I.P. switches for VACSY}

SW1: $\mathbf{R}$ L $R$ R L L L L

$1-3:$ step size $=1 / 32$ steps $; 4: R=$ optical encoder feedback is on. Optical encoder is on to replace the previous step size with a 1024-line with a multiplier of $4 ; 5-8$ : output current to the motor.

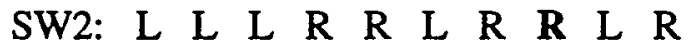

1-3: unit address; 4 : master unit; 6 : baud rate, $L=1200 ; 7$ : serial standard, RS232C; 8: profile lines, $\mathrm{R}=$ encoded for VACSY.

\section{VACSY Hopping Profile}

In profile 1, set: RAT, MW, RFU, MW, ED.

D.I.P. switches for DAS

SW1: L L $R \quad R \quad$ L L L L 1-3: step size $=1 / 16$;

SW2: L L L R R L R $\quad$ L L $\quad$ R 8: $L=$ non-encoded profile lines for hopping experiments; 9-10: $L R=$ position pulse multiplier is 4 .

\section{TTL Connections}


encoded profiles (VACSY):

B3 $\rightarrow 1$

B4 $\rightarrow 2$

B5 $\rightarrow 3$

B6 $\rightarrow 4$

B7 $\rightarrow 5$

A2 $\rightarrow 6$ non-encoded profiles (DAS and SAS):

A2 $\rightarrow$ profile trigger line 1

A3 $\rightarrow$ trigger 1; hop to first angle

B1 $\rightarrow$ trigger 2; hop to second angle

\section{Encoder Ratio}

$\frac{\text { motor step }(200) * \text { step size }(S W 1,1 \sim 3)}{\text { line per revolution encoder }(400,1024) * \text { position pulse multiplier }(S W 2,9 \sim 10)}$

Most encoders in the lab have 1024 lines per revolution. This corresponds to an encoder ratio of 6400 , if the step size is $1 / 32$.

Reference Values for Motor Controller Parameters

no significant loss of angle ( $<40 \mathrm{~ms})$ : $\quad$ safe $(<60 \mathrm{~ms})$ :

AC: $1,000,000 \quad$ AC: 50,000

SS: $3,000 \quad$ SS: 2,000

SP: $10,000 \quad$ SP: 2,000

\section{Debugging}

For continuous rotation, set 0ER4096 as the encoder ratio. The normal default is 0 ER6400.

If the motor rotates all the way to the limit, turn off the computer and then the motor controller. Turn the controller on again, make sure the fault light is off, and then reboot the computer.

For position correction, type 0PC1.

To reactivate the computer when it is stuck, type OWB. If the $\mathrm{PC}$ and the motor are still stuck, restart the PC. Turn off and on the motor, then reboot the computer. The rotor position should be in the memory after the system is back on.

\section{Computer Programs}


FORTRAN language is used in the program that calculates model-independent maximum orientation probabilities (D.1). Both FORTRAN and MATLAB languages are used for calculating and plotting, on a unit sphere, Gaussian distributions that have the required principal values of a segmental order tensor (D.2). Various approximations to the original segmental orientation distribution are also simulated.

\section{Model-Independent Orientation Probabilities from a Negative Order Parameter}

c PROGRAM maxorient.f

c calculate the upper limit probability below a certain angle, assuming a fixed bond order parameter

c $\mathrm{SCH}$, and a double Gaussian distribution, in which the second Gaussian is at $90^{\circ}$.

parameter (maxnumbroad $=5$ )

REAL peak (maxnumbroad, 0:360, 0:360)

REAL pi, SCH

REAL delm(20), $\operatorname{scx}(20,0: 400)$

REAL xdeg,xg,asc,ac

REAL P2(0:400), jtem, item

REAL Int90,Intx,P2ba90(20), P2bax, Intp $(20,1000)$

REAL theta, gauff,tvar, itv,delta(20)

REAL gauf(20, 0:2000), c(20, 0:1000), $\operatorname{Imax}(20,0: 300)$

REAL optxvar(20, 0:300),distf1(20, 0:1000), distf2(20,0:1000)

INTEGER n,nd,ng,isc,jsc,ic,jc,k,kx,j,ith,it,iz,jz,t,i,kidx

write $\left(6,{ }^{*}\right)$ 'This program calculates curves which represent limits to the

write $\left(6,{ }^{*}\right)$ 'integral orientational probability for a given order parameter'

write $(6, *)$ 'assuming a minimal broadening.'

write $(6, *)$ 'The smaller the broadening, the more general the result.'

write $\left(6,{ }^{*}\right)$ 'The maximum \# of broadening values is currently'

write $(6, *)$ maxnumbroad

write $(6, *)$ 'number of broadenings -->'

$\operatorname{read}(5, *)$ dnb

do 5 , ibroad $=1$, dnb

write $(6, *)$ 'Gaussian broadening -->'

$\operatorname{read}(5, *)$ delta(ibroad)

5

continue

write $(*, *)$ 'bond order parameter (e.g.-0.2) -->'

$\operatorname{read}(*, *)$ SCH

$\mathrm{pi}=3.1415927$

rads $=p i / 720$.

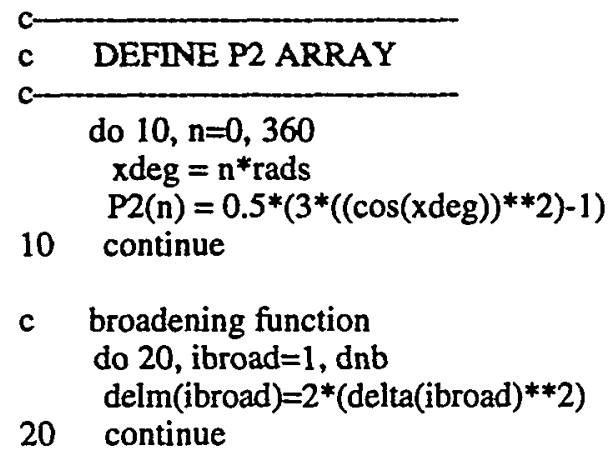




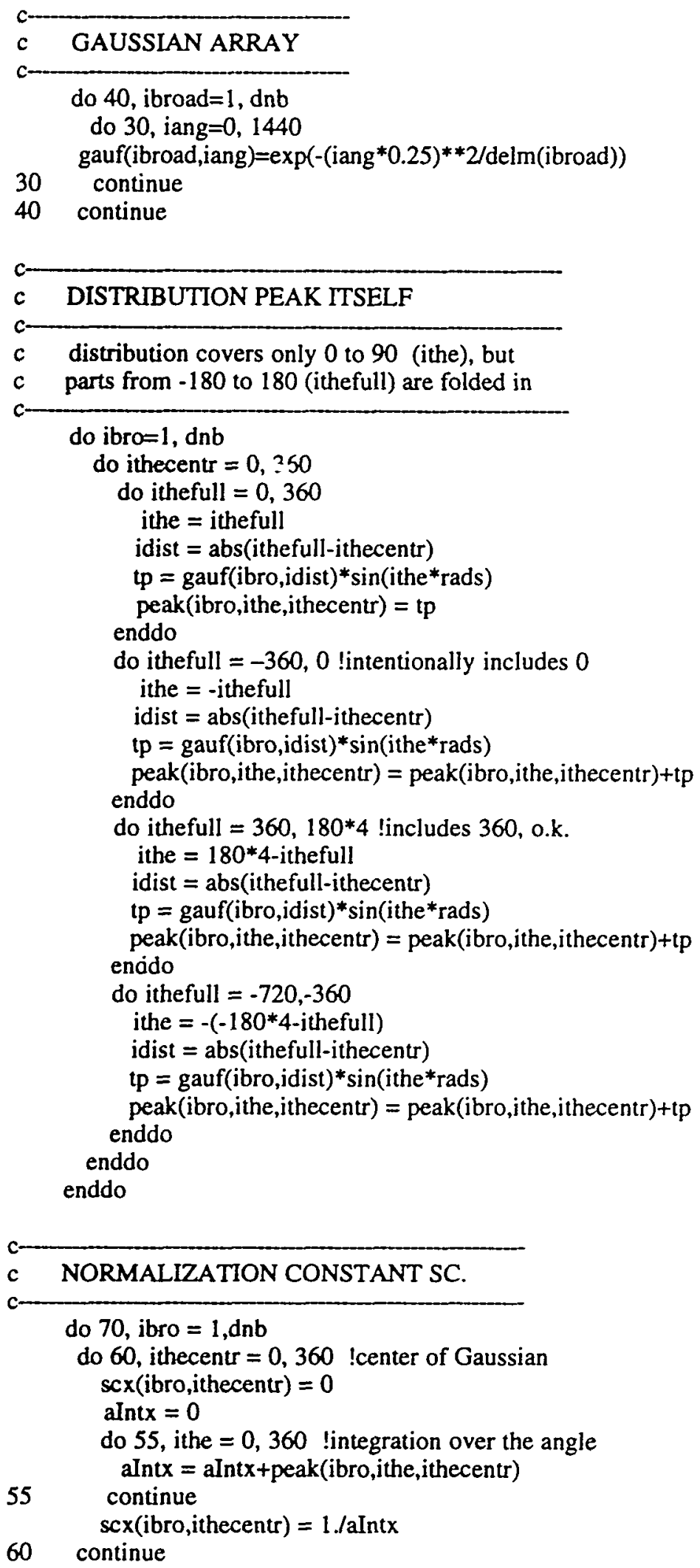




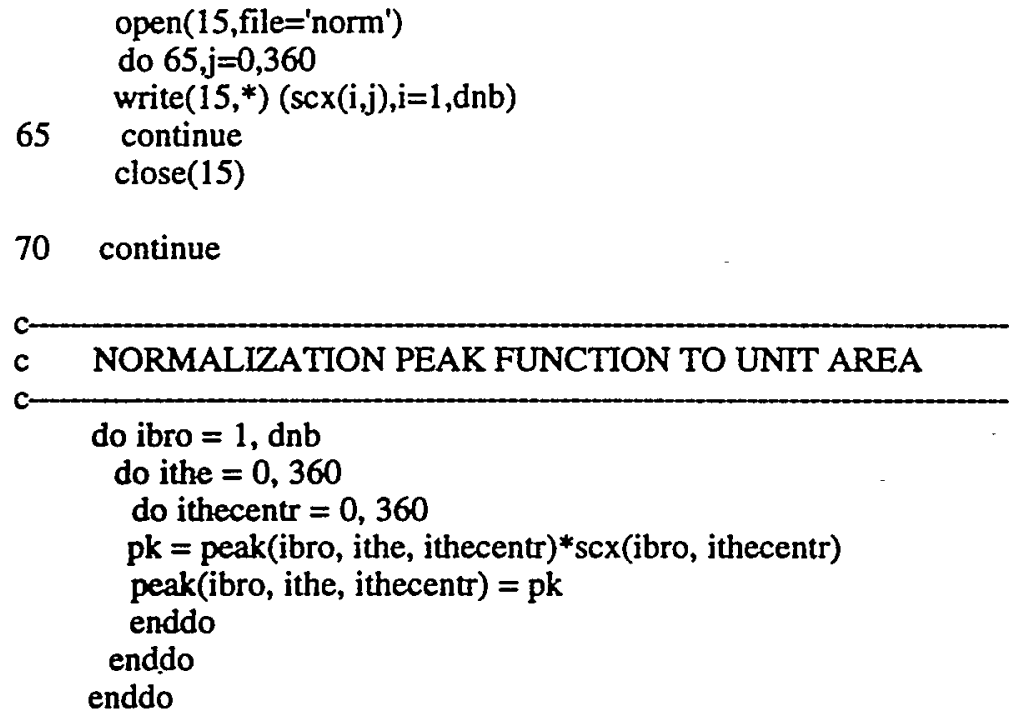

c FOR EACH BROADENING, PLOT ONE IMAX(THETA) CURVE. do 200 , ibro $=1$, dnb

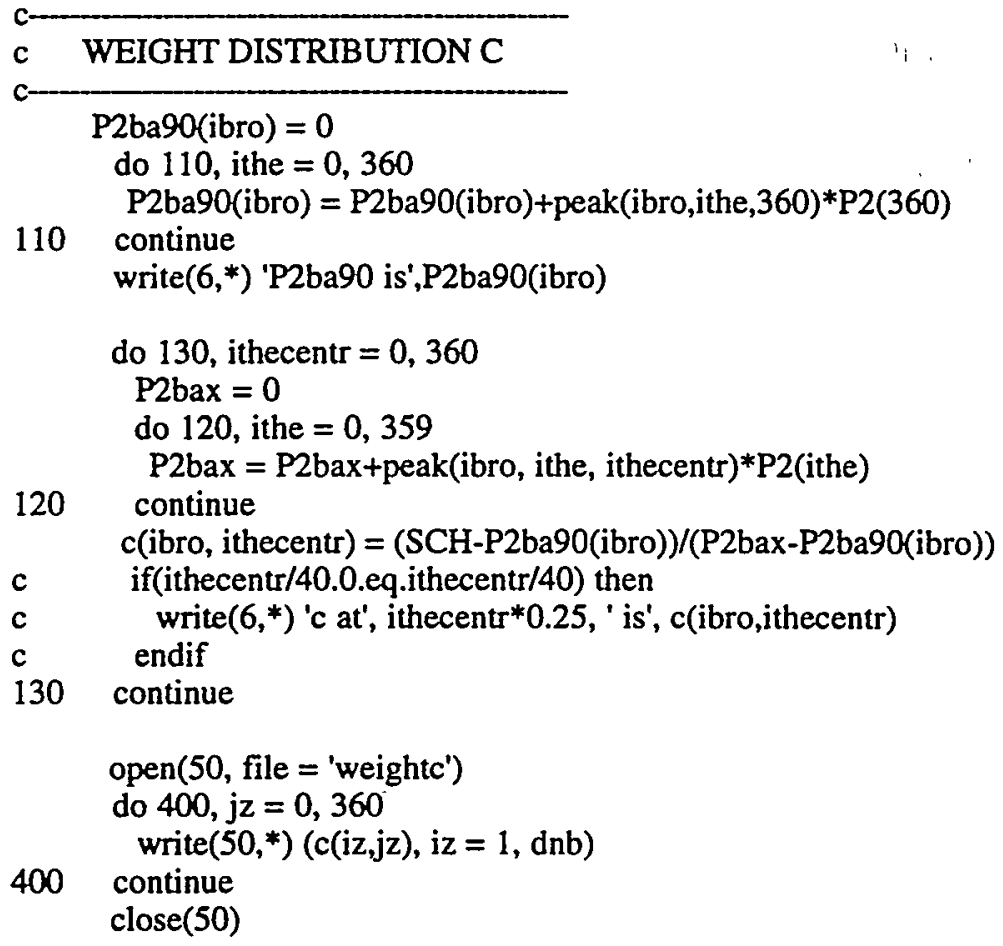

c I AS A FUNCTION OF ithecentr

do 160 , ithecentr $=0,360$ ! shift center, looking for max I(the) $\mathrm{aI}=0$ 


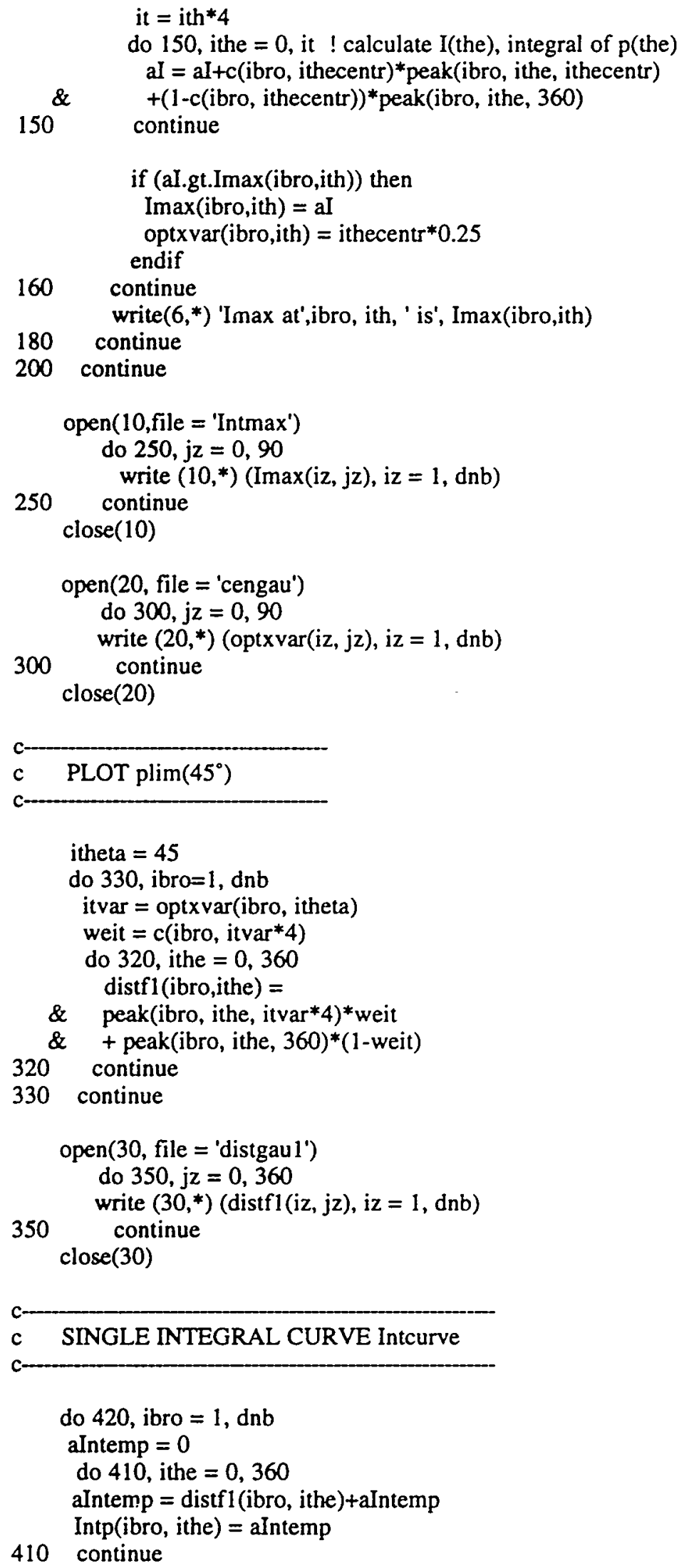


open (60, file $=$ 'Intcurve')

do $450, \mathrm{jz}=0,360$

450

write $(60, *)(\operatorname{Intp}(\mathrm{iz}, \mathrm{jz}), \mathrm{iz}=1, \mathrm{dnb})$

END

\section{Orientation Distributions from Segmental Order Tensors}

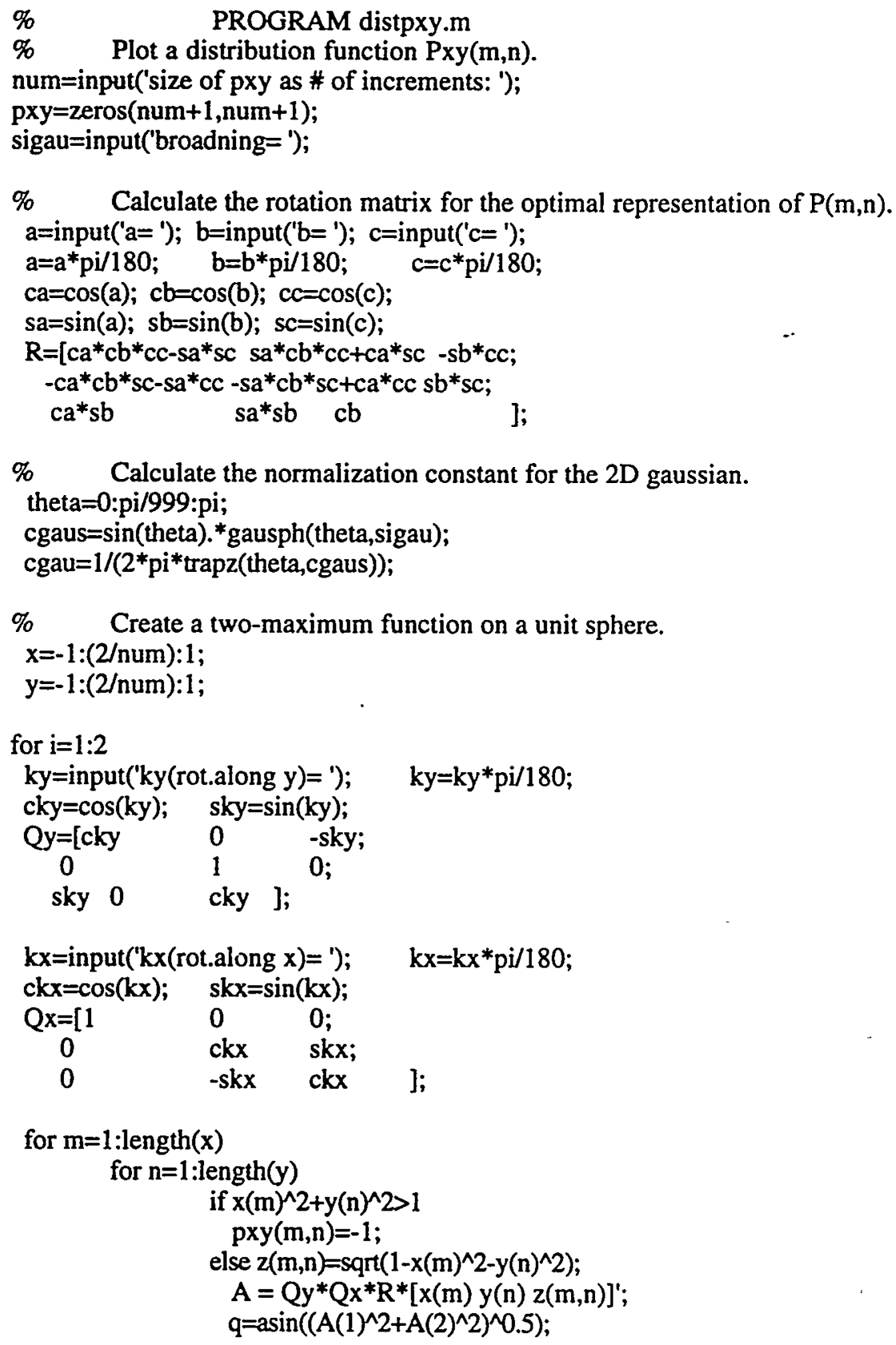

\% Calculate the rotation matrix for the optimal representation of $\mathrm{P}(\mathrm{m}, \mathrm{n})$. $a=$ input(' $a=$ '); b=input('b= '); c=input('c= '); 


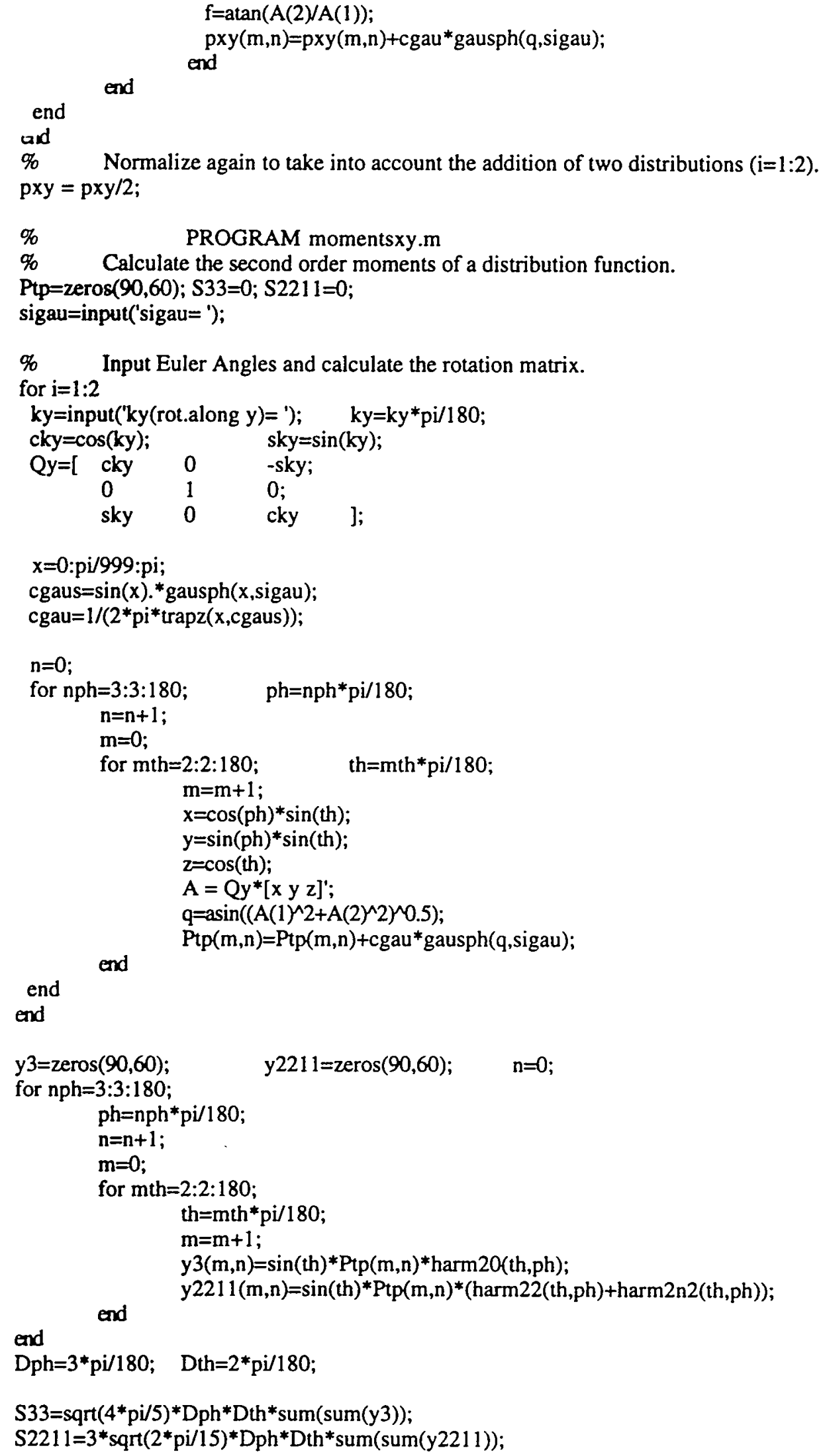




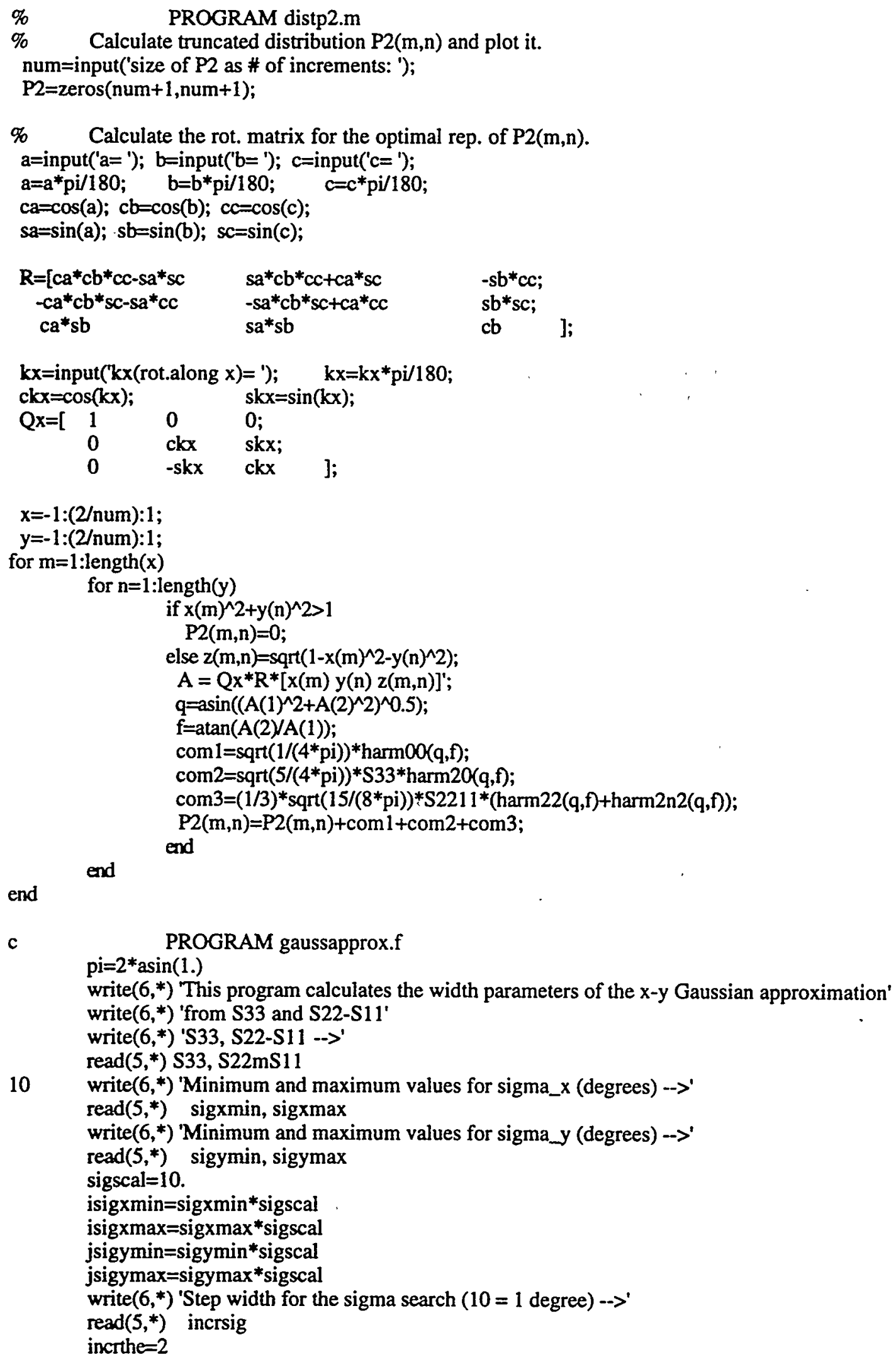


incrphi $=2$

erromin $=999$

c VARY sigma_x and sigma $y$

do isig $\mathrm{x}=$ isigxmin, isigxmax,incrsig

sig $x=i s i g x /$ sigscal ${ }^{*} \mathrm{pi} / 180$.

do jsigy=jsigymin,jsigymax,incrsig

sigy=jsigy/sigscal* ${ }^{*} \mathrm{pi} / 180$

c CALCULATE ONLY IF PRECISION IS NOT GOOD ENOUGH if(erromin.gt.0.0001) then

c NORMALIZATION

anorm $=0$.

do ithe $=1,180$, incrthe

the $=$ ithe

do iphi $=1,180$,incrphi

phi=iphi

$\mathrm{x}=\operatorname{cosd}(\mathrm{phi}) * \operatorname{sind}($ the $)$

$y=\operatorname{sind}(\mathrm{phi}) * \operatorname{sind}($ the $)$

anorm $=$ anorm $+\operatorname{sind}($ the $) * \exp \left(-0.5^{*}\left((\mathrm{x} / \mathrm{sigx})^{* *} 2+(\mathrm{y} / \mathrm{sigy})^{* * 2}\right)\right)$

enddo

enddo

cgau $=1$ /anorm

c

write $\left(6,{ }^{*}\right)^{\prime} \operatorname{cgau}={ }^{\prime}, \mathrm{cgau} /(2 . * 3.141)$

c INTEGRATE TO OBTAIN CALCULATED MOMENTS

S33calc $=0$.

S2211 calc $=0$.

do ithe $=1,180$, incrthe

the $=$ ithe

do iphi $=1,180$,incrphi

phi=iphi

$x=\operatorname{cosd}(p h i) * \operatorname{sind}($ the $)$

$y=\operatorname{sind}(p h i) * \operatorname{sind}($ the $)$

S33calc $=S 33$ calc + cgau $*$ sind $($ the $) * \exp \left(-0.5^{*}\left((\mathrm{x} / \mathrm{sigx})^{* *} 2+(\mathrm{y} / \mathrm{sigy})^{* *} 2\right)\right)$

\&

$* 0.5 *(3 *(\cos d($ the $)) * * 2-1)$

S2211 calc $=S 2211$ calc + cgau* $* \exp \left(-0.5 *\left((x / \text { sigx })^{* *} 2+(y / \text { sigy })^{* * 2}\right)\right)$

\& $\quad * 1.5 *(\operatorname{sind}(\text { the }))^{* * 3 *} \cos d(2 *$ phi $)$

enddo

enddo

c COMPARE CALCULATED MOMENTS WITH GIVEN MOMENTS

write $(6, *)^{*}$ S33,S2211',S33calc,S2211calc

erro $=\operatorname{sqrt}((\mathrm{S} 33-\mathrm{S} 33 \mathrm{calc}) * * 2+(\mathrm{S} 22 \mathrm{mS} 11-\mathrm{S} 2211 \mathrm{calc}) * * 2)$

if(erro.le.erromin) then

erromin=erro

sigxbest $=s i g x * 180 . / p i$

sigybest $=s i g y * 180 . / \mathrm{pi}$

dminS33=abs(S33-S33calc)

dminS221 1=abs(S22mS11-S2211 calc)

endif

endif ! dmin

enddo ! isigy

enddo !isigx

write $\left(6,{ }^{*}\right)^{\prime}$ deviation in S33 and S22-S11',dminS33,dminS2211

write $\left(6,{ }^{*}\right)$ 'sigma_ $x=$ ',sigxbest

write $\left(6,{ }^{*}\right)$ 'sigma_y $=$ ',sigybest 
write $\left(6,{ }^{*}\right)^{\prime}$ 'do it again $(0 / / 1)-->$

$\operatorname{read}(5, *)$ iagain

if(iagain.eq.1) goto 10

stop

end

PROGRAM maxS33.f

write $\left(6,{ }^{*}\right)$ 'find averaged tensor values for sum of two tensors with shared 33-11 plane'

write $(6, *)$ 'first tensor: S33, S22-S11, prob. -->'

$\operatorname{read}(5, *) \quad S 33, S 22 \mathrm{mS} 11, \mathrm{p}$

$\mathrm{S} 11=-0.5^{*}(\mathrm{~S} 33+\mathrm{S} 22 \mathrm{mS} 11)$

$\mathrm{S} 22=-\mathrm{S} 33-\mathrm{S} 11$

write $(6, *)$ 'S11=', S11

write $(6, *)$ 'second tensor: S33, S22-S11, rotated by -->'

$\operatorname{read}(5, *) S p 33, S p 22 \mathrm{mS11}, \mathrm{psi0}$

$\mathrm{Sp} 11=-0.5 *(\mathrm{Sp} 33+\mathrm{Sp} 22 \mathrm{mS} 11)$

$\mathrm{Sp} 22=-\mathrm{Sp} 33-\mathrm{Sp} 11$

write $(6, *)$ 'Sp11= ', Sp11

psincr $=-$ psio/ 10000 .

$\mathrm{psi}=\mathrm{psi0}$

sumrot $=-999$

10 sumrotprev $=$ sumrot

$\mathrm{s} 2=(\operatorname{sind}(\mathrm{psi}))^{* * 2}$

$\mathrm{s} 2 \mathrm{p}=(\operatorname{sind}(\mathrm{psi}-\mathrm{psi} 0))^{* * 2}$

sumrot $=\mathrm{p}^{*}(\mathrm{~S} 11 * \mathrm{~s} 2+\mathrm{S} 33 *(1-\mathrm{s} 2))+(1-\mathrm{p}) *(\mathrm{Sp} 11 * \mathrm{~s} 2 \mathrm{p}+\mathrm{Sp} 33 *(1-\mathrm{s} 2 \mathrm{p}))$

if(sumrot.gt.sumrotprev) then

psi $=$ psi + psincr

goto 10

endif

psibest $1=$ psi-psincr

S33av = sumrot

psincr $=-$ psincr

psi $=0$

sumrot $=-999$

20 sumrotprev=sumrot

$\mathrm{s} 2=(\operatorname{sind}(\mathrm{psi})) * * 2$

$\mathrm{s} 2 \mathrm{p}=(\operatorname{sind}(\mathrm{psi}-\mathrm{psi} 0))^{* * 2}$

sumrot $=p^{*}(S 11 * s 2+S 33 *(1-s 2))+(1-p) *(S p 11 * s 2 p+S p 33 *(1-s 2 p))$

if(sumrot.gt.sumrotprev) then

psi=psitpsincr

goto 20

endif

psibest $2=$ psi-psincr

psibest $=0.5^{*}$ (psibest $1+$ psibest 2 )

S33av $=0.5^{*}(\mathrm{~S} 33 \mathrm{av}+\mathrm{sumrot})$

S22av $=$ p*S22+(1-p)*Sp22

$\mathrm{S} 1 \mathrm{av}=-\mathrm{S} 33 \mathrm{av}-\mathrm{S} 22 \mathrm{av}$

S22mS11av = S22av-S11av

write $\left(6,{ }^{*}\right)$ 'S33av, S22av-S11av', S33av, S22mS11av

write $(6, *)$ 'psi, uncertainty', psibest, psincr

stop 
end

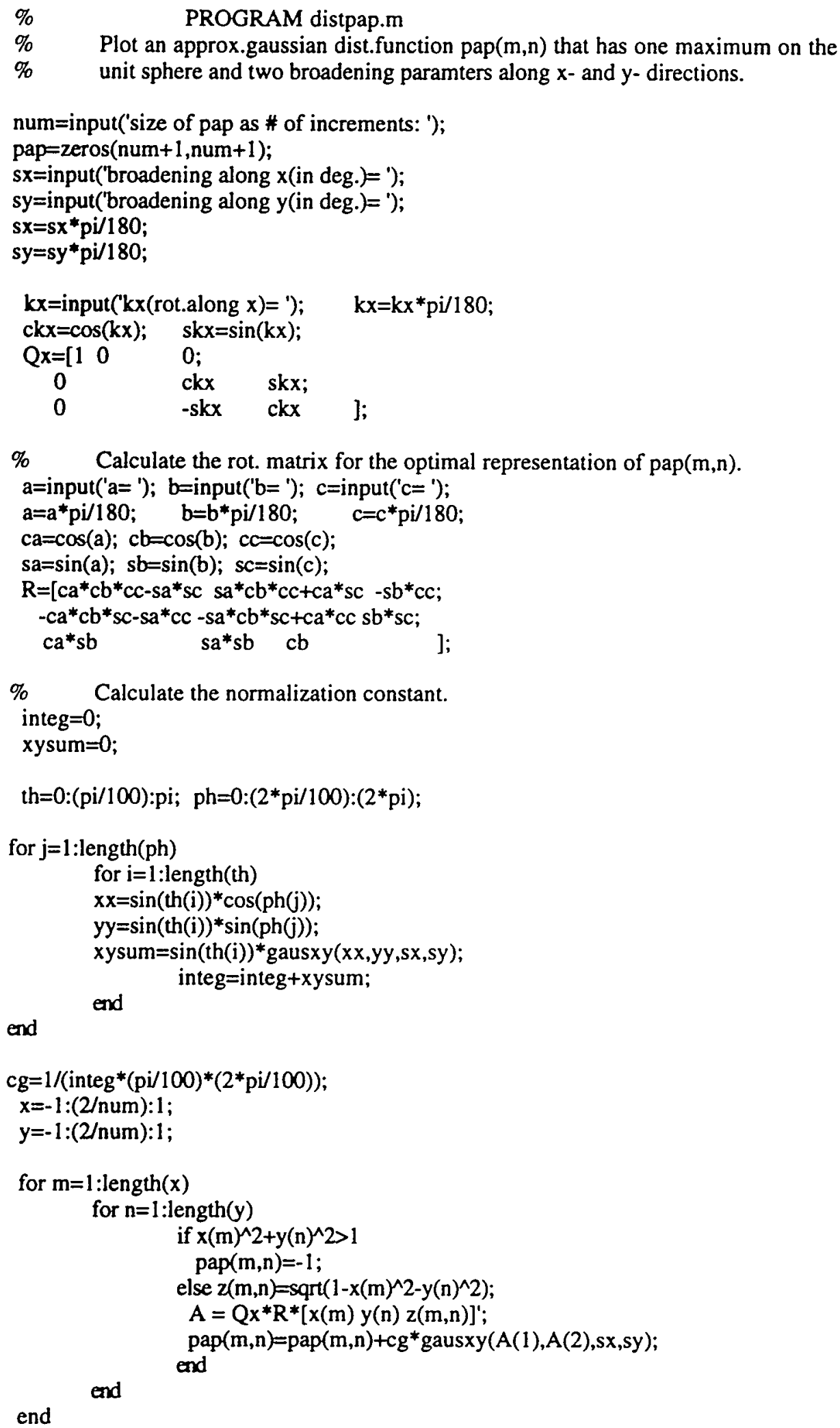

